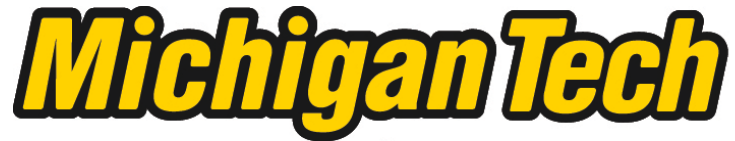 \\ Michigan Technological University Create the Future Digital Commons @ Michigan Tech
}

Dissertations, Master's Theses and Master's Reports - Open

Dissertations, Master's Theses and Master's

Reports

2014

NON-CHROMATOGRAPHIC PURIFICATION OF SYNTHETIC BIOOLIGOMERS

Durga Prasad Pokharel

Michigan Technological University

Follow this and additional works at: https://digitalcommons.mtu.edu/etds

Part of the Chemistry Commons

Copyright 2014 Durga Prasad Pokharel

\section{Recommended Citation}

Pokharel, Durga Prasad, "NON-CHROMATOGRAPHIC PURIFICATION OF SYNTHETIC BIO-OLIGOMERS", Dissertation, Michigan Technological University, 2014.

https://doi.org/10.37099/mtu.dc.etds/865

Follow this and additional works at: https://digitalcommons.mtu.edu/etds

Part of the Chemistry Commons 


\title{
NON-CHROMATOGRAPHIC PURIFICATION OF SYNTHETIC BIO-OLIGOMERS
}

\author{
By \\ Durga Prasad Pokharel
}

A DISSERTATION

Submitted in partial fulfillment of the requirements for the degree of

DOCTOR OF PHILOSOPHY

In Chemistry

MICHIGAN TECHNOLOGICAL UNIVERSITY

2014

(C) 2014 Durga Prasad Pokharel 
This dissertation has been approved in partial fulfillment of the requirements for the Degree of DOCTOR OF PHILOSOPHY in Chemistry.

Department of Chemistry

$\begin{array}{ll}\text { Dissertation Advisor: } & \text { Dr. Shiyue Fang } \\ \text { Committee Member: } & \text { Dr. Haiying Liu } \\ \text { Committee Member: } & \text { Dr. Ashutosh Tiwari } \\ \text { Committee Member: } & \text { Dr. Brian Barkdoll } \\ & \\ \text { Department Chair: } & \text { Dr. Cary Chabalowski }\end{array}$ 


\section{Table of Contents}

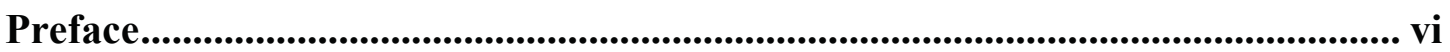

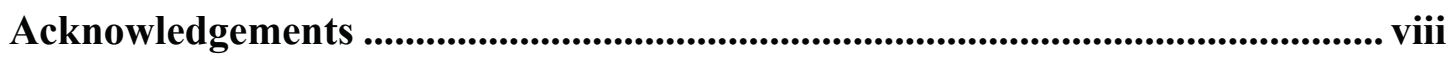

Abstract...................................................................................................... iix

Chapter 1 Introduction to Oligodeoxynucleotides Purification ..............................1

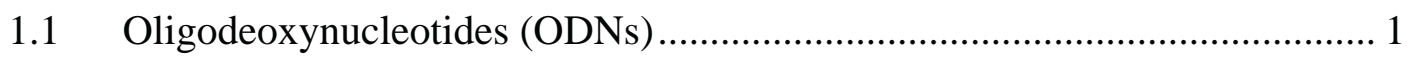

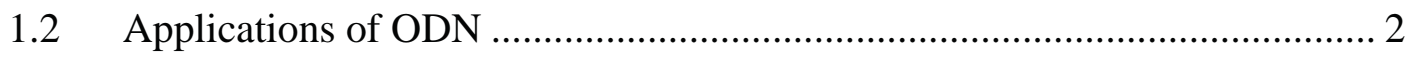

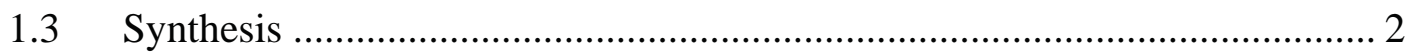

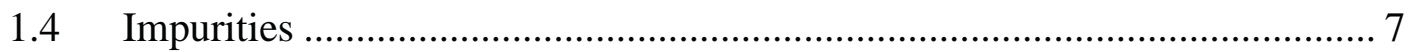

1.5 Current Purification Methods................................................................ 8

1.6 Our Catching by Polymerization ODN Purification Methods ..................... 11

1.6.1 First Method: Catching Failure Sequences by Polymerization .................. 11

1.6.2 Second Method: Catching Full-length Sequences by Polymerization ....... 15

1.7 Advantages of Our New Purification Methods ...................................... 19

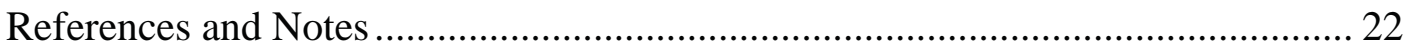

Chapter 2 Synthetic Oligodeoxynucleotide Purification by Capping Failure Sequences with a Methacrylamide Phosphoramidite Followed by

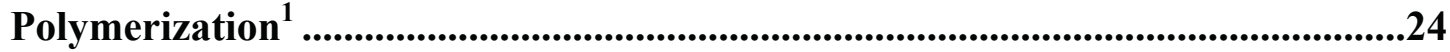

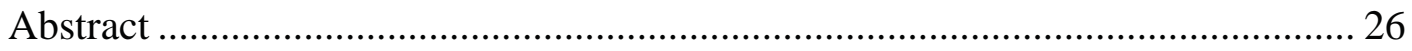

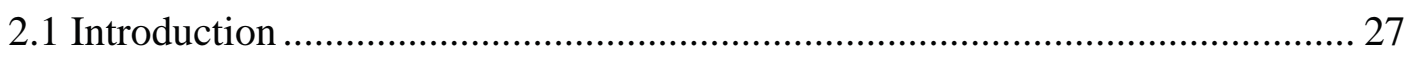

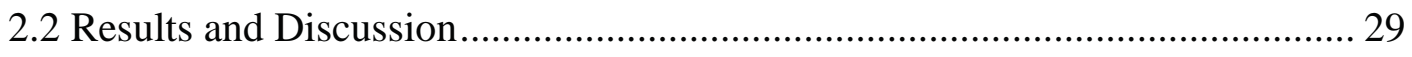

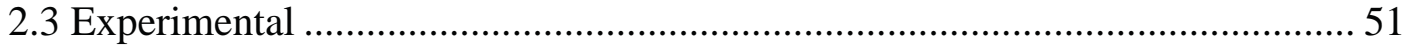

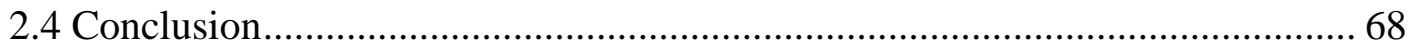

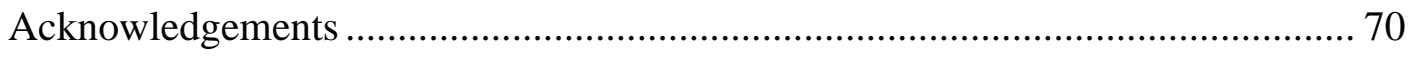

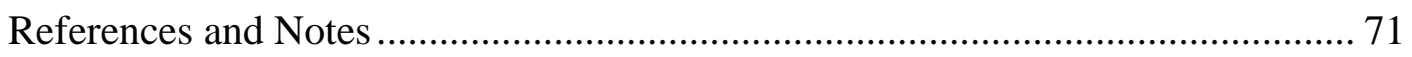

Chapter 3 A Highly Convenient Procedure for Oligodeoxynucleotide Purification ${ }^{1}$

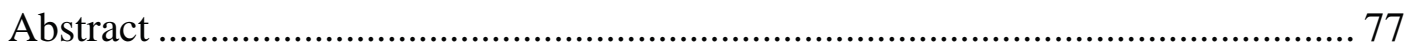

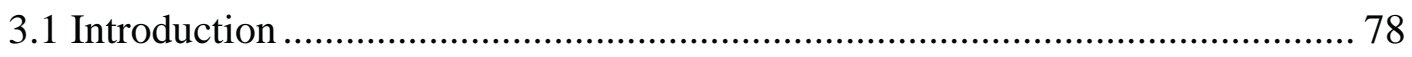

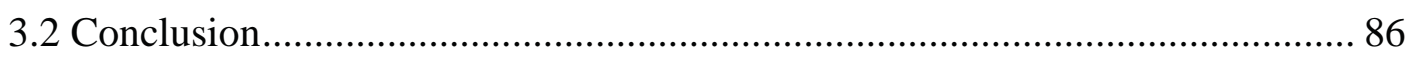




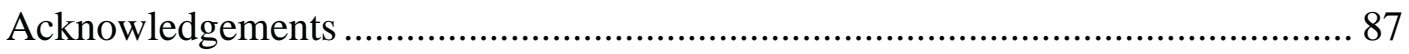

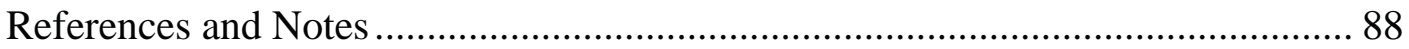

Chapter 4 Methacrylation Phosphoramidite with Acid-Cleavable Linker for Eco-Friendly Synthetic Oligodeoxynucleotide Purification ${ }^{1}$....................................91

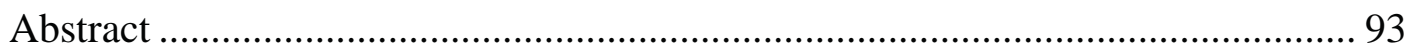

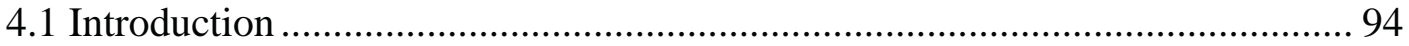

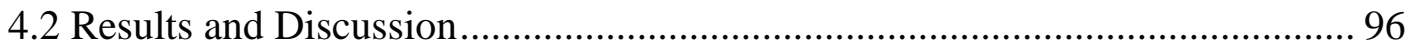

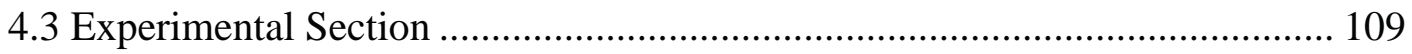

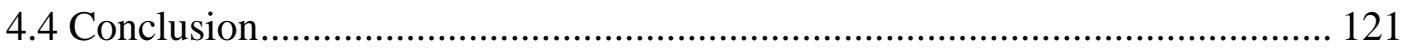

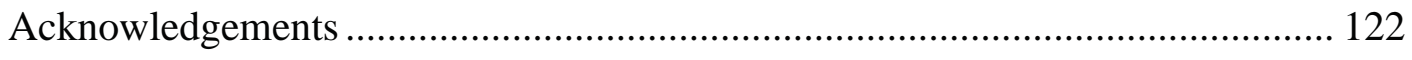

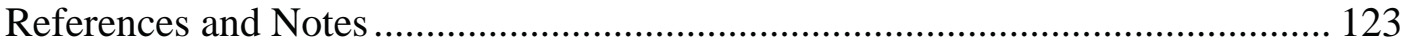

Chapter 5 Introduction to Peptide Purification ..........................................................126

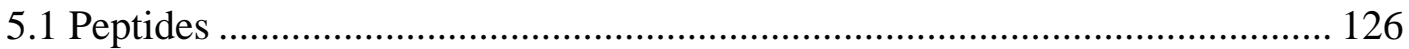

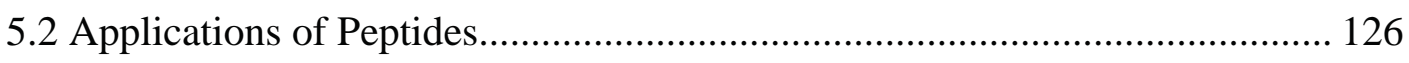

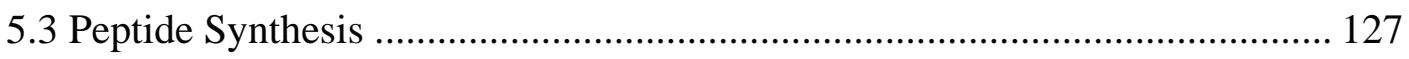

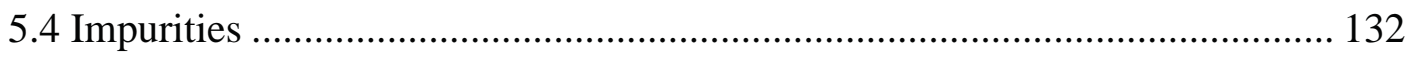

5.5 Current Methods of Purification ................................................................. 133

5.6 Our Peptide Purification Method ................................................................. 133

5.7 Advantages of Our New Peptide Purification Method....................................... 137

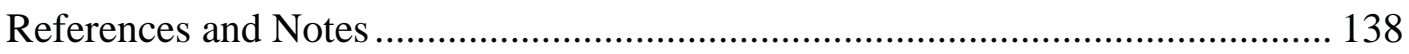

Chapter 6 Purification of Synthetic Peptides Using a Catching Full-Length Sequence by Polymerization Approach ${ }^{1}$.......................................................................140

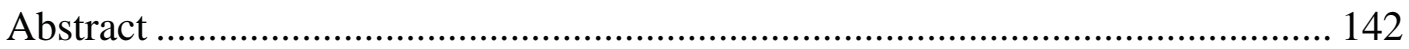

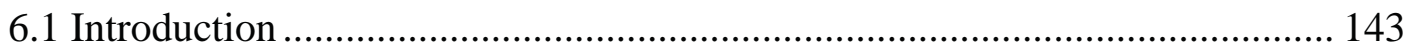

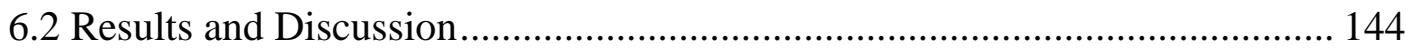

6.3 Experimental Section ............................................................................ 151

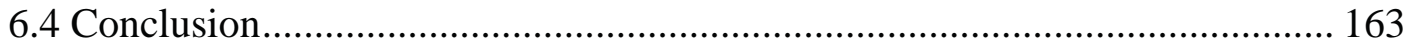

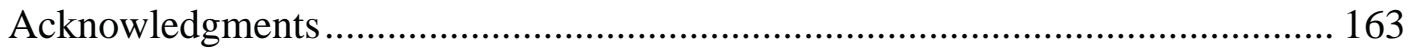

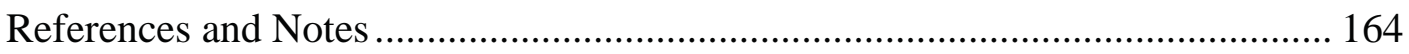

Chapter 7 Future Research Plan ...................................................................................169

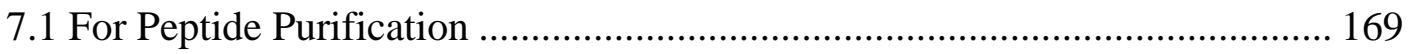

7.2 For ODN and RNA Purification................................................................. 171 
References and Notes

Appendix A Supporting Information for Chapter 2 ..............................................174

Appendix B Supporting Information for Chapter 3...........................................224

Appendix C Supporting Information for Chapter 4 ..........................................233

Appendix D Supporting Information for Chapter 6 ...........................................258

Appendix E Permission from Publisher for Chapter 2 ......................................274

Appendix F Permission from Publisher for Chapter 3 ..........................................276

Appendix G Permission from Publisher for Chapter 6......................................285 


\section{Preface}

The initial ideas of all research projects in this dissertation were conceived by Dr. Shiyue Fang. More than $85 \%$ of the experiments and data analysis in this dissertation were carried out by Mr. Durga P. Pokharel under the supervision of Dr. Shiyue Fang. All writing of this dissertation is carried out by Durga P. Pokharel and revised by Dr. Shiyue Fang.

In chapter 2, the enzyme digestion essay experiments were carried out by Dr. Yinan Yuan. One compound for ODN purification is synthesized by Suntara Fueangfung. The content in chapter 2 was previously published in RSC Advances, 2014, 4, 8746-8757,

In chapter 3, all experiments are carried out by Durga P. Pokharel. The content in Chapter 3 was previously published in The Open Organic Chemistry Journal, 2014, 8, 15-18

All experiments in chapter 4 are carried out by Durga P. Pokharel. The content in chapter 4 will be submitted to a peer reviewed journal for publication.

In chapter 6, Dr. Mingcui Zhang did $40 \%$ of experiments. At the beginning during the first submission for publication, Dr. Mingcui Zhang had more contribution than Durga P. Pokharel. At re-submission, we added more datas the reviews requested. At that stage, the contribution of Durga became more than Dr. Mingcui. At that time, we did not change authorship so Dr. Mingcui Zhang is first author in 
chapter 6. The content in chapter 6 was previously published in Organic Letters, 2014, 16, 1290-1293, 


\section{Acknowledgements}

I am thankful to Dr. Shiyue Fang under whose guidance I accomplished this dissertation. It would be impossible to complete this long journey without his guidance and support. I learnt how to enjoy our work from him which is the major spirit to make working environment better. He is always interested to listen our problem and find ways to solve them.

I am grateful for the support from my present and former lab members Suntara Fueangfung (Boat), Ashok Khanal, Shahien Shahsavari, Dr. Yinan Yuan, Dr. Xi Lin (Sissi), Dr. Mingcui Zhang, Dr. Bin Cao who have been very supportive through my study. I would like to thank fellow graduate students, staff and faculty of chemistry department for their support and help.

I would like to thank my dissertation committee members; Dr. Haiying Liu, Dr. Ashuthosh Tiwari and Dr. Brian Barkdoll. I would like to appreciate their constructive suggestion and motivation.

At last but not least there is a hand of my parents and family to accomplish this work. Though my father and mother are uneducated they always encouraged me to work hard to get higher education. I would like to thank my wife, my brothers and sisters for their support during this journey. 


\begin{abstract}
Synthetic oligonucleotides and peptides have found wide applications in industry and academic research labs. There are $\sim 60$ peptide drugs on the market and over 500 under development. The global annual sale of peptide drugs in 2010 was estimated to be $\$ 13$ billion. There are three oligonucleotide-based drugs on market; among them, the FDA newly approved Kynamro was predicted to have a $\$ 100$ million annual sale. The annual sale of oligonucleotides to academic labs was estimated to be $\$ 700$ million. Both bio-oligomers are mostly synthesized on automated synthesizers using solid phase synthesis technology, in which nucleoside or amino acid monomers are added sequentially until the desired full-length sequence is reached. The additions cannot be complete, which generates truncated undesired failure sequences. For almost all applications, these impurities must be removed. The most widely used method is HPLC. However, the method is slow, expensive, laborintensive, not amendable for automation, difficult to scale up, and unsuitable for high throughput purification. It needs large capital investment, and consumes large volumes of harmful solvents. The purification costs are estimated to be more than $50 \%$ of total production costs. Other methods for bio-oligomer purification also have drawbacks, and are less favored than HPLC for most applications.
\end{abstract}

To overcome the problems of known biopolymer purification technologies, we have developed two non-chromatographic purification methods. They are (1) catching failure sequences by polymerization, and (2) catching full-length sequences 
by polymerization. In the first method, a polymerizable group is attached to the failure sequences of the bio-oligomers during automated synthesis; purification is achieved by simply polymerizing the failure sequences into an insoluble gel and extracting full-length sequences. In the second method, a polymerizable group is attached to the full-length sequences, which are then incorporated into a polymer; impurities are removed by washing, and pure product is cleaved from polymer. These methods do not need chromatography, and all drawbacks of HPLC no longer exist. Using them, purification is achieved by simple manipulations such as shaking and extraction. Therefore, they are suitable for large scale purification of oligonucleotide and peptide drugs, and also ideal for high throughput purification, which currently has a high demand for research projects involving total gene synthesis.

The dissertation will present the details about the development of the techniques. Chapter 1 will make an introduction to oligodeoxynucleotides (ODNs), their synthesis and purification. Chapter 2 will describe the detailed studies of using the catching failure sequences by polymerization method to purify ODNs. Chapter 3 will describe the further optimization of the catching failure sequences by polymerization ODN purification technology to the level of practical use. Chapter 4 will present using the catching full-length sequence by polymerization method for ODN purification using acid-cleavable linker. Chapter 5 will make an introduction to peptides, their synthesis and purification. Chapter 6 will describe the studies using the catching full-length sequence by polymerization method for peptide purification. 


\section{Chapter 1 \\ Introduction to Oligodeoxynucleotides Purification}

\subsection{Oligodeoxynucleotides (ODNs)}

Oligodeoxynucleotides (ODNs) $\mathbf{1 . 1}$ are short single-stranded deoxyribonucleic acid or DNA. They are comprised of four nucleosides deoxyadenosine (dA), thymidine (dT), deoxycytidine (dC), and deoxyguanosine (dG). Each nucleoside is comprised of a 2'-deoxyribose sugar and nucleobase such as adenine, thymine, cytosine and guanine. The nucleosides with phosphate group are known as nucleotides. Multiple nucleosides linked together by phosphate diester linkages form ODN. Usually, ODN refers to deoxyribonucleic acids with lengths of

15 to 200 nucleotide units.

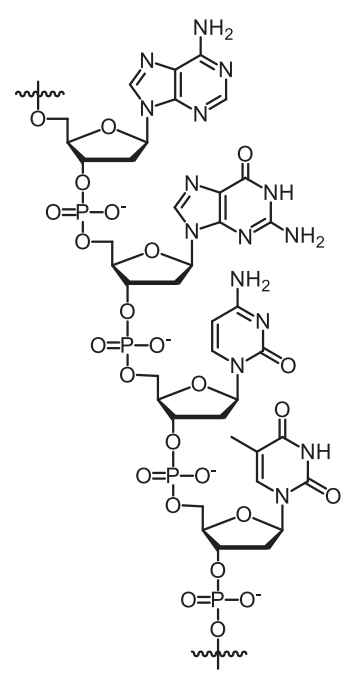

1.1

Figure 1.1. Structure of ODN 


\subsection{Applications of ODN}

Synthetic ODNs encompass a wide range of applications in the area of genomics, medical diagnosis, forensic science and nanotechnology.[1] Recently, synthetic ODNs have been booming in therapeutics and antisense therapy.[2] The antisense therapy is based on specific binding of ODN sequences on specific gene sequence and is used to treat diseases caused by genetic disorders.[3] Currently, three oligonucleotide based drugs are on the market. Kynamro is the newly approved drug by FDA which was predicted to have a $\$ 100$ million annual sale [4]. In academic lab, the annual sale of oligonucleotide is $\$ 700$ million [5]. More than 60 drugs based on oligonucleotide are in various stages of preclinical and clinical testing for treating diseases such as cancer, viral infection and diabetes. In addition synthetic ODNs have been widely used as primers for DNA sequencing and polymerase chain reaction (PCR).[6]

\subsection{Synthesis}

The most widely used method for ODN synthesis is the phosphoramidite chemistry. Usually, the synthesis is carried out on a solid support. During synthesis the first nucleoside is pre-anchored to the support, and nucleoside phosphoramidite monomers are added sequentially one at a time. Each addition requires four steps,[7] which is called a synthetic cycle. The four steps are detritylation, coupling, capping and oxidation. At the end of synthesis, the ODNs are cleaved from the solid support 
and fully deprotected. The following paragraphs give detailed description of the four steps and the most widely used conditions for cleavage and deprotection.

\subsubsection{Detritylation}

This is the first step of ODN synthesis cycle. As shown in scheme 1.1, it converts 1.2 to 1.3. The 5'-OH group of nucleoside phosphoramidite is protected with the 4,4'-dimethoxytrityl (DMTr) group. Thus the growing chain of ODN contains DMTr group at the $5^{\prime}$ end. This group needs to be removed in each cycle before the coupling step. This group is acid labile and is usually removed by $3 \%$ trichloroacetic acid in dichloromethane. Once the DMTr group is removed, $5^{\prime}-\mathrm{OH}$ group $\mathbf{1 . 3}$ is generated and the solid support is ready for the coupling step in the cycle. 

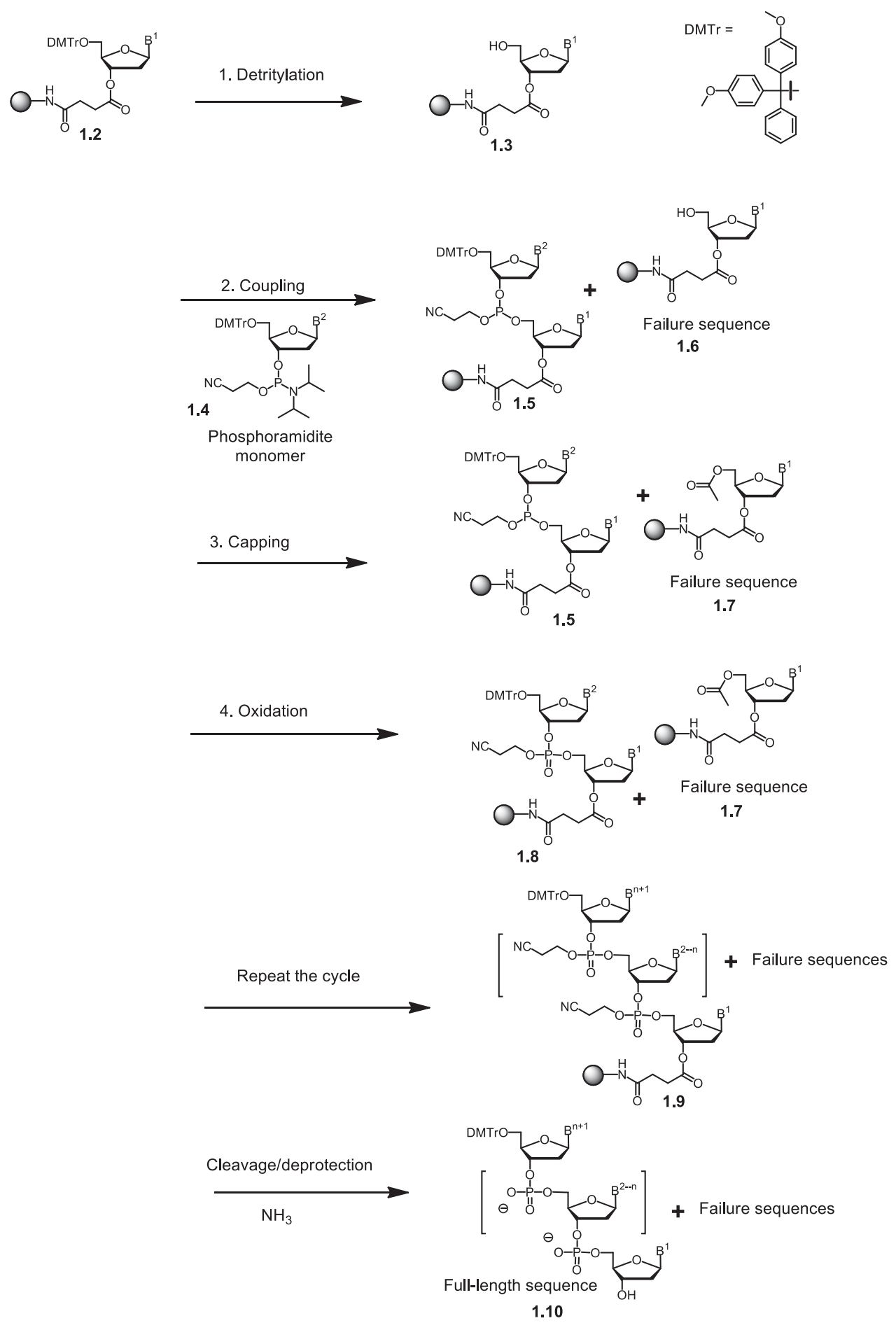

Scheme 1.1. Oligodeoxynucleotide synthesis cycle using phosphoramidite chemistry 


\subsubsection{Coupling}

After detritylation, the next step is coupling in which $\mathbf{1 . 3}$ reacts with phosphoramidite monomer $\mathbf{1 . 4}$ to give $\mathbf{1 . 5}$ (Scheme 1.1). The nucleoside phosphoramidite $\mathbf{1 . 4}$ and activator $1 H$-tetrazole or 4,5-dicyanoimidazole are delivered to the synthesis column, which contains the solid support. The activator protonates the diisopropylamino group of nucleoside phosphoramidite making it a better leaving group. The free $5^{\prime}-\mathrm{OH}$ group readily displaces the leaving group and the phosphite triester $\mathbf{1 . 5}$ is generated.

\subsubsection{Capping}

After coupling, the next step is capping. The coupling step cannot be $100 \%$ complete. Some nucleosides with free 5'-OH group (1.6) will remain unreacted. If they are not blocked right after coupling, they will continue to elongate in the next synthetic cycles giving what is called deletion sequences. Deletion sequences are very difficult to remove during purification. As a result, these $5^{\prime}-\mathrm{OH}$ groups are capped with large excess of acetic anhydride to give the failure sequences 1.7.

\subsubsection{Oxidation}

After capping, an oxidation step is executed. The newly formed triester linkage in $\mathbf{1 . 5}$ in the coupling step is unstable under next detritylation conditions, which is acidic. It must be converted to the stable phosphate triester 1.8. The step is achieved by treating with iodine in water in the presence of pyridine. After oxidation, the cycle is completed, and this finishes addition of one nucleoside phosphoramidite 
monomer, and the ODN grows one nucleotide longer. The synthesizer repeats the cycles until the desire ODN length is reached.

\subsubsection{Cleavage}

In phosphoramidite chemistry, the succinyl linker connects $3^{\prime}$ end of ODN to the solid support. This linker is stable to all the reagents used during solid phase synthesis. As the desired length of ODN is reached, the linker is usually cleaved with concentrated ammonium hydroxide solution at room temperature. The cleavage reaction can be carried out either on the synthesizer automatically or off the synthesizer manually.

\subsubsection{Deprotection}

The exocyclic amino groups on the heterocyclic bases (A, C, and G) are nucleophilic and should be protected thoughout the ODN synthesis. The most commonly used protecting groups for A and C are benzoyl and for G isobutyryl. These protecting groups can be removed with concentrated ammonium hydroxide solution at $55{ }^{\circ} \mathrm{C}$ for 15 hours. In some cases, the Ultramild protecting groups are used and are attached to the monomers. The Ultramild protecting group for A, C and $\mathrm{G}$ are phenoxyacetyl, acetyl and isopropyl phenoxyacetyl, respectively. These protecting groups can be readily removed by concentrated ammonium hydroxide solution at room temperature in 2 hours.

During synthesis, the phosphate groups are also protected. The mostly used group is the 2-cyanoethyl group. The protecting groups are usually easy to remove. 
They usually fall off during cleavage via a $\beta$-elimination mechanism caused by a base such as ammonia.

\subsection{Impurities}

The ODNs cleaved from the synthesizer and full deprotected contain impurities. They must be removed before most applications. The impurities are classified into three different types.

The first type is called truncated failure sequences. They are generated in each synthetic cycle due to incomplete coupling of nucleoside phosphoramidite monomer. The amount of failure sequences in crude ODN depends on the sequence, scale, and length of ODNs. For example, in a normal 20-mer ODN synthesis, failure sequences constitute 30-60\% ODN content.[8] The failure sequences have similar physical properties as the full-length ODN which make them difficult to be separated.

The second type of impurities is small organic molecules from the protecting groups. These molecules are neutral. They are easy to remove from the negatively charged full-length sequences. These small molecules are soluble in ethanol or $n$ butanol while the full-length ODNs are insoluble. Simple precipitation can remove these small organic molecules.

The third types of impurities are deletion sequences, addition sequences and sequences resulted from depurination. Deletion sequences are resulted from incomplete capping and incomplete de-tritylation. Addition sequences are resulted from premature de-tritylation. The deletion and addition sequences are very difficult 
to remove. They should be minimized by careful tuning synthesis conditions. Based on the above analysis, the major task of ODN purification is the removal of failure sequences.

\subsection{Current Purification Methods}

Currently there are several known methods to purify ODN. They include reversed-phase (RP) high performance liquid chromatography (HPLC), anionexchange HPLC, gel electrophoresis, fluorous affinity purification, RP cartridge chromatography and biotin-avidin based affinity purification. The following paragraphs describe the principle, advantages and disadvantages of these methods.

\subsubsection{RP HPLC}

The principle of this method is based on the hydrophobic interaction between ODN and stationary phase. The stationary phase contains long hydrophobic chain. More hydrophobic ODNs interact with the stationary phase more strongly and elute slowly while less hydrophobic impurities move faster. The ODNs with fluorescent dyes and DMTr groups are more hydrophobic which makes the purification easier.[6] This method is very efficient and is most widely used in industrial processes. It can separate ODNs of similar size on the basis of hydrophobicity. However, the ODNs which form stable secondary structure is difficult to separate by RP HPLC because the ODN elutes as a broad peak or multiple peaks. In addition the method needs expensive instruments and columns. It also requires large volume of solvents and is 
highly labor-demanding.[9] As a result, RP HPLC is difficult to scale up, and is expensive for large-scale ODN purification.

\subsubsection{Anion-Exchange HPLC}

In anion-exchange HPLC, the stationary phase contains positively charged functional groups. The mobile phase is usually $25 \mathrm{mM}$ Tris/Cl, $1 \mathrm{mM}$ EDTA and 25 $\mathrm{mM}$ Tris/Cl $1 \mathrm{mM}$ EDTA, $1.0 \mathrm{M} \mathrm{NaCl}$. ODN is negatively charged. It has rich interaction with the stationary phase. The interactions are different for different ODN sequences. As a result, they are eluted by the mobile phase with different rate. Although this method can purify short ODN with good resolution, it needs expensive instruments, column and large volume of solvents. It is also labor intensive. The purified ODN needs further desalting because the eluent contains non-vaporizable salts.

\subsubsection{Gel-Electrophoresis}

In gel-electrophoresis, an electrical field is applied across a gel. The ODN is loaded at one side of the gel. Because ODN is negatively charged, it travels from high potential end of the electrical field to the low potential end. Different ODNs have different mass to charge ratios, and they travel with different speeds. As a result, they are separated in the process. This method can produce ODN in high levels of purity but is only suitable for very small scale purification.[6] It cannot be scaled up for large scale purifications. 


\subsubsection{Fluorous Affinity Purification}

During ODN synthesis, the full-length sequences are tagged with a molecule containing multiple fluorine atoms. The failure sequences are not tagged. During purification, the crude ODN solution is passed through a column containing fluorinated stationary phase. Due to the strong attraction forces between fluorinated molecules on the full-length sequences and the stationary phase, the full-length sequences have a much longer retention time than the failure ones.[8] As a result, the full-length sequences are easily separated from failure ones and other impurities. The method can purify ODN in high levels of purity but it has disadvantages. It is expensive to scale up and fluorous column is not reusable.

\subsubsection{RP Cartridge}

RP cartridge is a disposable column that contains C-18 alkyl group functionalized silica gel. The surface of the silica gel is hydrophobic. During ODN synthesis, the DMTr group on the full-length sequences is not removed. The failure sequences and other molecules do not contain DMTr group. During purification, the crude ODN solution is passed through the column. Due to strong hydrophobic attraction, the full-length sequences are eluted much slower than the failure ones, and the two are separated.[10] This method is cheap and convenient for short ODNs. However, this purification method is not suitable for longer ODNs. The hydrophobicity effect of the DMTr groups ultimately diminishes with increasing ODNs size. This results in a less efficient purification process overall. Another 
drawback of this method lies with the extremely susceptible characteristic of the $5^{\prime}-$ DMTr group which results in a general low yield.[8]

\subsubsection{Biotin-Avidin Based Affinity Purification}

The principle of this method is based on the interaction between biotin and avidin. A biotin molecule is tagged with full-length ODN during the synthesis. The full-length ODN with biotin binds with avidin while the failure sequences which lack biotin tag are washed away. The desired ODN is cleaved from avidin microspheres.[11-13] This method is convenient to purify small quantities of ODN. However, it has disadvantages. It requires harsh conditions to cleave ODN from avidin-biotin linkage and is expensive because of the high cost associated with avidin which is not reusable.

\subsection{Our Catching by Polymerization ODN Purification Methods}

To overcome the problems of known ODN purification technologies, we have developed two new methods. Both methods are based on a new concept which is catching by polymerization. The following paragraphs briefly describe the principle of the two methods and their advantages over known methods.

\subsubsection{First Method: Catching Failure Sequences by Polymerization}

The first method is called catching failure sequences by polymerization. During automated synthesis, the failure sequences are capped with a phosphoramidite that contain a polymerizable group. The full-length sequences are not capped with 
this phosphoramidite. As a result, the failure sequences contain a polymerizable group while the full-length sequences do not. During purification, the crude ODN is subjected into a polymerization condition. The failure sequences are incorporated into a polymer. The full-length sequences remain in solution. Extraction followed by $n \mathrm{BuOH}$ precipitation gives pure $\mathrm{ODN}$. The polymerizable group we used is the methacrylamide group, which can be incorporated into a polyacrylamide gel. Four capping phosphoramidites (1.11-1.14) have been tested so far. All of them worked great. However 1.11-1.12 are preferred because they are easier to synthesize and cheaper. Scheme 1.2 illustrates how to cap the failure sequences with a polymerizable phosphoramidite while leaving the full-length sequences un-capped.
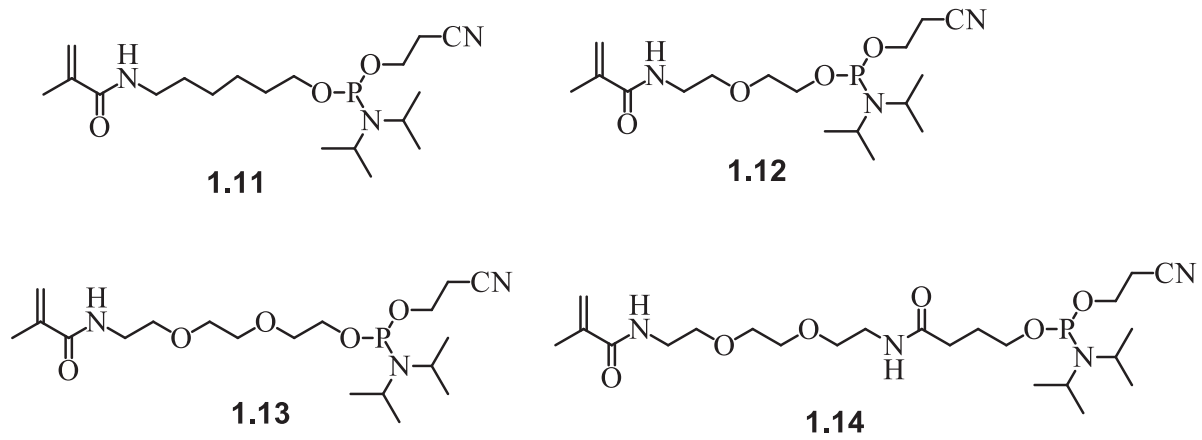

Figure 1.2. Capping reagents for catching failure sequences

As shown in Scheme 1.2, after the coupling step, the failure sequences $\mathbf{1 . 6}$ are capped with polymerizable phosphoramidite 1.12. Instead of using acetic anhydride, the polymerizable phosphoramidite $\mathbf{1 . 1 2}$ is delivered to the column right after the coupling step. The failure sequences 1.6 react with 1.2 to give 1.15 , which after the 
oxidation step, becomes 1.16. The failure sequences $\mathbf{1 . 1 6}$ now contain a methacrylamide polymerizable group. After synthesis, cleavage and deprotection, all failure sequences are depicted as $\mathbf{1 . 1 7}$ which contains a polymerizable group while full-length sequences $\mathbf{1 . 1 0}$ do not. The crude ODN is subjected into radical polymerization conditions. $N, N$-Dimethylacrylamide is used as the polymerization monomer and $N, N$-methylenebisacrylamide is used as a cross-linker. The polymerization is initiated with ammonium persulfate (APS) and $N, N, N^{\prime}, N^{\prime}-$ tetramethylethylenediamine (TMEDA). The failure sequences are incorporated into the polyacrylamide gel $\mathbf{1 . 1 8}$ (scheme 1.3). The full-length ODN $\mathbf{1 . 1 0}$ remains in the gel-matrix. The full-length ODN is extracted with water. At this stage, the full-length sequence is still contaminated with small molecules which are resulted from protecting groups. However, these small molecules are easily removed by precipitation of $\mathrm{ODN}$ from $\mathrm{NH}_{4} \mathrm{OH}$ solution with $n \mathrm{BuOH}$ (Scheme 1.3). This technology is useful to purify short ODNs because the failure sequences needs to be capped in each synthesis cycle and the capping agent is more expensive than acetic anhydride. We used this technology to purify different ODNs including short (20mer) and relatively longer ones (61-mer). This technology also works well to purify at large scales. We tested $3 \mu \mathrm{mol}$ purification, the results were excellent. The ODNs purified by this method has high purity and good recovery yield $(>80 \%)$. 

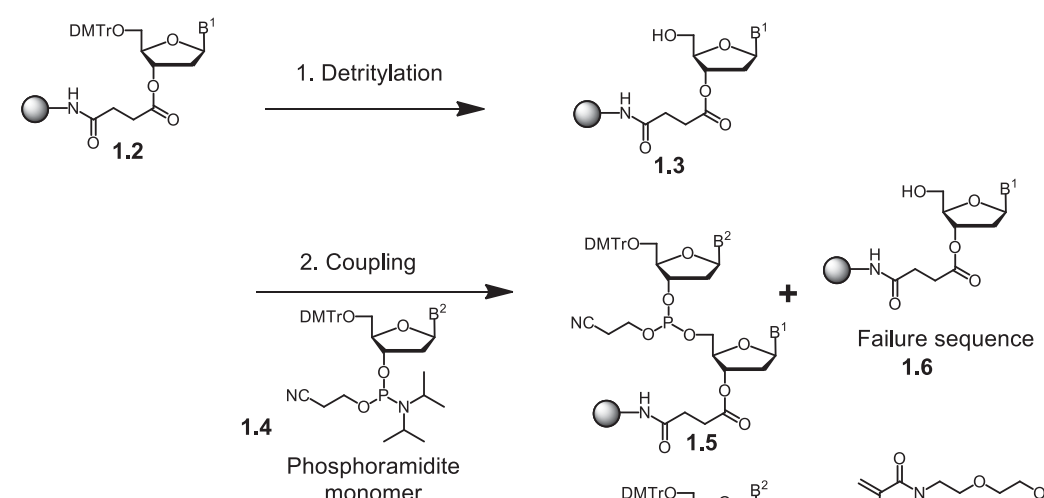

monomer
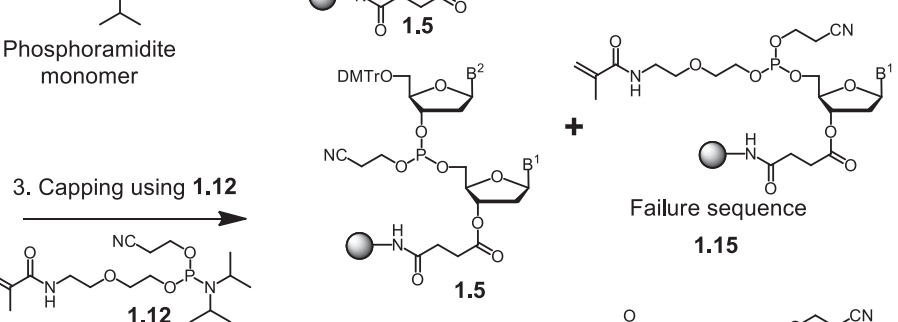

1.15
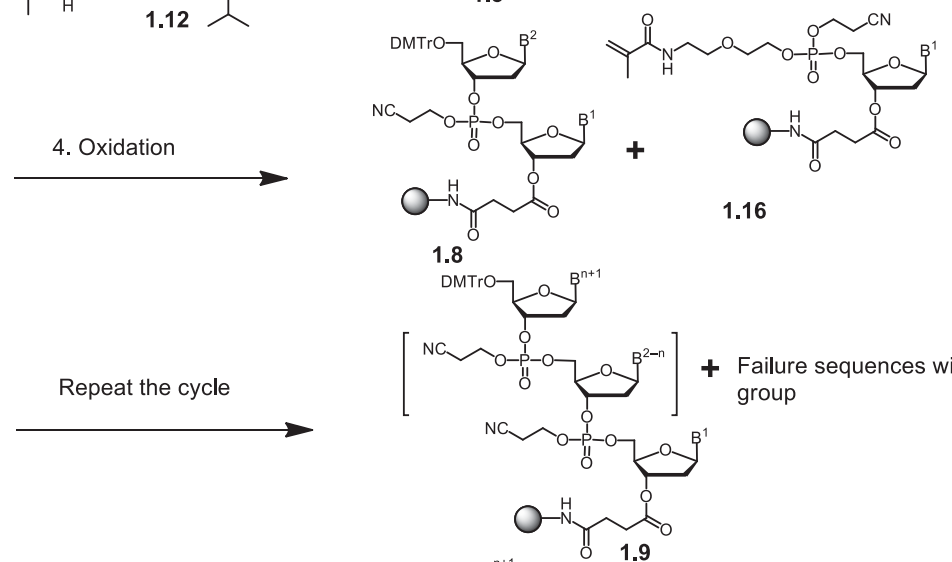

+ Failure sequences with polymerizable
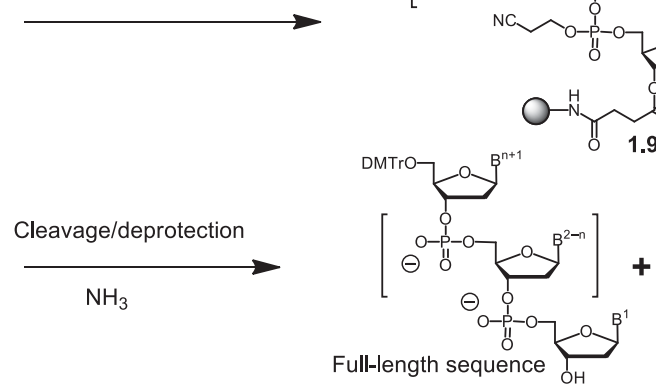

1.10

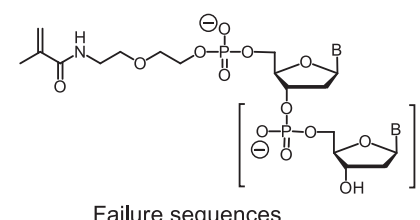

1.17

Scheme 1.2. Acrylation of failure sequences using 1.12 during ODN synthesis 

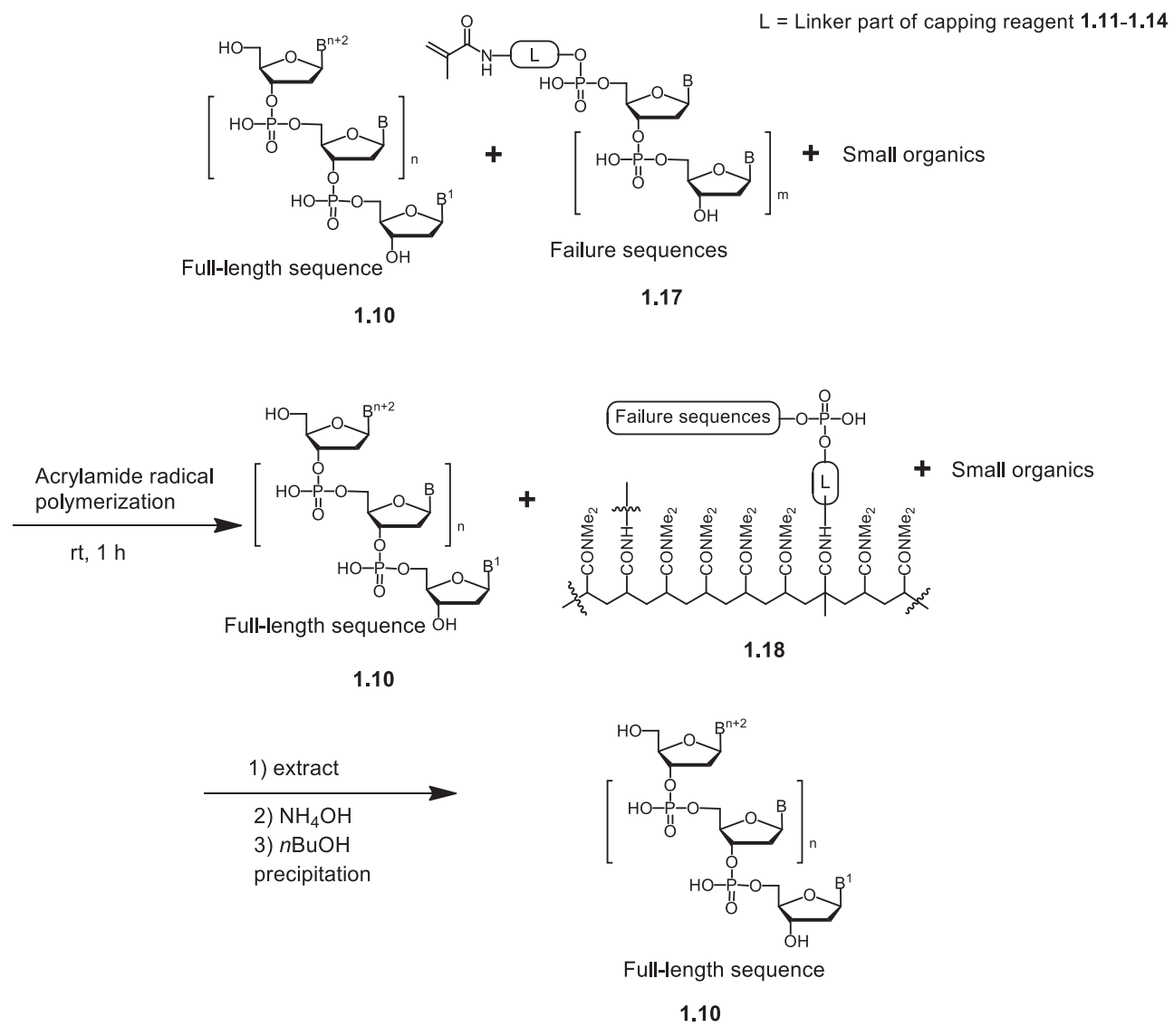

Scheme 1.3. Catching failure sequences by polymerization

\subsubsection{Second Method: Catching Full-length Sequences by Polymerization}

The second method is called catching full-length sequences by polymerization. In this method, the full-length sequences are tagged with a polymerizable phosphoramidite. The failure sequences are capped with acetic anhydride. Thus the full-length sequences have a polymerizable group while failure sequences do not. The crude ODN is subjected to polymerization. The full-length sequences are incorporated into a polymer. The failure sequences remain in the gelmatrix, which are removed by washing. The full-length ODN is cleaved from the gel 
using $80 \%$ acetic acid followed by extraction. Pure ODN is obtained by $n \mathrm{BuOH}$ precipitation from $\mathrm{NH}_{4} \mathrm{OH}$. The polymerizable tagging agent 1.19 used to catch fulllength is used in our examples. It contains an alkyl trityl ether linkages, which is readily cleavable under weekly acidic conditions. The polymerizable group is the methacrylamide.

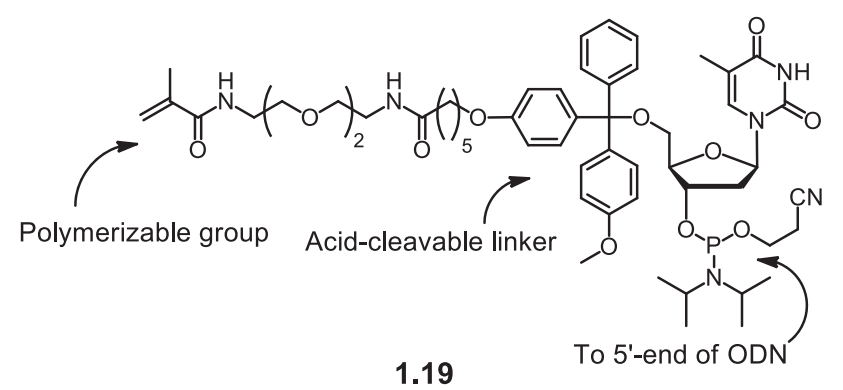

Figure 1.3. Polymerizable tagging phosphoramidite with an acid cleavable linker

Scheme 1.4 describes the procedure for tagging full-length sequences with a polymerizable linker 1.19. During ODN synthesis, failure sequences are capped with acetic anhydride in each synthesis cycle. At the end of synthesis, the full-length sequence is tagged with the polymerizable phosphoramidite 1.19 . The $5^{\prime}$ end of fulllength ODN then contains a polymerizable group 1.20. After cleavage and deprotection, the crude ODN contains a mixture of full-length ODN 1.21, which contains a polymerizable group and the failure sequences, which do not contain a polymerizable group. 


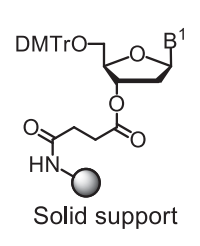

1.2

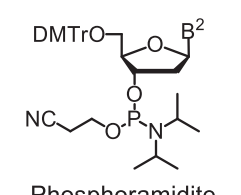

Phosphoramidite monomer

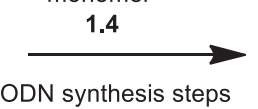

ODN synthesis steps
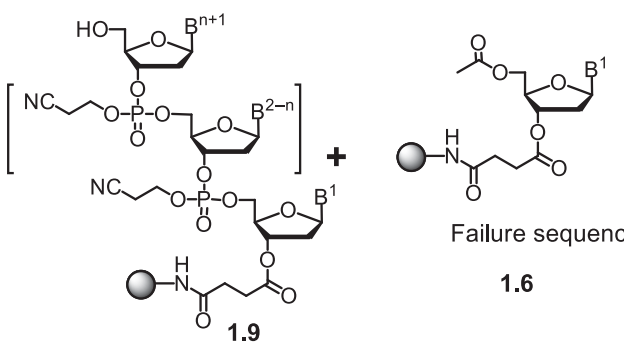

Failure sequence

1.6

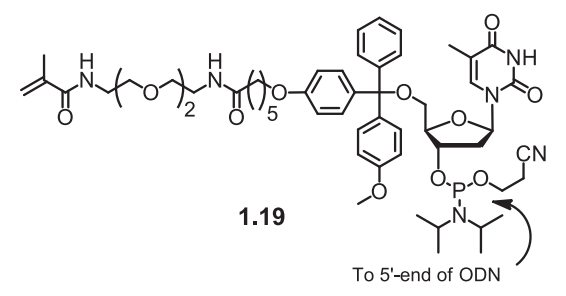

Tagging with polymerizable phosphoramidite

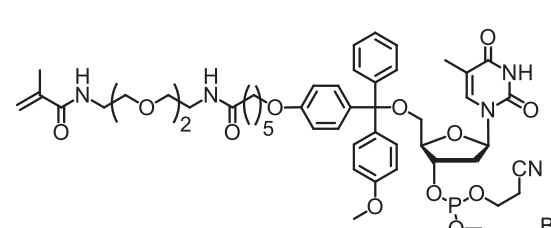

$\underset{ }{\longrightarrow}$

Cleavage/deprotection
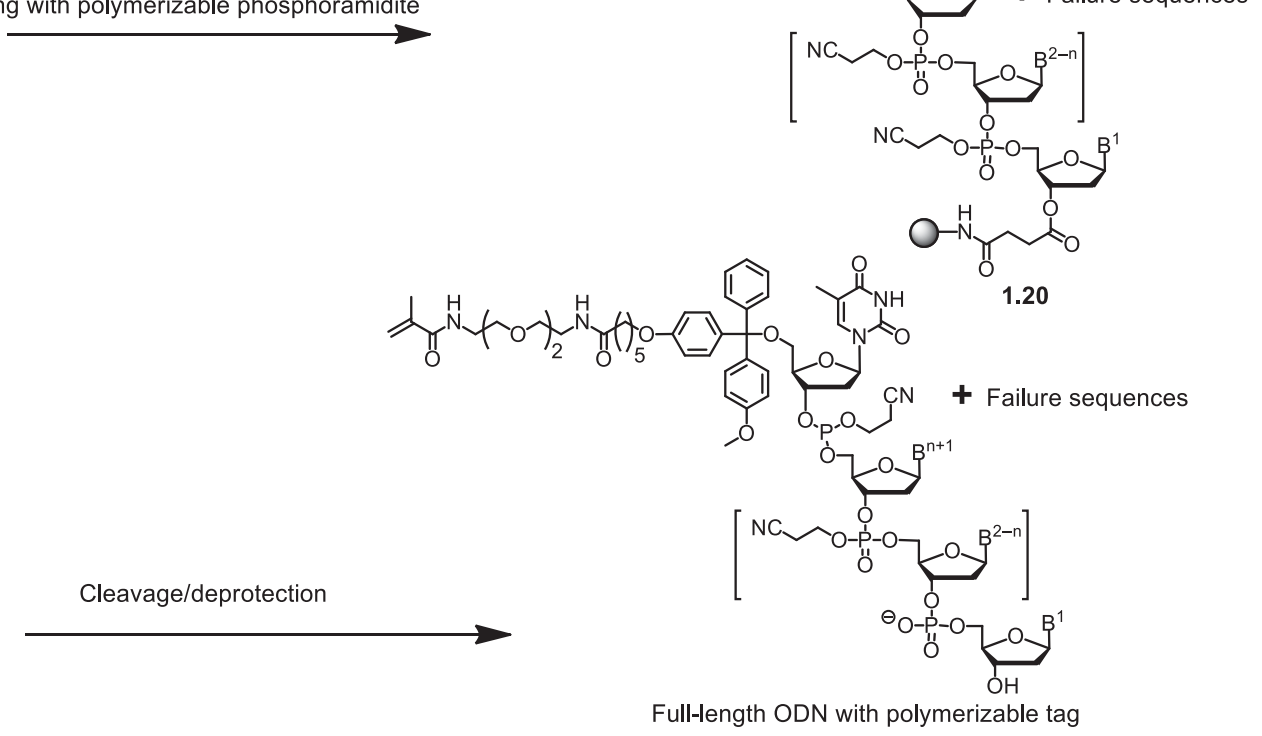

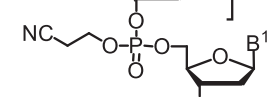

1.21

Scheme 1.4. Tagging of full-length sequences during ODN synthesis

The crude ODN is subjected into radical polymerization conditions which are the same as described for the first purification method. The full-length sequences are 
incorporated into the polyacrylamide gel $\mathbf{1 . 2 4}$ (Scheme 1.5). The failure sequences and other impurities remain in the gel-matrix. The failure sequences and other impurities are washed away. The full length ODN is cleaved with $80 \%$ acetic acid. The full-length ODN is further extracted with water. Pure ODN is obtained after $n \mathrm{BuOH}$ precipitation from $\mathrm{NH}_{4} \mathrm{OH}$.

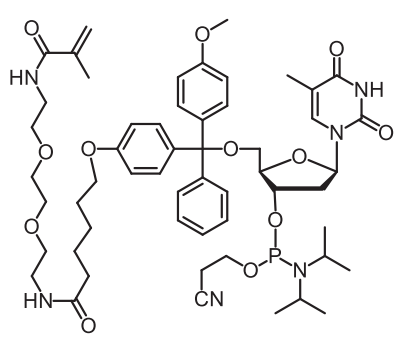

1.22

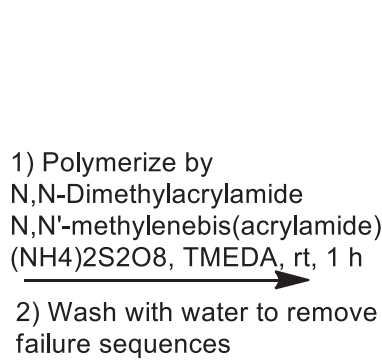

1) Polymerize by

$\mathrm{N}, \mathrm{N}$-Dimethylacrylamide

$\mathrm{N}, \mathrm{N}^{\prime}$-methylenebis(acrylamide)

2) Wash with water to remove

failure sequences
Synthesize oligo using 1.22 to tag

full-length

sequences

Following Scheme 1.4

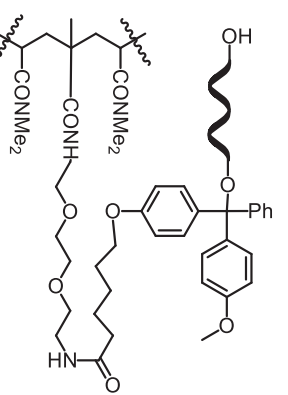

full-length sequences

on polymer

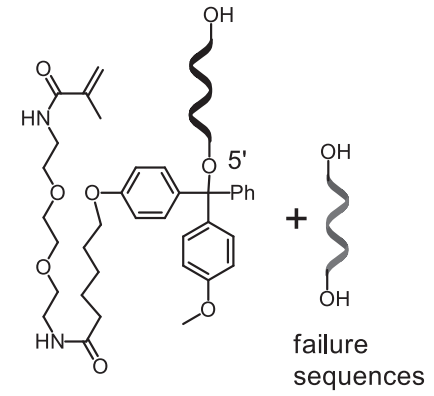

full-length sequences

1.23

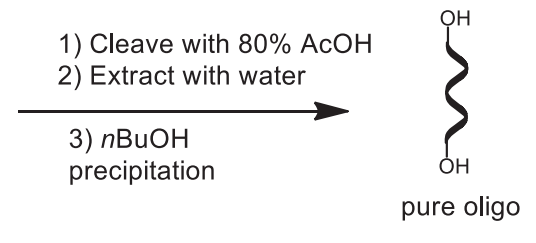

1.24

Scheme 1.5. Purification of ODN by catching full-length

Using this technology, the polymerizable tagging agent is used only once at the last synthetic cycle of ODN synthesis. So the method is suitable to purify longer ODNs. In our examples, we used this technology to purify ODNs as long as (151 $m e r)$ and at scales as large as $50 \mu \mathrm{mol}$. The purity and recovery yields are very good. We believe that the method can be further scaled up easily. 


\subsection{Advantages of Our New Purification Methods}

Our new methods have significant advantages over all known ODN purification technologies. They do not need any chromatography. Purification is achieved by simple manipulations such as mixing, shaking, washing and extraction. They do not require large volumes of solvents and expensive column. The technologies use inexpensive materials and avoid expensive instrumentation. As a result, they are suitable for large scale and high throughput purification. The ODNs purified by the methods have high purity and good recovery yields. The technologies use environmentally benign materials and save a substantial amount of energy, which are usually needed for solvent evaporation using methods such as RP HPLC.

The advantages of our technologies over known are summarized in Table 1.1. 
Table 1.1. Advantages of the our purification techniques over known ones a

\begin{tabular}{|c|c|c|c|c|c|c|c|}
\hline Techniques & Our methods & $\begin{array}{l}\text { RP } \\
\text { HPLC }\end{array}$ & $\begin{array}{l}\text { IX } \\
\text { HPLC }\end{array}$ & PAGE & $\begin{array}{l}\text { Fluorous } \\
\mathrm{b}\end{array}$ & $\begin{array}{l}\text { Biotin- } \\
\text { Avidin }^{c}\end{array}$ & $\begin{array}{l}\mathrm{RP} \\
\text { cartridge }\end{array}$ \\
\hline Expensive instrument & $\mathrm{No}$ & Yes & Yes & Medium & No & No & No \\
\hline Expensive column & No & Yes & Yes & No & Yes $^{d}$ & No & Yes $^{d}$ \\
\hline Expensive materials & No & No & No & No & Yes $^{d}$ & Yes $^{d}$ & Yes $^{d}$ \\
\hline Scalability & Excellent & Medium & Medium & No & No & No & No \\
\hline $\begin{array}{l}\text { High } \\
\text { throughput } \\
\text { purification }\end{array}$ & Yes & No & No & No & No & No & No \\
\hline Desalting & No & No & Yes & No & No & No & No \\
\hline Automation & Yes & No & No & No & No & Possible & No \\
\hline Labor intensive & No & Yes & Yes & Yes & Medium & Medium & Medium \\
\hline Harmful solvent & Minimal & Large & Large & Small & Small & Minimal & Medium \\
\hline Waste to product ratio & $0.1 \mathrm{~K} / 1$ & $200 \mathrm{~K} / 1$ & $200 \mathrm{~K} / 1$ & $50 \mathrm{~K} / 1$ & $0.5 \mathrm{~K} / 1$ & $0.2 \mathrm{~K} / 1$ & $10 \mathrm{~K} / 1$ \\
\hline Energy demand ${ }^{\mathrm{e}}$ & Low & High & High & Low & Low & Low & High \\
\hline Environmental benign & High & Low ${ }^{f}$ & Low ${ }^{f}$ & Low ${ }^{g}$ & Low ${ }^{h}$ & - & Low ${ }^{f}$ \\
\hline Product purity ${ }^{\mathrm{i}}$ & $98-100 \%$ & $\begin{array}{l}80- \\
100 \%\end{array}$ & $\begin{array}{l}80- \\
100 \%\end{array}$ & $\begin{array}{l}80- \\
100 \%\end{array}$ & $\begin{array}{l}85- \\
100 \%\end{array}$ & $90-100 \%$ & $70-100 \%$ \\
\hline $\begin{array}{l}\text { Purity dependence } \\
\text { on loading }\end{array}$ & No & High & High & High & Low & No & High \\
\hline Recovery yield & $80-99 \%$ & $\sim 70 \%$ & $\sim 65 \%$ & $\sim 50 \%$ & $\sim 80 \%$ & $\sim 80 \%$ & $\sim 60 \%$ \\
\hline Purification time $^{\mathrm{j}}$ & Fast & Slow & Slow & Slow & Fast & Medium & Fast \\
\hline $\begin{array}{l}\text { Overall purification } \\
\text { cost } \mathrm{k}\end{array}$ & $\sim 15 \%$ & $100 \%$ & $\sim 120 \%$ & $\sim 400 \%$ & $\sim 200 \%$ & $\sim 250 \%$ & $\sim 70 \%$ \\
\hline
\end{tabular}

a Information is based on our experience. We tried best to be fair. ${ }^{b}$ Refers to fluorous affinity purification. ${ }^{c}$ Refers to biotin-avidin affinity purification. ${ }^{\mathrm{d}}$ Refers to fluorous support, biotin and avidin, or C-18 silica. ${ }^{\mathrm{e}}$ Energy is needed for solvent evaporation. ${ }^{\mathrm{f}}$ Large amount of harmful solvents. ${ }^{\mathrm{g}}$ Large amount of acrylamide. ${ }^{\mathrm{h}}$ Fluorous materials are not environmental benign. ${ }^{\mathrm{i}}$ Purity is dependent on batch scale for known techs but not so for MTU techs. ${ }^{j}$ Solvent evaporation time is not considered. ${ }^{\mathrm{k}}$ Relative to RP HPLC.

A comarision of different techniques for oligonucleotide purification is shown

\section{in Table 1.2.}


Table 1.2. Costs of different ON purification methods ${ }^{a}$

\begin{tabular}{lllll}
\hline \multicolumn{1}{c}{ Cost } & $\begin{array}{l}0.2 \mu \mathrm{mol} \\
(\sim 1 \mathrm{mg})\end{array}$ & $\begin{array}{l}1 \mu \mathrm{mol} \\
(\sim 6 \mathrm{mg})\end{array}$ & $\begin{array}{l}10 \mu \mathrm{mol} \\
(\sim 60 \mathrm{mg})\end{array}$ & $\begin{array}{l}1 \mathrm{~mol} \\
(\sim 6 \mathrm{~kg})\end{array}$ \\
\hline Electrophoresis & $\$ 125^{\mathrm{b}}$ & $\$ 425^{\mathrm{b}}$ & ${ }^{\mathrm{c}}$ & ${ }^{\mathrm{c}}$ \\
\hline RP HPLC & $\$ 45^{\mathrm{b}}$ & $\$ 90^{\mathrm{b}}$ & $\$ 150^{\mathrm{b}}$ & $\mathrm{c}$ \\
\hline Fluorous method & $\$ 18^{\mathrm{d}}$ & $\$ 22^{\mathrm{d}}$ & $\mathrm{c}$ & ${ }^{\mathrm{c}}$ \\
\hline MTU methods & $\$ 9^{\mathrm{e}}$ & $\$ 10^{\mathrm{e}}$ & $\$ 12^{\mathrm{e}}$ & $\$ 1,500^{\mathrm{e}}$ \\
\hline Saving over HPLC & $80 \%$ & $88 \%$ & $92 \%$ & - \\
\hline
\end{tabular}

${ }^{a}$ Based on 20-mer, which has a molecular weight of $\sim 6 \mathrm{~kg} / \mathrm{mol}$.

${ }^{\mathrm{b}}$ Appromxiate Price.

${ }^{\mathrm{c}}$ No information on the web.

${ }^{\mathrm{d}}$ Price estimated from website; [14] labor not included.

${ }^{\mathrm{e}}$ Estimated number; labor not included; not accurate.

The specific details of new ODN purification methods are described in subsequent chapters. Purification of ODN by catching failure sequences is described in chapters 2 and 3. Chapter 2 describes the detailed investigation of this technology while in chapter 3 we highlight the optimization and refinements of this technology for practical use. In chapter 4 we describe the second method of purifications, which is catching full-length ODN by polymerization. An acid-cleavable linker is used in the examples. 


\section{References and Notes}

1. Cosnier, S. and P. Mailley, Recent Avances in DNA Sensors. Analyst, 2008, 133(8): p. 984-991.

2. Stein, C. and J.S. Cohen, Oligodeoxynucleotides as inhibitors of gene expression: a review. Cancer Research, 1988, 48(10): p. 2659-2668.

3. Bennett, C.F. and E.E. Swayze, RNA targeting therapeutics: molecular mechanisms of antisense oligonucleotides as a therapeutic platform. Annual Review of Pharmacology and Toxicology, 2010, 50: p. 259-293.

4. Haussecker,

D.

http://rnaitherapeutics.blogspot.com/2013_01_01_archive.html.(2013) [cited 2014 12/12/2014].

5. http://www.prweb.com/releases/2006/09/prweb433853.htm. (2006) [cited $2014(12 / 12 / 2014)]$

6. Gilar, M. and E.S. Bouvier, Purification of crude DNA oligonucleotides by solid-phase extraction and reversed-phase high-performance liquid chromatography. Journal of Chromatography A, 2000, 890(1): p. 167-177.

7. Caruthers, M.H., Chemical synthesis of DNA and DNA analogs. Accounts of Chemical Research, 1991, 24(9): p. 278-284.

8. Pearson, W.H., D.A. Berry, P. Stoy, et al., Fluorous affinity purification of oligonucleotides. The Journal of Organic Chemistry, 2005, 70(18): p. 71147122. 
9. Yuan, Y., S. Fueangfung, X. Lin, et al., Synthetic 5'-phosphorylated oligodeoxynucleotide purification through catching full-length sequences by polymerization. RSC Advances, 2012, 2(7): p. 2803-2808.

10. Beller, C. and W. Bannwarth, Noncovalent Attachment of Nucleotides by Fluorous Fluorous Interactions: Application to a Simple Purification Principle for Synthetic DNA Fragments. Helvetica Chimica Acta, 2005, 88(1): p. 171-179.

11. Fang, S. and D.E. Bergstrom, Fluoride-cleavable biotinylation phosphoramidite for 5'-end-labeling and affinity purification of synthetic oligonucleotides. Nucleic Acids Research, 2003, 31(2): p. 708-715.

12. Fang, S.Y. and D.E. Bergstrom, Reversible biotinylation phosphoramidite for 5 '-end-labeling, phosphorylation, and affinity purification of synthetic Oligonucleotides. Bioconjugate Chemistry, 2003, 14(1): p. 80-85.

13. Fang, S. and D.E. Bergstrom, Reversible 5'-end biotinylation and affinity purification of synthetic RNA. Tetrahedron Letters, 2004, 45(43): p. 79877990.

14. http://www.glenresearch.com/Catalog/purification.php. [cited 2014 $12 / 15 / 2014)]$ 


\title{
Chapter 2
}

Synthetic Oligodeoxynucleotide Purification by Capping Failure Sequences with a Methacrylamide Phosphoramidite Followed by Polymerization $^{1}$

\author{
Durga Pokharel, ${ }^{\mathrm{a}}$ Yinan Yuan, ${ }^{\mathrm{b}}$ Suntara Fueangfung ${ }^{\mathrm{a}}$ and Shiyue Fang ${ }^{\mathrm{a}}$ \\ ${ }^{a}$ Department of Chemistry, Michigan Technological University, 1400 Townsend \\ Drive, Houghton, Michigan 49931, USA \\ ${ }^{\mathrm{b}}$ School of Forest Resources and Environmental Science, Michigan Technological \\ University, 1400 Townsend Drive, Houghton, Michigan 49931, USA \\ E-mail: shifang@mtu.ed
}

${ }^{1}$ The material contained in this chapter was previously published in RSC Advances, 2014, 4, 8746-8757. DOI: 10.1039/C3RA46986G

Reproduced by permission of The Royal Society of Chemistry. http://pubs.rsc.org/en/Content/ArticleLanding/2014/RA/c3ra46986g\#!divAbstract 
The idea of the project in chapter 2 was conceived by Dr. Shiyue Fang. Dr. Fang revised writing of Durga P. Pokharel. Most of the experiments in "Synthetic Oligodeoxynucleotide Purification by Capping Failure Sequences with a Methacrylamide Phosphoramidite Followed by Polymerization" were carried out by Durga P. Pokharel, except the following: Dr. Yinan Yuan did all enzyme digestion essay experiments. One compound for ODN purification was synthesized by Suntara Fueangfung.

\section{Apporved by advisor Dr. Shiyue Fang}




\begin{abstract}
Oligodeoxynucleotide (ODN) purification was achieved by capping failure sequences with a polymerizable methacrylamide phosphoramidite during automated synthesis, polymerizing the failure sequences into an acrylamide gel after cleavage and deprotection, and extraction of full-length sequences with water. The details regarding the technology including the capping efficiency of four polymerizable phosphoramidites, optimal capping time, diffusion speeds of ODN from gels with different cross-linking ratios to solution, and the efficiency of ODN extraction from gel were investigated. In addition, the technology was tested for purification of a long sequence and purification on larger scales. We also found that polymerization of failure sequences in a centrifuge tube in air did not affect purification results. Finally, we provided additional evidences that ODNs are stable under radical polymerization conditions by complete digestion of ODN followed by reversed-phase HPLC analysis of nucleosides.
\end{abstract}




\subsection{Introduction}

Applications such as total gene synthesis require the production of large numbers of short ODNs within a short time with high efficiency.[1-3] An ideal method to achieve this is through high throughput production. The high interest in using oligonucleotides (ONs) as therapeutic agents to cure human diseases generates a need for large quantities of ONs for preclinical research, clinical trial and patient use. This requires to develop technologies for large scale production of ON.[4] Currently, high throughput and large scale syntheses of $\mathrm{ON}$ are possible due to the commercialization of oligonucleotide microarray technologies, [5] high throughput automated solid phase synthesizer, [1], [6] and large scale synthesizer.[7] However, the products contain impurities, which must be removed before the applications. The impurities can be classified into three types. The first type is the truncated failure sequences generated in each synthetic cycle due to incomplete coupling of nucleoside phosphoramidite monomer. These failure sequences are capped usually with acetic anhydride to prevent them from growing in subsequent synthetic cycles and becoming the more difficult-to-remove deletion sequences. For a typical 20-mer ODN synthesis, they usually consist of 30-60\% ODN content depending on the scale of synthesis, quality of reagents and solvents, and carefulness of the synthesizer operator to minimize exposure of reagents and solvents to air.[8] Because failure sequences have very similar physical properties as the desired full-length sequences, this class of impurities is challenging to remove. The second type of impurities is small organic molecules from protecting groups. These molecules are neutral, and 
therefore are easy to remove from the negatively charged full-length sequences. The third type impurities include deletion sequences, addition sequences, and sequences resulted from depurination as well as other modifications during synthesis, cleavage and deprotection. Deletion sequences can be resulted from incomplete capping, and incomplete de-tritylation. Addition sequences can be resulted from premature detritylation. Deletion and addition sequences are most difficult to remove. Fortunately, they only exist in minute quantities in crude ODN. In ODN production, it is best to tune synthesis, cleavage and deprotection conditions to avoid the third type impurities. As a result, the major task of ODN purification usually is the removal of failure sequences from the full-length sequences.

Current methods for ODN purification include gel electrophoresis, ion exchange HPLC, trityl-on reversed-phase (RP) HPLC, RP cartridge chromatography, hydrophobic RP chromatography,[9] fluorous affinity purification,[8, 10] biotinavidin based affinity purification [11-13] and reaction-based solid phase extraction. [14] The limitations of these methods in large scale ODN purification have been discussed earlier.[15-17] For applications in high throughput purification, gel electrophoresis and all chromatographic methods are not practical or highly expensive, affinity purification methods may be expensive, and to our best knowledge, reaction-based solid phase extraction methods have not been well developed.

Recently, we communicated our preliminary results on the development of a new ODN purification technology based on catching failure sequences by polymerization. [16] Using this method, the failure sequences in crude ODN could be 
simply removed by co-polymerizing them into an insoluble polymer, and pure ODN could be obtained by simple extraction followed by $n \mathrm{BuOH}$ precipitation.[18] The method does not require any chromatography, and purification is achieved with simple manipulations such as mixing, shaking and extraction. As a result, it is potentially useful for large scale and high throughput ODN purification. In this article, we further report our detailed studies of the technology.

\subsection{Results and Discussion}

The working principle of the catching failure sequences by polymerization ODN purification method is detailed in Scheme 2.1. The entity $\mathbf{2 . 1}$ represents two of many $\mathrm{n}$-mer ODNs on solid support (usually controlled pore glass, CPG) at the end of $\mathrm{n}^{\text {th }}$ synthetic cycle, at which time the $5^{\prime}$-end DMTr (4,4'-dimethoxytrityl) group is removed (each cycle contains four steps: coupling, capping, oxidation and detritylation). Coupling 2.1 with excess phosphoramidite monomer 2.2 in the presence of an activator to generate (n+1)-mer is usually highly efficient, but it is inevitable for small amount of n-mer to fail the coupling and therefore generating failure sequences. The entity $\mathbf{2 . 3}$ represents one failure and one success sequences on solid support. Repeated coupling of $\mathbf{2 . 3}$ with $\mathbf{2 . 2}$ to complete the reaction is not a good option due to the expense of $\mathbf{2 . 2}$ and potential side reactions such as premature detritylation. However, if the failure sequences were carried on to the next synthetic cycles, they would become deletion sequences, which are highly challenging to remove during purification. As a result, they are usually capped with acetic anhydride. For using our catching failure sequences by polymerization ODN 
purification technology, however, the capping agent is replaced with the polymerizable methacrylamide phosphoramidite $\mathbf{2 . 4}$, and the resulting material is depicted with 2.5. After oxidation and detritylation steps, 2.5 becomes 2.6. The synthesis is continued and the capping failure sequences with $\mathbf{2 . 4}$ is performed in each cycle.

After cleavage and deprotection, there are mainly three entities in the crude product, the failure sequences that contain a methacrylamide group (2.7), the fulllength sequences that do not contain a methacrylamide group (2.8), and small organics from protecting groups. The crude product is subjected into radical polymerization conditions in the presence of additional polymerization monomer (e.g. $N, N$-dimethylacrylamide) and a cross-linker (e.g. $N, N^{\prime}$-methylenebis(acrylamide)).

The failure sequences are incorporated into an insoluble polymer (2.9). The fulllength sequences (2.8) and small organics remain in solution and are extracted with water. The small organics are neutral and soluble in $n \mathrm{BuOH}$, while the full-length sequences are anionic and insoluble in $n \mathrm{BuOH}$. The two are separated by precipitation of ODN 2.8 with $n \mathrm{BuOH}$ from a solution in concentrated $\mathrm{NH}_{4} \mathrm{OH}$ (Scheme 2.1). 

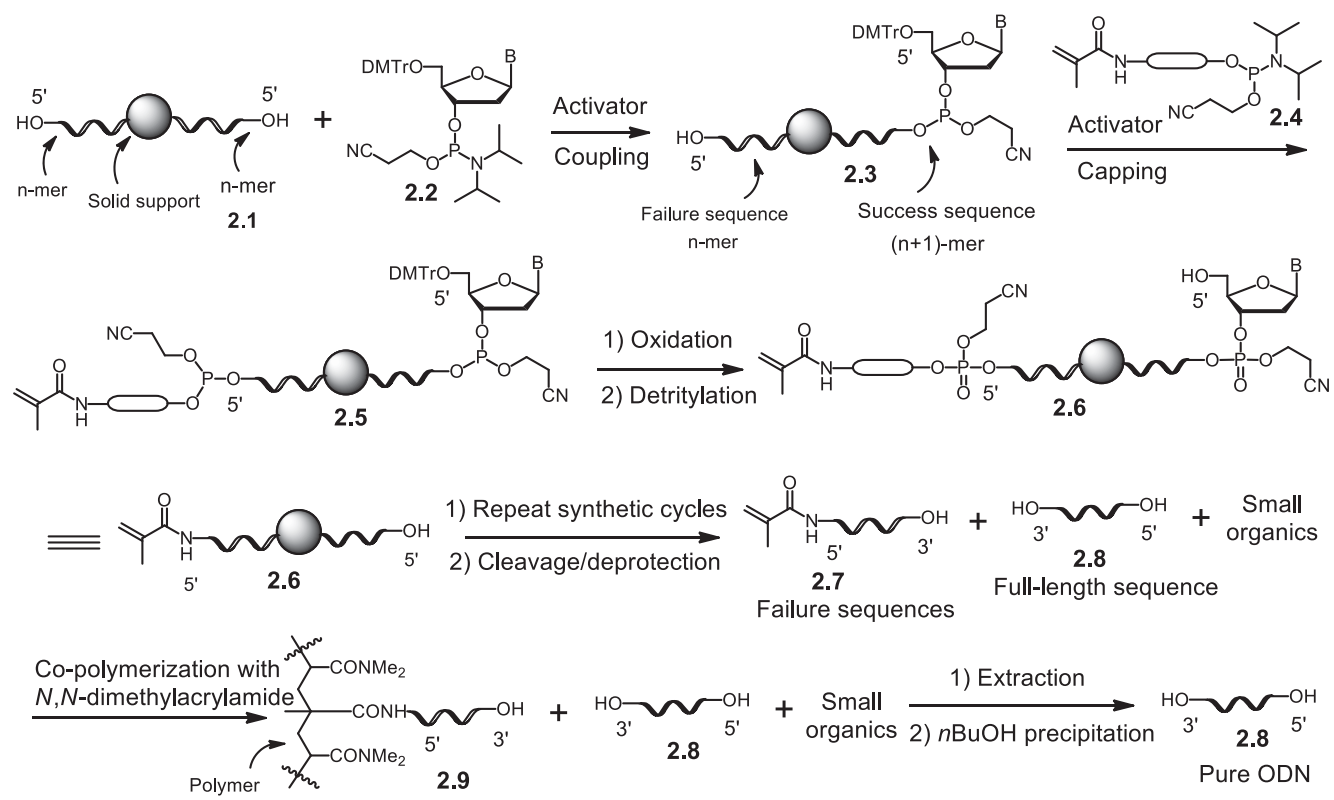

Scheme 2.1. The catching failure sequences by polymerization ODN purification method

\section{Capping Efficiency of Four Methacrylamide Phosphoramidites and Optimal}

\section{Capping Time}

Previously, we used the methacrylamide phosphoramidite $\mathbf{2 . 1 0}$ as the reagent for capping failure sequences.[16] In the present study, we synthesized three more (2.11-2.13, Figure. 2.1). The capping efficiency of them and 2.10 were evaluated. Compared with 2.10, compound 2.11 has an ether oxygen atom in the linker, which may increase its solubility in polar organic solvents such as acetonitrile and have better reaction kinetics during capping. Compound 2.12 has two ether oxygen atoms in the linker and the linker is longer. These may offer benefits for reaction kinetics in both capping and polymerization steps. Compound $\mathbf{2 . 1 3}$ has an even longer linker, and in the linker, there is an amide bond. It was expected that the compound exist as a 
solid at room temperature and therefore its purification and storage be more convenient. Compound 2.11 was simply synthesized from amino alcohol 2.14 in two steps in excellent yields (Scheme 2.2). The intermediate compound $\mathbf{2 . 1 5}$ is known, [19] but we used a different procedure for its preparation (see experimental section). Capping phosphoramidites $\mathbf{2 . 1 2 - 2 . 1 3}$ were prepared from 2.16, [20] and 2.18, [21] respectively using the same procedures for preparing 2.11. The intermediate compound 2.17 was known, [22] and 2.19 is new and was fully characterized.<smiles>C=C(C)C(=O)NCCCCCCOP(OCCC#N)N(C(C)C)C(C)C</smiles>

2.10<smiles>[Z10]#CCNC(=O)C(=C)C</smiles>

2.11<smiles>[Z12]C(C)C(=O)NCCOCCOCCOP(OCCC#N)N(C(C)C)C(C)C</smiles><smiles>[Z13]CCOCCOCCNC(=O)CCCOP(OCCC#N)N(C(C)C)C(C)C</smiles>

Figure 2.1. Structures of polymerizable capping phosphoramidites.

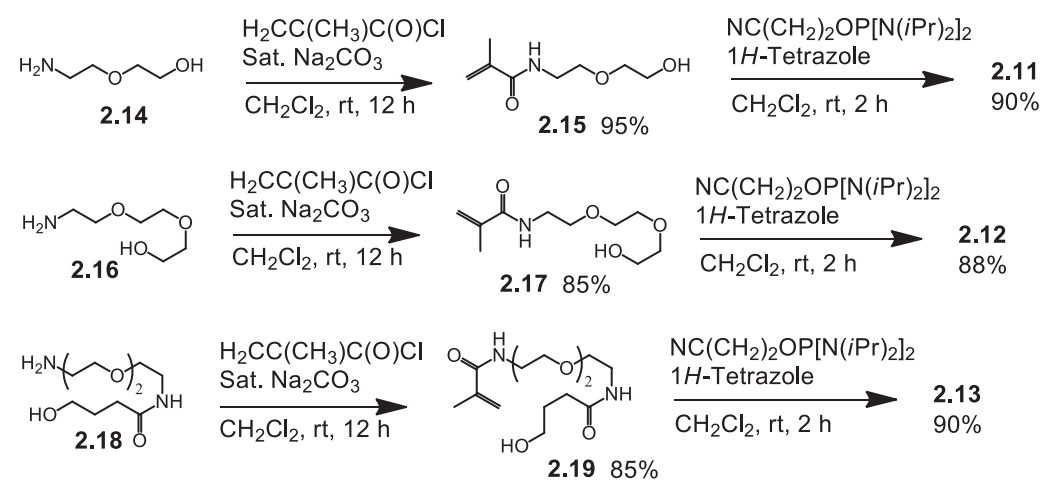

Scheme 2.2. Synthesis of polymerizable capping phosphoramidites. 
In our initial studies, during automated ODN synthesis, capping failure sequences was performed after the oxidation step that oxidizes the newly formed phosphite triester bonds in the success sequences.[16] As a result, after capping, another oxidation step was needed to oxidize failure sequences. In the present study, we planned to simplify the process by omitting the first oxidation step and to oxidize the success and failure sequences in one single step. In addition, reduction of capping delivery time and the concentration of capping solutions were also planned. To test feasibility, the 20-mer ODN 2.20 was synthesized using a $0.15 \mathrm{M}$ acetonitrile solution of 2.11 for capping. After the coupling steps, without oxidation, the capping solution was delivered to the synthesis column along with the activator $1 H$-tetrazole.

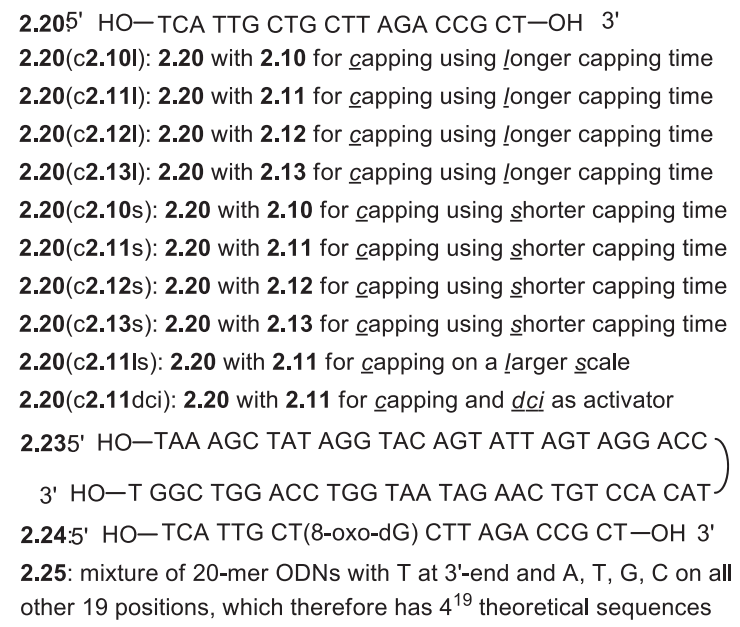

Figure 2.2. Labelling of ODNs 
The delivery time and manner were about the same as in the coupling step except that the reagents were delivered for two more times, and after each delivery a waiting step was added. More specifically, the reagents were delivered to the synthesis column for a total of four times $(2.0$ seconds, 1.5 seconds $\times 3)$. After each delivery the reagents remained in the column for 90 seconds (Cycle 1 in supporting information). These capping steps consumed less capping phosphoramidite compared with those used in our initial studies, which were 2.5 seconds, 1.5 seconds $\times 3$ with $0.2 \mathrm{M}$ solution.[16] After the capping steps, the column was washed with acetonitrile, and the synthesis was allowed to proceed as normal.

The final detritylation, cleavage and deprotection were also carried out under commonly used conditions. The crude product, which was labeled as $2.20(\mathrm{c} 2.111)$ (ODN labeling is summarized in Figure 2.2), was analyzed with RP HPLC (trace a, Figure 2.3). 

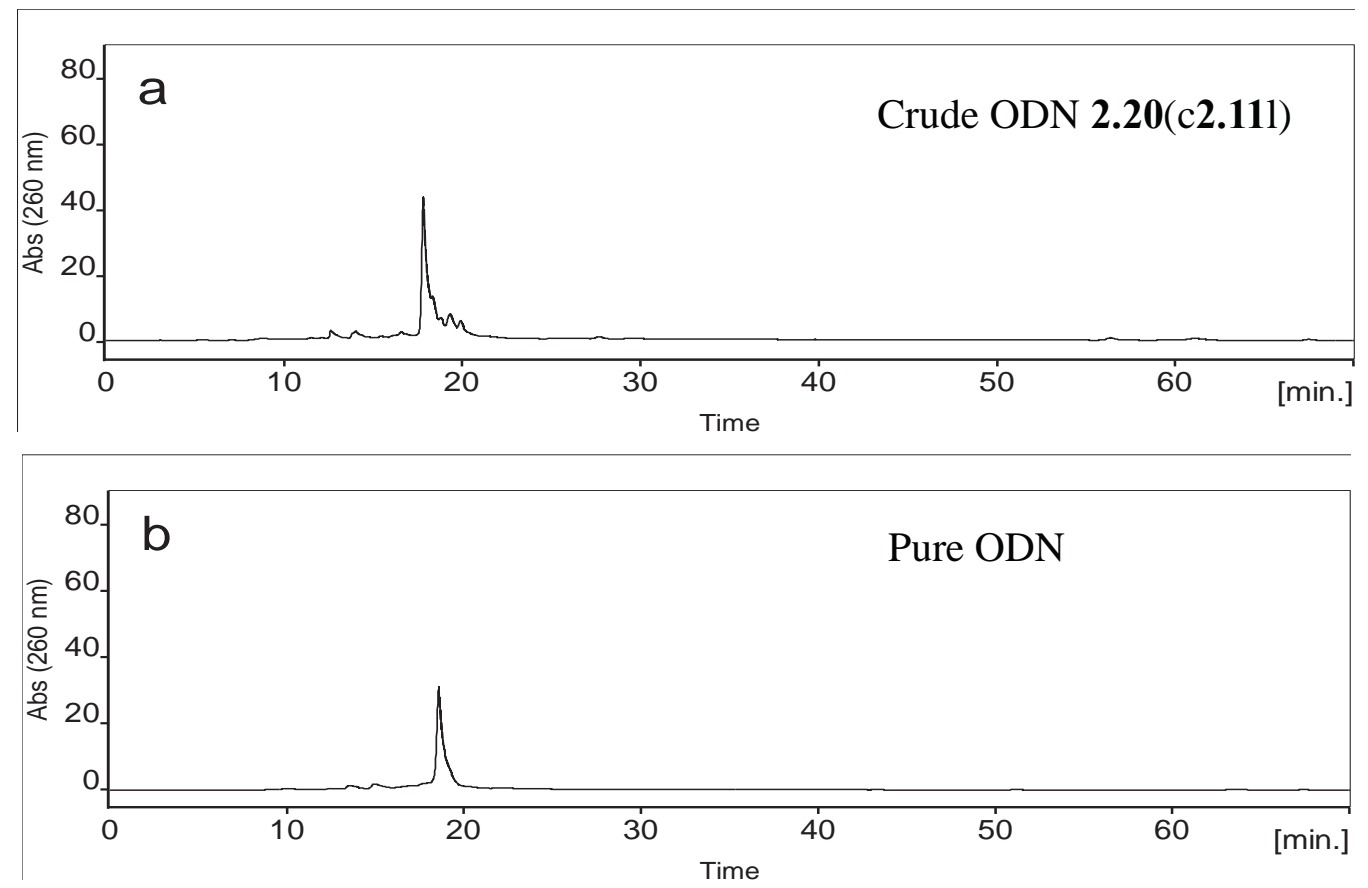

Figure 2.3. RP HPLC profiles of ODN 2.20(c2.111). (b) Crude ODN. (b) ODN purified with catching by polymerization

The remaining crude $\mathbf{2 . 2 0}(\mathbf{c 2 . 1 1 1})$ was subjected to radical acrylamide polymerization conditions widely used for the preparation of gels in electrophoresis experiments (Scheme 2.3). 

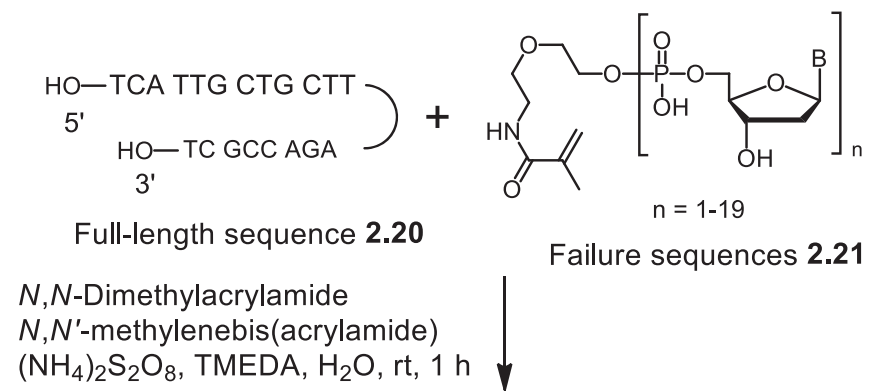

Failure sequences $\mathbf{2 . 2 1}$

$N, N^{\prime}$-methylenebis(acrylamide)

$\left(\mathrm{NH}_{4}\right)_{2} \mathrm{~S}_{2} \mathrm{O}_{8}, \mathrm{TMEDA}, \mathrm{H}_{2} \mathrm{O}, \mathrm{rt}, 1 \mathrm{~h}$

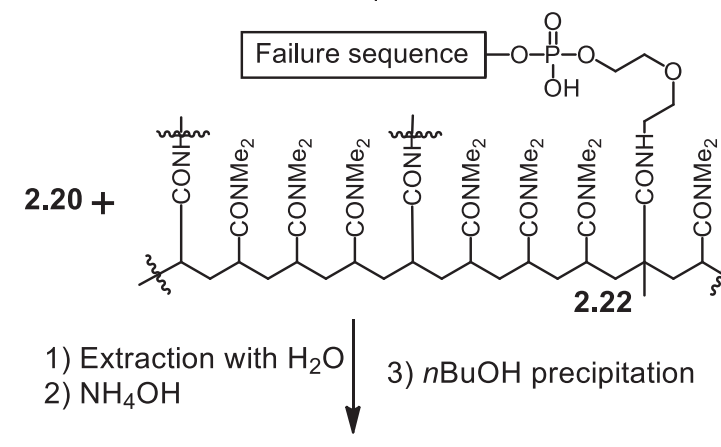

Pure 2.20

Scheme 2.3. Purification of ODN 2.20 by polymerization of failure sequences

Briefly, the crude ODN, which mainly contained the full-length sequences 2.20, the failure sequences $\mathbf{2 . 2 1}$ and small organic molecules from protecting groups, was mixed with a pre- prepared polymerization solution containing the polymerization monomer $N, N$-dimethylacrylamide and the cross-linker $N, N^{\prime}$ methylenebis(acrylamide). Polymerization was then initiated under a nitrogen atmosphere with ammonium persulfate and $N, N, N^{\prime}, N^{\prime}$-tetramethylethylenediamine (TMEDA).

The failure sequences were copolymerized into the acrylamide gel $\mathbf{2 . 2 2}$. The fulllength sequences (2.20) and small organics remained in solution, and were extracted 
with water. After evaporation of volatiles and heating with concentrated $\mathrm{NH}_{4} \mathrm{OH}$ briefly, the ODN was precipitated with $n \mathrm{BuOH}$.[16] The purified 2.20(c2.111) was analyzed with RP HPLC (trace b, Figure. 2.3) and the purity was found to be $93 \%$. The recovery yield of the purification process was estimated to be $93 \%$ by dividing the area of the peak at 19 minutes in trace $b$ by that in trace a in Figure 2.3. The results indicate that the new agent $\mathbf{2 . 1 1}$ with the simplified capping procedure is capable of capping failure sequences completely. We also tested the capping agents 2.10, 2.12 and 2.13 under the same new ODN synthesis and catching by polymerization purification conditions. All of them were found equally suitable for the application. The RP HPLC profiles for ODNs 2.20(c2.101), 2.20(c2.121) and 2.20(c2.131) are shown in supporting information. Their recovery yields and purity are shown in Table 2.1 .

In order to see if capping time could be further shortened and at the same time to distinguish the efficiency of the four capping reagents, the above ODN synthesis procedure was repeated with the capping conditions being changed from phosphoramidite and activator delivering time of 2.0 seconds, 1.5 seconds $\times 3$, and waiting time of 90 seconds after each delivery to delivering time of 2.0 seconds, 1.5 seconds $\times 2$, and waiting time of 30 seconds (Cycle 2 in supporting information). After cleavage and deprotection, the four crude samples 2.20(c2.11s), 2.20(c2.10s), 2.20(c2.12s) and 2.20(c2.13s) were purified using the same procedure as described above. The purified ODNs were analyzed with RP HPLC, and good purity and recovery yields were found (Table 2.1). Because the delivery time of capping reagents was almost the same as that of nucleoside phosphoramidite monomers in 
typical coupling steps (2.0 seconds, 1.5 seconds), further shortening of capping time was not pursued. All the four capping phosphoramidites (2.10-2.13) performed equally well, and therefore any of them could be used for the catching by polymerization technology. However, compounds 2.12-2.13 are relatively more expensive to make. Compound $\mathbf{2 . 1 3}$ did not exist as a solid or foam at room temperature as we hoped, and moreover, it was found more prone to oxidation during purification. As a result, compounds 2.10-2.11 are preferred capping reagents for the catching failure sequences by polymerization purification technology.

Table 2.1 ODN purity and recovery yield ${ }^{a}$

\begin{tabular}{lcc}
\hline ODN & Purity & Recovery yield \\
\hline $\mathbf{2 . 2 0}(\mathrm{c} \mathbf{2 . 1 0 1})$ & $89 \%$ & $92 \%$ \\
$\mathbf{2 . 2 0}(\mathrm{c} 2.111)$ & $93 \%$ & $93 \%$ \\
$\mathbf{2 . 2 0}(\mathrm{c} 2.121)$ & $99 \%$ & $72 \%$ \\
$\mathbf{2 . 2 0}(\mathrm{c} 2.131)$ & $100 \%$ & $81 \%$ \\
$\mathbf{2 . 2 0}(\mathrm{c} 2.10 \mathrm{~s})$ & $100 \%$ & $82 \%$ \\
$\mathbf{2 . 2 0}(\mathrm{c} \mathbf{2 . 1 1} \mathrm{s})$ & $100 \%$ & $86 \%$ \\
$\mathbf{2 . 2 0}(\mathrm{c} \mathbf{2 . 1 2})$ & $98 \%$ & $95 \%$ \\
$\mathbf{2 . 2 0}(\mathrm{c} 2.13 \mathrm{~s})$ & $94 \%$ & $70 \%$ \\
$\mathbf{2 . 2 3}$ & $92 \%$ & $67 \%$ \\
$\mathbf{2 . 2 0}(\mathrm{c} 2.111 \mathrm{~s})$ & $94 \%$ & $73 \%$ \\
$\mathbf{2 . 2 0}(\mathrm{c} 2.11 \mathrm{dci})$ & $100 \%$ & $88 \%$ \\
\hline
\end{tabular}

${ }^{a}$ Purity was calculated from the areas of ODN and impurity peaks in HPLC profile. Yield was calculated by dividing the area of ODN peak in HPLC profile of purified sample by that in the profile of crude sample. 


\section{Diffusion Speed of ODN From Gel and Extraction Efficiency}

The diffusion speed of ODN from the acrylamide gel to solution is an important factor to determine the efficiency of ODN extraction. The purification technology is most useful if the diffusion speed is high and ODN can be extracted with high efficiency and recovery yield. Our common sense was that gels with higher crosslinking ratio [the mole ratio of $N, N^{\prime}$-methylenebis(acrylamide) over $N, N$ dimethylacrylamide] would result in lower diffusion speed and extraction efficiency. Gels with lower cross-linking ratio were expected to give better results. However, if the cross-linking ratio is too low, the gel may be too soft and therefore difficult to handle. We were interested to find a compromised point where the most common 20mer ODNs could be extracted with acceptable efficiency while the gel is hard enough to be easily handled. From experiments on ODN purification with polyacrylamide gel electrophoresis, it is known that gels with a cross-linking ratio of 1:25 are reasonably hard and 20-mer ODNs can be extracted from them with acceptable efficiency and yields. We therefore decided to start our experiments with this cross-linking ratio. 
Table 2.2 Monitoring ODN diffusion from gels with different cross-linking ratios to supernatant $^{a}$

\begin{tabular}{|c|c|c|c|c|c|c|c|c|c|c|c|}
\hline Entry & $\begin{array}{l}\text { Time } \\
(\min )\end{array}$ & $\begin{array}{l}\text { ODN Con. } \\
(\mathrm{ng} / \mu \mathrm{L})^{b}\end{array}$ & $\begin{array}{c}\% \text { of } \\
\mathrm{ODN}^{b, g}\end{array}$ & $\begin{array}{l}\text { ODN Con. } \\
(\mathrm{ng} / \mu \mathrm{L})^{c}\end{array}$ & $\begin{array}{c}\% \text { of } \\
\mathrm{ODN}^{c, g}\end{array}$ & $\begin{array}{l}\text { ODN Con. } \\
(\mathrm{ng} / \mu \mathrm{L})^{d}\end{array}$ & $\begin{array}{c}\% \text { of } \\
\mathrm{ODN}^{d, g}\end{array}$ & $\begin{array}{l}\text { ODN Con. } \\
(\mathrm{ng} / \mu \mathrm{L})^{e}\end{array}$ & $\begin{array}{c}\% \text { of } \\
\mathrm{ODN}^{e, g}\end{array}$ & $\begin{array}{l}\text { ODN Con. } \\
(\mathrm{ng} / \mu \mathrm{L})^{f}\end{array}$ & $\begin{array}{c}\% \text { of } \\
\text { ODN }^{f g}\end{array}$ \\
\hline 1 & 10 & 47.7 & 66.0 & 29.9 & 47.5 & 32.7 & 45.0 & 27.1 & 39.9 & 25.8 & 39.3 \\
\hline 2 & 20 & 53.3 & 73.7 & 37.8 & 60.1 & 40.9 & 56.3 & 36.0 & 53.0 & 34.7 & 52.9 \\
\hline 3 & 30 & 54.7 & 75.7 & 43.4 & 69.0 & 49.5 & 68.2 & 42.0 & 61.9 & 37.7 & 57.5 \\
\hline 4 & 40 & 58.6 & 81.1 & 48.6 & 77.3 & 53.4 & 73.6 & 46.9 & 69.1 & 42.8 & 65.2 \\
\hline 5 & 50 & 64.6 & 89.3 & 50.9 & 80.9 & 56.7 & 78.1 & 50.5 & 74.4 & 47.0 & 71.7 \\
\hline 6 & 60 & 65.1 & 90.0 & 54.6 & 86.8 & 59.2 & 81.5 & 53.3 & 78.5 & 50.0 & 76.2 \\
\hline 7 & 70 & 66.4 & 91.8 & 56.0 & 89.0 & 64.2 & 88.4 & 56.1 & 82.6 & 53.3 & 81.3 \\
\hline 8 & 80 & 67.6 & 93.5 & 58.0 & 92.2 & 64.6 & 89.0 & 59.7 & 87.9 & 56.0 & 85.4 \\
\hline 9 & 90 & 68.6 & 94.9 & 59.6 & 94.8 & 67.2 & 92.6 & 59.9 & 88.2 & 58.6 & 89.3 \\
\hline 10 & 100 & 70.9 & 98.1 & 61.2 & 97.3 & 68.0 & 93.7 & 64.0 & 94.3 & 59.8 & 91.2 \\
\hline 11 & 110 & 71.7 & 99.2 & 62.0 & 98.6 & 70.6 & 97.3 & 66.2 & 97.5 & 65.4 & 99.7 \\
\hline 12 & 120 & 72.3 & 100 & 62.9 & 100 & 72.6 & 100 & 67.9 & 100 & 65.6 & 100 \\
\hline
\end{tabular}

${ }^{a}$ Crude ODN containing 2.20 and 2.21 was copolymerized into gel. Water was added to extract 2.20. The concentration of 2.20 in the supernatant was monitored with UV over a two-hour period.

${ }^{b-f}$ In supernatants of gel with cross-linking ratio of 1:2, 1:7, 1:15, 1:25, 1:50, respectively.

${ }^{g}$ Calculated by dividing ODN concentration at a specific time by that after $120 \mathrm{~min}$.

A portion of crude ODN 2.20(c2.11s), which contained 2.21, was subjected to polymerization using a polymerization solution that had a mole ratio of cross-linker over monomer of 1:25 (see details in Experimental section). Water was added to the gel, and small aliquots of supernatant were taken out every $10 \mathrm{~min}$ and the concentrations of ODN were determined with UV measurement. As shown in Table 2.2, after $10 \mathrm{~min}$, the concentration of ODN in the supernatant had reached about $40 \%$ (entry 1 ) of the value at $2 \mathrm{~h}$, at which time the diffusion was close to 
equilibrium. For the concentration to reach $90 \%$ of equilibrium value, about $90 \mathrm{~min}$ were required (entry 9). To find out how many times were needed to extract all or most ODN from gel, after $2 \mathrm{~h}$, the supernatant was removed completely (entry 12, Table 2.2 and entry 1, Table 2.3). To the gel, water was added again, and after $2 \mathrm{~h}$, the supernatant was removed and its concentration was determined with UV measurement (entry 2, Table 2.3). The extraction and UV measurement were repeated for three additional times. As shown in Table 3, after two extractions, only about 3\% extractable ODN remained in the gel (entry 3). After three extractions, the value lowered to $0.4 \%$ (entry 4). The results in Tables 2.2 and 2.3 indicate that ODN 2.20(c2.11s) can be efficiently extracted from gel that has a cross-linking ratio of 1:25 with water for three times in a total of $6 \mathrm{~h}$.

Table 2.3 Extraction efficiency of ODN from gel ${ }^{a}$

\begin{tabular}{|c|c|c|c|c|c|c|c|c|c|c|c|}
\hline Entry & $\begin{array}{l}\text { Time } \\
\text { (h) }\end{array}$ & $\begin{array}{l}\text { ODN Con. } \\
(\mathrm{ng} / \mu \mathrm{L})^{b}\end{array}$ & $\begin{array}{c}\% \text { of } \\
\mathrm{ODN}^{b, g}\end{array}$ & $\begin{array}{l}\text { ODN Con. } \\
(\mathrm{ng} / \mu \mathrm{L})^{c}\end{array}$ & $\begin{array}{c}\% \text { of } \\
\mathrm{ODN}^{c, g}\end{array}$ & $\begin{array}{c}\text { ODN Con. } \\
(\mathrm{ng} / \mu \mathrm{L})^{d}\end{array}$ & $\begin{array}{c}\% \text { of } \\
\mathrm{ODN}^{d, g}\end{array}$ & $\begin{array}{l}\text { ODN Con. } \\
(\mathrm{ng} / \mu \mathrm{L})^{e}\end{array}$ & $\begin{array}{c}\% \text { of } \\
\text { ODN }^{e, g}\end{array}$ & $\begin{array}{l}\text { ODN Con. } \\
(\mathrm{ng} / \mu \mathrm{L})^{f}\end{array}$ & $\begin{array}{c}\% \text { of } \\
\text { ODN } N^{f, g}\end{array}$ \\
\hline 2 & 4 & 9.5 & 11.2 & 10.3 & 13.5 & 18.1 & 19.3 & 23.5 & 24.8 & 22.3 & 21.5 \\
\hline 4 & 8 & 0.0 & 0.00 & 0.0 & 0.0 & 0.0 & 0.0 & 0.4 & 0.4 & 2.1 & 2.0 \\
\hline 5 & 10 & 0.0 & 0.00 & 0.0 & 0.0 & 0.0 & 0.0 & 0.3 & 0.3 & 0.4 & 0.4 \\
\hline
\end{tabular}

\footnotetext{
${ }^{a}$ The experiments in Table 2.1 were continued by removing the supernatant after $2 \mathrm{~h}$. Water was added to the gels again, and supernatants were removed after $2 \mathrm{~h}$. The extractions were repeated every $2 \mathrm{~h}$ for three additional times.

${ }^{b-f}$ In supernatants of gel with cross-linking ratio of $1: 2,1: 7,1: 15,1: 25,1: 50$, respectively.

${ }^{g}$ Calculated by dividing ODN concentration in each extract by that of the total amount of ODN extracted from the gel in the same volume of water.
} 
To find out if a gel with lower cross-linking ratio could offer higher diffusion speed and better extraction efficiency, the above experiments were repeated with the exception of using a polymerization solution that had a cross-linking ratio of 1:50. As shown in Tables 2.2 and 2.3, the diffusion speed and extraction efficiency were not better, which was contrary to our prediction. Instead, the data showed a slightly slower diffusion speed and lower extraction efficiency although the differences were small (e.g. $39.3 \%$ vs. $39.9 \%$ at 10 min entry 1 , and $91.2 \%$ vs. $94.3 \%$ at 100 min entry 10, Table 2.2). Because gels with a higher cross-linking ratio gave slightly better results, we repeated the experiments with a cross-linking ratio of 1:15 (Tables 2.2 and 2.3). According to the trend, the diffusion speed and extraction efficiency should become better, which was indeed the case although the difference was small again. For example, within $10 \mathrm{~min}$, the concentration of ODN in the supernatant had reached about $45 \%$ of equilibrium value (entry 1). For the concentration to reach $90 \%$ of equilibrium value, about 80 min were required (entry 8). For extraction efficiency, after three extractions, no more ODN was detectable in additional extracts (entry 4, Table 2.3). We then further increased the cross-linking ratio to 1:7. The trends of diffusion speed and extraction efficiency changes were the same. Within $10 \mathrm{~min}$, the concentration of ODN in the supernatant had reached $48 \%$ of equilibrium value (entry 1). For the concentration to reach $90 \%$ of equilibrium value, about 70 min were needed (entry 7). When the cross-linking ratio was increased to 1:2, within 10 min, the concentration reached $66 \%$ (entry 1), and the time for reaching $90 \%$ was reduced to 60 min (entry 6). These results consistently show that the diffusion speed and ODN extraction efficiency increase with increased cross-linking ratio of gel. This is 42 
significant for the technology because gels with higher cross-linking ratios are harder and therefore easier to handle.

It was quite surprising to find that the ODN diffusion speed and extraction efficiency increase with cross-linking ratio. We had expected the contrary because gels with higher cross-linking ratio have smaller pores and ODN molecules would be more difficult to travel through them. Our explanation of the observed results is that the ODN molecules were not totally in the gel even though this seemed impossible because the gel with other contents including water and ODN looked like homogenous. Instead, when the polymers grew, ODN molecules were expelled or partially expelled out from the polymer matrix. With lower cross-linking ratio when the pores in polymer were larger, a higher portion of ODN molecules was in the polymer matrix, or all ODN molecules were not in the polymer matrix, but they could diffuse into it with relatively high rates. These resulted in seemingly lower diffusion speeds of ODN from gels to solution and lower extraction efficiency. With higher cross-linking ratio when the pores in the polymer were smaller, a lower portion of ODN molecules was in the polymer matrix, or all ODN molecules were not in the polymer matrix, and they could only diffuse into it with low rates. These resulted in seemingly higher diffusion speeds of ODN from gels to solution and higher extraction efficiency.

\section{Long Sequence Purification}

For long sequence ODN purification, the catching failure sequences by polymerization method requires significant amount of polymerizable capping phosphoramidite, which is more expensive than acetic anhydride or phenoxyacetic 
anhydride capping agents used in catching full-length sequences by polymerization method.[15] We therefore recommended the catching full-length sequences by polymerization method for long sequence purification.[16, 23] However, it is still interesting to test the suitability of the catching failure sequences method for ODN purification because it may be more convenient in certain applications such as high throughput purification. The 61-mer ODN 2.23, which is a portion of the HIV protease gene, was selected as the target. The synthesis was carried out on a $0.2 \mu \mathrm{mol}$ scale using the procedure with shortened capping time. Polymerizable phosphoramidite 2.11 was used as the capping agent. In the coupling step, after finishing phosphoramidite and activator delivery, an additional waiting of 30 seconds was added to increase yields (Cycle 2 in supporting information with additional waiting after coupling). The crude ODN was purified by catching failure sequences by polymerization as we did for shorter sequences. RP HPLC analysis showed that the ODN had good purity and recovery yield (Table 2.1 and supporting information). The identity of purified ODN was confirmed with ESI-MS (supporting information).

\section{Purification at Larger Scales}

After investigation of the effect of several additional factors such as capping agent storage time, radical scavenger, harsher deprotecting conditions, lower concentration of capping solution, polymerization in air (see supporting information), we decided to test the technology for larger scale purification. For this goal, ODN 2.20 was synthesized on a $1 \mu \mathrm{mol}$ scale. The synthesis conditions were only slightly different from those using shortened capping time described earlier, which was used for $0.2 \mu \mathrm{mol}$ synthesis. The standard $1 \mu \mathrm{mol}$ synthesis cycle provided by 
manufacturer of the synthesizer was modified. The steps for capping with acetic anhydride were replaced with those for capping with polymerizable phosphoramidite. The latter was achieved by delivering the solution of $\mathbf{2 . 1 1}$ in acetonitrile and activator to the synthesis column in the same manner as in the coupling steps except that an additional delivery was added and a waiting of 30 seconds was added after each delivery (Cycle 3 in supporting information). At the end of synthesis, DMTr group was removed, and cleavage and deprotection were performed. The crude ODN was purified by catching failure sequences by polymerization using the same procedure described earlier. The only differences were that the $1 \mu \mathrm{mol}$ ODN was purified in one batch, the volume of the polymerization solution was increased to 10 times of that used in the general $0.05 \mu \mathrm{mol}$ purification procedure, and the volume of extraction water was slightly larger. The ODN, which is labeled as $\mathbf{2 . 2 0}(\mathrm{c} 2.11 \mathrm{ls})$, was found to have good purity and recovery yield with RP HPLC (Table 2.1 and supporting information). It is remarkable that even though the purification scale was increased from $0.05 \mu \mathrm{mol}$ to $1 \mu \mathrm{mol}$, which was 20 times, increasing the volume of polymerization solution to 10 times was sufficient to catch all the failure sequences.

We further performed three $1 \mu$ mol syntheses simutaneously under conditions that are more close to actual large scale ODN production. Specifically, $1 H$-tetrazole is considered as an explosive and not recommended for large scale synthesis. Therefore, 4,5-dicyanoimidazole (DCI) was used as activator. To save phosphoramidite monomers, their concentration was lowered from $0.1 \mathrm{M}$ to $0.05 \mathrm{M}$. A $0.1 \mathrm{M}$ solution of 2.11 in acetonitrile was used for capping. With these modifications, ODN 2.20 was synthesized under otherwise the same conditions for the synthesis of $\mathbf{2 . 2 0}(\mathrm{c} 2.111 \mathrm{~s})$. 
According to trityl assay and RP HPLC analysis of crude ODN, the synthesis yields were not significantly different from synthesis under conditions without the above modifications. The $3 \mu \mathrm{mol}$ ODN was purified in one batch under the same conditions described for purification of $\mathbf{2 . 2 0}$ (c2.11ls) and is labeled as $\mathbf{2 . 2 0}$ (c2.11dci). RP HPLC analysis indicated that the ODN was highly pure and had excellent recovery yield (Table 2.1 and supporting information).

\section{ODN Stability Under Radical Polymerization Conditions}

A concern on the catching by polymerization purification technologies, which involves copolymerization of ODN into polymer under free radical conditions, is the potential damage of ODN under such conditions. Polymerization of ODN into polyacrylamide gels under radical conditions is known in the literature.[24-26] 

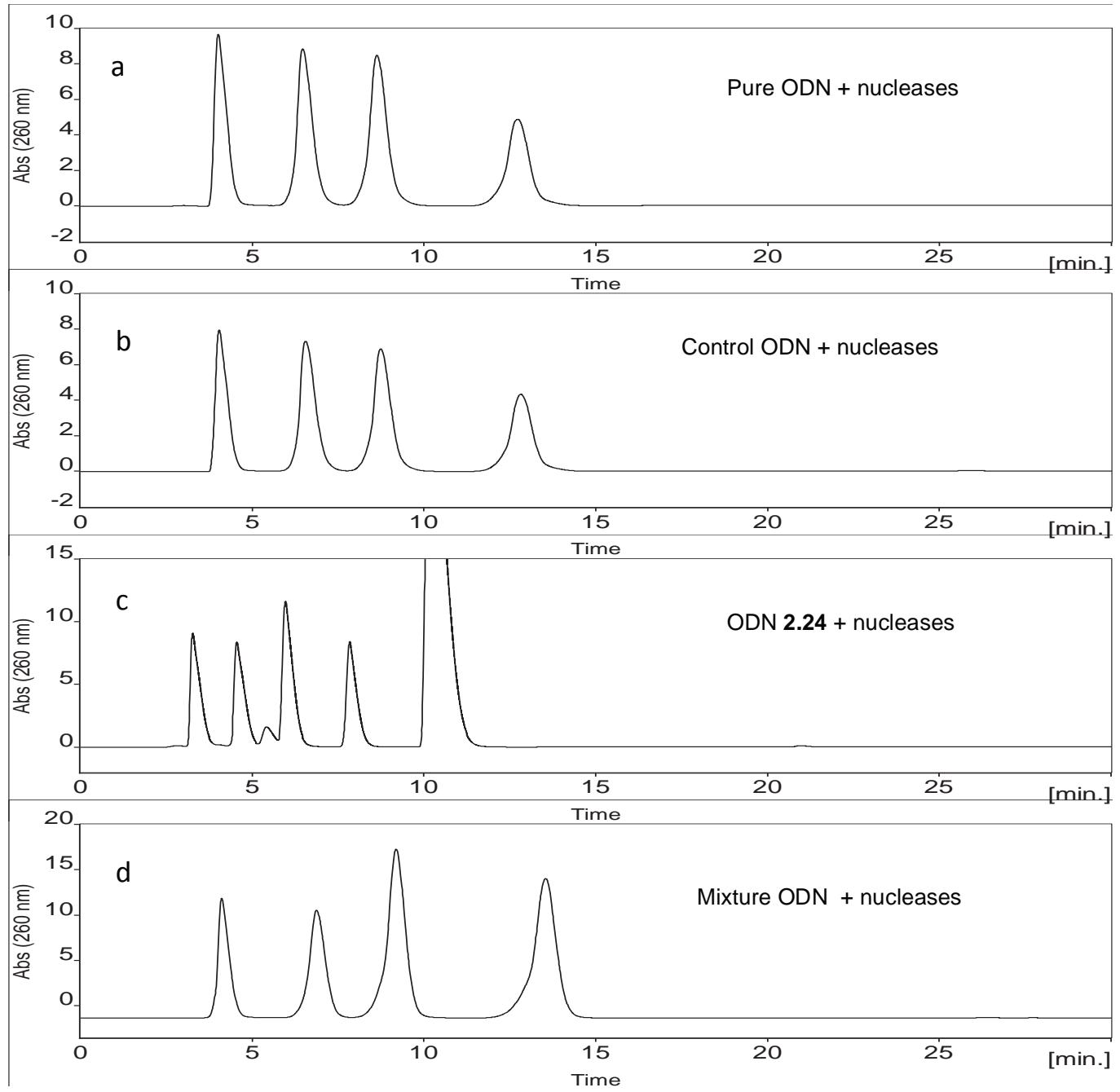

Figure. 2.4 RP HPLC profiles of nucleosides from ODN enzymatic digestion essay. (a) Nucleosides from ODN 2.20(c2.11s), which was purified with the catching by polymerization technique. (b) Nucleosides from authentic ODN 2.20. (c) Nucleosides from ODN 2.24. (d) Nucleosides from the mixture ODN 2.25. The peaks in a-b and d correspond to $\mathrm{dC}, \mathrm{dG}, \mathrm{dT}$ and $\mathrm{dA}$, respectively. Those in c correspond to $\mathrm{dC}, \mathrm{dG}, 8$ oxo-dG, dT and $\mathrm{dA}$, and 2-mercaptoethanol, respectively. The inconsistency of retention times in c with those in a-b and $d$ is caused by the use of a different column. 
However, to our best knowledge, detailed study of ODN stability in the presence of free radicals has not been reported. Certainly, RP HPLC analysis of ODNs purified with the catching by polymerization technologies by comparing with authentic ODN strongly supports that ODNs can survive such conditions. Another evidence of stability comes from mass spectrometry, which consistently gave molecular mass consistent with calculated values.[15-17] Further, we also subjected the nucleosides guanosine, adenosine, thymidine and cytidine to free radical acrylamide polymerization conditions, extracted them from the polymer, and analyzed them with RP HPLC. No damaged nucleoside was detected. Among the four nucleosides, guanosine oxidation is more common, and the oxidation product is 8-oxo-guanosine. We compared the guanosine from the polymer with authentic 8-oxo-guanosine by coinjection into RP HPLC, the two were found to have different retention times, and no 8-oxo-guanosine was detected in the sample from polymer.[15] However, concerns on ODN stability may still remain because this is so important an issue for purification, especially for ODN drug purification. For RP HPLC analysis, if only one of the 20 bases of a 20-mer ODN is damaged, and the physical properties of the damaged nucleobase are not changed significantly, the analysis may not be able to differentiate damaged ODN from authentic ODN. For mass spectrometry analysis, if only a minute portion of ODN is damaged, the method may not be sensitive enough for detection. For the experiments to test stability of nucleosides under radical conditions, the reactivity of nucleosides in an ODN may not be the same as individual 
nucleosides. As a result, all the methods we used to support ODN stability under radical conditions are not conclusive.

To obtain additional evidences on the stability of ODN under radical acrylamide polymerization conditions, we used ion exchange HPLC to analyze one of our samples purified with the catching failure sequences by polymerization process. It was expected that ion exchange HPLC have different resolution on potentially damaged ODNs than RP HPLC. To our satisfaction, we still did not found any damaged ODN, and only one peak was observed (supporting information). We also tried to find any impurity using RP HPLC by UV detecting at $210 \mathrm{~nm}$ instead of the usually used $260 \mathrm{~nm}$; again, only one peak was observed (supporting information). Further, we performed an ODN enzymatic digestion essay to detect any damaged nucleosides.[27] A portion of the ODN sample 2.20(c2.11s) purified with the catching failure sequences by polymerization method was digested with Snake Venom Phosphodiesterase (SVP) into nucleotides, which were dephosphorylated to give nucleosides by Bacterial Alkaline Phosphatase (BAP). The neutral nucleosides were then separated from the highly polar species including inorganic salts and enzymes in the digestion mixture by precipitation of the latter materials by ethanol from a sodium acetate buffer. The nucleosides were soluble in ethanol and remained in the supernatant. The polar species were not soluble and were precipitated. The nucleosides were analyzed with RP HPLC. As shown in Figure 2.4, only four peaks, which correspond to the four natural nucleosides, were observed (trace a). No additional peaks that correspond to any damaged nucleosides were detected. An authentic ODN 2.20, which was from a commercial source and was purified with 
preparative RP HPLC, was also subjected into the same digestion process. The resulting HPLC profile of nucleosides (trace b) was exactly the same as that from 2.20(c2.21s). Another ODN (2.24), which is $\mathbf{2 . 2 0}$ with one dG being replaced with 8oxo-dG (Figure 2.2), was synthesized and purified by DMTr-on preparative RP HPLC (see supporting information for crude and pure HPLC profiles and MALDITOF MS), and subjected to digestion.[28] The HPLC profile (trace c) showed five peaks besides a peak from 2-mercaptoethanol indicating that the essay could detect potentially damaged nucleosides. The relative retention times of the nucleosides including 8-oxo-dG were consistent with literature values.[27, 28] To address the concern that ODN damage may be dependent on the secondary structures resulted from different sequences, a complex mixture of 20-mer ODN (2.25), which contained $4^{19}$, theoretical sequences, were synthesized using a mixture of the four nucleoside phosphoramidite monomers. The synthesis procedure with a shortened capping time was used (Cycle 2 in supporting information), and the failure sequences were capped with methacrylamide phosphoramidite 2.11. The crude ODN was subjected to the general catching failure sequences by polymerization process (supporting information). The resulting mixture of full-length sequences was subjected to the same SVP digestion, BAP dephosphorylation, and RP HPLC analysis process (trace d, Figure 2.4). Only four peaks were observed. To confirm that the peaks were from ODN and not from SVP and BAP, the essay process was repeated without using any ODN. No nucleoside peaks were observed (supporting information). The four peaks in traces a-b and d correspond to $\mathrm{dC}, \mathrm{dG}, \mathrm{dT}$ and $\mathrm{dA}$, respectively. The areas of them are consistent with calculated values (supporting information). [27] These results 50 
provide strong evidence that ODNs with different sequences are stable under the radical acrylamide polymerization conditions. Further analysis to detect any minute quantities of potentially damaged ODNs under the radical conditions is beyond the scope of this study. Future directions would be to use the purified ODNs in molecular biology experiments.

\subsection{Experimental}

\section{General Methods}

Reagents and solvents commercially available were used as received unless otherwise noted. $\mathrm{CH}_{2} \mathrm{Cl}_{2}$ was distilled over $\mathrm{CaH}_{2}$ under a nitrogen atmosphere. Reactions were carried out under nitrogen in oven-dried glassware. Thin layer chromatography (TLC) was performed using TLC plates with silica gel 60F-254 over glass support, $0.25 \mu \mathrm{m}$ thickness. Flash column chromatography was carried out using silica gel with particle size of $32-63 \mu \mathrm{m} .{ }^{1} \mathrm{H},{ }^{13} \mathrm{C}$ and ${ }^{31} \mathrm{P}$ NMR spectra were measured at 400, 100 and $162 \mathrm{MHz}$, respectively. HRMS was obtained using a TOF mass analyzer. RP HPLC: A C-18 analytical column (5 $\mu$ m diameter, $100 \AA$ pore size, $250 \times 3.2 \mathrm{~mm}$ dimension) was used. Solvent A: $0.1 \mathrm{M}$ triethylammonium acetate, 5\% acetonitrile. Solvent B: $90 \%$ acetonitrile. Profiles were generated by detection of absorbance of ODN at $260 \mathrm{~nm}$ using the following gradient system unless indicated otherwise: solvent B (0\%-45\%) in solvent A over 60 min followed by solvent B (45\%-100\%) in solvent A over $20 \mathrm{~min}$ at a flow rate of $0.5 \mathrm{~mL} / \mathrm{min}$. Ionexchange HPLC: An analytical Gen-Pak FAX $(100 \times 4.6 \mathrm{~mm})$ column was used. Solvent A: 25 mM Tris/Cl, $1 \mathrm{mM}$ EDTA, pH 8.0. Solvent B: $25 \mathrm{mM}$ Tris/Cl $1 \mathrm{mM}$ 
EDTA, 1.0 M NaCl, $\mathrm{pH}$ 8.0. The profile was generated by detection of absorbance of ODN at $260 \mathrm{~nm}$ using the gradient system: solvent B (10\%-75\%) in solvent A over 30 min followed by solvent $\mathrm{B}(75 \%)$ in solvent $\mathrm{A}$ for $30 \mathrm{~min}$ at a flow rate of 0.5 $\mathrm{mL} / \mathrm{min}$. Succinic ester linked DMTr-dT-lcaa-CPG (1000 Å pore size) and 5'-DMTr, 2-cyanoethyl phosphoramidites Pac-dA, benzoyl-dA, acetyl-dC, 4-isopropyl-Pac-dG, isobutyryl-dG, dT and other commonly used reagents for solid phase ODN synthesis were from commercial sources. The polymerization solution with a cross-linking ratio of 1:25 was used in all cases unless otherwise noted. The solution could be stored in a freezer at $-20{ }^{\circ} \mathrm{C}$ in dark for one month without affecting the results of catching by polymerization purification. High resolution MS and elemental analysis data for phosphoramidites were not collected due to their sensitivity to air and moisture.

\section{Methacrylamide 2.15}

The compound is known, [19] but a different procedure was used for its preparation. To a round-bottomed flask under a nitrogen atmosphere was added $\mathbf{2 . 1 4}$ (2.0 g, 19.0 mmol, 1.0 equiv), freshly distilled $\mathrm{CH}_{2} \mathrm{Cl}_{2}(60 \mathrm{~mL})$, and saturated $\mathrm{Na}_{2} \mathrm{CO}_{3}(60 \mathrm{~mL})$. The mixture was cooled to $0{ }^{\circ} \mathrm{C}$, and methacrolyl chloride (1.86 $\mathrm{mL}, 19.0 \mathrm{mmol}, 1.0$ equiv) was added via a syringe slowly with vigorous stirring. After addition, the reaction was allowed to proceed overnight while warming to $\mathrm{rt}$ gradually. The contents were then transferred into a separatory funnel. After removing the organic layer, the aqueous layer was extracted with $\mathrm{CH}_{2} \mathrm{Cl}_{2}(50 \mathrm{~mL} \times$

3). The combined organic phase was dried over anhydrous $\mathrm{Na}_{2} \mathrm{SO}_{4}$, and filtered. 
Volatiles were removed under reduced pressure. The crude product was purified with flash column chromatography $\left(\mathrm{SiO}_{2}, \mathrm{CH}_{2} \mathrm{Cl}_{2} / \mathrm{CH}_{3} \mathrm{OH}, 9: 1\right)$ giving 2.15 as a colorless oil (3.12 g, $18.0 \mathrm{mmol}, 95 \%): R_{f}=0.7\left(\mathrm{SiO}_{2}, \mathrm{CH}_{2} \mathrm{Cl}_{2} / \mathrm{CH}_{3} \mathrm{OH}, 9: 1\right)$.

\section{Phosphoramidite 2.11}

An oven-dried round-bottomed flask containing $2.15(0.5 \mathrm{~g}, 2.89 \mathrm{mmol}, 1.0$ equiv) and a magnetic stirring bar was evacuated and then refilled with nitrogen. The evacuation and nitrogen-filling cycle was repeated for three times. Freshly distilled $\mathrm{CH}_{2} \mathrm{Cl}_{2}(5 \mathrm{~mL})$ was added via a syringe forming a light yellow solution. 2Cyanoethyl- $N, N, N^{\prime}, N^{\prime}$-tetraisopropylphosphoramidite $(1.01 \mathrm{~mL}, 3.18 \mathrm{mmol}, 1.1$ equiv) and $1 H$-tetrazole ( $0.45 \mathrm{M}$ solution in $\mathrm{CH}_{3} \mathrm{CN}, 7.07 \mathrm{~mL}, 3.18 \mathrm{mmol}, 1.1$ equiv) were added slowly via syringes sequentially. After stirring at it for $2 \mathrm{~h}$, the reaction mixture was concentrated to dryness using a flow of nitrogen. The residue was purified with flash column chromatography $\left(\mathrm{SiO}_{2}\right.$, hexanes/ethyl acetate/Et $3 \mathrm{~N}$, 1:3:0.2) to give $\mathbf{2 . 1 1}$ as a colorless oil $(0.98 \mathrm{~g}, 2.62 \mathrm{mmol}, 90 \%): R_{f}=0.7\left(\mathrm{SiO}_{2}\right.$, hexanes/ethyl acetate/Et $3 \mathrm{~N}, 1: 3: 0.2) ;{ }^{1} \mathrm{H}$ NMR (400 MHz, $\left.\mathrm{CDCl}_{3}\right) \delta 6.29$ (br s, $1 \mathrm{H}$ ), $5.61(\mathrm{~s}, 1 \mathrm{H}), 5.24(\mathrm{~s}, 1 \mathrm{H}), 3.81-3.60(\mathrm{~m}, 4 \mathrm{H}), 3.57-3.50(\mathrm{~m}, 6 \mathrm{H}), 3.45-3.40(\mathrm{~m}, 2 \mathrm{H})$, $2.56(\mathrm{t}, J=6.4 \mathrm{~Hz}, 2 \mathrm{H}), 1.88(\mathrm{~s}, 3 \mathrm{H}), 1.18-1.04(\mathrm{~m}, 12 \mathrm{H}) ;{ }^{13} \mathrm{C}$ NMR $(100 \mathrm{MHz}$, $\left.\mathrm{CDCl}_{3}\right) \delta 168.6,140.2,119.6,117.8,71.2(\mathrm{~d}, J=7.6 \mathrm{~Hz}), 69.8,62.9(\mathrm{~d}, J=16.7 \mathrm{~Hz})$, $58.6(\mathrm{~d}, J=19.7 \mathrm{~Hz}), 43.2(\mathrm{~d}, J=12.1 \mathrm{~Hz}), 39.6,24.8,24.7 \times 2,24.66,20.55,20.47$, $18.8 ;{ }^{31} \mathrm{P}$ NMR $\left(162 \mathrm{MHz}, \mathrm{CDCl}_{3}\right) \delta 148.3$.

\section{Methacrylamide 2.17}

The compound is known, [22] but a different procedure, which was the same as that for the synthesis of compound 2.15, was used for its preparation. Reaction of 
compound 2.16, [20] (1.3 g, $8.71 \mathrm{mmol}, 1.0$ equiv) with methacrolyl chloride (851 $\mu \mathrm{L}, 8.71 \mathrm{mmol}, 1.0$ equiv) in saturated $\mathrm{Na}_{2} \mathrm{CO}_{3}(40 \mathrm{~mL})$ and $\mathrm{CH}_{2} \mathrm{Cl}_{2}(40 \mathrm{~mL})$ gave 2.17 as a colorless oil after flash column chromatography purification $\left(\mathrm{SiO}_{2}\right.$, $\left.\mathrm{CH}_{2} \mathrm{Cl}_{2} / \mathrm{CH}_{3} \mathrm{OH}, 9: 1 ; 1.6 \mathrm{~g}, 7.37 \mathrm{mmol}, 85 \%\right): R_{f}=0.6\left(\mathrm{SiO}_{2}, \mathrm{CH}_{2} \mathrm{Cl}_{2} / \mathrm{CH}_{3} \mathrm{OH}, 9: 1\right)$.

\section{Phosphoramidite 2.12}

The same procedure for the synthesis of phosphoramidite $\mathbf{2 . 1 1}$ was followed. Thus compound 2.17 (500 mg, $2.30 \mathrm{mmol}, 1.0$ equiv), 2-cyanoethyl- $N, N, N^{\prime}, N^{\prime}$ tetraisopropylphosphoramidite $(0.80 \mathrm{~mL}, 2.53 \mathrm{mmol}, 1.1$ equiv) and $1 H$-tetrazole (0.45 $\mathrm{M}$ in $\mathrm{CH}_{3} \mathrm{CN}, 5.60 \mathrm{~mL}, 2.53 \mathrm{mmol}, 1.1$ equiv) in $\mathrm{CH}_{2} \mathrm{Cl}_{2}(5 \mathrm{~mL})$ gave compound 2.12 as a colorless oil after flash column chromatography purification $\left(\mathrm{SiO}_{2}\right.$, hexanes/ethyl acetate/Et $\left.3 \mathrm{~N}, 1: 3: 0.2 ; 0.85 \mathrm{~g}, 2.04 \mathrm{mmol}, 88 \%\right): R_{f}=0.7\left(\mathrm{SiO}_{2}\right.$, hexanes/ethyl acetate/Et $\left.{ }_{3} \mathrm{~N}, 1: 3: 0.2\right) ;{ }^{1} \mathrm{H} \mathrm{NMR}\left(400 \mathrm{MHz}, \mathrm{CDCl}_{3}\right) \delta 6.33(\mathrm{br} \mathrm{s}, 1 \mathrm{H})$, $5.61(\mathrm{~s}, 1 \mathrm{H}), 5.22(\mathrm{~s}, 1 \mathrm{H}), 3.76-3.70(\mathrm{~m}, 4 \mathrm{H}), 3.58-3.49(\mathrm{~m}, 10 \mathrm{H}), 3.43-3.38(\mathrm{~m}, 2 \mathrm{H})$, $2.56(\mathrm{t}, J=6.4 \mathrm{~Hz}, 2 \mathrm{H}), 1.86(\mathrm{~s}, 3 \mathrm{H}), 1.10-1.07(\mathrm{~m}, 12 \mathrm{H}) ;{ }^{13} \mathrm{C}$ NMR $(100 \mathrm{MHz}$, $\left.\mathrm{CDCl}_{3}\right) \delta 168.5,140.2,119.6,117.9,71.4(\mathrm{~d}, J=7.6 \mathrm{~Hz}), 70.7,70.4,69.8,62.8(\mathrm{~d}, J$ $=18.2 \mathrm{~Hz}), 58.6(\mathrm{~d}, J=18.2 \mathrm{~Hz}), 43.2(\mathrm{~d}, J=12.1 \mathrm{~Hz}), 39.5,24.8,24.7 \times 2,24.66$, 20.5, 20.4, 18.8; ${ }^{31} \mathrm{P}$ NMR (162 MHz, $\left.\mathrm{CDCl}_{3}\right) \delta$ 149.5.

\section{Methacrylamide 2.19}

The same procedure for the synthesis of compound 2.15 was followed. Reaction of $\mathbf{2 . 1 8}$ [21] ( $2.0 \mathrm{~g}, 8.54 \mathrm{mmol}, 1.0$ equiv) with methacrolyl chloride ( 0.84 $\mathrm{mL}, 8.54 \mathrm{mmol}, 1.0$ equiv) in $\mathrm{CH}_{2} \mathrm{Cl}_{2}(80 \mathrm{~mL})$ and saturated $\mathrm{Na}_{2} \mathrm{CO}_{3}(60 \mathrm{~mL})$ gave 2.19 as a colorless oil (2.2 g, $7.28 \mathrm{mmol}, 85 \%)$ after flash column chromatography purification $\left(\mathrm{SiO}_{2}, \mathrm{CH}_{2} \mathrm{Cl}_{2} / \mathrm{CH}_{3} \mathrm{OH}, 9: 1\right): R_{f}=0.4\left(\mathrm{SiO}_{2}, \mathrm{CH}_{2} \mathrm{Cl}_{2} / \mathrm{CH}_{3} \mathrm{OH}, 9: 1\right) ;{ }^{1} \mathrm{H}$ 
NMR (400 MHz, $\left.\mathrm{CDCl}_{3}\right) \delta 6.94$ (br s, 1H), 6.80 (br s, 1H) 5.58 (s, 1H), 5.19 (s, 1H), 3.53-3.44 (m, 6H), 3.43-3.32 (m, 4H), 3.30-3.24 (m, 4H), $2.19(\mathrm{t}, J=7.0 \mathrm{~Hz}, 2 \mathrm{H})$, 1.81 (s, 3H), 1.74-1.66 (m, 2H); ${ }^{13} \mathrm{C}$ NMR (100 MHz, $\left.\mathrm{CDCl}_{3}\right) \delta$ 174.1, 169.1, 139.9, 120.1, 70.3, 69.92, 69.86, 61.7, 50.3, 39.6, 39.4, 33.4, 28.5, 18.7. HRMS (ESI, $[\mathrm{M}+\mathrm{H}]^{+}$) $m / z$ calcd for $\mathrm{C}_{14} \mathrm{H}_{27} \mathrm{~N}_{2} \mathrm{O}_{5} 303.1914$, found 303.1918 .

\section{Phosphoramidite 2.13}

The same procedure for the synthesis of phosphoramidite $\mathbf{2 . 1 1}$ was followed. Thus compound 2.19 (0.5 g $1.65 \mathrm{mmol}, 1.0$ equiv), 2-cyanoethyl- $N, N, N^{\prime}, N^{\prime}$ tetraisopropylphosphoramidite $(0.58 \mathrm{~mL}, 1.82 \mathrm{mmol}, 1.1$ equiv) and $1 H$-tetrazole (0.45 $\mathrm{M}$ in $\mathrm{CH}_{3} \mathrm{CN}, 4.05 \mathrm{~mL}, 1.82 \mathrm{mmol}, 1.1$ equiv) in $\mathrm{CH}_{2} \mathrm{Cl}_{2}(5 \mathrm{~mL})$ gave compound 2.13 as a colorless oil after flash column chromatography purification $\left(\mathrm{SiO}_{2}\right.$, hexanes/ethyl acetate/Et $\left.3 \mathrm{~N}, 1: 3: 0.2 ; 0.75 \mathrm{~g}, 1.49 \mathrm{mmol}, 90 \%\right): R_{f}=0.65\left(\mathrm{SiO}_{2}\right.$, hexanes/ethyl acetate/ $\left.\mathrm{Et}_{3} \mathrm{~N}, 1: 3: 0.2\right) ;{ }^{1} \mathrm{H} \mathrm{NMR}\left(400 \mathrm{MHz}, \mathrm{CDCl}_{3}\right) \delta 6.40(\mathrm{br} \mathrm{s}, 1 \mathrm{H})$, $6.22(\mathrm{br} \mathrm{s}, 1 \mathrm{H}), 5.64(\mathrm{~s}, 1 \mathrm{H}), 5.27(\mathrm{~s}, 1 \mathrm{H}), 3.84-3.69(\mathrm{~m}, 2 \mathrm{H}), 3.65-3.41(\mathrm{~m}, 14 \mathrm{H})$, 3.40-3.32 (m, 2H), $2.58(\mathrm{t}, J=6.0 \mathrm{~Hz}, 2 \mathrm{H}), 2.24(\mathrm{t}, J=7.4 \mathrm{~Hz}, 2 \mathrm{H}), 1.95-1.87(\mathrm{~m}$, 5H), 1.16-1.08 (m, 12H); ${ }^{13} \mathrm{C}$ NMR (100 MHz, $\left.\mathrm{CDCl}_{3}\right) \delta$ 172.8, 168.6, 140.2, 119.7, 118.0, 70.4, $69.9(\mathrm{~d}, J=18.3 \mathrm{~Hz}), 62.9(\mathrm{~d}, J=16.7 \mathrm{~Hz}), 58.4(\mathrm{~d}, J=19.8), 58.5,45.5$ (d, $J=6.0 \mathrm{~Hz}), 43.1(\mathrm{~d}, J=10.6), 39.5,39.3,33.1,27.3,24.8 \times 2,23.11,23.04,20.6$, 20.3, 18.8; ${ }^{31} \mathrm{P}$ NMR $\left(162 \mathrm{MHz}, \mathrm{CDCl}_{3}\right) \delta 149.5$.

Synthesis of ODN 2.20 Using Polymerizable Phosphoramidites 2.10-2.13 to Cap Failure Sequences - The General ODN Synthesis Procedure

The synthesis using $\mathbf{2 . 1 1}$ as the capping agent is described as an example. The phosphoramidite chemistry was used. CPG (1000 A pore size) with a LCAA-succinyl 55 
ester linkage was used as the solid support. The phosphoramidite monomers used were Pac-dA-CE, Ac-dC-CE, $i$-Pr-Pac-dG-CE and dT-CE. For capping failure sequences, a $0.15 \mathrm{M}$ solution of $\mathbf{2 . 1 1}$ in dry acetonitrile (distilled under nitrogen or from a commercial source) was placed on the $5^{\text {th }}$ phosphoramidite position. The two bottles normally used for storing $\mathrm{Ac}_{2} \mathrm{O}$ capping reagents were left empty. A new synthetic cycle was created by copying the standard $0.2 \mu \mathrm{mol}$ synthesis cycle into a new file. The steps for capping failure sequences with $\mathrm{Ac}_{2} \mathrm{O}$ were deleted. After the coupling steps, new steps for delivering $\mathbf{2 . 1 1}$ from the $5^{\text {th }}$ bottle position and $1 H$ tetrazole solution from the $9^{\text {th }}$ position (i.e. the same activator for the coupling steps) were added. These steps were the same as those in the coupling steps except that the base was from the $5^{\text {th }}$ bottle, the reagents were delivered for two additional times, and after each delivery a waiting of 90 seconds was added. More specifically, the solutions of 2.11 and $1 H$-tetrazole were delivered to column for 2.0 seconds, 1.5 seconds $\times 3$, and after each delivery, the reagents remained in the column for 90 seconds. After capping, steps for washing the column with acetonitrile and oxidation were added (Cycle 1 in supporting information). The synthesis was initiated using the above reagents and cycle on a $0.2 \mu \mathrm{mol}$ scale. In the last synthetic cycle, the 5'-DMTr group was removed. Cleavage and deprotection were carried out on the synthesizer with concentrated $\mathrm{NH}_{4} \mathrm{OH}(900 \mathrm{~min} \times 4)$ at $\mathrm{rt}$. The synthesized $\mathrm{ODN}$ is labeled as 2.10(c2.111). The same sequence was synthesized under the same conditions using polymerizable phosphoramidites 2.10 and $\mathbf{2 . 1 2 - 2 . 1 3}$ as capping reagent giving 2.20(c2.101), 2.20(c2.121) and 2.20(c2.13l), respectively. 


\section{Purification of ODN 2.20 by Polymerization of Failure Sequences - The General ODN Purification Procedure}

Purification of $\mathbf{2 . 2 0}(\mathbf{c 2 . 1 1 1})$ is described as an example. The ODN solution from the synthesizer was divided equally into four portions and dried in centrifuge tubes in a centrifugal vacuum concentrator. One portion was dissolved in $150 \mu \mathrm{L}$ water, $20 \mu \mathrm{L}$ was injected into RP HPLC to generate trace a (Figure 2.3). The remaining $130 \mu \mathrm{L}$ solution was transferred into a $25 \mathrm{~mL}$ 2-necked round-bottomed flask containing a magnetic stirring bar. The centrifuge tube was washed with water $(40 \mu \mathrm{L} \times 3)$, and the washes were transferred to the same flask. A polymerization solution $(250 \mu \mathrm{L}$, cross-linking ratio 1:25) was added via a pipette under positive nitrogen pressure. The solution was gently stirred under a nitrogen flow at rt. After 5 min, the nitrogen flow was stopped but stirring was continued under a nitrogen atmosphere. The polymerization reaction was then initiated with $\left(\mathrm{NH}_{4}\right)_{2} \mathrm{~S}_{2} \mathrm{O}_{8}(10 \%, 5$ $\mu \mathrm{L}$ ) and $N, N, N^{\prime}, N^{\prime}$-tetramethylethylenediamine (TMEDA, $5 \mu \mathrm{L}$ ), which were added sequentially via a pipette and a syringe, respectively. A clear gel was formed within 5 min and stirring stopped automatically. The gel was allowed to stand for $1 \mathrm{~h}$ to ensure complete polymerization.

The gel was broken into about 4 pieces with a clean spatula or preferably a knife with a sharp blade that does not grind the gel into fine pieces. To the flask, sufficient water that could cover all the gel $(\sim 3 \mathrm{~mL})$ was added. The mixture was stirred or shaken gently at $\mathrm{rt}$ for $3 \mathrm{~h}$. The supernatant was separated from the gel. The gel was further extracted with water $(3 \mathrm{~mL} \times 3)$ under the same conditions. After the volume was reduced in a centrifugal vacuum concentrator, the extracts were filtered into one 
$1.5 \mathrm{~mL}$ centrifuge tube that has a spin filtration function. The extracts were evaporated into dryness. To the residue, concentrated $\mathrm{NH}_{4} \mathrm{OH}(100 \mu \mathrm{L})$ was added. The tube was closed. After vortexing and centrifuging briefly, it was kept for 25 min on a heating block that was pre-heated to $80{ }^{\circ} \mathrm{C}$. The solution was then cooled to $\mathrm{rt}$, and $n \mathrm{BuOH}(900 \mu \mathrm{L})$ was added. The tube was vortexed for $30 \mathrm{sec}$ and centrifuged for $8 \mathrm{~min}$ at 14.1 relative centrifugal forces. The supernatant was removed carefully with a pipette. The residue was dried in a centrifugal vacuum concentrator shortly, and then dissolved in $130 \mu \mathrm{L}$ water. After vortexing and centrifuging shortly, $20 \mu \mathrm{L}$ was injected into RP HPLC to generate trace b (Figure 2.3). The recovery yield of the catching by polymerization process was estimated to be $93 \%$ by dividing the area of the peak at $19 \mathrm{~min}$ in trace b by that in trace a (Figure 2.3). Crude ODNs 2.20(c2.101), 2.20(c2.121) and 2.20(c2.131) were purified using the same procedure, and their recovery yields and purity are in Table 2.1. RP HPLC profiles are in supporting information.

Synthesis of ODN 2.20 Using Polymerizable Phosphoramidites 2.10-2.13 to Cap Failure Sequences with Shorter Capping Time

ODN 2.20 was synthesized again following the general ODN synthesis procedure described earlier on a $0.2 \mu \mathrm{mol}$ scale except that the capping time was decreased from 2.0 seconds, 1.5 seconds $\times 3$ deliveries and 90 seconds waiting after each delivery to 2.0 seconds, 1.5 seconds $\times 2$ deliveries and 30 seconds waiting after each delivery (Cycle 2 in supporting information). The synthesized ODNs are labeled as $2.20(\mathrm{c} 2.10 \mathrm{~s}), \mathbf{2 . 2 0}(\mathrm{c} 2.11 \mathrm{~s}), \mathbf{2 . 2 0}(\mathrm{c} 2.12 \mathrm{~s})$ and $2.20(\mathrm{c} 2.13 \mathrm{~s})$. 


\section{Purification of ODN 2.20 Synthesized Using Shorter Capping Time by Polymerization of Failure Sequences}

The general ODN purification procedure described earlier was used. The RP HPLC profiles are in supporting information. The recovery yields and purity are in Table 2.1.

\section{Diffusion Speed of ODN From Acrylamide Gel and Extraction Efficiency}

One fourth of the crude ODN 2.20(c2.11s) was subjected into the radical acrylamide polymerization conditions in a $25 \mathrm{~mL}$ 2-necked round-bottomed flask as described in the general ODN purification procedure. The polymerization solution used in the procedure had a 1:25 cross-linking ratio between the cross-linker $N, N^{\prime}$ methylenebis(acrylamide) and the polymerization monomer $N, N$-dimethylacrylamide. After complete polymerization, the gel was broken into several pieces with a clean spatula. Water $(3 \mathrm{~mL})$ was added to the gel, and the mixture was stirred gently with magnetic stirring bar. The aliquots of supernatant $(5 \mu \mathrm{L}$ each) were taken out via a pipette in every $10 \mathrm{~min}$ until $2 \mathrm{~h}$. The ODN concentrations in the aliquots were determined using a UV-Vis spectrophotometer at $260 \mathrm{~nm}$ (Table 2.2). At $2 \mathrm{~h}$, all the remaining supernatant were taken out and transferred into clean centrifuge tubes. The ODN concentration in the supernatant was determined with UV. To the gel was added water $(3 \mathrm{~mL})$ again and the mixture was gently stirred. After $2 \mathrm{~h}$, the supernatant was removed and the ODN concentration was determined with UV. The extraction and ODN quantification process was performed for three more times (Table 2.3). The ODN diffusion and extraction studies were repeated four more times using polymerization solutions with a different cross-linking ratio. One with a ratio of 1:50 
between $N, N^{\prime}$-methylenebis(acrylamide) and $N, N$-dimethylacrylamide, and the others 1:15, 1:7 and 1:2. The data were recorded in Tables 2.2 and 2.3.

\section{Synthesis of Long Sequence ODN 2.23}

The 61-mer ODN $\mathbf{2 . 2 3}$ was synthesized on a $0.2 \mu \mathrm{mol}$ scale using phosphoramidite $\mathbf{2 . 1 1}$ for capping failure sequences. The general synthesis procedure with shorter capping time (Cycle 2 in supporting information) was used except that a 30 seconds additional waiting step was added after the coupling steps to increase coupling yields.

\section{Purification of ODN 2.23 by Polymerization of Failure Sequences}

The general ODN purification procedure was used. The RP HPLC profiles from crude and purified ODN 2.23 were generated using a different buffer A that contained $10 \%$ urea under otherwise the same conditions described in the general methods section (supporting information). Recovery yield and purity are in Table 2.1. The purified 2.23 was analyzed with negative ESI MS using Waters' Tofspec-2E, calcd for $\mathrm{C}_{599} \mathrm{H}_{749} \mathrm{~N}_{235} \mathrm{O}_{361} \mathrm{P}_{60}$ 18875.1, found 18875.1. Image of the MS is included in supporting information.

\section{Synthesis of ODN 2.20 on a Larger Scale}

The general ODN synthesis procedure was followed except that a different synthesis cycle was used. To create the new cycle (Cycle 3 in supporting information), the standard $1 \mu \mathrm{mol}$ synthesis cycle provided by the manufacturer of the synthesizer was copied into a new file. The steps for capping failure sequences with $\mathrm{Ac}_{2} \mathrm{O}$ were deleted. After the coupling steps, new steps for delivering polymerizable phosphoramidite 2.11 ( $0.15 \mathrm{M}$ acetonitrile solution) from the $5^{\text {th }}$ phosphoramidite 60 
bottle position and $1 H$-tetrazole solution from the $9^{\text {th }}$ position (i.e. the same activator for the coupling steps) were added. These steps were the same as those in the coupling steps except that the base was from the $5^{\text {th }}$ bottle, one additional delivery of reagents was added, and after each delivery a waiting of 30 seconds was added. More specifically, the solutions of $\mathbf{2 . 1 1}$ and $1 H$-tetrazole were delivered to column for 2.5 seconds $\times 3$, and after each delivery, the reagents remained in the column for 30 seconds. Other modifications of the cycle were the same as detailed in the general synthesis procedure. The synthesis was performed using this new cycle at $1 \mu \mathrm{mol}$ scale following the conditions described in the general synthesis procedure. The ODN is labeled as $\mathbf{2 . 2 0}(\mathrm{c} 2.111 \mathrm{~s})$

\section{Purification of 2.20(c2.11ls) by Polymerization of Failure Sequences}

The general ODN purification procedure was followed. The $1 \mu \mathrm{mol}$ ODN was purified in one batch. The volume of the polymerization solution was 10 times of that used in $0.05 \mu \mathrm{mol}$ scale purification described in the general procedure. Extraction of full-length sequences from gel was achieved by water $(4 \mathrm{~mL} \times 4)$. HPLC profiles are in supporting information. Recovery yield and purity are in Table 2.1.

\section{Synthesis of ODN 2.20 with DCI as Activator}

ODN 2.20 was synthesized under the same conditions described for the synthesis of $\mathbf{2 . 2 0}(\mathrm{c} \mathbf{2 . 1 1} \mathrm{s})$ except for the following modifications. DCI (0.25 $\mathrm{M}$ in acetonitrile) instead of $1 H$-tetrazole was used as the activator for coupling and capping. The concentration of phosphoramidite monomers was lowered from $1.0 \mathrm{M}$ to $0.05 \mathrm{M}$. Capping was performed using a $0.1 \mathrm{M}$ acetonitrile solution of 2.11. The 
synthesis was performed in three $1 \mu$ mol columns simultaneously. The ODN is labeled as $\mathbf{2 . 2 0}$ (c2.11dci).

\section{Purification of 2.20(c2.11dci) by Polymerization of Failure Sequences}

The $3 \mu \mathrm{mol}$ ODN was purified in one batch following the procedure for the purification of $\mathbf{2 . 2 0}(\mathrm{c} 2.111 \mathrm{~s})$. The volumes of the polymerization solution and extraction solutions were increased proportionally (i.e. 3 times of those for the $1 \mu \mathrm{mol}$ 2.20(c2.11ls) purification). The volumes of the polymerization initiators and the reagents for ODN precipitation from $\mathrm{NH}_{4} \mathrm{OH}$ solution by $n \mathrm{BuOH}$ were not changed. The RP HPLC profiles from crude and purified ODN 2.20(c2.11dci) including one that was generated by UV detection at $210 \mathrm{~nm}$ from the purified ODN are included in supporting information. Recovery yields and purity are in Table 2.1.

\section{Synthesis of Mixture ODN 2.25}

The general ODN synthesis procedure with a shorter capping time was followed (Cycle 2 in supporting information). The synthesis was carried out on a 0.2 $\mu$ mol scale using polymerizable phosphoramidite $\mathbf{2 . 1 1}$ for capping failure sequences. To synthesize the mixture ODN, the solution of Pac-dA-CE, Ac-dC-CE, $i$-Pr-Pac-dG$\mathrm{CE}$ and $\mathrm{dT}-\mathrm{CE}$ in 1:1:1:1 mole ratio was used as the phosphoramidite monomers in the coupling steps in all synthesis cycles. The cycle was executed 20 times to give the 20-mer ODN mixture 2.25. At the end of synthesis, the DMTr group was removed. The ODN were cleaved and deprotected with concentrated $\mathrm{NH}_{4} \mathrm{OH}$ at $\mathrm{rt}$ on synthesizer. 


\section{Purification of ODN Mixture 2.25 by Polymerization of Failure Sequences}

The general ODN purification procedure was used to remove failure sequences. The RP HPLC profiles from crude and purified ODN 2.25 (only failure sequences were removed; theoretically it still contained $4{ }^{19}$ sequences) are included in supporting information. The recovery yield of the purification process was estimated to be $78 \%$.

\section{ODN Stability Studies Through Nuclease Digestion Followed by RP HPLC Analysis}

To a $1.5 \mathrm{~mL}$ centrifuge tube, which contained $18 \mu \mathrm{g}$ ODN 2.20 purified by catching failure sequences by polymerization, was added $29 \mu \mathrm{L}$ master mixture solution containing 16.45 unit SVP (Worthington Biochemical Corporation), 0.4 unit BAP (TaKaRa Bio Inc.), and $1 \times$ of BAP buffer. The tube was vortexed and centrifuged briefly to mix thoroughly and to bring the master mixture to the bottom of the tube. The mixture was then incubated at $37^{\circ} \mathrm{C}$ for $24 \mathrm{~h}$. The digested ODN was then prepared for RP HPLC analysis following reported procedure.[27] Briefly, sodium acetate buffer $(3 \mathrm{M}, 4 \mu \mathrm{L})$ and ethanol $(100 \mu \mathrm{L})$ were added into the tube. The tube was then vortexed shortly and chilled on dry ice for 10 min. After centrifuging for $5 \mathrm{~min}$ at 14.1 relative centrifugal force, the supernatant was carefully transferred into another $1.5 \mathrm{~mL}$ centrifuge tube. To the tube was added ethanol (300 $\mu \mathrm{L}$ ), and the contents were vortexed and chilled on dry ice again for $10 \mathrm{~min}$. The tube was centrifuged for $5 \mathrm{~min}$, and the supernatant was transferred into a new $1.5 \mathrm{~mL}$ centrifuge tube. After drying in a centrifugal vacuum concentrator, the nucleosides were dissolved in water $(30 \mu \mathrm{L})$. The sample $(20 \mu \mathrm{L})$ was analyzed with RP HPLC 
under the conditions described in the General section except that the gradient system was changed to: solvent A (100\%) for 5 min, solvent B (0\%-10\%) in solvent A over 30 min, followed by solvent B (10\%-100\%) in solvent A over 25 min. The resulting profile is shown in Figure. 2.4 (trace a). The same ODN digestion and RP HPLC analysis procedure was applied to a control ODN $(18 \mu \mathrm{g})$ from a commercial source purified with preparative RP HPLC (trace b), the mixture ODN 2.25 (33 $\mu$ g, trace d), and a blank control (supporting information). The control ODN $2.24(9 \mu \mathrm{g})$ was synthesized and digested following known procedure (trace c).[28]

\section{Effect of One-Month Old Capping Agent on Purification}

For the catching by polymerization purification technology to be practically useful, it is important that capping phosphoramidites stored for a reasonable time have a similar capping efficiency as freshly prepared ones. For this reason, we stored compound 2.11 in a freezer under a nitrogen atmosphere at $-20^{\circ} \mathrm{C}$. After one month, its acetonitrile solution was prepared and used for the synthesis ODN 2.20 for capping failure sequences. The synthesis procedure with shortened capping time (Cycle 2) was used. The crude ODN, which is labeled as 2.20(c2.11o), was purified with the catching failure sequences by polymerization method. RP HPLC analysis (see this supporting information) indicated that the purification results were the same as with freshly prepared capping agents. The recovery yield of the purification process was estimated to be $87 \%$. The purity is $92 \%$.

\section{Effect of Radical Scavenger on Purification}

For one time, when pure phosphoramidite $\mathbf{2 . 1 0}$, which was a thick oil, was dried under high vacuum at room temperature overnight, it became a gel. The reason 
is that under such conditions, the amount of oxygen, which serves as a radical scavenger, was significantly reduced, and radical polymerization occurred. To avoid premature radical polymerization, since then all capping phosphoramidites (2.102.13) were dried over drierite in a dessicator under a relatively low vacuum (achieved using an oil pump for an appropriate time and then close the dessicator) at $-20^{\circ} \mathrm{C}$ in a freezer overnight. With the new drying method, we never met premature polymerization problem again. In the case of the solution of the methacrylamide phosphoramidites (2.10-2.13) in acetonitrile, premature polymerization never occurred in our hands. They are stable for at least three days on a synthesizer or in a freezer $\left(-20^{\circ} \mathrm{C}\right)$ under nitrogen. Despite these favorable observations, potential users of the technology may still be interested to know if the capping steps during ODN synthesis are compatible with radical polymerization inhibitors in the capping solution. To clarify, ODN $\mathbf{2 . 2 0}$ was synthesized using the procedure with shortened capping time (Cycle 2). The solution of $2.11(0.15 \mathrm{M})$ in acetonitrile with $500 \mathrm{ppm}$ 2,6-di-tert-4-methylphenol was used for capping. The crude ODN was purified by catching failure sequences in the usual way. RP HPLC analysis (see this supporting information) revealed that the ODN, which is labeled as $\mathbf{2 . 2 0}$ (c2.11i), was pure indicating that the radical scavenger studied did not adversely affect the purification results. The recovery yield of the purification process was estimated to be $97 \%$. The purity is $98 \%$. 


\section{Effect of Harsher Deprotecting Conditions on Purification}

In our studies so far, the exo-amino groups on adenine and guanine were protected with phenoxyacetyl (Pac) and isopropyl-phenoxyacetyl ( $i$-Pr-Pac) groups, respectively. These groups along with the acetyl group on cytosine were removed with concentrated $\mathrm{NH}_{4} \mathrm{OH}$ at room temperature after synthesis. This protecting strategy has the advantage of avoiding deprotection under ammonia pressure in a sealed vial at elevated temperature. In the less expensive and more commonly used ODN synthesis methods, adenine and guanine are protected with benzoyl and isobutyryl groups, respectively, and deprotection are usually achieved with concentrated $\mathrm{NH}_{4} \mathrm{OH}$ at elevated temperature. Previously, we showed that the harsher deprotecting conditions were compatible with the catching full-length sequences by polymerization ODN purification technology (Y. Yuan et al, RSC Adv., 2012, 2, 2803). Here, we show that the conditions are also compatible with the catching failure sequences method. The ODN $\mathbf{2 . 2 0}$ was synthesized on a $0.2 \mu \mathrm{mol}$ scale using the more commonly used protecting strategy (benzoyl for $\mathrm{dA}$, isobutyryl for $\mathrm{dG}$, and Ac for $\mathrm{dC}$ ). The synthetic cycle with shortened capping time described earlier (Cycle 2) was used for the synthesis, and phosphoramidite 2.11 was employed for capping failure sequences. At the end of synthesis, DMTr group was removed, and cleavage was performed on the synthesizer with concentrated $\mathrm{NH}_{4} \mathrm{OH}(900 \mathrm{~min} \times 4)$ at $\mathrm{rt}$. Deprotection was achieved by heating the concentrated $\mathrm{NH}_{4} \mathrm{OH}$ solution in a screw capped vial at $65^{\circ} \mathrm{C}$ for $8 \mathrm{~h}$. The ODN, which was labeled as 2.20(c2.11c), was purified by catching failure sequences by polymerization as usual. RP HPLC analysis showed that the ODN was pure (see this supporting information). The recovery yield 
of the purification process was estimated to be $76 \%$. The purity is $93 \%$. The results indicate that the catching failure sequences by polymerization purification method is equally effective when protecting groups that require harsher deprotecting conditions are used during ODN synthesis.

\section{Capping with Lower Concentration Polymerizable Phosphoramidite Solution}

In our preliminary communication, we used a capping solution with a concentration of $0.2 \mathrm{M}$ to cap failure sequences (S. Fang et al, Chem. Commun., 2011, 47, 1345). In the present work, we reduced the concentration of capping solution to $0.15 \mathrm{M}$. Good purification results were obtained in both cases. In the coupling steps, the concentration of nucleoside phosphoramidite monomers we used was $0.1 \mathrm{M}$. We were interested in further reducing the concentration of the capping solution to this value. For the purpose, ODN 2.20 was synthesized using the procedure with shortened capping time (Cycle 2). Capping failure sequences was performed with a $0.1 \mathrm{M}$ solution of $\mathbf{2 . 1 0}$. The resulting ODN, which is labeled as 2.20(c2.10lc), was purified by catching failure sequences by polymerization as usual. RP HPLC analysis showed that the ODN was equally pure (see this supporting information). The recovery yield of ODN was estimated to be $90 \%$. The purity is $99 \%$.

\section{Purification by Polymerization in Air}

So far in all of our studies, the polymerization step, in which failure sequences were incorporated into a polyacrylamide gel, was performed under a nitrogen atmosphere to minimize the chances of termination of polymerization by oxygen. This may not be convenient for some applications such as high throughput 
purification. Noting that excess initiators were used and cross-linking could connect terminated polymer segments, we tested to perform the step in air in a $1.5 \mathrm{~mL}$ centrifuge tube. A portion of ODN 2.20(c2.11s) in a centrifuge tube was simply dissolved in water and polymerized by adding polymerization solution and initiators to the tube directly. Interestingly, the polymerization speed was not found slower than that under nitrogen atmosphere, and the gel was formed within 5 min. After waiting for complete polymerization for $1 \mathrm{~h}$, extraction of full-length sequences with water was also carried out directly in the same tube. The remaining steps were the same as in the general purification procedure. RP HPLC showed that the ODN was equally pure (see this supporting information). The recovery yield was estimated to be $87 \%$. The purity is $100 \%$. Polymerization under nitrogen in large scale purifications should be quite convenient, and is not expected to add any significant cost to the process. However, for high throughput purification, handling many samples at the same time under a nitrogen atmosphere may require special equipment. Therefore, the results are important for applying the technology in high throughput ODN purification.

\subsection{Conclusion}

Several details of the catching failure sequences by polymerization ODN purification technology were investigated. We found that the four methacrylamide phosphoramidites 2.10-2.13 were similarly effective for capping failure sequences in the purification technique. However, because 2.10 and 2.11 are easier to prepare, they are the best choices. The studies on the diffusion speeds of ODN from polyacrylamide gel to solution indicate that extraction of ODN from gel is efficient. 
Normally, three extractions will recover more than $90 \%$ ODN. Contrary to our prediction, ODN extraction efficiency is higher in the case of gels with higher crosslinking. This is a favorable finding for the technology because gels with higher crosslinking are harder and easier to work with. The technology was successfully applied for the purification of a long sequence and purification at a larger scale. Capping failure sequences with older methacrylamide phosphoramidite and phosphoramidite solution containing a radical scavenger were also studied and were found that they have little adverse effects on the purification results. The more commonly used exoamino nucleobases protecting groups that require harsher conditions to remove were tested in the ODN purification method. They were found equally compatible with the technology as the more labile phenoxyacetyl protecting groups. Polymerization in air is more convenient than under a nitrogen atmosphere. This is especially important for high throughput purification. We demonstrated that it does not affect purification results. Finally and most importantly, we provided further evidences to support that ODNs are stable under free radical acrylamide polymerization conditions. This study is most important in the context of ODN drug purification. 


\section{Acknowledgements}

Financial support from US NSF (CHE-0647129 and CHE-1111192), Michigan Universities Commercialization Initiative, MTU Research Excellence Fund (REF-TC), MTU Chemistry Department, MTU Biotech Research Center, and The Royal Thai Government Scholarship (S. F.); the assistance from Mr. Dean W. Seppala (electronics) and Mr. Jerry L. Lutz (NMR); and NSF equipment grants (CHE-9512445 and CH-1048655) are all gratefully acknowledged. 


\section{References and Notes}

1. Hughes, R.A., A.E. Miklos, and A.D. Ellington, Gene synthesis: methods and applications. Methods Enzymol, 2011, 498: p. 277-309.

2. Polymerization in the tube over the filter was found less efficient. This problem can be solved by rinsing the upper tube in a solvent such as ethanol overnight or washing three times.

3. Yu, T., X. Bao, W. Piao, et al., Recent patents on oligonucleotide synthesis and gene synthesis. Recent patents on DNA \& gene sequences, 2012, 6(1): p. $10-21$.

4. Schulte, M., N. Luhring, A. Keil, et al., Purification of DMT-on oligonucleotide by simulated moving-bed (SMB) chromatography. Organic Process Research \& Development, 2005, 9(2): p. 212-215.

5. Agbavwe, C., C. Kim, D. Hong, et al., Efficiency, error and yield in lightdirected maskless synthesis of DNA microarrays. Journal of nanobiotechnology, 2011, 9(1): p. 1-17.

6. Tian, J., K. Ma, and I. Saaem, Advancing high-throughput gene synthesis technology. Molecular Biosystems, 2009, 5(7): p. 714-722.

7. Aitken, S. and E. Anderson, Oligoprep PVA support for oligonucleotide synthesis in columns on a scale up to $10 \mathrm{mu}$ mol. Nucleosides Nucleotides \& Nucleic Acids, 2007, 26(8-9): p. 931-934.

8. Pearson, W.H., D.A. Berry, P. Stoy, et al., Fluorous affinity purification of oligonucleotides. Journal of Organic Chemistry, 2005, 70(18): p. 7114-7122. 
9. Sproat, B.S., T. Rupp, N. Menhardt, et al., Fast and simple purification of chemically modified hammerhead ribozymes using a lipophilic capture tag. Nucleic Acids Research, 1999, 27(8): p. 1950-1955.

10. Beller, C. and W. Bannwarth, Noncovalent attachment of nucleotides by fluorous fluorous interactions: Application to a simple purification principle for synthetic DNA fragments. Helvetica Chimica Acta, 2005, 88(1): p. 171179.

11. Fang, S.Y. and D.E. Bergstrom, Reversible biotinylation phosphoramidite for 5 '-end-labeling, phosphorylation, and affinity purification of synthetic Oligonucleotides. Bioconjugate Chemistry, 2003, 14(1): p. 80-85.

12. Fang, S.Y. and D.E. Bergstrom, Fluoride-cleavable biotinylation phosphoramidite for 5 '-end-labeling and affinity purification of synthetic oligonucleotides. Nucleic Acids Research, 2003, 31(2): p. 708-715.

13. Fang, S.Y. and D.E. Bergstrom, Reversible 5 '-end biotinylation and affinity purification of synthetic RNA. Tetrahedron Letters, 2004, 45(43): p. 79877990.

14. Pieken, W., A. Wolter, and M. Leuck, Methods for the integrated synthesis and purification of oligonucleotides. 2003, Google Patents.

15. Fang, S. and S. Fueangfung, Scalable Synthetic Oligodeoxynucleotide Purification with Use of a Catching by Polymerization, Washing, and Releasing Approach. Organic Letters, 2010, 12(16): p. 3720-3723. 
16. Fang, S., S. Fueangfung, X. Lin, et al., Synthetic oligodeoxynucleotide purification by polymerization of failure sequences. Chemical Communications, 2011, 47(4): p. 1345-1347.

17. Yuan, Y., S. Fueangfung, X. Lin, et al., Synthetic 5 '-phosphorylated oligodeoxynucleotide purification through catching full-length sequences by polymerization. Rsc Advances, 2012, 2(7): p. 2803-2808.

18. Sawadogo, M. and M.W. Vandyke, A rapid method for the purification of deprotected olligodexynucleotides. Nucleic Acids Research, 1991, 19(3): p. 674-674.

19. Gomez-Valdemoro, A., M. Trigo, S. Ibeas, et al., Acrylic Copolymers with Pendant 1,2,4-Triazole Moieties as Colorimetric Sensory Materials and Solid Phases for the Removal and Sensing of Cations from Aqueous Media. Journal of Polymer Science Part a-Polymer Chemistry, 2011, 49(17): p. 3817-3825.

20. Liu, L.-H., H. Dietsch, P. Schurtenberger, et al., Photoinitiated Coupling of Unmodified Monosaccharides to Iron Oxide Nanoparticles for Sensing Proteins and Bacteria. Bioconjugate Chemistry, 2009, 20(7): p. 1349-1355.

21. Tang, W. and S. Fang, Mono-acylation of symmetric diamines in the presence of water. Tetrahedron Letters, 2008, 49(41): p. 6003-6006.

22. Dalven, P.I., J.R. Hildebrandt, A. Shamir, et al., Acrylonitrile-based copolymers-synthesis, characterization, and formation of ultrafiltration membranes utilized for the immobilization of proteins. Journal of Applied Polymer Science, 1985, 30(3): p. 1113-1132. 
23. Fang, S., S. Fueangfung, and Y. Yuan, Purification of Synthetic Oligonucleotides via Catching by Polymerization, in Current Protocols in Nucleic Acid Chemistry. 2012, John Wiley \& Sons, Inc.

24. Baeissa, A., N. Dave, B.D. Smith, et al., DNA-Functionalized Monolithic Hydrogels and Gold Nanoparticles for Colorimetric DNA Detection. Acs Applied Materials \& Interfaces, 2010, 2(12): p. 3594-3600.

25. Helwa, Y., N. Dave, R. Froidevaux, et al., Aptamer-Functionalized Hydrogel Microparticles for Fast Visual Detection of Mercury(II) and Adenosine. Acs Applied Materials \& Interfaces, 2012, 4(4): p. 2228-2233.

26. Toriello, N.M., C.N. Liu, R.G. Blazej, et al., Integrated affinity capture, purification, and capillary electrophoresis microdevice for quantitative double-stranded DNA analysis. Analytical Chemistry, 2007, 79(22): p. 85498556.

27. Andrus, A. and R.G. Kuimelis, Base Composition Analysis of Nucleosides Using HPLC, in Current Protocols in Nucleic Acid Chemistry. 2001, John Wiley \& Sons, Inc.

28. Bodepudi, V., S. Shibutani, and F. Johnson, Syntheis of 2'-deoxy-7,8-dihydro8-oxoguanosine and 2'deoxy-7,8-dihydro-8-oxoadenosine and their incorporation into oligomeric DNA. Chemical Research in Toxicology, 1992, 5(5): p. 608-617. 


\title{
Chapter 3
}

\section{A Highly Convenient Procedure for Oligodeoxynucleotide Purification ${ }^{1}$}

\author{
Durga Pokharel, Shiyue Fang \\ Department of Chemistry, Michigan Technological University, 1400 Townsend Drive, \\ Houghton, MI 49931 USA \\ Tel.: +1 906487 2023; fax: +1 9064872061 . \\ Email: shifang@mtu.edu
}

\footnotetext{
${ }^{1}$ This material contained in this chapter was previously published in The Open Organic Chemistry Journal, 2014, 8, 15-18. DOI: 10.2174/1874095201408010015 Reproduced by permission from Bentham Open Journal. http://benthamopen.com/toocj/openaccess2.htm
} 
The idea of the project in chapter 3 was conceived by Dr. Shiyue Fang. All experiments in "A Highly Convenient Procedure for Oligodeoxynucleotide Purification" were carried out by Durga P. Pokharel. Dr. Fang revised writing of Durga P. Pokharel

Apporved by advisor Dr. Shiyue Fang 


\begin{abstract}
Purification of synthetic oligodeoxynucleotides (ODNs) is simply achieved by capping failure sequences with a polymerizable phosphoramidite followed by polymerization. In this article, the reduction of the amount of polymerization monomer to drastically increase ODN extraction efficiency, the use of a centrifugal filter unit to ease the extraction process and the notification of using fresh phosphoramidite solutions are described. In addition, further evidence to support the purity of ODN and discussions of ODN stability under radical polymerization conditions are provided.
\end{abstract}




\subsection{Introduction}

Synthetic oligodeoxynucleotides (ODNs) have found wide applications in areas such as molecular biology, synthetic biology and antisense drug development.[1] Their syntheses have been automated. Large scale and high throughput syntheses are both possible.[2-6] During synthesis, the first nucleoside is attached to a solid support. Subsequent nucleosides are added sequentially to give the full-length ODN. The coupling reactions are highly efficient, but cannot be $100 \%$, which inevitably generates failure sequences. These failure sequences are usually capped with acetic anhydride to ease purification. At the end of synthesis, the ODN is cleaved from support and fully deprotected. The product contains failure sequences and small molecules besides full-length sequences, and has to be purified for most applications and ideally for all applications. The small molecules are easy to remove because they are neutral and ODNs are anionic. Removal of failure sequences is, however, more difficult. Currently, the most widely used method is HPLC. Drawbacks include high cost of instrument and column, labor intensiveness, and high waste to product ratio. The method is difficult to automate, expensive to scale up, and unsuitable for high throughput purification. Other methods have been developed to solve the problems[7-13], but still have various shortcomings.[14]

We recently reported a catching failure sequences by polymerization method for ODN purification.[14, 15] In this method, the failure sequences are capped with a polymerizable phosphoramidite such as $\mathbf{3 . 1}$ (Figure 3.1). Purification is achieved by copolymerization with $N, N$-dimethylacrylamide. The failure sequences are 
incorporated into polymer. The full-length sequences and small molecules remain in solution and polymer matrix. Extraction with water and $n \mathrm{BuOH}$ precipitation gives pure ODN. In this letter, we report several highly important refinements of the technology. In our initial procedure, large excess of polymerization monomer was used. One refinement is to reduce this amount. This refinement drastically increased the extraction efficiency of ODN from polymer. Previously, extraction of ODN was accomplished by pipetting supernatant, which was inconvenient. The second refinement is to carry out polymerization in a centrifugal filter unit and to perform extraction by simple spins. Besides these refinements, further evidences to prove the purity of ODN, and the explanation of the stability of ODN under radical polymerization conditions are provided.

The greatly simplified procedure is demonstrated by the purification of the 20mer ODN 5'-TCA TTG CTG CTT AGA CCG CT-3' (3.2). The ODN synthesis procedure, which includes capping failure sequences by 3.1, was similar as previously described.[15] Briefly, the synthesizer manufacturer (ABI 394) suggested $1 \mu \mathrm{mol}$ DNA synthesis cycle was copied to create a new cycle. The steps for capping with acetic anhydride were replaced with those for capping with $\mathbf{1}$, which were achieved by delivering 3.1 and activator to column. The delivery method was the same as the coupling steps except that an additional delivery was added $(2.5$ seconds $\times 3)$ and after each delivery a wait of 30 seconds was added. Following capping, three washes (acetonitrile to column, 10 seconds; reverse flush, 6 seconds) were added. The synthesis was set up as usual with 3.1 (0.1 $\mathrm{M}$ in acetonitrile)[15] being placed in bottle 5. 4,5-Dicyanoimidazole $(0.25 \mathrm{M}$ in acetonitrile $)$ was used as activator. 79 
Because the catching by polymerization method can isolate full-length ODN from very complex mixture, we lowered the concentration of phosphoramidite monomer (Pac-dA, acetyl-dC, 4-isopropyl-Pac-dG, dT) solutions from the usually used 0.1 M to $0.05 \mathrm{M}$ (in acetonitrile). At the end of synthesis, detritylation was performed. Cleavage and deprotection were carried out with concentrated $\mathrm{NH}_{4} \mathrm{OH}$ at room temperature on synthesizer $(15 \min \times 4$, then $2 \mathrm{~h}$ ). The solution was divided into four portions and dried in 1.5 centrifugal tubes. One portion was dissolved in $600 \mu \mathrm{L}$ water, $20 \mu \mathrm{L}$ was injected in to HPLC to give the crude profile (trace A, Figure 3.2).

Another portion of the crude $3.2(\sim 0.25 \mu \mathrm{mol})$ was dissolved in $50 \mu \mathrm{L}$ water. Short vortex and spin were performed to ensure complete dissolution and bring down solution to bottom. To the solution was added $6 \mu \mathrm{L}$ polymerization solution $(0.37 \mathrm{M}$ $N, N^{\prime}$-methylenebisacrylamide and $3.7 \mathrm{M} N, N$-dimethylacrylamide) followed by a short vortex and spin. The molar ratio of the polymerization monomer over ODN is $\sim 100 / 1$. The initiators_ $\left(\mathrm{NH}_{4}\right)_{2} \mathrm{~S}_{2} \mathrm{O}_{8}(5 \%, 5 \mu \mathrm{L})$ and TMEDA $(0.66 \mathrm{M}, 5 \mu \mathrm{L})$ were then added. After a short vortex and spin, the mixture was transferred to the center of the filter in a centrifugal filter unit ( $2 \mathrm{~mL}$, pore size $7-20 \mu \mathrm{m}$, Aldrich) immediately in one portion (Figure 3.1).[16] 


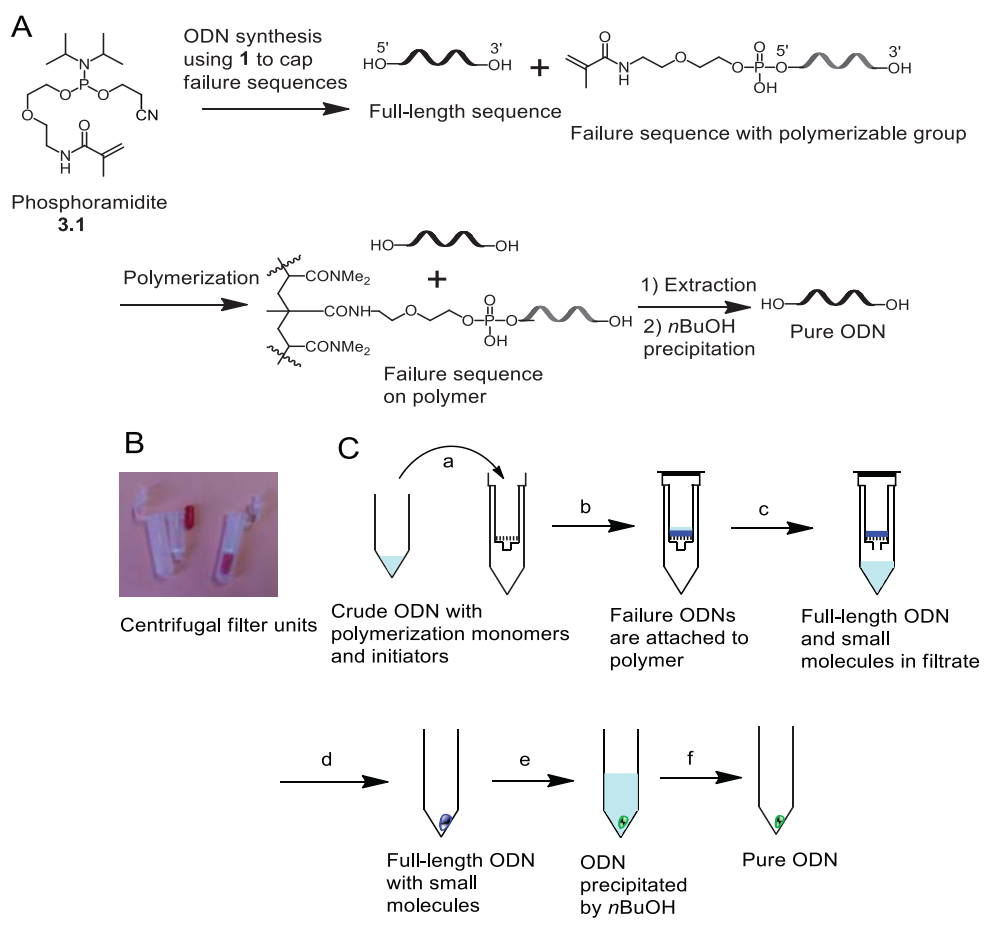

Figure 3.1. The catching failure sequences by polymerization ODN purification technology. (A) Working principle. (B) Picture of centrifugal filter units. (C) Workflow: (a) To crude ODN in a centrifugal tube are added polymerization monomer, cross-linker and initiators. After mixing, the contents are transferred to the upper tube of a centrifugal filter unit. (b) The cap is closed and the mixture is allowed to polymerize for $1 \mathrm{~h}$. (c) The gel is cut into several pieces and the supernatant is collected in the lower tube by spin. To the gel was added water. After standing at room temperature for $10 \mathrm{~min}$, spin again. The extraction is repeated two more times. (d) The upper tube is removed, and the filtrate is evaporated to dryness. (e) To the residue is added concentrated $\mathrm{NH}_{4} \mathrm{OH}$, warmed for 15 min at $80{ }^{\circ} \mathrm{C}$ in a heating block. After cooling to room temperature, $n \mathrm{BuOH}$ is added. The mixture is vortexed and then spun for 2 min. (f) The supernatant is removed by a pipette. 


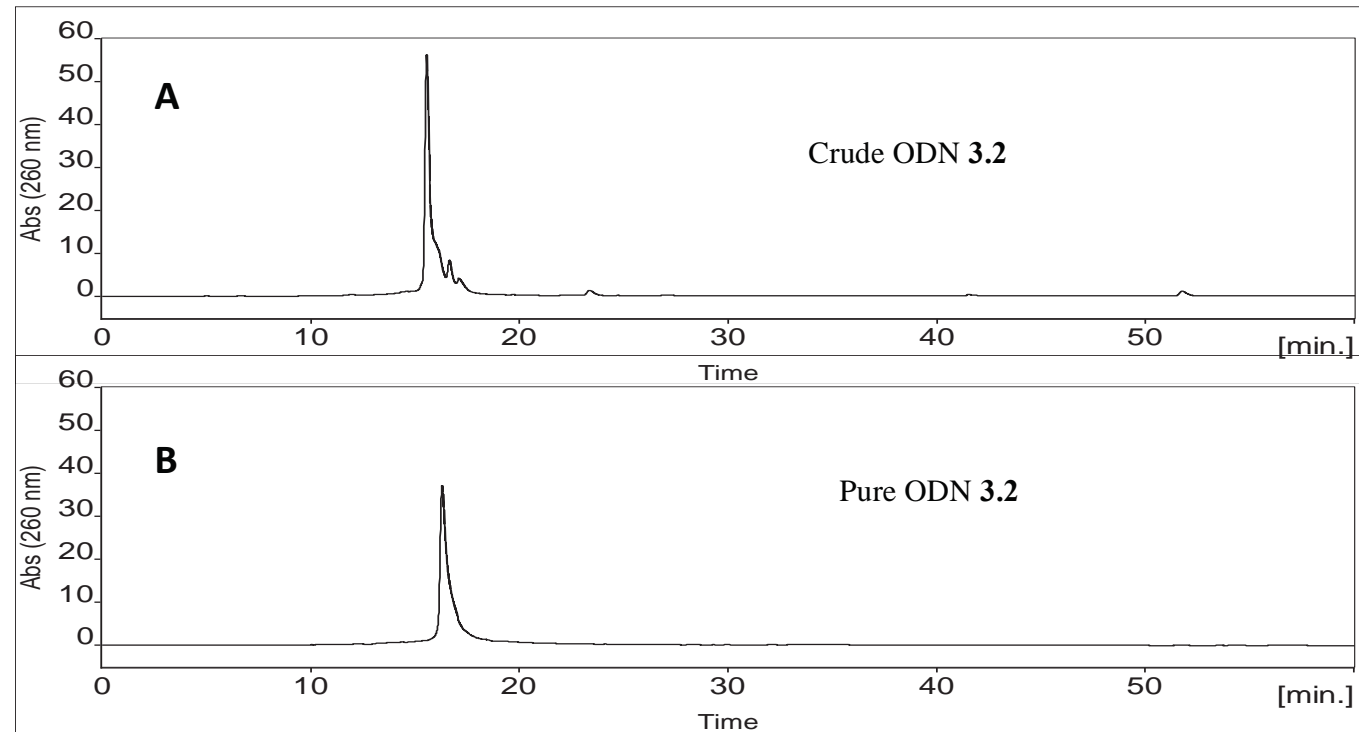

Figure 3.2. HPLC profiles of ODN 3.2. Conditions: column, C-18, $5 \mu \mathrm{m}, 100 \AA$, 250 $\times 3.20 \mathrm{~mm}$; solvent A, 0.1 M triethylammonium acetate, $5 \%$ acetonitrile; solvent $\mathrm{B}$, 90\% acetonitrile; gradient, solvent B (0\%-45\%) in solvent A over 60 min; flow rate, $0.5 \mathrm{~mL} / \mathrm{min}$; detection, $260 \mathrm{~nm}$.

It is suggested to close the bottom of the tube, but we found that the solution would not leak even without closing. Care needs to be taken to transfer all contents and to avoid splashing, which is easily achievable by manipulating the pipette with a steady force as opposed to abrupt sucking and pushing. The unit was closed and the mixture was allowed to polymerize for 1 hour. The gel was cut into about four pieces, and the bottom of the upper tube was opened. The unit was spun for about 30 seconds, which separated the supernatant from gel. To the upper tube was added 50 $\mu \mathrm{L}$ water, which should cover all the gel. After standing for 10 minutes, the unit was spun. The extraction was repeated two more times. The supernatants were evaporated to dryness. To the residue, $100 \mu \mathrm{L}$ concentrated $\mathrm{NH}_{4} \mathrm{OH}$ was added, and the mixture 
was heated to $80{ }^{\circ} \mathrm{C}$ for 15 minutes on a heating block (CAUTION: safety goggles and face shield are needed for potential explosion). After cooling to room temperature, $900 \mu \mathrm{L} n \mathrm{BuOH}$ was added. The mixture was vortexed shortly and spun for 2 minutes. The supernatant was removed with a pipette. The white flake was pure ODN. To determine purity and recovery yield, it was dissolved in $600 \mu \mathrm{L}$ water, and $20 \mu \mathrm{L}$ was injected into HPLC to give trace B (Figure 3.2). The ODN was $100 \%$ pure. The recovery yield was estimated to be $97 \%$ by dividing the area of the peak at 15.5 minutes in trace B by that in trace A. To see if more ODN could be obtained from the gel, the extraction and precipitation process was repeated. HPLC analysis did not show any ODN (supporting information), which indicated that three extractions in a time as short as 30 minutes were sufficient. We also performed a blank control experiment in which no ODN was used. We did not see any residue. HPLC analysis did not found any UV active material.

To confirm that the ODN peak in trace B does not contain any failure sequences, we synthesized the 19-mer ODN 5'-CAT TGC TGC TTA GAC CGC T-3' (3.3), which was identical to $\mathbf{3 . 2}$ except that the $20^{\text {th }}$ base was not added. The ODN was purified by catching by polymerization. Co-injection of $\mathbf{3 . 2}$ and $\mathbf{3 . 3}$ gave a broadened peak. When a slower gradient was used, the two were well-resolved. With the same slower gradient, 3.2 did not show any 19-mer failure sequence (supporting information). Because 19-mer failure sequence is most difficult to resolve from the full-length 20-mer sequence, it is conclusive that all failure sequences can be removed by the catching by polymerization purification technique. Another potential impurity in $\mathbf{3 . 2}$ may be the one from oxidation of dG to 8-oxo-dG, and this could not 
be detected by HPLC. We had described the synthesis of the same 20-mer sequence of 3.2 with one dG being replaced with 8-oxo-dG 5'-TCA TTG CT(8-oxo-dG) CTT AGA CCG CT-3' (3.4), and its digestion to nucleosides. We found that 8-oxo-dG could be detected by HPLC. In contrast, 8-oxo-dG could not be detected from nucleosides from digesting 3.2.[15] In addition, we had subjected the G nucleoside into the radical polymerization conditions and found that it was stable.[16] Here, we provide further evidence that $\mathrm{dG}$ is stable under radical acrylamide polymerzation conditions. When ODN 3.4, which contains 8-oxo-dG, was exposed to concentrated $\mathrm{NH}_{4} \mathrm{OH}$ at elevated temperature, multiple peaks were observed in HPLC profile (supporting information). This observation indicated that ODNs containing 8-oxo-dG is unstable under such conditions. Because our purification procedure involves in heating ODN in concentrated $\mathrm{NH}_{4} \mathrm{OH}$, and HPLC analysis showed single peak, we can conlude that ODNs purified with the catching by polymerization technology do not contain 8-oxo-dG.

It is interesting to note that radicals can damage DNA.[17] Then, why is ODN stable under the radical acrylamide polymerization conditions? To anwser this question, we need to consider the fact that DNA damage is mostly caused by the highly reactive hydroxyl radical. This radical is so reactive that it breaks carbonhydrogen bonds with little selectivity. The radical used to initiate the acrylamide polymerization reaction is the sulfate radical, which was reported to be 3,000 times less reactive than the hydroxyl radical.[18] In addition, under the polymerization conditions used for purification, large excess of acrylamide is used. This acrylamide could serve as a radical scavenger like vitamin E in the biological system. Once the 
sulfate radical is formed in the initiation stage of polymerization, it is quickly converted to the carbon radical of acrylamide. This radical is stabilized by the amide group through resonance, and may not be reactive enough to damage ODN, which requires abstraction of hydrogen atom from a carbon-hydrogen bond or breaking aromaticity of nucleoside bases by adding a radical to an aromatic ring.

For using the catching by polymerization method for ODN purification, one very important lesson we learned in the past few years is that fresh nucleoside phosphoramidite solutions need to be used for ODN synthesis. Otherwise, the purity of product may be lower. For example, to reduce costs, in some of our previous experiments, phosphoramidite solutions that were stored in a freezer under nitrogen in jars containing Drierite over half a year were used. The ODNs purified by catching by ploymerization were found contaminated with small amount of unidentifiable impurities.[15] After realizing this, we always used phosphoramidite solutions prepared within one week and found that ODN purity was consistently $100 \%$ after purification by polymerization. Leaving the solutions on the synthesizer for one week was found having no adverse effects on the purification results. It should be noted that the phosphoramidites do not need to be freshly purchased. In fact, the ones we used were usually stored in a freezer for more than six months.

The above refinements including using less acrylamide polymerization solution, using a centrifugal filter unit, and using fresher phosphoramidite solutions are critical for the catching by polymerization purification technology to be practically useful. The resulted improvements are summarized in Table 3.1. For example, in previous procedure, we used large excess of polymerization monomer. 
Therefore, we had to extract $0.25 \mu \mathrm{mol}$ ODN from more than $500 \mu \mathrm{L}$ gel.[14, 15] In the improved procedure, we only used $6 \mu \mathrm{L}$ monomer solution and the gel volume is less than $20 \mu \mathrm{L}$. Importantly, we observed supernatant over the gel, which may contain a large portion of ODN. In contrast, using previous procedure, there was only one phase. Due to the reduced gel volume and the existence of supernatant, the efficiency of ODN extraction is drastically improved. Previously, we had to be very careful to suck up the supernatant by pipette during extraction.[14, 15] It was inconvenient because gel fragments occasionally block pipette tip, and fine fragments can be sucked up, which contaminate ODN. With a centrifugal filter unit, extraction can be carried out by short spins.

\subsection{Conclusion}

In conclusion, several very important refinements of the catching failure sequences by polymerization ODN purification technology are presented. These refinements are critical for the technology to be practically useful. In addition, to demonstrate the purity of ODN, we synthesized a 19-mer failure sequence and proved that HPLC can resolve it from the 20-mer full-length sequence. The observation of ODN that contains 8 -oxo- $\mathrm{dG}$ is unstable under the purification conditions further confirmed that ODN is stable under the acrylamide radical polymerization conditions. The reasons for the stability are discussed. With these new results and findings, we are now very confident that the catching by polymerization purification method is highly convenient and highly reliable. We expect that it be widely used for small scale, large scale, and high throughput purification. 
Table 3.1. Summary of advantages of improved procedure over previous procedure

\begin{tabular}{|c|c|c|c|}
\hline Entry & Items & Previous procedure & Improved procedure \\
\hline 1 & $\begin{array}{l}\text { Polymerization } \\
\text { solution }\end{array}$ & $250 \mu \mathrm{L}$ & $\begin{array}{l}6 \mu \mathrm{L} \text {. Save monomers and reduce gel } \\
\text { volume }\end{array}$ \\
\hline 2 & ODN solution & $250 \mu \mathrm{L}$ & $50 \mu \mathrm{L}$ \\
\hline 3 & Gel volume & $\begin{array}{l}>500 \mu \mathrm{L} . \text { Larger volume makes } \\
\text { extraction less efficient }\end{array}$ & $\begin{array}{l}<20 \mu 1 . \text { Smaller volume makes } \\
\text { extraction more efficient }\end{array}$ \\
\hline 4 & Supernatant & $\begin{array}{l}\text { No. All ODN in gel, difficult to } \\
\text { extract }\end{array}$ & $\begin{array}{l}\text { Yes. Most ODN in supernatant, easier } \\
\text { to extract }\end{array}$ \\
\hline 5 & Water for extraction & $\begin{array}{l}12 \mathrm{~mL}(3 \mathrm{~mL} \times 4) . \text { Needs more time } \\
\text { to evaporate }\end{array}$ & $\begin{array}{l}0.15 \mathrm{~mL}(50 \mu \mathrm{L} \times 3) . \text { Needs less time } \\
\text { to evaporate }\end{array}$ \\
\hline 6 & Extraction time & $\begin{array}{l}12 \mathrm{~h}(3 \mathrm{~h} \times 4) . \text { Cannot finish in one } \\
\text { day }\end{array}$ & $30 \min (10 \min \times 3)$ \\
\hline 7 & Extraction method & $\begin{array}{l}\text { Pipetting supernatant. Difficult to } \\
\text { tip blockage by gel. Each extraction } \\
\text { takes } \sim 20 \text { min. Not suitable for } \\
\text { high throughput purification }\end{array}$ & $\begin{array}{l}\text { Spin. Each extraction takes } 10 \mathrm{sec} \text {. } \\
\text { Suitable for high throughput } \\
\text { purification }\end{array}$ \\
\hline 8 & Extract & $\begin{array}{l}\text { May contain gel, which needs } \\
\text { additional removal and reduces } \\
\text { recovery yield }\end{array}$ & No gel fragments, and very clean \\
\hline 9 & Recovery yield & $70-95 \%$ & $>95 \%$ \\
\hline 10 & ODN Purity & $92-100 \%$ & $\begin{array}{l}100 \% \text {. due to fresher } \\
\text { phosphoramidite solution }\end{array}$ \\
\hline
\end{tabular}

\section{Acknowledgements}

Financial support from US NSF (CHE-0647129 and CHE-1111192), MTU

Biotech Research Center, MUCI, and MTU REF-TC; the assistance from Mr. Dean W. Seppala (electronics) and Mr. Jerry L. Lutz (NMR); and NSF equipment grants (CH-1048655 and CHE-9512445) are gratefully acknowledged. 


\section{References and Notes}

1. Singh, Y., P. Murat, and E. Defrancq, Recent developments in oligonucleotide conjugation. Chemical Society Reviews, 2010, 39(6): p. 2054-2070.

2. Hughes, R.A., A.E. Miklos, and A.D. Ellington, Gene synthesis: methods and applications. Methods Enzymol, 2011, 498: p. 277-309.

3. Abramova, T., Frontiers and Approaches to Chemical Synthesis of Oligodeoxyribonucleotides. Molecules, 2013, 18(1): p. 1063-1075.

4. Kosuri, S. and G.M. Church, Large-scale de novo DNA synthesis: technologies and applications. Nature Methods, 2014, 11(5): p. 499-507.

5. Tian, J., K. Ma, and I. Saaem, Advancing high-throughput gene synthesis technology. Molecular BioSystems, 2009, 5(7): p. 714-722.

6. Ma, S., N. Tang, and J. Tian, DNA synthesis, assembly and applications in synthetic biology. Current opinion in chemical biology, 2012, 16(3): p. 260267.

7. Fang, S. and D.E. Bergstrom, Reversible biotinylation phosphoramidite for 5'end-labeling, phosphorylation, and affinity purification of synthetic oligonucleotides. Bioconjugate Chemistry, 2003, 14(1): p. 80-85.

8. Beller, C. and W. Bannwarth, Noncovalent Attachment of Nucleotides by Fluorous Fluorous Interactions: Application to a Simple Purification Principle for Synthetic DNA Fragments. Helvetica Chimica Acta, 2005, 88(1): p. 171-179. 
9. Mahajan, S., S. Patnaik, R. Gandhi, et al., Synthesis of labeled oligonucleotides through a new chemically cleavable linker. Tetrahedron Letters, 2005, 46(36): p. 6149-6153.

10. Fang, S. and D.E. Bergstrom, Fluoride-cleavable biotinylation phosphoramidite for 5'-end-labeling and affinity purification of synthetic oligonucleotides. Nucleic Acids Research, 2003, 31(2): p. 708-715.

11. Sproat, B.S., T. Rupp, N. Menhardt, et al., Fast and simple purification of chemically modified hammerhead ribozymes using a lipophilic capture tag. Nucleic Acids Research, 1999, 27(8): p. 1950-1955.

12. Pearson, W.H., D.A. Berry, P. Stoy, et al., Fluorous affinity purification of oligonucleotides. The Journal of Organic Chemistry, 2005, 70(18): p. 71147122.

13. Fang, S. and D.E. Bergstrom, Reversible 5'-end biotinylation and affinity purification of synthetic RNA. Tetrahedron Letters, 2004, 45(43): p. 79877990.

14. Fang, S., S. Fueangfung, X. Lin, et al., Synthetic oligodeoxynucleotide purification by polymerization of failure sequences. Chem. Commun., 2011, 47(4): p. 1345-1347.

15. Pokharel, D., Y. Yuan, S. Fueangfung, et al., Synthetic oligodeoxynucleotide purification by capping failure sequences with a methacrylamide phosphoramidite followed by polymerization. RSC Advances, 2014, 4(17): p. 8746-8757. 
16. Fang, S. and S. Fueangfung, Scalable Synthetic Oligodeoxynucleotide Purification with Use of a Catching by Polymerization, Washing, and Releasing Approach. Organic Letters, 2010, 12(16): p. 3720-3723.

17. Breen, A.P. and J.A. Murphy, Reactions of oxyl radicals with DNA. Free Radical Biology and Medicine, 1995, 18(6): p. 1033-1077.

18. Liang, C. and H.-W. Su, Identification of sulfate and hydroxyl radicals in thermally activated persulfate. Industrial \& Engineering Chemistry Research, 2009, 48(11): p. 5558-5562. 


\title{
Chapter 4
}

Methacrylation Phosphoramidite with Acid-Cleavable Linker for EcoFriendly Synthetic Oligodeoxynucleotide Purification ${ }^{1}$

\author{
Durga Pokharel, Shiyue Fang \\ Department of Chemistry, Michigan Technological University, 1400 Townsend Drive, \\ Houghton, MI 49931 USA \\ Email: shifang@mtu.edu
}

\footnotetext{
${ }^{1}$ The content of Chapter 4 will be submitted to a peer reviewed journal for publication.
} 
The idea of the project in chapter 4 was conceived by Dr. Shiyue Fang. All experiments in "Methacrylation Phosphoramidite with Acid-Cleavable Linker for Eco-Friendly Synthetic Oligodeoxynucleotide Purification" were carried out by Durga P. Pokharel. Dr. Fang revised writing carried out by Durga P. Pokharel.

\section{Apporved by advisor Dr. Shiyue Fang}




\begin{abstract}
A methacrylation phosphoramidite containing a linker cleavable with acetic acid was synthesized, and used for synthetic oligodeoxynucleotide (ODN) purification. During automated synthesis, the full-length ODN was tagged with the phosphoramidite. The failure sequences were not. In purification, the full-length ODN was co-polymerized into a polyacrylamide gel, and the failure sequences and other impurities were removed by washing. Pure ODN was cleaved from the gel with acetic acid. Using the method, purification of sequences as long as 151-mer and at scales as high as $50 \mu \mathrm{mol}$ was demonstrated. The products have high purity and good recovery yields. The method does not involve in any type of chromatography and purification is achieved through simple manipulations such as shaking and filtration. Compared with gel electrophoresis and HPLC purification methods, the new technology is less labor-demanding and more amendable for automation, consumes minimal amount of environmentally harmful organic solvents and requires little energy for solvent evaporation. Therefore, it is ideal for high throughput purification and large scale ODN-based drug purification as well as small scale purification.
\end{abstract}




\subsection{Introduction}

Synthetic ODNs have found wide applications.[1-5] Important recent examples include total gene synthesis for genome construction in synthetic biology projects,[6-11] and oligonucleotide-based drug development.[12-16] Gene synthesis requires hundreds of ODNs. In order for the synthetic genes to be affordable, the ODNs should be produced in a high throughput fashion. Due to automated ODN synthesis either using modern column-based synthesizers or microarray technologies, large numbers of ODNs can be synthesized simultaneously.[6-11] For gene assembly, it is crucial that the ODNs are highly pure. Currently, the ODNs are mostly purified using gel electrophoresis or HPLC. However, using these technologies purification in a highly parallel fashion is challenging, and high throughput ODN purification remains a bottle-neck for gene synthesis and synthetic genome construction. For oligonucleotide-based drug development, kilograms to metric tons of oligos are needed for clinical trials and patient use after the drugs are marketed. This requires large scale oligo production, which includes synthesis and purification. The challenges for large scale oligo synthesis have been largely overcome,[12, 13] but large scale purification remains a bottle-neck.[13-16] Currently, the most widely used technology for large scale purification of oligonucleotide-based drugs is reversedphase (RP) HPLC. The method is expensive to scale up, consumes large volumes of environmentally harmful organic solvents, and takes significant energy for solvent evaporation. The waste to product mass ratio can be as high as $10^{5}: 1$. 
In order to overcome the challenges currently existed in high throughput and large scale ODN purifications, we recently reported a new technology using the concept of catching by polymerization.[17-21] In one instance, during automated synthesis, the failure sequences were capped with acetic anhydride in each synthetic cycle, and the full-length sequences were tagged with a polymerizable methacrylamide group using a methacrylation phosphoramidite that contained a cleavable linker. At purification, the crude product was simply subjected to acrylamide polymerization conditions. The full-length sequences were copolymerized into the polyacrylamide gel, leaving the failure sequences and other impurities in solution, which were removed by washing. The full-length ODN was then cleaved from the polymer, and extracted with water. Highly pure ODN was obtained with excellent recovery yields.[18] The new purification technology does not involve any types of chromatography, and purification is achieved with simple manipulations such as shaking and filtration. The waste to product mass ratio can be as low as 1:1. As a result, the technology is highly suitable for high throughput and large scale purification. However, in our earlier studies, the linker we used in our methacrylation phosphoramidite for tagging full-length sequences was a diisopropylsilyl acetal function, and the highly toxic and corrosive hydrogen fluoride had to be used for cleaving the full-length sequences from the polyacrylamide gel. In addition, before cleavage the gel had to be dried under vacuum, which was energydemanding and time-consuming. Furthermore, the cleaving reaction was carried out in DMF, which is expensive for large scale purification and difficult to evaporate due to its high boiling point.[18] In this Paper, we report the synthesis of a new 95 
methacrylation phosphoramidite, which contains a linker that can be readily cleaved with the more environmentally benign $80 \%$ acetic acid, and its application in ODN purification using the catching full-length sequences by polymerization approach. We demonstrate that even for purification of sequences as long as 151-mer and at scales as high as $50 \mu \mathrm{mol}$, the purification procedure requires little modification, and the technology is still highly efficient.

\subsection{Results and Discussion}

\section{Synthesis of Methacrylation Phosphoramidite 1}

The phosphoramidite (4.1), which contains a polymerizable methacrylamide group and an acid-labile alkyl trityl ether linker, was synthesized according to Scheme 4.1. The commercially available 4-hydroxybenzophenone (4.2) was reacted with methyl 6-bromohexanoate in the presence of potassium carbonate to give $\mathbf{4 . 3}$. Compound 4.3 was treated with 4-methoxyphenylmagnesium bromide to give $\mathbf{4 . 4}$, which was conveniently converted to 4.5 by simply heating with $2,2^{\prime}$ (ethylenedioxy)bis(ethylamine) in water. Under these conditions, mono-acylation was favored over di-acylation, which is a common side reaction for mono-acylation of symmetric diamines.[22, 23] Compound 4.5 was acylated with methacrolyl chloride using DIEA as base to give 4.6. Converting 4.6 to 4.7 was achieved by first treating 4.6 with excess acetyl chloride. After removing volatiles, without purification, the intermediate trityl chloride was used directly to react with thymidine in pyridine. Product 4.7 was obtained in 77\% isolated yield.[24] Phosphinylation of 4.7 using 2- 
cyanoethyl $N, N, N^{\prime}, N^{\prime}$-tetraisopropylphosphordiamidite with diisopropylammonium tetrazolide as activator gave compound $\mathbf{4 . 1}$ in $87 \%$ yield. All the reactions are simple, and the reagents are inexpensive or can be replaced with cheaper ones. We expect that the synthesis could be easily scaled up to kilograms.
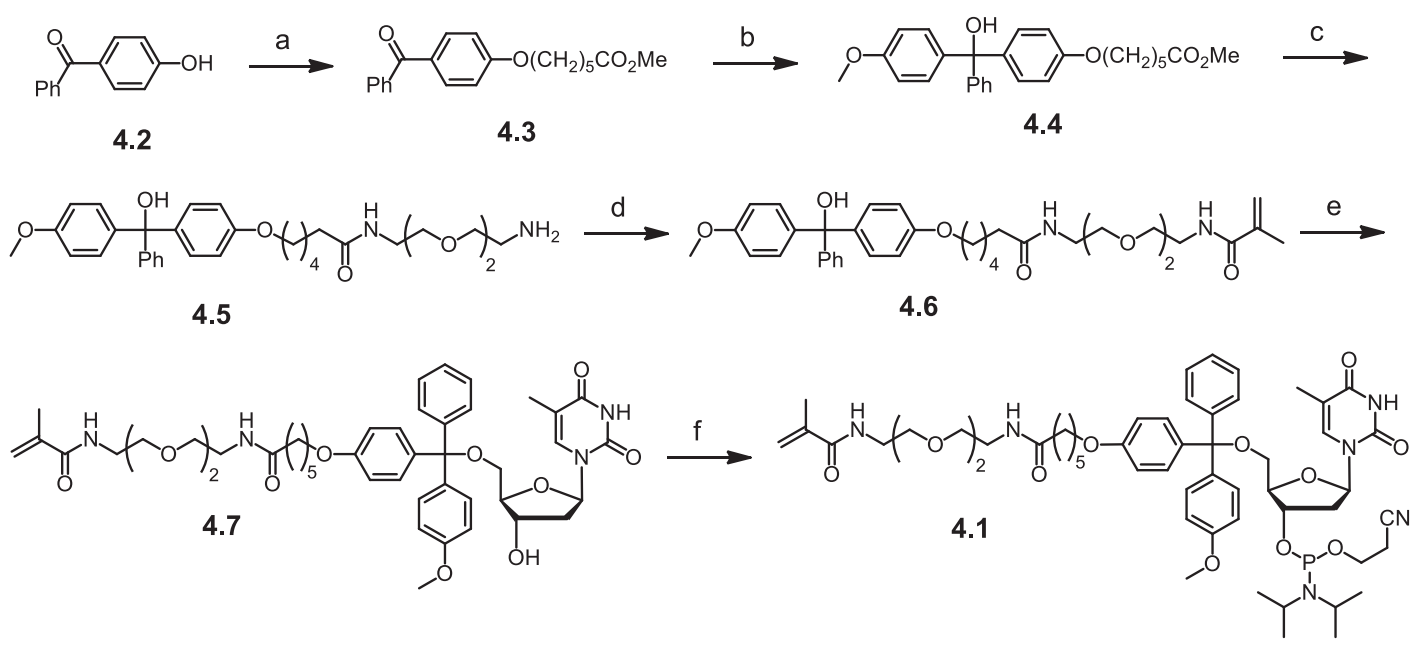

Scheme 4.1. Synthesis of methacrylation phosphoramidite 1. Reagents and conditions: (a) $\mathrm{Br}\left(\mathrm{CH}_{2}\right)_{5} \mathrm{CO}_{2} \mathrm{CH}_{3}$ (1.0 equiv), $\mathrm{K}_{2} \mathrm{CO}_{3}$ (4.0 equiv), acetone, reflux, 12 h, $85 \%$; (b) $p-\mathrm{H}_{3} \mathrm{COPhMgBr}(3.0),-78{ }^{\circ} \mathrm{C}$ to rt, $4 \mathrm{~h}, 56 \%$; (c) $\left[\mathrm{CH}_{2} \mathrm{O}\left(\mathrm{CH}_{2}\right)_{2} \mathrm{NH}_{2}\right]_{2}(3.0$ equiv), $\mathrm{H}_{2} \mathrm{O}, 85 \mathrm{C}, 12 \mathrm{~h}, 75 \%$; (d) methacrolyl chloride (1.0 equiv), DIEA (3.0 equiv), $\mathrm{CH}_{2} \mathrm{Cl}_{2}, 0{ }^{\circ} \mathrm{C}$ to rt, $12 \mathrm{~h}, 88 \%$; (e) acetyl chloride, $\mathrm{rt}, 2 \mathrm{~h}$; then thymidine (1.0 equiv), pyridine, rt, $18 \mathrm{~h}, 77 \%$; (f) $\left(\mathrm{iPr}_{2} \mathrm{~N}\right)_{2} \mathrm{PO}\left(\mathrm{CH}_{2}\right)_{2} \mathrm{CN}$ (1.1 equiv), diisopropylammonium tetrazolide (1.0 equiv), rt, $5 \mathrm{~h}, 87 \%$. 


\section{Purification at Small Scales}

Table 4.1. ODN sequences

\begin{tabular}{lll}
\hline ODN & Length & \multicolumn{1}{c}{ Sequences } \\
\hline $\mathbf{4 . 8}$ & 20-mer & 5'-T CAT TGC TGCT TAG ACC GCT-3' \\
$\mathbf{4 . 9}$ & 31-mer & 5'-T CGG ATT ATG TCG ATA TTA GGA GAA TGG TAT-3' \\
$\mathbf{4 . 1 0}$ & 37-mer & 5'-T ATA CCA TTC TCC TAA TAT CGA CAT AAT CCG TCG ATC-3' \\
$\mathbf{4 . 1 1}$ & 61-mer & 5'-T AAA GCT ATA GGT ACA GTA TTA GTA GGA CCT ACA CCT \\
& & GTC AAG ATA ATG GTC CAG GTC GGT-3' \\
$\mathbf{4 . 1 2}$ & 151-mer & 5'-T CAA CAA AAT CAT TTT GCA CCA TGT GGA GCA CCT CCA \\
& & AAT AAC ACC TTT ATA ACC CAT GTG GCG TAA TCA TTG TTT \\
& & TCC ATC CTA GAA AGC TCA TAC AAT GCG TTT TTC ATG AGT \\
& & TTA TTT TCA TGC TCT AGT TTA GTC ATC TTC TTT TCT-3' \\
\hline
\end{tabular}

The 20-mer ODN sequence $\mathbf{4 . 8}$ (see Table 4.1 for ODN sequences) was used to demonstrate the convenience of the new technology for the purification of short ODNs on small scales. The ODN was synthesized on a $1 \mu \mathrm{mol}$ scale under standard conditions using phosphoramidite chemistry. The synthesizer manufacturer suggested cycle was slightly modified so that the methacrylation phosphoramidite 4.1 could be coupled to the 5'-end of full-length sequences on the synthesizer automatically (Figure 4.1). If one does not prefer to spend time on cycle modification, manual coupling can also be used. For automated coupling, the standard cycle was copied to a new file. After the coupling steps and before the capping steps, a 180 second waiting step was inserted, and was set to be active only for base 5. The solution of $\mathbf{4 . 1}$ in acetonitrile at the same concentration $(0.1 \mathrm{M})$ as other standard phosphoramidite monomers was attached to this bottle position. In the sequence, the nucleotide at the 5 '-end was edited to be 5 , which in the example here incorporated the nucleotide dT while tagging the ODN with a methacrylamide group. Except for these modifications, 
all other synthesis, deprotection and cleavage conditions were standard. Briefly, CPG with a succinyl ester linker was used as solid support. The phosphoramidite monomers were 5'-DMTr 2-cyanoethyl benzoyl-dA, acetyl-dC, isobutyryl-dG, and dT. Their $0.1 \mathrm{M}$ solutions in acetonitrile were used for coupling with DCI as the activator. Failure sequences were capped with acetic anhydride in each synthetic cycle. At the end of synthesis, detritylation was not carried out as this would remove the methacrylamide tag. Cleavage was carried out on the synthesizer with concentrated ammonium hydroxide. Deprotection was achieved by heating the ammonium hydroxide solution to $55{ }^{\circ} \mathrm{C}$ for 15 hours. After adding $\sim 200 \mu \mathrm{L}$ DIEA, the solutions were divided into five equal portions, and evaporated to dryness under vacuum. One portion was dissolved in $300 \mu \mathrm{L}$ water, and $20 \mu \mathrm{L}$ was injected into RP HPLC to give trace a (Figure 4.2). 
A
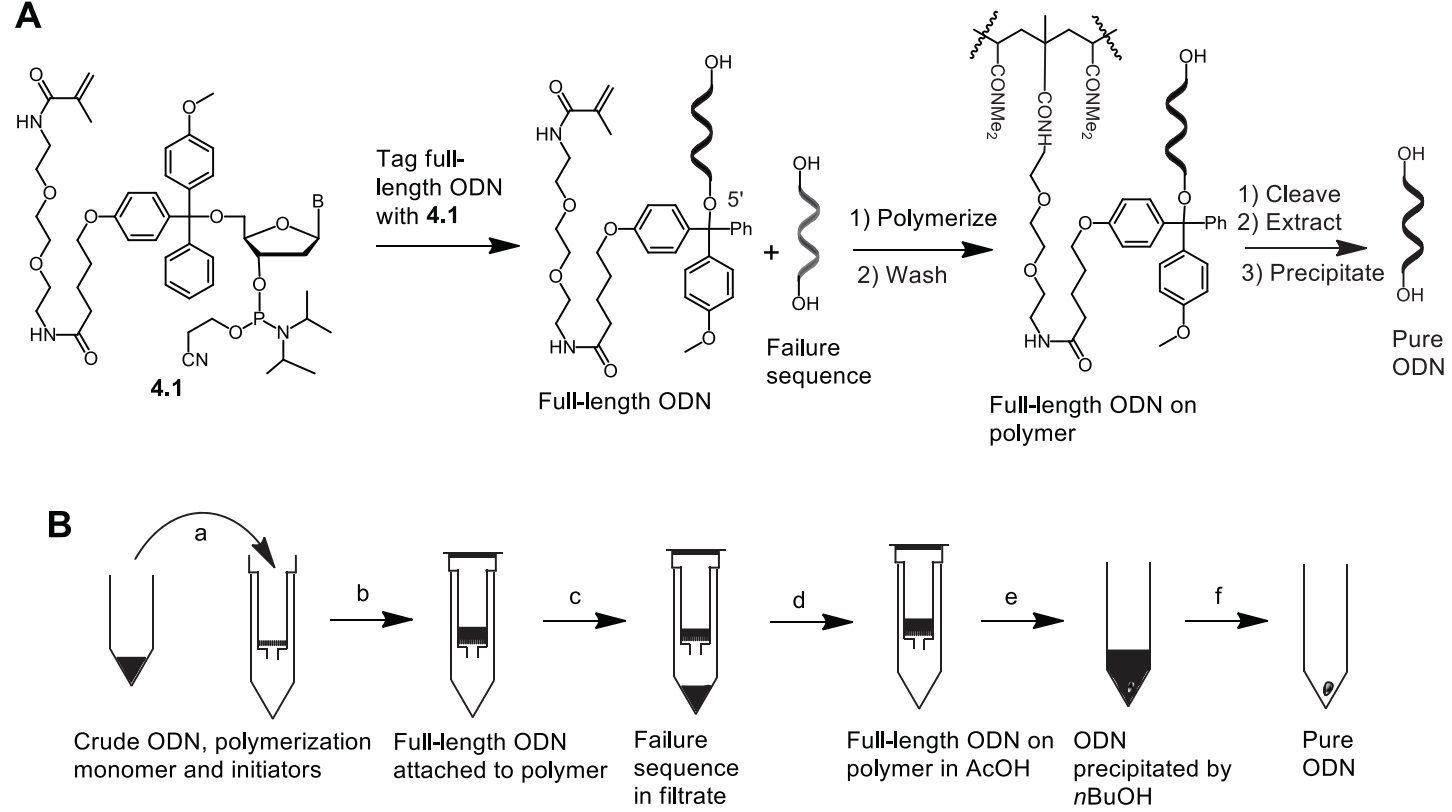

Figure 4.1. The catching full-length sequences by polymerization ODN purification technology. (A) Principle. (B) Workflow: (a) Add polymerization monomer and initiators to crude ODN, and transfer to a centrifugal filter unit. (b) Wait for polymerization. (c) Spin, add water, and spin. (d) Add 80\% AcOH. (e) Spin, add water, spin, evaporate filtrate, and precipitate with $n \mathrm{BuOH}$. (f) Remove supernatant.

Once the full-length sequences were tagged with a methacrylamide group, purification was very simple (Figure 4.1). To the second portion of the crude ODN $(\sim 0.2 \mu \mathrm{mol})$, small amounts of water $(50 \mu \mathrm{L})$, polymerization solution containing $N, N$-dimethylacrylamide and $N, N^{\prime}$-methylenebis(acrylamide) $(12 \mu \mathrm{L}$, see supporting information for recipe), 5\% ammonium peroxide $(5 \mu \mathrm{L})$ and $0.66 \mathrm{M}$ TMEDA solutions $(5 \mu \mathrm{L})$ were added sequentially. For convenient filtration during subsequent washing, cleavage and extraction, before polymerization the solution was transferred 
into the top compartment of a centrifugal filtering unit using a pipette. The solution could stay above the filter disc even without stopping the bottom of the compartment. The polymerization occurred quickly within 15 minutes, but it was allowed to proceed for 1 hour to ensure completion (Figure 4.1). The full-length sequences were covalently linked to polymer, and failure sequences remained in solution. The polyacrylamide gel was loosened with a clean spatula, and water containing 5\% $\mathrm{Et}_{3} \mathrm{~N}$ was added to wash away impurities including failure sequences and small molecules. The wash was very convenient because the supernatant could be removed by a simple spin. So, it was carried out for six times. A final wash with pure water was performed to rinse away residue $\mathrm{Et}_{3} \mathrm{~N}$. To cleave the full-length sequences from the polymer, minimum amount of $80 \%$ acetic acid that could cover the gel $(120 \mu \mathrm{L})$ was added, and the mixture was incubated for 5 minutes. The supernatant was removed by spin, and the cleavage was repeated for two times. To extract remaining ODN, minimum amount of water that could cover the gel $(120 \mu \mathrm{L})$ was added, and the supernatant was collected by spin. This was repeated for four times. The acetic acid and water extracts were combined and evaporated to dryness. The residue was dissolved in 100 $\mu \mathrm{L}$ concentrated ammonium hydroxide and precipitated with $900 \mu \mathrm{l} n \mathrm{BuOH}$. Pure ODN 4.8 was dissolved in $300 \mu \mathrm{L}$, and $20 \mu \mathrm{L}$ was injected into RP HPLC to give trace $\mathrm{b}$ (Figure 4.2). As shown, the ODN was $100 \%$ pure. Because the theoretical amount of ODN for generating traces a and $\mathrm{b}$ were equal, the recovery yield of the purification process was easily determined to be $68 \%$ by dividing the area of the peak in trace $b$ at 19 minutes by that in trace a at 45 minutes. 
To demonstrate the simplicity and effectiveness of the purification procedure with more examples, the slightly longer ODNs 4.9 (31-mer) and 4.10 (37-mer) were synthesized and purified. ODN 4.9 was synthesized at $1 \mu \mathrm{mol}$ scale under the same conditions described for $\mathbf{4 . 8}$, and $\mathbf{4 . 1 0}$ was synthesized at $10 \mu \mathrm{mol}$ scale described in the section of large scale purification. For cleavage and deprotection, we tested different conditions. The mixture of concentrated ammonium hydroxide and methylamine solutions at 1:1 volume ratio was used in both cases. This reagent cleaved the ODN from CPG and removed all protecting groups at room temperature in three hours, and therefore the procedure was more convenient. The purification procedures were similar to that for purifying $\mathbf{4 . 8}$ with only slight modifications (see experimental section). Both ODNs were obtained in $100 \%$ purity, and the recovery yields were high. RP HPLC profiles are in supporting information. 

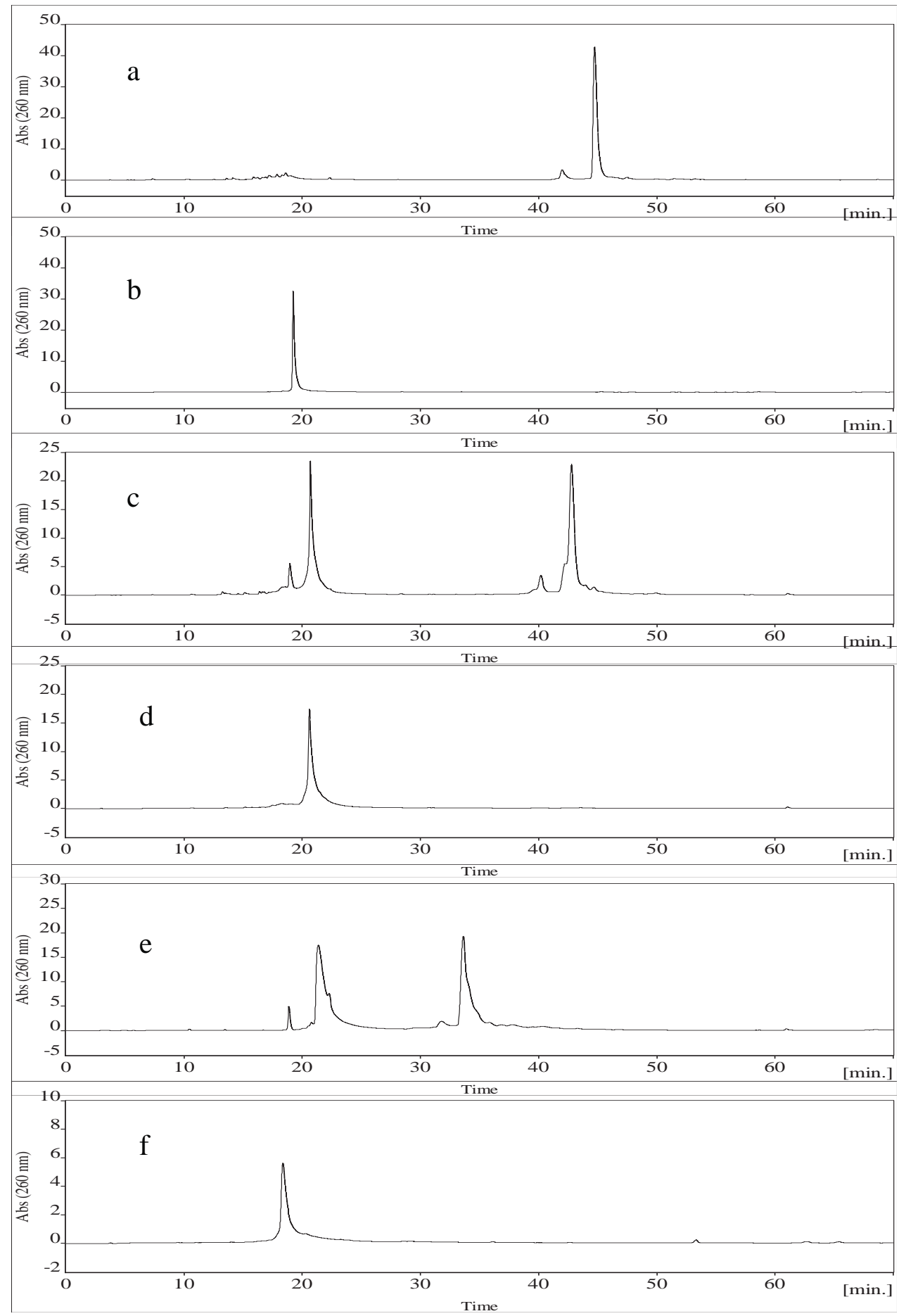

Figure 4.2. RP HPLC profiles of ODNs; (a) crude 4.8 (b) pure 4.8 (c) crude 4.10 (d) pure 4.10 (e) crude $\mathbf{4 . 1 2}$ (f) pure $\mathbf{4 . 1 2}$ 


\section{Purification at Larger Scales}

A major application of the technique is the purification of ODNs on large scales, which is important for large scale ODN-based drug production. To demonstrate the suitability of our method for this purpose, we decided to carry out a purification on a $50 \mu \mathrm{mol}$ scale using ODN 4.10 as the example. The ODN was synthesized in five $10 \mu \mathrm{mol}$ columns with $1000 \AA \mathrm{CPG}$. The same procedure and reagents for synthesizing $\mathbf{4 . 8}$ were used except that the synthesis cycle was modified from $10 \mu \mathrm{mol}$ cycle. According to trityl essay before the cycle for attaching $\mathbf{4 . 1}$, the average yield of 36-mer for the five synthesis was 50\%. Cleavage and deprotection were achieved using the mixture of concentrated ammonium hydroxide and methylamine. RP HPLC analysis of the crude indicated that only about $50 \%$ of the 36-mer intermediate was successfully methacrylated with 4.1 (trace c, Figure 4.2). As a result, the synthesis yield of the 37 -mer was $25 \%$. In the future, the synthesis should be improved by increasing coupling time and capping time, and by coupling 4.1 for one more time or allowing it to proceed longer. These modifications are expected to give higher synthesis yield. For purification, the crude ODN was dissolved in 1.25 $\mathrm{mL}$ water in a $25 \mathrm{~mL}$ round-bottomed flask, and polymerization was carried out using as little as $1.25 \mathrm{~mL}$ polymerization solution. The gel was transferred into a $15 \mathrm{~mL}$ Büchner funnel with a sintered glass disc and broken into several pieces with a spatula. Washing failure sequences and other impurities were simply achieved using water containing $\mathrm{Et}_{3} \mathrm{~N}$ followed by water. The wash was convenient because the solutions could be easily removed by applying vacuum. Cleaving the full-length 
sequences from gel was achieved under the same conditions in small scale purifications with only a slightly larger volume of $80 \% \mathrm{AcOH}(\sim 4 \mathrm{~mL})$. Extraction of full-length sequences and precipitation with $n \mathrm{BuOH}$ were also very convenient (see experimental section). Pure ODN 4.10 was obtained as nice white cotton-like fluffy fibers (see picture in supporting information). The weight was $98 \mathrm{mg}$, which corresponds to a $70 \%$ recovery yield of the purification process. RP HPLC analysis showed that the ODN was $100 \%$ pure (trace d, Figure 4.2). The images ${ }^{1} \mathrm{H}$ NMR are in supporting information.

\section{Long Sequence Purification}

Another important application of the technique is long ODN purification, which is especially important for total gene synthesis. Because long ODNs can usually assume secondary structures and the hydrophobicity of DMTr group becomes less distinctive as the length increases, they are challenging to purify with other methods including trityl-on RP HPLC. Because our catching by polymerization technology attaches the full-length sequences to a polymer gel covalently, in principle, there is no limit on the length of ODN that can be purified using the method. To demonstrate the power of the technique, the 61-mer 4.11 and the 151-mer 4.12 were synthesized and purified. ODN 4.11 was synthesized on $1 \mu \mathrm{mol}$ scale using the conditions for 4.9 on $1000 \AA$ CPG. ODNs 4.12 was synthesized on $0.2 \mu$ mol scale using $2000 \AA$ A CPG. To increase recovery yield and capping efficiency, additional coupling and capping times were added in the synthetic cycle (see experimental section). In addition, before the synthesis, the CPG was subjected to the capping 
conditions for 20 minutes. All other conditions were the same as for the synthesis of 4.8 (see experimental section). The synthesis yields as indicated by trityl assay before the last cycle for coupling 4.1 were $83 \%$ (for 4.11) and 22\% (for 4.12). Cleavage and deprotection were achieved using the mixture of concentrated ammonium hydroxide and methylamine. Purification was carried out at $0.2 \mu \mathrm{mol}$ (for 4.11) and $0.04 \mu \mathrm{mol}$ (for 4.12) scales. Polymerization was achieved under the same conditions described for 4.8 using the same amount of polymerization reagents even though the purification scales and sequence lengths were different. For washing failure sequences from gel, the same conditions for 4.9 were used for 4.11. For the longer ODNs 4.12, 3.0 M NaOAc was used to increase washing efficiency. The residue $\mathrm{NaOAc}$ was removed by washing with $1 \% \mathrm{Et}_{3} \mathrm{~N}$ and water sequentially. Cleavage of ODN from gel, extraction and $n \mathrm{BuOH}$ precipitation were carried out under the same conditions described for 4.8. Recovery yields were determined to be $83 \%$ (for $\mathbf{4 . 1 1}$ ) and $50 \%$ (for 4.12) by comparing areas of pure and crude HPLC peaks. All ODNs were $100 \%$ pure. The crude and pure HPLC profiles for $\mathbf{4 . 1 2}$ are shown in Figure 4.2. Those for 4.11 are in supporting information.

\section{Reagent Mass Considerations}

A major advantage of the catching by polymerization purification technology compared to the most widely used RP HPLC is the low waste to product ratio. In the above purification processes, for small scale purifications including $0.2 \mu \mathrm{mol}$ scale purification of 20-mer 4.8, $0.2 \mu \mathrm{mol}$ scale purification of 31-mer 4.9, $0.2 \mu \mathrm{mol}$ scale purification of 61-mer 4.11, and $0.04 \mu \mathrm{mol}$ scale purification of 151-mer 4.12, we 
consistently used $12 \mu \mathrm{l}$ polymerization solution [3.7 M N,N-dimethylacrylamide and $0.37 \mathrm{M} N, N^{\prime}$-methylenebis(acrylamide)]. The molar ratio of polymerization monomers [moles of $N, N$-dimethylacrylamide and $N, N^{\prime}$-methylenebis(acrylamide)] over the nucleotides in the ODNs (moles of ODN times the number of nucleosides) were 12.2 (for $0.2 \mu \mathrm{mol} \mathrm{4.8),} 7.9$ (for $0.2 \mu \mathrm{mol}$ 4.9), 4.0 (for $0.2 \mu \mathrm{mol}$ 4.11), and 8.1 (for $0.04 \mu \mathrm{mol} \mathrm{4.12}$ ). For the $1 \mu \mathrm{mol}$ scale purification of 37-mer 4.10, $24 \mu \mathrm{l}$ polymerization solution was used, the molar ratio was 2.6 . For the $50 \mu \mathrm{mol}$ purification of 37-mer 4.10, $1.25 \mathrm{~mL}$ polymerization solution was used, and the molar ratio was 2.8. Our guideline is that the molar ratio of polymerization monomer over nucleotides in the ODN should be equal to or slightly higher than 2.5. If we assume that an ODN contains equal numbers of the four nucleotides, the mass ratio of polymerization monomers over ODN is around 0.83 , which means that purification of one gram of ODN requires less than one gram of polymerization monomers. Molar ratio lower than 2.5 is not suggested because the full-length sequences may not be caught completely and the polymerization may not be efficient due to diluted polymerization monomer concentration. For small scale purification of short sequences, when the molar ratio of 2.5 is used, the volume of polymerization solution may be too tiny for convenient manipulation. As a result, a larger ratio is suggested. In our examples, $12 \mu \mathrm{L}$ polymerization solution was consistently used in the purification of $4.8(0.2 \mu \mathrm{mol}), 4.9(0.2 \mu \mathrm{mol}), 4.11(0.2 \mu \mathrm{mol})$, and $4.12(0.04 \mu \mathrm{mol})$. For larger scale purification, molar ratio much higher than 2.5 is not recommended even though the polymerization monomers are inexpensive. With large amount of gel, 
extraction of ODN after washing failure sequences requires relatively more water, which consumes energy for evaporation.

Besides the mass ratio of polymerization monomers over ODN, there are other mass ratios that need to be decided although these ratios are not critical for the success of purification. In all the above purification examples, the molar ratio of $\mathrm{N}, \mathrm{N}$ dimethylacrylamide over $N, N^{\prime}$-methylenebis(acrylamide) was set to be 10:1. Other ratios could also be used, but ratios higher than $40: 1$ give gels that are too soft for convenient handling and ratios lower than 5:1 may reduce ODN extraction efficiency. For the volume of water used to dissolve ODN before adding polymerization solution, it can also be varied but should not be too far away from our examples. The ones we used ranged from one $(50 \mu \mathrm{mol}$ purification of 4.10) to four $(0.2 \mu \mathrm{mol}$ purification of 4.8) times of that of polymerization solution. The initiators are usually more than enough even though only $5 \mu \mathrm{l}$ of each were used in most of our examples. For the $50 \mu \mathrm{mol}$ purification of $\mathbf{4 . 1 0}$, we used $10 \mu \mathrm{l}$ of each to ensure efficient polymerization. The molar ratio of TMEDA over ammonium persulfate was set to be 3:1 in our examples, but this is not a requirement. For washing failure sequences from gel, we used water containing bases such as $\mathrm{Et}_{3} \mathrm{~N}$ and, $\mathrm{NaOAc}$ followed by pure water. Adding bases can ensure the stability of the trityl alkyl ether linkage for anchoring full-length sequences to gel and is expected to increase washing efficiency. The volume of the washing solutions can be large because the solutions are inexpensive and not harmful to environment, and do not require evaporation. For cleaving and extraction full-length sequences from gel, minimum amount of $80 \%$ 
$\mathrm{AcOH}$ and water that can cover gel should be used. Efficiency should not be achieved using large amount of solutions, instead it should be achieved through repetitions. Minimizing the total volume is important for saving time and energy for evaporation.

\subsection{Experimental Section}

\section{General Procedures}

All reagents and solvents were used from commercial sources as received unless otherwise indicated. Dry THF and $\mathrm{CH}_{2} \mathrm{Cl}_{2}$ were from Innovative Technology's PureSolv ${ }^{\mathrm{TM}}$ system. Dry pyridine was obtained from distillation over $\mathrm{CaH}_{2}$ under nitrogen. All reactions were carried in oven-dried glassware under nitrogen. ${ }^{1} \mathrm{H},{ }^{13} \mathrm{C}$ and ${ }^{31} \mathrm{P}$ NMR spectra were measured on a Varian UNITY INOVA spectrometer at 400, 100 and $162 \mathrm{MHz}$, respectively. High resolution mass spectra were obtained on an Agilent Q-TOF system using a dual electrospray ionization source. MALDI-TOF mass spectrometry experiments were performed on Waters' Tofspec-2E. ODNs were synthesized on an ABI 394 solid phase synthesizer. RP HPLC was carried out using the JASCO LC-2000Plus System with the pump PU-2089Plus Quaternary Gradient;

detector UV-2075Plus; column C-18 analytical (5 $\mu$ m diameter, $100 \AA$ A, $250 \times 4.6$ $\mathrm{mm}$ ); solvent A, $0.1 \mathrm{M}$ triethylammonium acetate, 5\% acetonitrile; and solvent $\mathrm{B}, 90$ $\%$ acetonitrile. Profiles were generated by detection of absorbance of ODN at $260 \mathrm{~nm}$ using the gradient system, solvent B (0\%-45\%) in solvent A over 60 min followed by solvent B (45\%-100\%) in solvent A over $20 \mathrm{~min}$ at a flow rate of $1 \mathrm{~mL} / \mathrm{min}$ unless otherwise noted. In all purification examples, the solution that contains $3.7 \mathrm{M} \mathrm{N}, N$ -

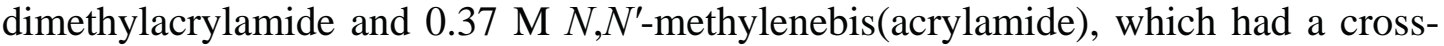
109 
linking ratio of 10:1, was used as the polymerization solution for catching full-length sequences. Solutions of $5 \%\left(\mathrm{NH}_{4}\right)_{2} \mathrm{~S}_{2} \mathrm{O}_{8}$ and $0.66 \mathrm{M}$ TMEDA were used as polymerization initiators.

\section{Compound 4.3}

Compound 4.2 (5.0 g, 25.24 mmol, 1.0 equiv), methyl 6-bromohexanoate (4.1 $\mathrm{mL}, 25.24 \mathrm{mmol}, 1.0$ equiv) and $\mathrm{K}_{2} \mathrm{CO}_{3}(14.0 \mathrm{~g}, 100.96 \mathrm{mmol}, 4.0$ equiv) was charged to a round-bottomed flask. About $80 \mathrm{~mL}$ of acetone was added which was previously passed through anhydrous sodium sulfate. The mixture was refluxed for overnight. After cooling to rt, the mixture was concentrated under reduced pressure to remove acetone. The remaining contents were transferred into a separatory funnel and partitioned between ethyl acetate and water. The organic phase was dried over anhydrous $\mathrm{Na}_{2} \mathrm{SO}_{4}$, filtered and the filtrate was concentrated to dryness under reduced pressure and the residue was recrystallized between ethyl acetate and hexane giving pure 4.3 as a white solid. (7.0 g, $21.44 \mathrm{mmol}, 85 \%): R_{\mathrm{f}}=0.5$ (Hexane/EtOAc, 3:1); ${ }^{1} \mathrm{H}$ NMR $\left(400 \mathrm{MHz}, \mathrm{CDCl}_{3}\right) \delta$ 7.80-7.71 (m, 4H), 7.56-7.51 (m, 1H) 7.47-7.43 (m, 2H), 6.93-6.90 (m, 2H), $4.02(\mathrm{t}, \mathrm{J}=7.2 \mathrm{~Hz}, 2 \mathrm{H}), 3.66(\mathrm{~s}, 3 \mathrm{H}), 2.34(\mathrm{t}, \mathrm{J}=7.2 \mathrm{~Hz} 2 \mathrm{H})$, 1.86-1.79 (m, 2H), 1.74-1.66 (m, 2H), 1.55-1.49 (m, 2H); ${ }^{13} \mathrm{C}$ NMR (100 MHz, $\left.\mathrm{CDCl}_{3}\right) \delta 195.7,174.1,162.9,138.5,132.8,132.0,130.2,129.9,128.4,114.2,68.1$, 51.7, 34.1, 29.0, 25.8, 24.8. 


\section{Compound 4.4}

Compound 4.3 (5.0 g, $15.31 \mathrm{mmol}, 1.0$ equiv) in a round-bottomed flask was stored over Drierite under vacuum in a dessicator overnight. The flask was then connected to a nitrogen atmosphere via a Schlenk manifold. Freshly distilled THF (60 $\mathrm{mL}$ ) was added. After cooling to $-78^{\circ} \mathrm{C}$, the solution of 4-methoxyphenymagnesium bromide in THF (0.5 M, $92 \mathrm{~mL}, 45.95 \mathrm{mmol}, 3.0$ equiv) was added slowly via a syringe. After addition, the mixture was allowed to warm up to $\mathrm{rt}$ gradually. After a total of $4 \mathrm{~h}$, the reaction was quenched with water, and THF was evaporated under reduced pressure. The remaining contents were transferred into a separatory funnel and partitioned between ethyl acetate and $5 \% \mathrm{NaHCO}_{3}$. The organic phase was dried over anhydrous $\mathrm{Na}_{2} \mathrm{SO}_{4}$, filtered and the filtrate was concentrated to dryness under reduced pressure. The product was purified with flash column chromatography $\left(\mathrm{SiO}_{2}\right.$, hexanes/EtOAc/Et $3 \mathrm{~N}, 3: 1: 0.2)$ giving 4.4 as a thick oil $(3.7 \mathrm{~g}, 8.52 \mathrm{mmol}, 56 \%): R_{\mathrm{f}}=$ 0.4 (hexanes/EtOAc/Et $3 \mathrm{~N}, 3: 1: 0.4) ;{ }^{1} \mathrm{H} \mathrm{NMR} \quad\left(400 \mathrm{MHz}, \mathrm{CDCl}_{3}\right) \delta 7.30-7.15(\mathrm{~m}$, 9H), 6.84-6.40 (m, 4H) $3.91(\mathrm{t}, J=6.4 \mathrm{~Hz} 2 \mathrm{H}), 3.71(\mathrm{~s}, 3 \mathrm{H}), 3.61(\mathrm{~s}, 3 \mathrm{H}), 3.49$ (br s, 1H), $2.30(\mathrm{t}, J=7.2 \mathrm{~Hz} 2 \mathrm{H}), 1.78-1.63(\mathrm{~m}, 4 \mathrm{H}), 1.51-1.46(\mathrm{~m}, 2 \mathrm{H}) ;{ }^{13} \mathrm{C}$ NMR (100 $\left.\mathrm{MHz}, \mathrm{CDCl}_{3}\right) \delta 174.3,158.8,158.3,147.9$ 139.9, 139.8, 129.5, 128.1, 128.0, 127.2, 113.9, 113.3, 81.6, 67.8, 55.4, 51.7, 34.1, 29.2, 25.9, 24.9; HRMS (ESI, [M+Na] $\left.{ }^{+}\right)$ $m / z$ calcd for $\mathrm{C}_{27} \mathrm{H}_{30} \mathrm{NaO}_{5}$ 457.1991, found 457.1989.

\section{Compound 4.5}

Compound $\quad 4.4 \quad(3.0 \quad \mathrm{~g}, \quad 6.91 \quad \mathrm{mmol}, \quad 1.0 \quad$ equiv $), \quad 2,2^{\prime}-$ (ethylenedioxy)bis(ethylamine) $(3.1 \mathrm{~mL}, 20.73 \mathrm{mmol}, 3.0$ equiv) and water $(700 \mu \mathrm{L})$ 
was charged to a round-bottomed flask. The mixture was stirred under nitrogen at 85 ${ }^{\circ} \mathrm{C}$ overnight. After cooling to $\mathrm{rt}$, the mixture was concentrated and the residue was purified by flash column chromatography $\left(\mathrm{SiO}_{2}, \mathrm{Et}_{2} \mathrm{O} / \mathrm{MeOH} / \mathrm{Et}_{3} \mathrm{~N}, 3: 2: 0.2\right)$ giving 5 as a thick oil $(2.85 \mathrm{~g}, 5.18 \mathrm{mmol}, 75 \%): R_{\mathrm{f}}=0.5\left(\mathrm{Et}_{2} \mathrm{O} / \mathrm{MeCN} / \mathrm{MeOH} / \mathrm{Et}_{3} \mathrm{~N}, 5: 2: 2: 1\right)$; ${ }^{1} \mathrm{H}$ NMR $\left(400 \mathrm{MHz}, \mathrm{CDCl}_{3}\right) \delta$ 7.24-7.08 (m, 9H), 6.80-6.70 (m, 4H) 6.39 (br s, $\left.1 \mathrm{H}\right)$, $3.86(\mathrm{t}, J=6.4 \mathrm{~Hz} 2 \mathrm{H}), 3.71(\mathrm{~s}, 3 \mathrm{H}), 3.53-3.46(\mathrm{~m}, 6 \mathrm{H}), 3.39-3.34(\mathrm{~m}, 4 \mathrm{H}), 2.75-2.65$ (m, 4H), $2.10(\mathrm{t}, J=7.2 \mathrm{~Hz} 2 \mathrm{H}), 1.74-1.58(\mathrm{~m}, 4 \mathrm{H}), 1.44-1.37(\mathrm{~m}, 2 \mathrm{H}) ;{ }^{13} \mathrm{C} \mathrm{NMR}$ $\left(100 \mathrm{MHz}, \mathrm{CDCl}_{3}\right) \delta 173.3,158.6,158.1,147.9$ 140.1, 139.9, 128.4, 128.0, 127.9, $126.9,113.7,113.2,81.3,72.8,70.3,70.2,70.1,67.8,55.4,41.5,39.3,36.5,29.2$, 25.9, 25.6, HRMS (ESI, $[\mathrm{M}+\mathrm{H}]^{+}$) $\mathrm{m} / z$ calcd for $\mathrm{C}_{32} \mathrm{H}_{43} \mathrm{~N}_{2} \mathrm{O}_{6}$ 551.3121, found 551.3126.

\section{Compound 4.6}

To an oven-dried round-bottomed flask under nitrogen was added 4.5 (2.7 g, $4.91 \mathrm{mmol}, 1.0$ equiv), dry $\mathrm{CH}_{2} \mathrm{Cl}_{2}(40 \mathrm{~mL})$ and DIEA (2.6 mL, $14.73 \mathrm{mmol}, 3.0$ equiv). After cooling to $0{ }^{\circ} \mathrm{C}$, methacrolyl chloride $(479 \mu \mathrm{L}, 4.91 \mathrm{mmol}, 1.0$ equiv) was mixed with $10 \mathrm{~mL} \mathrm{CH}_{2} \mathrm{Cl}_{2}$ then added via an addition funnel slowly with vigorous stirring. After addition, the reaction flask was disconnected from the nitrogen manifold and connected to air through a Drierite tube. The mixture was then stirred at rt overnight. The contents were transferred into a separatory funnel and partitioned between $10 \% \mathrm{Na}_{2} \mathrm{CO}_{3}$ and $\mathrm{CH}_{2} \mathrm{Cl}_{2}$. The organic phase was dried over anhydrous $\mathrm{Na}_{2} \mathrm{SO}_{4}$, filtered and concentrated to dryness under reduced pressure. The crude product was purified by flash column chromatography $\left(\mathrm{SiO}_{2}\right.$, 
hexanes/EtOAc/Et $3 \mathrm{~N}, 1: 5: 0.2)$ giving 6 as a colorless oil (2.65 g, $4.29 \mathrm{mmol}, 87 \%)$ : $R_{\mathrm{f}}=0.3$ (hexanes/EtOAc/Et $\left.3 \mathrm{~N}, 1: 5: 0.6\right) ;{ }^{1} \mathrm{H} \mathrm{NMR}\left(400 \mathrm{MHz}, \mathrm{CDCl}_{3}\right) \delta 7.25-7.08(\mathrm{~m}$, 9H), 6.80-6.72 (m, 4H) 6.34 (br s, 1H), 6.12 (br s, 1H), $5.64(\mathrm{~s}, 1 \mathrm{H}), 5.27(\mathrm{~s}, 1 \mathrm{H})$, $3.88(\mathrm{t}, J=6.4 \mathrm{~Hz}, 2 \mathrm{H}), 3.74(\mathrm{~s}, 3 \mathrm{H}), 3.56-3.35(\mathrm{~m}, 12 \mathrm{H}), 2.14(\mathrm{t}, J=6.4 \mathrm{~Hz}, 2 \mathrm{H})$, $1.90(\mathrm{~s}, 1 \mathrm{H}), 1.76-1.58(\mathrm{~m}, 4 \mathrm{H}), 1.50-1.40(\mathrm{~m}, 2 \mathrm{H}) ;{ }^{13} \mathrm{C} \mathrm{NMR}\left(100 \mathrm{MHz}, \mathrm{CDCl}_{3}\right) \delta$ 173.2, 168.7, 158.7, 158.2, $147.7140 .1,139.8,139.7,129.4,128.0,127.9,127.1$, $119.8,113.8,113.3,81.5,70.4,70.3,70.1,69.9,67.8,55.3,39.5,39.3,36.7,29.2$, 25.9, 25.6, 18.8; HRMS (ESI, $\left.[\mathrm{M}+\mathrm{Na}]^{+}\right) \mathrm{m} / z$ calcd for $\mathrm{C}_{36} \mathrm{H}_{46} \mathrm{~N}_{2} \mathrm{NaO}_{7}$ 641.3203, found 641.3209 .

\section{Compound 4.7}

Compound 4.6 ( $2.0 \mathrm{~g}, 3.23 \mathrm{mmol}, 1.0$ equiv) in a round-bottomed flask was stored in a dessicator over Drierite under vacuum overnight. Thymidine $(782.8 \mathrm{mg}$, $3.23 \mathrm{mmol}, 1.0$ equiv) in a round-bottomed flask was dried in the same way. The flasks were connected to a Schlenk manifold under nitrogen and the contents were further dried through multiple cycles of evacuation and nitrogen filling. To the flask containing compound 4.6, acetyl chloride (4 mL) was added, and the mixture was stirred at $\mathrm{rt}$ for $2 \mathrm{~h}$. Most of acetyl chloride was evaporated by a nitrogen flow or under vacuum. Freshly distilled hexane from the Innovative Technology's PureSolv ${ }^{\mathrm{TM}}$ system was added to precipitate the product. The hexane was removed by syringe or cannula. The residue was washed with hexane one more time, and then dried under vacuum for $1 \mathrm{~h}$. Freshly distilled pyridine $10 \mathrm{~mL}$ was added, and the solution was transferred via a cannula to the flask containing thymidine. The reaction 
mixture was stirred at rt overnight. Volatiles were evaporated under reduced pressure. The residue was transferred into a separatory funnel and partitioned between ethyl acetate and $10 \% \mathrm{Na}_{2} \mathrm{CO}_{3}$. The organic phase was dried over anhydrous $\mathrm{Na}_{2} \mathrm{SO}_{4}$ and filtered. Volatiles were removed under reduced pressure. The product was purified with flash column chromatography $\left(\mathrm{SiO}_{2}, \mathrm{EtOAc} / \mathrm{MeOH} / \mathrm{Et}_{3} \mathrm{~N}, 9.5: 0.5: 0.2\right)$ giving 4.7 as a white foam $(2.1 \mathrm{~g}, 2.50 \mathrm{mmol}, 77 \%): R_{\mathrm{f}}=0.4\left(\mathrm{EtOAc}_{\mathrm{MeOH}} / \mathrm{Et}_{3} \mathrm{~N}, 9: 1: 0.1\right) ;{ }^{1} \mathrm{H}$ NMR (400 MHz, acetone- $\left.d_{6}\right) \delta 7.62(\mathrm{~s}, 1 \mathrm{H}), 7.49-7.46(\mathrm{~m}, 2 \mathrm{H}), 7.36-7.20(\mathrm{~m}, 6 \mathrm{H})$, 6.89-6.86 (m, 5H), $6.36(\mathrm{t}, J=6.0 \mathrm{~Hz}, 1 \mathrm{H}), 5.69(\mathrm{~s}, 1 \mathrm{H}), 5.29(\mathrm{~s}, 1 \mathrm{H}), 4.59$ (br s, 1H), 4.06-4.01 (m, 2H), $3.96(\mathrm{t}, J=6.0 \mathrm{~Hz}, 2 \mathrm{H}), 3.76(\mathrm{~s}, 3 \mathrm{H}), 3.56-3.30(\mathrm{~m}, 16 \mathrm{H}), 2.38-$ $2.28(\mathrm{~m}, 2 \mathrm{H}), 2.18(\mathrm{t}, J=7.2 \mathrm{~Hz}, 2 \mathrm{H}), 1.95(\mathrm{~s}, 3 \mathrm{H}), 1.89(\mathrm{~s}, 1 \mathrm{H}), 1.45$ (s, 3H), 1.19$1.36(\mathrm{~m}, 2 \mathrm{H}) ;{ }^{13} \mathrm{C}$ NMR (100 MHz, Acetone-d 6$) \delta 172.6,168.0,163.7,159.0,158.7$, $158.5,150.6,145.2,140.8,135.9,130.3,128.3,128.0,127.0,118.6,113.8,113.3$, $110.2,86.7,86.5,84.6,71.8,70.3,70.2,69.9,69.5,67.6,64.1,59.9,54.9,40.5,39.4$, 39.1, 35.9, 25.7, 25.5, 20.1, 18.2, 13.8; HRMS (ESI, $\left.[\mathrm{M}+\mathrm{H}]^{+}\right) \mathrm{m} / \mathrm{z}$ calcd for $\mathrm{C}_{47} \mathrm{H}_{59} \mathrm{~N}_{4} \mathrm{NaO}_{11}$ 865.4000, found 865.3998.

\section{Phosphoramidite 4.1}

An oven-dried round-bottomed flask containing 4.7 (0.7 g, $0.83 \mathrm{mmol}, 1.0$ equiv) was evacuated then refilled with nitrogen. The evacuation and nitrogen-filling cycle was repeated for three times. Freshly distilled $\mathrm{CH}_{2} \mathrm{Cl}_{2}(3 \mathrm{~mL})$ was added via a syringe. 2-Cyanoethyl- $N, N, N^{\prime}, N^{\prime}$-tetraisopropylphosphoramidite $(292.2 \mu \mathrm{L}, 0.92$ mmol, 1.1 equiv) and diisopropyl ammonium tetrazolide (142.2 mg, $0.83 \mathrm{mmol}, 1.0$ equiv) were added sequentially. After stirring at $\mathrm{rt}$ for $5 \mathrm{~h}$ the mixture was 
concentrated to dryness with a nitrogen flow. The residue was purified by flash column chromatography $\left(\mathrm{SiO}_{2}, \mathrm{EtOAc} / \mathrm{Et}_{3} \mathrm{~N}, 9.5: 0.5\right)$ to give $\mathbf{6}$ as a white foam $(0.75$ g, $0.72 \mathrm{mmol}, 87 \%): R_{\mathrm{f}}=0.5($ EtOAc/acetone/Et $3 \mathrm{~N}, 7: 3: 0.1) ;{ }^{1} \mathrm{H}$ NMR $\quad(400 \mathrm{MHz}$, acetone- $\left.d_{6}\right) \delta 7.62(\mathrm{~m}, 1 \mathrm{H}), 7.50-7.47(\mathrm{~m}, 2 \mathrm{H}), 7.38-7.10(\mathrm{~m}, 6 \mathrm{H}), 6.91-6.87(\mathrm{~m}, 5 \mathrm{H})$, $6.36(\mathrm{~m}, 1 \mathrm{H}), 5.69(\mathrm{~s}, 1 \mathrm{H}), 5.29(\mathrm{~s}, 1 \mathrm{H}), 4.76(\mathrm{br} \mathrm{s}, 1 \mathrm{H}), 3.98-3.80(\mathrm{~m}, 2 \mathrm{H}), 3.78(\mathrm{~s}$, $6 \mathrm{H}), 3.6-3.20(\mathrm{~m}, 8 \mathrm{H}), 2.75(\mathrm{t}, J=6.0 \mathrm{~Hz} 2 \mathrm{H}), 2.61(\mathrm{t}, J=6.4 \mathrm{~Hz}, 2 \mathrm{H}) 2.53-2.44(\mathrm{~m}$, 2H), $2.18(\mathrm{t}, J=7.2 \mathrm{~Hz}, 2 \mathrm{H}), 1.90(\mathrm{~s}, 3 \mathrm{H}), 1.86-1.60(\mathrm{~m}, 4 \mathrm{H}), 1.48-1.47(\mathrm{~m}, 6 \mathrm{H})$, 1.27-1.14 (m, 12H); ${ }^{13} \mathrm{C}$ NMR (100 MHz, Acetone- $\left.\mathrm{d}_{6}\right) \delta$ 172.4, 167.9, 163.7, 159.1, $158.6,150.6,145.1,140.8,130.4,128.4,128.0,127.1,118.5,113.9,113.4,110.5$, $94.6,86.8,85.5,84.6,73.8,70.4,70.2,69.9,69.6,67.8,63.6,58.9,54.9,43.3,43.2$, $39.4,39.1,35.9,25.8,25.5,24.3,20.0,18.3,11.6 ;{ }^{31} \mathrm{P}$ NMR (160 MHz, acetone- $\left.d_{6}\right) \delta$ 149.3, 149.2, LRMS (ESI, $[\mathrm{M}+\mathrm{Na}]^{+}$) $\mathrm{m} / z$ calcd for $\mathrm{C}_{55} \mathrm{H}_{75} \mathrm{~N}_{6} \mathrm{NaO}_{12} \mathrm{P} 1065.5$, found 1065.4

\section{Procedure for Small Scale ODN Purification}

\section{ODN Synthesis}

The 20-mer ODN 4.8 was used as an example. Its synthesis was carried out on a $1 \mu \mathrm{mol}$ scale under standard conditions using phosphoramidite chemistry. The synthesizer manufacturer suggested $1 \mu \mathrm{mol}$ synthetic cycle was copied to a new file. After the coupling steps and before the capping steps, a 180 second waiting step was inserted, and was set to be active only for base 5. The $0.1 \mathrm{M}$ solution of 4.1 in dry acetonitrile was attached to bottle position 5. The volume should be around $600 \mu \mathrm{L}$, which was needed for running the bottle exchange cycle, the begin cycle, as well as 
the coupling steps in one cycle. When editing sequence, the dT at the 5'-end was input as 5. CPG with a long chain alkylamine succinyl ester linker and a glass pore size of $500 \AA$ was used as solid support. The 5'-DMTr 2-cyanoethyl benzoyl-dA, isobutyryl-dG, acetyl-dC, and dT phosphoramidites ( $0.1 \mathrm{M}$ acetonitrile solutions) were used as coupling monomers. The $0.25 \mathrm{M}$ DCI solution in acetonitrile was used as activator. Failure sequences were capped with $\mathrm{Ac}_{2} \mathrm{O}$ in each synthetic cycle. At the end of synthesis, detritylation was not carried out as this would remove the methacrylamide tag. Cleavage was carried out on the synthesizer with concentrated $\mathrm{NH}_{4} \mathrm{OH}$. Deprotection was achieved by heating the solution to $55^{\circ} \mathrm{C}$ for $25 \mathrm{~h}$. After cooling to $\mathrm{rt}, 200 \mu \mathrm{L}$ DIEA was added. The solution was divided into 5 equal portions, and evaporated to dryness under vacuum. One portion $(\sim 0.2 \mu \mathrm{mol})$ was dissolved in $300 \mu \mathrm{L}$ water, $20 \mu \mathrm{L}$ was injected into RP HPLC to generate trace a (Figure 4.2).

\section{Polymerization}

To the second portion $(\sim 0.2 \mu \mathrm{mol})$ of ODN in a centrifuge tube was added 50 $\mu \mathrm{L}$ water. The tube was vortexed and spun shortly for efficient dissolution. Polymerization solution $\left[12 \mu \mathrm{L}, 44.4 \mu \mathrm{mol} N, N\right.$-dimethylacrylamide, $4.4 \mu \mathrm{mol} N, N^{\prime}$ methylenebis(acrylamide)], and initiators $\left(\mathrm{NH}_{4}\right)_{2} \mathrm{~S}_{2} \mathrm{O}_{8}(5 \%, 5 \mu \mathrm{L}, 1.1 \mu \mathrm{mol})$ and TMEDA $(0.66 \mathrm{M}, 5 \mu \mathrm{L}, 3.3 \mu \mathrm{mol})$ were added sequentially. The mixture was vortexed and spun briefly, and then transferred immediately into the top compartment of a centrifugal filter unit. The bottom of the compartment could be stopped with a cap or parafilm, but the solution will not pass through the filter disc even without 
stopping. Care needs to be taken to transfer all solution to the center of the filter disc, which can be achieved with steady releasing and pushing the pipette. Washing the residue solution to the compartment is not recommended. The solution was allowed to stand at rt with the cap of the unit closed. A gel was formed in $~ 15$ min, but the polymerization was allowed to continue for $1 \mathrm{~h}$.

\section{Washing}

The gel was loosened with a clean spatula, and water containing $5 \% \mathrm{Et}_{3} \mathrm{~N}$ $(\sim 200 \mu \mathrm{L})$ was added. After $3 \mathrm{~min}$, the liquid was removed with a spin. The washing was repeated for 7 times with the last wash using pure water.

\section{Cleavage and Extraction}

A minimum amount of $\mathrm{AcOH}$ solution (80\%) that could cover the gel ( 120 $\mu \mathrm{L}$ ) was added. After $5 \mathrm{~min}$, the liquid was collected with a spin. The procedure was repeated for two more times. A minimum amount of water that could cover the gel $(\sim 120 \mu \mathrm{L})$ was added, and the extract was collected with a spin. The extraction was repeated for four times. The extracts including those of $\mathrm{AcOH}$ solution and water were combined and evaporated to dryness. To the residue, concentrated $\mathrm{NH}_{4} \mathrm{OH}$ (100 $\mu \mathrm{L}$ ) was added. After a short vortex, the closed tube was heated at $80{ }^{\circ} \mathrm{C}$ for $15 \mathrm{~min}$. The tube was cooled to rt, and $n \mathrm{BuOH}(900 \mu \mathrm{L})$ was added. The mixture was vortexed for $30 \mathrm{sec}$ and centrifuged for $3 \mathrm{~min}$ at $14.1 \mathrm{~K}$. The supernatant was carefully removed with a pipette, and the residue, which was the pure ODN, was dried under vacuum to evaporate residue $n \mathrm{BuOH}$. The ODN was dissolved in $300 \mu \mathrm{L}$ water, 
vortexed, and spun, and $20 \mu \mathrm{L}$ was injected into RP HPLC to generate traces b (Figure 2). The recovery yield of the ODN was determined to be $68 \%$ by dividing the area of the peak in trace $b$ at 19 minutes by that in trace a at 45 minutes.

The 31-mer ODN 4.9 was synthesized under the same conditions as describe for 4.8 except that cleavage and deprotection were achieved using a mixture of concentrated $\mathrm{NH}_{4} \mathrm{OH}$ and $\mathrm{MeNH}_{2}$ solutions $(1: 1 \mathrm{v} / \mathrm{v})$ at $\mathrm{rt}$ for $3 \mathrm{~h}$. The ODN was purified at $0.2 \mu \mathrm{mol}$ scale using the same procedure and the same amount of reagents for purifying 4.8. The RP HPLC traces for crude and pure ODNs are in supporting information. The recovery yield of purification was determined to be $71 \%$ by comparing areas of peaks in the HPLC profiles of pure and crude ODN. The 37-mer ODN 4.10 was also purified at $1 \mu \mathrm{mol}$ scale using the same procedure with slightly different amount of reagents: $50 \mu \mathrm{L}$ water for dissolving crude ODN, $24 \mu \mathrm{L}$ polymerization solution for catching full-length sequences, $5 \mu \mathrm{L}\left(\mathrm{NH}_{4}\right)_{2} \mathrm{~S}_{2} \mathrm{O}_{4}$ and TMEDA solutions for initiating polymerization, and minimum amount of solutions that could cover gel for washing failure sequences and for cleavage and extraction. RP HPLC profiles for crude and pure ODN are in supporting information. Recovery yield was determined to be $83 \%$ by comparing areas of peaks in the HPLC profiles of pure and crude ODN.

\section{Procedure for Large Scale Purification}

The 37-mer ODN 4.10 was used as an example. Its synthesis was achieved in five $10 \mu \mathrm{mol}$ columns with $1000 \AA \mathrm{CPG}$. The same procedure and reagents for synthesizing 4.8 were used except that a different synthetic cycle was used. The 
standard $10 \mu \mathrm{mol}$ synthesis cycle was copied into a new file. After the coupling steps and before the capping steps, a 180-second waiting step was inserted, and was set to be active only for base 5 . Approximately, a total of $5.9 \mathrm{~mL}$ of $0.08 \mathrm{M}$ solution of 4.1 was used for tagging full-length sequences. According to trityl essay before the cycle for attaching 4.1, the average yield of 36-mer for the five synthesis was 50\%. Cleavage and deprotection were achieved under the same conditions for 4.9. DIEA $(\sim 500 \mu \mathrm{L})$ was added to minimize the possibility of premature tag cleavage during evaporation. RP HPLC analysis of the crude indicated that only about $50 \%$ of the $36-$ mer intermediate was successfully methacrylated with 4.1 (trace c, Figure 2). As a result, the synthesis yield of the 37 -mer was $50 \%$. For purification, the crude ODN was dissolved in $1.25 \mathrm{~mL}$ water in a $25 \mathrm{~mL}$ round-bottomed flask, which was flushed with nitrogen. Polymerization solution $(1.25 \mathrm{~mL})$ and initiator solutions (10 $\mu \mathrm{L}$ each) were added sequentially. The flask was hand-shaken briefly, and polymerization was allowed to proceed at $\mathrm{rt}$ for $1 \mathrm{~h}$. The gel was transferred into a $15 \mathrm{~mL}$ Büchner funnel with a sintered glass disc and broken into several pieces with a spatula. Water ( 4 $\mathrm{mL}$ ) containing 5\% $\mathrm{Et}_{3} \mathrm{~N}$ was added to the gel. After 3 min, vacuum was applied and the liquid was removed. The washing was repeated for 4 times. The gel was further washed with water $(\sim 4 \mathrm{~mL})$ containing $1 \% \mathrm{Et}_{3} \mathrm{~N}$ for 4 times without waiting between the washes, and finally, the gel was washed with pure water $(\sim 4 \mathrm{~mL})$. For cleaving full-length sequences, $80 \% \mathrm{AcOH}(\sim 4 \mathrm{~mL})$ was added into the funnel. After $5 \mathrm{~min}$, vacuum was applied and the extract was collected in a centrifuge tube placed in the filter flask. The procedure was repeated for two times. The gel was further washed with water $(4 \mathrm{~mL} \times 5)$ without waiting between washes. The extracts including $\mathrm{AcOH}$ 
solution and water were combined and evaporated to dryness under vacuum. The residue was dissolved in $1 \mathrm{~mL}$ concentrated $\mathrm{NH}_{4} \mathrm{OH}$ and heated at $80{ }^{\circ} \mathrm{C}$ for $15 \mathrm{~min}$. After cooling to rt, $9 \mathrm{~mL} n \mathrm{BuOH}$ was added, and the mixture was vortexed for $30 \mathrm{sec}$ and centrifuged for 3 min. The supernatant was removed, and the residue was dried under vacuum. Pure ODN was obtained as white cotton-like fluffy fibers (see picture in supporting information). The weight was $98 \mathrm{mg}$, which corresponds to a $70 \%$ recovery yield of the purification procedure. A portion of the ODN was dissolved in water, and analyzed with RP HPLC (trace d, Figure 2), which indicated 100\% purity. The images of ${ }^{1} \mathrm{H}$ NMR of the pure ODN are in supporting information.

\section{Procedure for Long Sequence Purification}

The sequences 4.11 (61-mer) and 4.12 (151-mer) were used to demonstrate the power of the technique for purification of long ODN sequences. ODN 4.12 is used for the description of the procedure. The ODN was synthesized at $0.2 \mu$ mol scale under similar conditions described for $\mathbf{8}$ with slight modifications. The standard 0.2 $\mu \mathrm{mol}$ (instead of $1 \mu \mathrm{mol}$ ) synthetic cycle was used to create the new cycle. After coupling and capping steps, additional waiting steps of $45 \mathrm{sec}$ and $30 \mathrm{sec}$ were added, respectively. CPG with a pore size of $2000 \AA$ was used as solid support. Before synthesis, the capping solutions were manually delivered to fill the column, and the mixture was allowed to stand at $\mathrm{rt}$ for $20 \mathrm{~min}$. The CPG was then washed with acetonitrile. All other conditions were the same as for 4.8. The synthesis yield before the last cycle was $22 \%$ according to trityl essay. The ODN was cleaved and deprotected using the mixture of concentrated $\mathrm{NH}_{4} \mathrm{OH}$ and $\mathrm{MeNH}_{2}(1: 1 \mathrm{v} / \mathrm{v})$ at $\mathrm{rt}$ for 
$3 \mathrm{~h}$. About $200 \mu \mathrm{L}$ DIEA was added, and the solution was divided into five equal portions, which were evaporated to dryness under vacuum. One portion was dissolved in $150 \mu \mathrm{L}$ water, and $20 \mu \mathrm{L}$ was injected into RP HPC to generate trace e (Figure 4.2). A second portion was used for purification. The polymerization step including reagent volumes were exactly the same as for 4.8. In the washing step, 3.0 M NaOAc (200 $\mu \mathrm{L} \times 6$ with 3 min waiting between the washes), water containing $1 \% \mathrm{Et}_{3} \mathrm{~N}$ (200 $\mu \mathrm{L} \times 1)$ and pure water $(200 \mu \mathrm{L} \times 1)$ were used, sequentially. Cleavage, extraction, and precipitation were carried out under the same conditions for 4.8. The pure ODN was dissolved in $150 \mu \mathrm{L}$ water, and $20 \mu \mathrm{L}$ was injected into RP HPLC (flow rate 2 $\mathrm{mL} / \mathrm{min}$, column temperature $55{ }^{\circ} \mathrm{C}$ ) to give trace $\mathrm{f}$. The recovery yield of the purification process was determined to be $50 \%$ by comparing the area of peaks of crude trace $(37 \mathrm{~min})$ with that of pure one $(18 \mathrm{~min})$.

The sequence 4.11 was synthesized at $1 \mu \mathrm{mol}$ scale under the conditions for 4.9 using $1000 \AA$ APG. Purification was carried out at $0.2 \mu \mathrm{mol}$ scale under the same conditions described for 4.9. The recovery yield was determined to be $83 \%$ by comparing the area of peaks of crude trace $(40 \mathrm{~min})$ with that of pure one $(20 \mathrm{~min})$. HPLC images of $\mathbf{1 1}$ and $\mathbf{1 3}$ are in supporting information.

\subsection{Conclusion}

A new methacrylation phosphoramidite that contains a linker cleavable with acetic acid solution has been synthesized, and used for the purification of ODN using the catching full-length sequences by polymerization approach. The simplicity, convenience, and suitability of the technology with the new linker for small scale 
purification, large scale purification and long sequence purification have been demonstrated. Because acetic acid solution is much more environmentally benign than the previously used hydrogen fluoride for the same purpose, we believe that the work described in this paper will promote wide adoptions of the ODN purification technology in academic labs, biotechnology companies and pharmaceutical industry.

\section{Acknowledgements}

Financial support from US NSF (CHE-0647129 and CHE-1111192), Michigan Translational Research \& Commercialization (M-TRAC) of Michigan Economic Development Corporation, Michigan Tech Research Excellence Fund for Technology Commercialization (REF-TC), and Michigan Universities Commercialization Initiatives (MUCI); assistance from Mr. Dean W. Seppala (electronics) and Mr. Jerry L. Lutz (NMR); and NSF equipment grants (CHE1048655 and CHE-9512455) are gratefully acknowledged. 


\section{References and Notes}

1. Singh, Y., P. Murat, and E. Defrancq, Recent developments in oligonucleotide conjugation. Chemical Society Reviews, 2010, 39(6): p. 2054-2070.

2. Juliano, R.L., X. Ming, and O. Nakagawa, The Chemistry and Biology of Oligonucleotide Conjugates. Accounts of Chemical Research, 2012, 45(7): p. 1067-1076.

3. Fiszer, A. and W.J. Krzyzosiak, Oligonucleotide-based strategies to combat polyglutamine diseases. Nucleic Acids Research, 2014, 42(11): p. 6787-6810.

4. Grijalvo, S., A. Avino, and R. Eritja, Oligonucleotide delivery: a patent review (2010-2013). Expert Opinion on Therapeutic Patents, 2014, 24(7): p. 801-819.

5. Castanotto, D. and C.A. Stein, Antisense oligonucleotides in cancer. Current Opinion in Oncology, 2014, 26(6): p. 584-589.

6. Borovkov, A.Y., A.V. Loskutov, M.D. Robida, et al., High-quality gene assembly directly from unpurified mixtures of microarray-synthesized oligonucleotides. Nucleic Acids Research, 2010, 38(19): p. 1-10.

7. Helwa, Y., N. Dave, R. Froidevaux, et al., Aptamer-Functionalized Hydrogel Microparticles for Fast Visual Detection of Mercury(II) and Adenosine. Acs Applied Materials \& Interfaces, 2012, 4(4): p. 2228-2233.

8. Mueller, S., J.R. Coleman, and E. Wimmer, Putting Synthesis into Biology: A Viral View of Genetic Engineering through De Novo Gene and Genome Synthesis. Chemistry \& Biology, 2009, 16(3): p. 337-347. 
9. Kosuri, S. and G.M. Church, Large-scale de novo DNA synthesis: technologies and applications. Nature Methods, 2014, 11(5): p. 499-507.

10. Tian, J.D., K.S. Ma, and I. Saaem, Advancing high-throughput gene synthesis technology. Molecular Biosystems, 2009, 5(7): p. 714-722.

11. Hughes, R.A., A.E. Miklos, and A.D. Ellington, Gene Synthesis: Methods and Applications. Synthetic Biology, Pt B, 2011, 498: p. 277-309.

12. Abramova, T., Frontiers and Approaches to Chemical Synthesis of Oligodeoxyribonucleotides. Molecules, 2013, 18(1): p. 1063-1075.

13. Schulte, M., N. Luhring, A. Keil, et al., Purification of DMT-on oligonucleotide by simulated moving-bed (SMB) chromatography. Organic Process Research \& Development, 2005, 9(2): p. 212-215.

14. Sanghvi, Y.S. and M. Schulte, Therapeutic oligonucleotides: The state-of-theart in purification technologies. Current Opinion in Drug Discovery \& Development, 2004, 7(6): p. 765-776.

15. Deshmukh, R.R., W.E. Leitch, and D.L. Cole, Application of sample displacement techniques to the purification of synthetic oligonucleotides and nucleic acids: a mini-review with experimental results. Journal of Chromatography A, 1998, 806(1): p. 77-92.

16. Deshmukh, R.R., T.N. Warner, F. Hutchison, et al., Large-scale purification of antisense oligonucleotides by high-performance membrane adsorber chromatography. Journal of Chromatography A, 2000, 890(1): p. 179-192. 
17. Fang, S., S. Fueangfung, X. Lin, et al., Synthetic oligodeoxynucleotide purification by polymerization of failure sequences. Chemical Communications, 2011, 47(4): p. 1345-1347.

18. Fang, S.Y. and S. Fueangfung, Scalable Synthetic Oligodeoxynucleotide Purification with Use of a Catching by Polymerization, Washing, and Releasing Approach. Organic Letters, 2010, 12(16): p. 3720-3723.

19. Pokharel, D., Y. Yuan, S. Fueangfung, et al., Synthetic Oligodeoxynucleotide Purification by Capping Failure Sequences with a Methacrylamide Phosphoramidite Followed by Polymerization. RSC Advances, 2014, 4: p. 8746-8757.

20. Pokharel, D. and S. Fang, A Highly Convenient Procedure for Oligodeoxynucleotide Purification. The Open Organic Chemistry Journal, 2014, 8: p. 15-18.

21. Yuan, Y.N., S. Fueangfung, X. Lin, et al., Synthetic 5'-phosphorylated oligodeoxynucleotide purification through catching full-length sequences by polymerization. RSC Advances, 2012, 2(7): p. 2803-2808.

22. Tang, W. and S.Y. Fang, Mono-acylation of symmetric diamines in the presence of water. Tetrahedron Letters, 2008, 49(41): p. 6003-6006.

23. Pappas, K., X. Zhang, W. Tang, et al., Phenyl esters, preferred reagents for mono-acylation of polyamines in the presence of water. Tetrahedron Letters, 2009, 50(41): p. 5741-5743.

24. Pearson, W.H., D.A. Berry, P. Stoy, et al., Fluorous affinity purification of oligonucleotides. Journal of Organic Chemistry, 2005, 70(18): p. 7114-7122. 


\section{Chapter 5 \\ Introduction to Peptide Purification}

\subsection{Peptides}

Peptides are short polymers of amino acids monomers. The amino acids are linked together by amide bonds from a dehydration reaction between a carboxylic group of one amino acid and amino group of another amino acid. A general structure of peptides is shown in Figure 5.1 (5.1). Depending on the number of amino acid monomers, peptides are classified as dipeptides, tripeptides, tetrapeptides and so on. Peptides with 10 or less number of amino acid are known as oligopeptides while the longer peptides (more than 100 amino acids) are called proteins. The carboxylic end of a peptide is termed as its $\mathrm{C}$-terminal and amino end is $\mathrm{N}$-terminal.

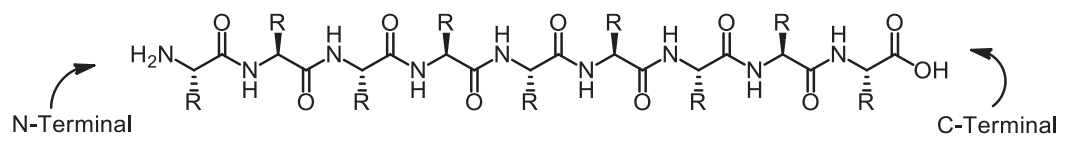

5.1

Figure 5.1. Peptide structure

\subsection{Applications of Peptides}

Synthetic peptides have a wide range of application in therapeutics, biochemistry, molecular biology and immunology.[1] One of the most important applications of peptides is to use as medicine. There are about 60 peptide drugs on the market and more than 500 are in different stages of clinical and pre-clinical trials. It is estimated the global annual sale of peptide drugs in 2010 to be $\$ 13$ billion [2]. Peptide drugs have several advantages over traditional small molecule drugs. They 
usually have high affinity, and selectivity to biological targets, and therefore have high potency and low toxicity. Another reason for low toxicity of peptide drugs is because the degradation products of peptide drug are amino acids, which are known non-toxic molecules in the nature.[3] Currently, peptide based drugs are used to treat different types of cancer, cardiovascular diseases and other diseases. They are also used as vaccines for immunization against hepatitis $\mathrm{A}$ and $\mathrm{B}$, insulin for diabetes and some growth hormones.[4] It is estimated that the annual sale of peptide drugs is 20 billion USD in 2012, which is about $2 \%$ of total drug product. Currently, the annual growth rate of peptide based drug sale is $7.5-10 \%$.[5]

\subsection{Peptide Synthesis}

Peptides are synthesized by coupling of the carboxylic group of one amino acid with the amino group of another amino acid. Two types of methods are commonly used. One is solution-phase synthesis. This method is classical. It needs intensive labor to purify intermediate products. It is still widely used. However, the most commonly used method, which is the second method, is solid-phase synthesis. This method was first developed by Bruce Merrifield in 1963.[6] Using this method, the first amino acid is anchored to a solid support, usually polystyrene beads. Peptides are assembled by stepwise addition of amino-protected amino acid monomers. Because the desired peptide intermediates are anchored to the solid support, after each reaction during the multiple step synthesis, excess reagents and side products are conveniently removed by simple washing. As a result solid-phase synthesis is rapid and can be automated. Nowadays, solid support with first amino acid being attached 
is commercially available. Scheme 5.1 illustrates the typical solid phase peptide synthesis cycle that are responsible for attaching one amino acid to the nascent peptides on solid support. The cycle consists of three steps which are deprotection, coupling and capping. They are described in following paragraphs.

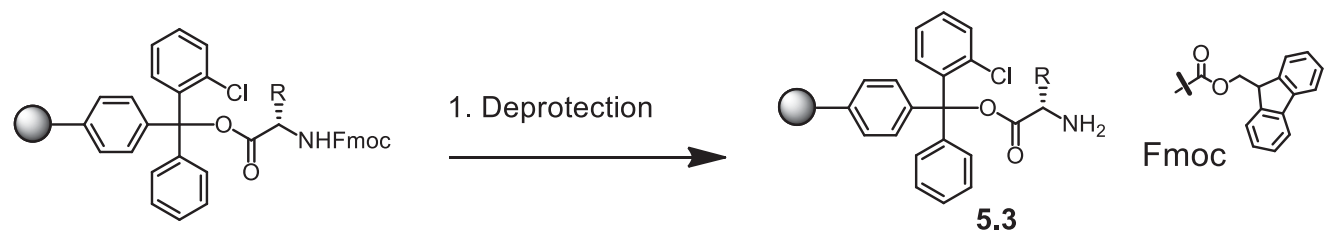

5.2
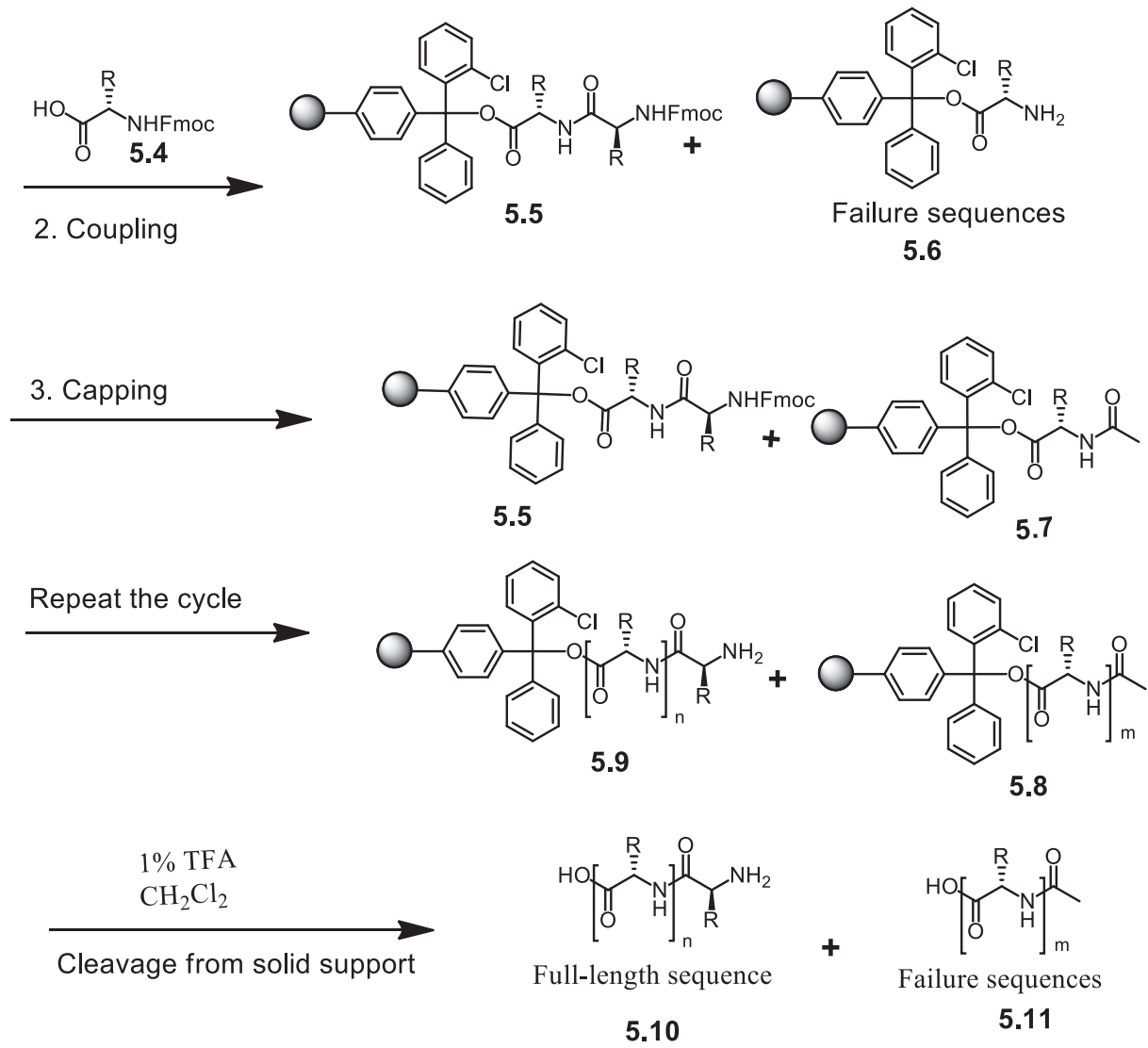

Scheme 5.1. Synthesis cycle of solid-phase peptide synthesis.

\subsubsection{Side-Chain Functional Group Protection}


Some amino acids contain a side chain functional group. They have to be protected during the peptide synthesis. These protecting groups should remain intact throughout the synthesis and must be readily removable after synthesis. Most commonly used side chain protecting groups are tert-butyloxycarbonyl (Boc), trityl, benzoyloxymethyl and tert-butylether. Nowadays, side-chain protected amino acids are commercially available.

\subsubsection{Deprotection}

This is the first step of a peptide synthesis cycle. As shown in scheme 5.1, in this step, 5.2, which is the first amino acid anchored to solid support with its amino group being protected with a Fmoc group, is converted to 5.3. The Fmoc group is an abbreviation of 9-fluorenylmethoxycarbonyl group. This step is usually achieved with the mild base piperidine, usually a $20 \%$ solution in dimethyl formamide (DMF). The acid-labile protecting group used for side chain protection are all stable under the conditions. Fmoc is the most widely used amino protecting group. Besides the Fmoc group, the Boc group which is an abbreviation of tert-butyloxycarbonyl is also widely used. Boc group is removed under acidic conditions. The side chain protecting groups are also removed under acidic conditions but this needs harsher conditions. After this step, the amino group in $\mathbf{5 . 3}$ is free of protecting group and the solid support is now ready for the next step, which is coupling. 


\subsubsection{Coupling}

The second step in a peptide synthesis cycle is coupling. As shown in scheme 5.1, this step couples $\mathbf{5 . 3}$ with the Fmoc-protected amino acid monomer $\mathbf{5 . 4}$ to give 5.5. This step requires activation of carboxylic group of the amino acid 5.4. Most commonly used coupling reagents include $N, N$ '-dicyclohexylcarbodiimide (DCC) 5.12 and diisopropylcarbodiimide (DIC) 5.13. Recently, $O$-(7-azabenzotriazol-1-yl)$N, N, N^{\prime}, N^{\prime}$-tetramethyluronium hexafluorophosphate (HATU) $5.14 O$-(benzotriazol-1yl)- $N, N, N^{\prime}, N^{\prime}$-tetramethyluronium hexaflurophosphate (HBTU) 5.15 are widely used because these reagents reduce racemization.[7] These coupling reagents form highly reactive intermediates with the carboxylic acid group of amino acid monomer (5.4), which react with $\mathbf{5 . 3}$ to form the peptide bond in $\mathbf{5 . 5}$.

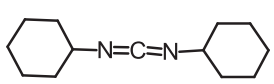

5.12

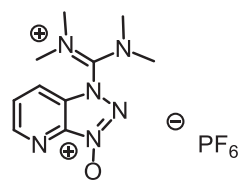

$\Theta$

5.14

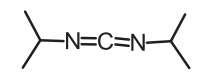

5.13

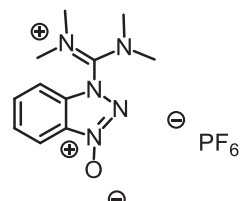

5.15

Figure 5.2. Example peptide coupling reagents 


\subsubsection{Capping}

The third step in a peptide synthesis is capping. The coupling step is usually very efficient but it may be incomplete. The unreacted amino group in $\mathbf{5 . 3}$ if not capped, can participate in the coupling reactions in the next synthesis cycle. This generates deletion sequences. Deletion sequences are very difficult to remove during purification. To solve the problem, the unreacted amino groups are capped with large excess of acetic anhydride right after each coupling step to give 5.7. Products $\mathbf{5 . 7}$ are called failure sequences. They cannot participate in next coupling reactions.

The synthesizer repeats the steps deprotection, coupling and capping, which is responsible to add one amino acid in each synthesis cycle until desired length of peptide is obtained.

\subsubsection{Cleavage and Deprotection}

Once the peptide synthesis is complete, the product is cleaved from solid support. Cleavage is usually achieved with an acid. In the example we shown in chapter 6, we used the 2-chlorotrityl linker to anchor nascent peptides to solid support. The cleaving agent is $1.0 \%$ solution of trifluoroacetic acid (TFA) in dichloromethane. Under these conditions, side chain protecting groups are not removed. To remove side chain protecting group, usually the TFA cocktail containing $81.5 \%$ TFA, $1.0 \%$ triisopropylsilane, $5.0 \%$ water, $2.5 \%$ ethane dithiol, $5.0 \%$ thioanisole and 5.0\% phenol is used. This cocktail is much more acidic and can remove all side chain protecting groups. Because the side products of the deprotection 
reactions include highly reactive cationic species, the cocktail contains scavengers, which prevents reactive cationic species from reacting with deprotected peptides.

\subsection{Impurities}

After synthesis, cleavage and deprotection, the crude peptide contains the desired full-length sequence and impurities including failure sequences. The impurities are divided into different classes. They have to be removed before most of the applications.

The first type of impurities is the failure sequences. They are generated from incomplete coupling of amino acid monomer during synthesis. Incomplete removal of Fmoc group in the deprotection step in a synthesis cycle also results in failure sequences.[8] Because the failure-sequences have similar physical properties as the full-length peptides, they are difficult to remove. The major challenge of peptide purification is to remove the failure-sequences.

The second type of impurities include deletion and addition sequences. The deletion sequences are resulted from incomplete capping. The unreacted amino group can couple with amino acid in the next synthesis cycle. The deletion sequence lacks one or more amino acids in the peptide chain. The addition sequences are resulted from premature removal of Fmoc group. The addition sequences have at least one or more amino acids. Both deletion and addition sequences are challenge to remove during purification. The best strategy is to minimize their quantity to close to zero by tuning the reaction conditions during synthesis. 
The third type of impurities is small organic molecules from side chain protecting groups. These impurities can be removed easily by precipitation with cold diethyl ether from TFA solution. The peptides are not soluble in cold ether, and are precipitated. The small organic molecules are soluble in ether, and remain in solution.

Other impurities include diasteromers which are resulted from racemization. Some impurities are generated from modification of functional groups.[9] These impurities should be minimized by tuning the condition during synthesis, cleavage and deprotection. They are challenging to remove using any purification methods.

\subsection{Current Methods of Purification}

Currently, several methods are extensively studied for peptide purification. These methods include reversed-phase (RP) HPLC, fluorous affinity purification, polyacrylamide gel-electrophoresis, size-exclusion chromatography, and ionexchange chromatography. The principles of the methods have been introduced in Chapter 1. When they are applied to peptide purification, advantages and disadvantages similar to ODN purification exist. Briefly the disadvantages include high labor demand, unamenable for automation, requiring expensive instrument and column consuming large volumes of harmful organic solvents, expensive to scale up, and harmful to environment.

\subsection{Our Peptide Purification Method}

To overcome the problems associated with known peptide purification methods, we developed a simple and convenient method for peptide purification. This 
method utilizes the same principle as discussed in previous chapters for ODN purification, which is catching by polymerization. In this method, a compound containing polymerizable methacrylamide group and a cleavable linker (5.16) is attached to the amino terminus of full-length peptide. The failure sequences are not tagged with the compound. During purification, the crude product is subjected to polymerization. The full-length sequences are incorporated into polymer. The failure sequences are removed by washing. The full-length sequences are then cleaved from the polymer. The compound we used for the application is 5.16. It contains a methacrylamide polymerizable group and an acid-cleavable linker. The following paragraph explains how to tag the full-length sequence while leaving failure sequences free of the methacrylamide tag.

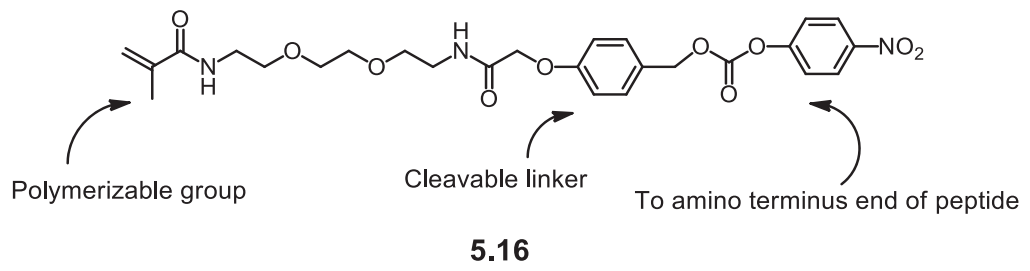

Figure 5.3. Polymerizable linker

As shown in Scheme 5.2, at the end of peptide synthesis, the full-length peptide on solid support (5.9) is reacted with the polymerizable tagging agent $\mathbf{5 . 1 6}$ to give 5.17. The failure sequences on the solid support $\mathbf{5 . 8}$ do not react because their amino groups are capped with acetic anhydride in capping step. The peptide including full-length sequences and failure sequences are cleaved from the solid support. The 
cleavage reagent we used is $1 \%$ TFA. The linker shown in $\mathbf{5 . 1 6}$ and side chain protecting groups are stable under this condition.

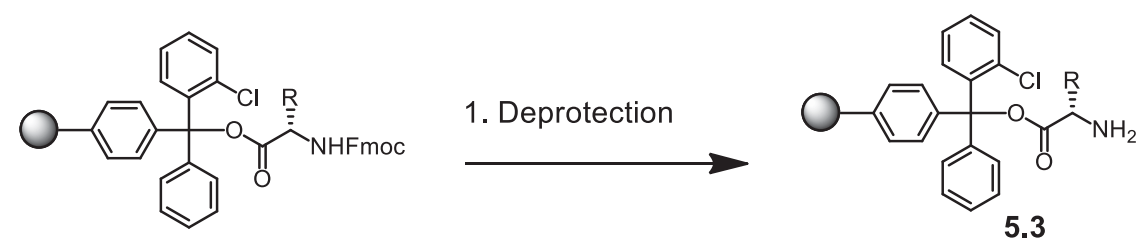

5.2

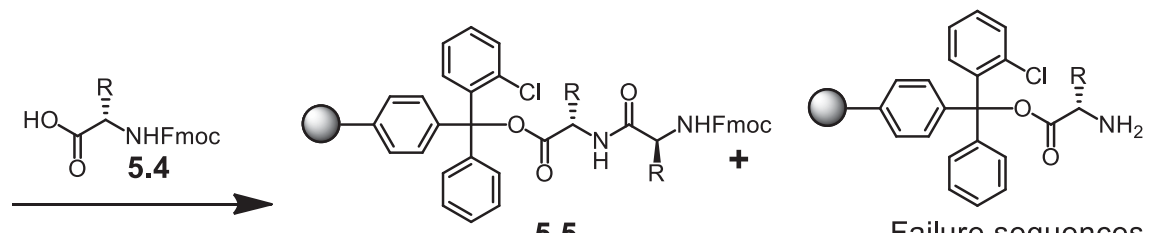

2. Coupling

Failure sequences

5.6
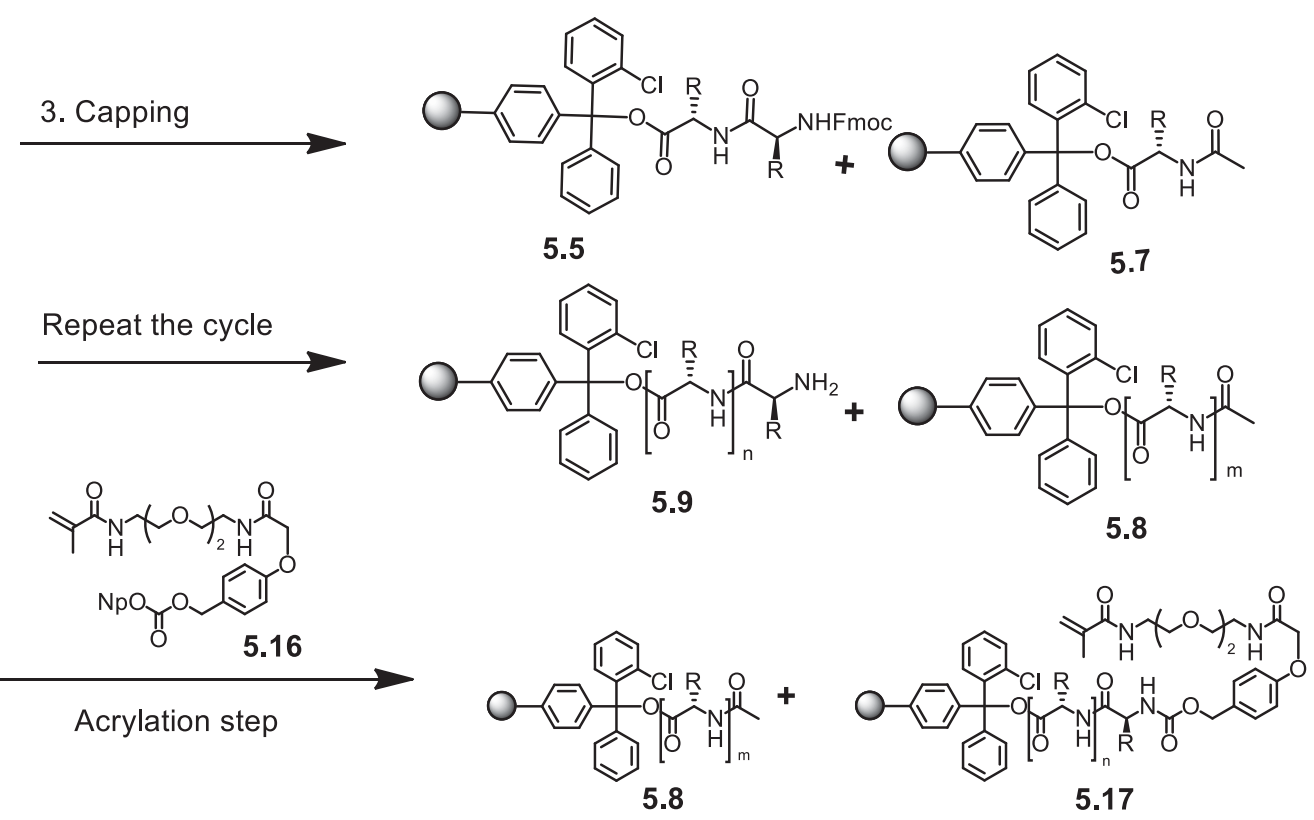

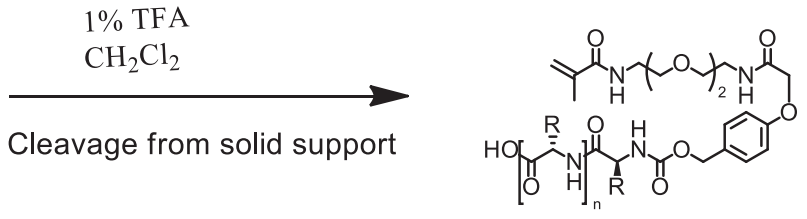

5.18

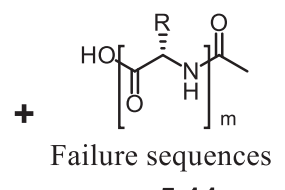

5.11

Scheme 5.2. Acrylation of full-length peptide by polymerizable linker $\mathbf{5 . 1 6}$ 
After peptide synthesis and cleavage, the crude peptide mainly contains the methacrylamide-tagged full-length sequence 5.18, the failure sequences $\mathbf{5 . 1 1}$ and other impurities. It is subjected into a polymerization mixture containing $\mathrm{N}, \mathrm{N}$ dimethylacrylamide 5.19 and $N, N$-methylenebisacrylamide 5.20. The polymerization reaction is initiated with ammonium persulfate (APS) and $N, N, N^{\prime}, N^{\prime}-$ tetramethylethylenediamine (TMEDA). The full-length peptide $\mathbf{5 . 1 8}$ is incorporated into the polymer gel 5.21. The failure sequences $\mathbf{5 . 1 1}$ and other impurities remain in solution. The failure sequences and other impurities are washed away. The full-length peptide is cleaved with TFA cocktail solution, which also removes the side chain protecting groups. The full-length peptide is further extracted with TFA. Small molecule impurities are removed by precipitation of peptides with diethyl ether from the TFA solution. The small molecules are in solution. The peptide is not soluble in ether and is precipitated.
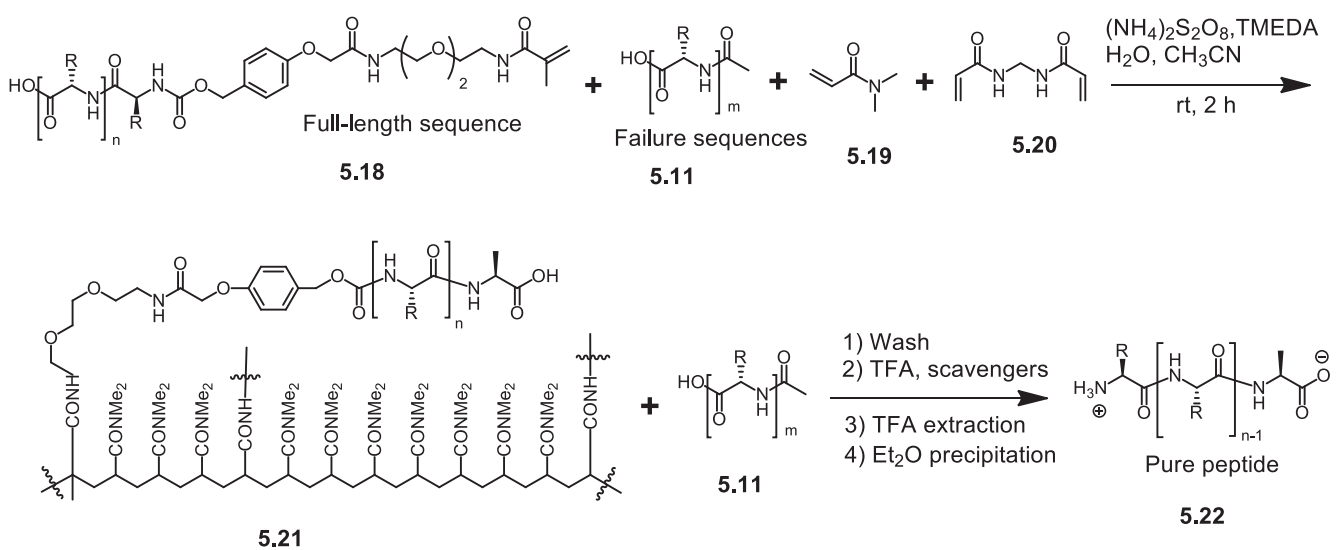

Scheme 5.3. The catching full-length sequence by polymerization peptide purification method 


\subsection{Advantages of Our New Peptide Purification Method}

Our new peptide purification method has significant advantages over all known purification methods. The method does not involve any chromatography and purification is achieved through simple manipulation such as mixing, shaking, filtration and extraction. It does not need any expensive reagents, instruments and column. Therefore it is highly suitable for large scale purification including large scale peptide based drug purification.

The advantages of our technology to purify ODN are presented in Table $\mathbf{1 . 1}$ and Table 1.2 of chapter 1. The tables can be used to compare the advatages of our technology for peptide purification.

The detailed information on peptide purification using the catching full-length sequence by polymerization method is presented in Chapter 6. 


\section{References and Notes}

1. Moser, R., S. Klauser, T. Leist, et al., Applications of Synthetic Peptides. Angewandte Chemie International Edition in English, 1985, 24(9): p. 719727.

2. Lax, R., The future of peptide development in the pharmaceutical industry. PharManufacturing: The International Peptide Review, 2010: p. 10-15.

3. Vlieghe, P., V. Lisowski, J. Martinez, et al., Synthetic therapeutic peptides: science and market. Drug Discovery Today, 2010, 15(1-2): p. 40-56.

4. Antosova, Z., M. Mackova, V. Kral, et al., Therapeutic application of peptides and proteins: parenteral forever? Trends in Biotechnology, 2009, 27(11): p. 628-635.

5. Mezo, G., Peptide and protein based pharmaceuticals. Amino Acids, Peptide and Proteins, 2013, 38: p. 203.

6. Erickson, B.W. and R. Merrifield, Solid-phase peptide synthesis. The Proteins, 1976, 2: p. 255-527.

7. Knorr, R., A. Trzeciak, W. Bannwarth, et al., New coupling reagents in peptide chemistry. Tetrahedron Letters, 1989, 30(15): p. 1927-1930.

8. Shapira, R., F.C. Chou, R.K. Chawla, et al., Incomplete deblocking as a cause of failure sequence in solid phase peptide synthesis. Journal of the American Chemical Society, 1971, 93(1): p. 267-268.

9. Nogueira, R., M. Lämmerhofer, and W. Lindner, Alternative highperformance liquid chromatographic peptide separation and purification 
concept using a new mixed-mode reversed-phase/weak anion-exchange type stationary phase. Journal of Chromatography A, 2005, 1089(1): p. 158-169. 


\title{
Chapter 6
}

\section{Purification of Synthetic Peptides Using a Catching Full-Length Sequence by Polymerization Approach ${ }^{1}$}

\author{
Mingcui Zhang, Durga Pokharel, and Shiyue Fang \\ Department of Chemistry, Michigan Technological University, 1400 Townsend \\ Drive, Houghton, MI 49931 USA \\ Email: shifang@mtu.edu
}

\begin{abstract}
${ }^{1}$ The material in this chapter was previously published in Organic Letters, 2014, 16, 1290-1293. DOI: $10.1021 / \mathrm{ol} 403426 u$

http://pubs.acs.org/doi/abs/10.1021/ol403426u
\end{abstract}


The idea of the project in chapter 6 was conceived by Dr. Shiyue Fang. Dr. Fang also revised writing of Durga P. Pokharel. More than $60 \%$ of experiments in "Purification of Synthetic Peptides Using a Catching Full-Length Sequence by Polymerization Approach" were carried out by Durga P. Pokharel. Dr. Mingcui Zhang did $40 \%$ of experiments. At the beginning during the first submission for publication, Dr. Mingcui Zhang had more contribution than Durga P. Pokharel. At re-submission, we added more data the reviewers requested. At that stage, the contribution of Durga became more than Dr. Mingcui. At that time, we did not change authorship so Dr. Mingcui Zhang is first author in chapter 6

\section{Apporved by advisor Dr. Shiyue Fang}




\begin{abstract}
During automated solid phase peptide synthesis, failure sequences were capped with acetic anhydride. After synthesis, a polymerizable methacrylamide tag was attached to the full-length sequences. Peptide purification was then achieved by polymerizing the full-length sequences, washing away impurities, and cleaving the peptide product from the polymer
\end{abstract}




\subsection{Introduction}

Synthetic peptides have wide applications. For example, there are 65 therapeutic peptides on the market and around 270 peptides in clinical trials.[1, 2] Peptides are mostly synthesized on a solid support by stepwise addition of amino-protected amino acid (aa) monomers. Each synthetic cycle accomplishes the addition of one aa. It usually consists of three steps, which are coupling, capping and deprotection. Excess reagents and side products in each step are removed by washing. After synthesis, the product is cleaved from the support and fully deprotected. Besides the desired fulllength peptide, the major impurities in the crude product include failure sequences and small molecules from side chain protecting groups. The latter can usually be removed by precipitation with cold diethyl ether from a trifluoroacetic acid (TFA) solution. However, the failure sequences have physical properties similar to those of full-length peptide, and cannot be removed in the process.

Currently, the first industrial choice for peptide purification is reversed-phase (RP) HPLC. However, it has limitations. For example, in the manufacture of enfuvirtide, which is a 36-aa peptide used to treat HIV infection, RP HPLC is the primary tool for purification. When a linear solid phase synthesis method was used, the purity of the crude product was $30-40 \%$, which is acceptable considering the length of the synthesis and the convenience of the procedure. Unfortunately, the product had to be purified by a complicated procedure involving difficult, low-concentration, low- 
throughput, and two-pass HPLC.[3] To ease purification, later a combined solid phase and solution phase synthesis method was developed, and the purity of product was improved to $\sim 75 \%$. However, the purification was not much easier. The product was loaded in multiple portions onto a column for preparative HPLC.[3] In general, the disadvantages of HPLC for large scale purification include high capital costs on instrument and column, labor intensiveness, high energy demand for solvent evaporation, and inability to resolve peptides with multiple higher order structures. Most importantly, HPLC consumes large volumes of harmful solvents, which results in high waste to product ratios, usually more than 1,000 . Other methods for peptide purification include fluorous affinity purification,[4-10] antigen-antibody affinity purification,[11, 12] covalent capture with a solid matrix,[13-15] and lipophilic tag assisted chromatography.[16] All these methods are extensively studied, but are still not ideal for large scale peptide drug purification.

\subsection{Results and Discussion}

Recently, we reported a catching by polymerization strategy for purification of oligodeoxynucleotides (ODN).[17-19] In one method, at the end of automated synthesis, a polymerizable methacrylamide group is attached to the full-length sequence. ODN purification is then achieved by polymerizing the full-length sequences, washing away failure sequences and other impurities, and cleaving product from the polymer.[17] The method avoided many drawbacks associated with chromatography and other known methods, and could be used for large scale and high 
throughput ODN purification. Here we report our results on using the strategy for synthetic peptide purification.

To demonstrate feasibility, compound 6.1, which contains an acid-labile linker and a reactive 4-nitrophenyl carbonate function, was designed. Because we planned to use the commercially available 2-Cl-trityl polystyrene resin as the solid support for peptide synthesis, the linker must be stable in $1 \%$ TFA, which are the conditions for cleaving peptides from the resin.[3, 20-23] However, the linker must be readily cleavable under more acidic conditions to obtain good recovery yield in the purification process. These goals were achieved by careful tuning the electron density of the benzene ring in the linker. The molecule was also designed to be easy to synthesize. As shown in Scheme 1, 4-hydroxybenzyl alcohol was alkylated with methyl chloroacetate to give $\mathbf{6 . 2},[24]$ which was reacted at room temperature with 2,2'-(ethylenedioxy)bis(ethylamine) to give 6.3.[25] Methacrylation of $\mathbf{6 . 3}$ gave compound 6.4, which was converted to $\mathbf{6 . 1}$ by reacting with 4nitrophenylchloroformate. The synthesis did not use any expensive and highly reactive reagents, and is expected to be readily scalable. 


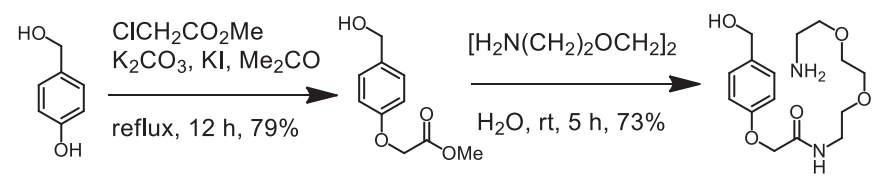

6.2

6.3

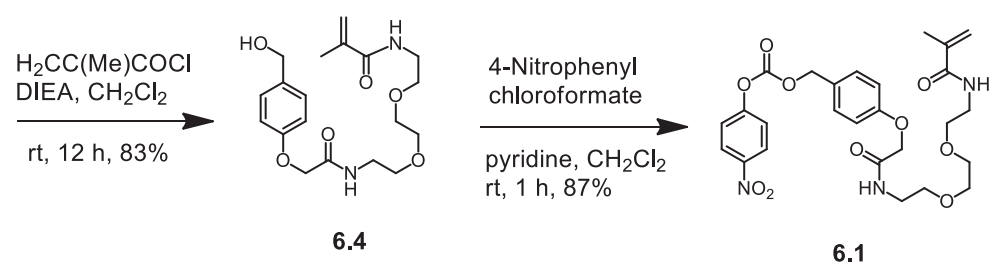

Scheme 6.1. Synthesis of compound 6.1

Since peptide synthesis and purification are much more complicated than ODN, at the beginning of the project, we used the short 6-aa peptide 6.5 (LWTRFA) to demonstrate the feasibility of the concept. The 2-Cl-trityl polystyrene resin preloaded with Ala was selected as the solid support. The Fmoc-protected Phe, $\operatorname{Arg}(\mathrm{Pbf}), \operatorname{Thr}(t \mathrm{Bu}), \operatorname{Trp}(\mathrm{Boc})$ and Leu were used as aa monomers. Synthesis was carried out on an automated synthesizer. In the coupling steps, only 2.1 equivalents of monomers were used, which was lower than the amounts usually suggested by manufacturers of peptide synthesizers. The lowered amount was expected to generate more failure sequences and to better show the effectiveness of the new purification method. After coupling, failure sequences were capped with excess acetic anhydride. The steps for removing Fmoc protecting group were normal. After assembling the sequence, the full-length peptide was reacted with compound 6.1 in the presence of a base at room temperature overnight to give $\mathbf{6 . 6}$ (see Scheme 6.2). The failure sequences (6.7) could not react because they were capped with acetic anhydride. The 
crude product, which mainly contained 6.6 and 6.7 , were cleaved from the resin using 1\% TFA to give full-length peptide $\mathbf{6 . 8}$ and failure sequences 6.9. Under these conditions, the side chain protecting groups and the 4-hydroxybenzyl alcohol linker were stable.[3] The mixture was analysed with RP HPLC (trace a, Figure 6.1). The full-length peptide appeared at 61 minutes. To generate a control HPLC profile, a portion of the crude was treated with a TFA cocktail containing $81.5 \%$ TFA, 1.0\% triisopropylsilane (TIPS), 5.0\% water, 2.5\% ethane dithiol (EDT), 5.0\% thioanisole, and $5.0 \%$ phenol to globally remove protecting groups and to cleave the methacrylamide tag. After $\mathrm{Et}_{2} \mathrm{O}$ precipitation, the crude product was analysed with RP HPLC (trace b). The fully deprotected full-length peptide (6.5) appeared at 23 minutes. 

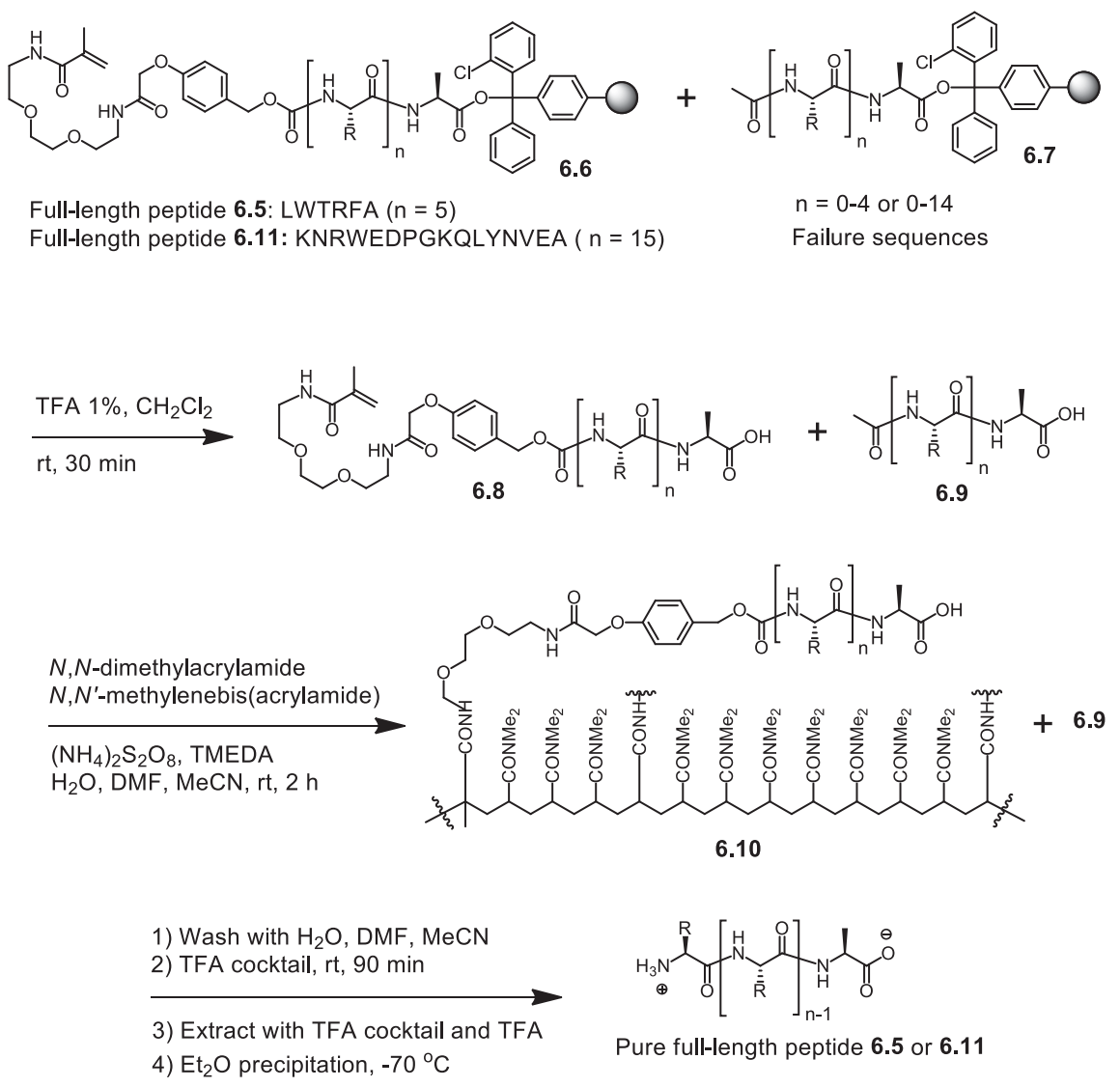

Pure full-length peptide $\mathbf{6 . 5}$ or $\mathbf{6 . 1 1}$

Scheme 6.2. Peptide cleavage from resin and purification by catching full-length sequence by polymerization

Another portion of the crude containing 6.8 and 6.9 (with side chain protecting groups and methacrylamide tag not removed) was subjected to polymerization under typical polyacrylamide gel formation conditions $[N, N-$ dimethylacrylamide, $N, N^{\prime}$-methylenebis(acrylamide), $\left(\mathrm{NH}_{4}\right)_{2} \mathrm{~S}_{2} \mathrm{O}_{8}$, TMEDA]. The full-length peptide 6.8 was incorporated into the polymer 6.10. The failure sequences 6.9 and other impurities remained in solution (Scheme 6.2). The gel was washed 
extensively with solvents such as water, DMF and $\mathrm{MeCN}$ to remove impurities. Fulllength peptide was then cleaved from the gel and fully deprotected by
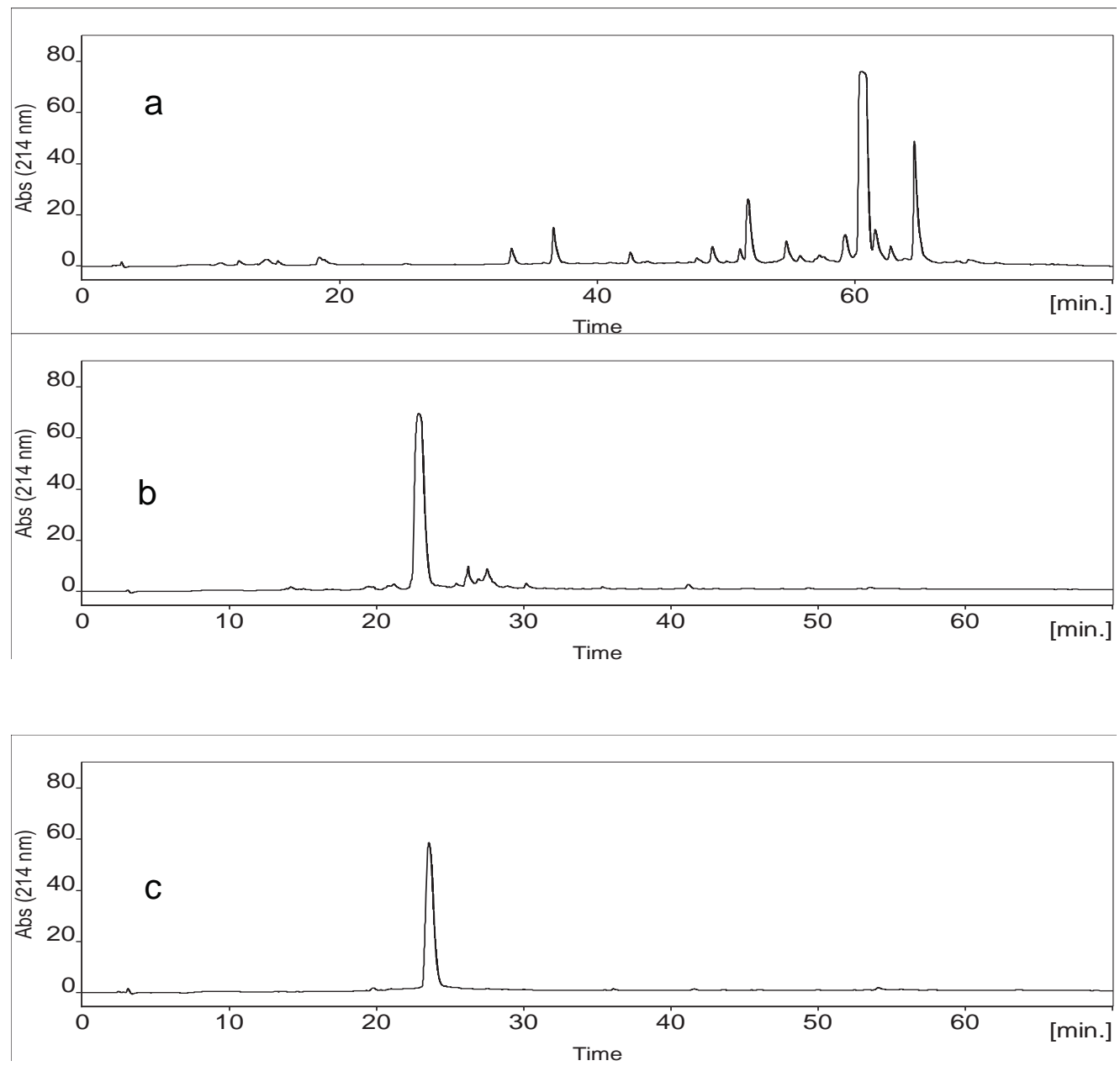

Figure 6.1. RP HPLC profiles of peptides. (a) Crude 6-aa peptide containing fulllength sequences 6.8 and failure sequences 6.9 , the side chain protecting groups and the methacrylamide tag on $\mathbf{6 . 8}$ were on; (b) crude peptide with side chain protecting groups and methacrylamide tag removed; (c) peptide 6.5 purified using the catching full-length sequences by polymerization approach. 
treating with the TFA cocktail described above. At this stage, the peptide was still contaminated with small molecules from side chain protecting groups and the cocktail. Removing them was achieved by precipitation of peptide with cold $\mathrm{Et}_{2} \mathrm{O}$. The pure peptide 6.5 was analysed with RP HPLC (trace c, Figure 6.1). The purity of the peptide was more than $98 \%$. The recovery yield of the purification process was estimated to be $66 \%$ by comparing the areas of the peaks at 23 minutes in traces $b$ and c. The identity of the peptide was confirmed with ESI-MS and NMR (see Supporting Information).

To further demonstrate the effectiveness of the purification technique, the 16-aa peptide 6.11 (KNRWEDPGKQLYNVEA), which is a peptide derived from human C3d and carries the LYNVEA CR2 binding sequence,[26] was synthesized under similar conditions described for 6.5. Fmoc aa $\mathrm{Glu}(\mathrm{O} t \mathrm{Bu})$, Val, $\mathrm{Asn}(\operatorname{Trt}), \operatorname{Tyr}(t \mathrm{Bu})$, Leu, Gln(Trt), Lys(Boc), Gly, Pro, $\operatorname{Asp}(\mathrm{O} t \mathrm{Bu}), \operatorname{Trp}(\mathrm{Boc})$ and $\operatorname{Arg}(\mathrm{Pbf})$ were used as the monomers. To further increase the difficulty of the purification task, the amount of aa monomers was lowered to 1.5 equivalents. The purification process was exactly the same. The RP HPLC profiles for the crude peptide with the methacrylamide tag and side chain protecting groups on, that with tag and protecting groups removed, and purified full-length peptide are included in Supporting Information. The purity of the peptide (6.11) is more than $96 \%$. The recovery yield was estimated to be $73 \%$. The identity of 6.11 was confirmed with ESI MS and NMR.

The catching by polymerization peptide purification method has significant advantages over other techniques. Compared with chromatographic methods such as 
HPLC and lipophilic tag assisted purification,[16] the method does not need any expensive instrument, column, or large volumes of harmful solvents. Compared with RP cartridge, fluorous and antigen-antibody affinity purification methods,[4-12] the method does not require a disposable column. Compared with the methods using covalent capture,[13-15] the method does not need a functionalized solid matrix. Importantly, using the method, purification is achieved by simple manipulations such as shaking, washing, extraction and precipitation. As a result, it is expected to be particularly useful for large scale peptide drug purification. Some drugs need to be purified for more than one time, and ideally, each time uses a different purification technique. The catching by polymerization method may be an excellent choice for initial removal of large quantities of failure sequences. This is predicted to be particularly useful for the purification of peptide sequences that have inherently low coupling yields in certain cycles. Furthermore, due to the ease of removal of failure sequences using the method, it is possible for drug manufacturers to reduce the equivalents of aa monomers and their activators during synthesis. The re-balance of reagent costs and purification costs will provide new opportunities to lower the overall expenses of drug production.

\subsection{Experimental Section}

\section{General Experimental}

All reactions were performed in oven-dried glassware under a nitrogen atmosphere using standard Schlenk techniques. Reagents and solvents available from commercial sources were used as received unless otherwise noted. Pyridine and 
$\mathrm{CH}_{2} \mathrm{Cl}_{2}$ were distilled over $\mathrm{CaH}_{2}$ under nitrogen. Thin layer chromatography (TLC) was performed using plates with silica gel 60F-254 over glass support, $0.25 \mu \mathrm{m}$ thickness. Flash column chromatography was performed using silica gel with particle size $32-63 \mu \mathrm{m} .{ }^{1} \mathrm{H}$ and ${ }^{13} \mathrm{C}$ NMR spectra were measured at 400 and $100 \mathrm{MHz}$, respectively on a Varian $400 \mathrm{MHz}$ spectrometer. Chemical shifts $(\delta)$ were reported in reference to solvent peaks (residue $\mathrm{CHCl}_{3}$ at $\delta 7.24 \mathrm{ppm}$ for ${ }^{1} \mathrm{H}$ and $\mathrm{CDCl}_{3}$ at $\delta 77.00$ ppm for ${ }^{13} \mathrm{C}$, and residue $\mathrm{CHD}_{2} \mathrm{OD}$ at $\delta 3.31 \mathrm{ppm}$ for ${ }^{1} \mathrm{H}$ and $\mathrm{CD}_{3} \mathrm{OD}$ at $\delta 49.00 \mathrm{ppm}$ for ${ }^{13} \mathrm{C}$ ). ESI-MS for small molecules were obtained on an Agilent Q-TOF System; those for peptides were obtained on a Thermo Finnigan LCQ Advantage spectrometer. H-Ala-2-Cl-Trt polystyrene resin (1\% DVB cross-linking, 100-200 mesh, $\sim 0.56 \mathrm{mmol} / \mathrm{g}$ loading for the synthesis of 6.5 and $\sim 0.80 \mathrm{mmol} / \mathrm{g}$ for $\mathbf{6 . 1 1}$ ), Fmoc protected amino acids, $N$-hydroxybenzotriazole $\left(\mathrm{HOBt} \cdot \mathrm{H}_{2} \mathrm{O}\right), \quad N, N^{\prime}-$ diisopropylcarbodiimide (DIC), piperidine, and $N, N$-diisopropylethylamine(DIEA) were purchased from AAPPTec (Louisville, USA). RP HPLC was performed on a JASCO LC-2000Plus System: pump, PU-2089Plus Quaternary Gradient; detector UV-2075Plus. RP analytical columns XBridge ${ }^{\mathrm{TM}}, \mathrm{C} 18,5.0 \mu \mathrm{m}$ diameter, $3.0 \mathrm{~mm} \times$ $250 \mathrm{~mm}$ (for 6-amino acid peptide 6.5); and XBridge ${ }^{\mathrm{TM}}, \mathrm{C} 18,5.0 \mu \mathrm{m}$ diameter, 4.6 $\mathrm{mm} \times 150 \mathrm{~mm}$ (for 16 -amino acid peptide 6.11) were used. Solvent A: $0.05 \%$ trifluoroacetic acid (TFA) in water. Solvent B: 0.05\% TFA in acetonitrile. All profiles were generated by detection of absorbance of peptide at $214 \mathrm{~nm}$ using the linear gradient solvent system: solvent B (0\%-15\%) in solvent A over 9 min followed by solvent B (15\%-100\%) in solvent A over $71 \mathrm{~min}$ at flow rates of $1.0 \mathrm{~mL} / \mathrm{min}(4.6 \mathrm{~mm}$ $\times 150 \mathrm{~mm}$ column $)$ and $0.5 \mathrm{~mL} / \mathrm{min}(3.0 \mathrm{~mm} \times 250 \mathrm{~mm}$ column$)$. 


\section{Synthesis and Characterization of Compounds}

\section{Compound 6.3}

To a round-bottomed flask under a nitrogen atmosphere was added $\mathbf{6 . 2}$ (2.48 g, 12.64 mmol, 1.0 equiv.), water (1 mL) and 2,2'-(ethylenedioxy)bis(ethylamine) (5.6 mL, $38 \mathrm{mmol}, 3.0$ equiv.). The reaction mixture was stirred for $5 \mathrm{~h}$ at $\mathrm{rt}$. The crude was loaded onto a flash chromatography column $\left(\mathrm{SiO}_{2}\right)$. Eluting with the solvent mixture $\mathrm{Et}_{2} \mathrm{O} / \mathrm{MeOH} / \mathrm{MeCN} / \mathrm{Et}_{3} \mathrm{~N}(\mathrm{v} / \mathrm{v} / \mathrm{v} / \mathrm{v}$ 5:2:2:1) gave 6.3 as a sticky solid $(2.78 \mathrm{~g}, 8.89 \mathrm{mmol}, 70 \%): R_{\mathrm{f}}=0.32\left(\mathrm{Et}_{2} \mathrm{O} / \mathrm{MeOH} / \mathrm{MeCN} / \mathrm{Et}_{3} \mathrm{~N}, 5: 2: 2: 1\right) ;{ }^{1} \mathrm{H} \mathrm{NMR}$ (400 MHz, CD $\left.{ }_{3} \mathrm{OD}\right) \delta 7.28(\mathrm{~d}, J=8.4 \mathrm{~Hz}, 2 \mathrm{H}), 6.94(\mathrm{~d}, J=8.4 \mathrm{~Hz}, 2 \mathrm{H}), 4.51(\mathrm{~s}, 2 \mathrm{H})$, $4.47(\mathrm{~s}, 2 \mathrm{H}), 3.65-3.43(\mathrm{~m}, 10 \mathrm{H}), 2.75(\mathrm{t}, J=5.2 \mathrm{~Hz}, 2 \mathrm{H}) ;{ }^{13} \mathrm{C}$ NMR $(100 \mathrm{MHz}$, $\left.\mathrm{CD}_{3} \mathrm{OD}\right) \delta 169.9,157.3,135.1,128.5,114.6,72.4,70.1,69.2,67.2,63.5,40.8,38.8$ HRMS (ESI) calcd for $\mathrm{C}_{15} \mathrm{H}_{25} \mathrm{~N}_{2} \mathrm{O}_{5}[\mathrm{M}+\mathrm{H}]^{+} m / z$ 313.1763, found 313.1762.

\section{Compound 6.4}

To a round-bottomed flask under a nitrogen atmosphere was added 6.3 (4.5 g, 14.41 mmol, 1.0 equiv.), freshly distilled $\mathrm{CH}_{2} \mathrm{Cl}_{2} \quad(100 \mathrm{~mL}), \quad N, N-$ diisopropylethylamine (7.5 mL, $43.23 \mathrm{mmol}, 3.0$ equiv.), and methacrolyl chloride (1.34 mL, $13.69 \mathrm{mmol}, 0.95$ equiv.). The reaction mixture was stirred at $\mathrm{rt}$ overnight. The contents were transferred into a separatory funnel and extracted with EtOAc (50 $\mathrm{mL} \times 3$ ). The combined organic phase was dried over anhydrous $\mathrm{Na}_{2} \mathrm{SO}_{4}$, and filtered. Volatiles were removed under reduced pressure. The crude product was purified by flash column chromatography $\left(\mathrm{SiO}_{2}\right.$, EtOAc/acetone, 7:3) giving 6.4 as a 
light yellow oil $(4.53 \mathrm{~g}, 11.91 \mathrm{mmol}, 82 \%): R_{\mathrm{f}}=0.24($ EtOAc/acetone, $7: 3) ;{ }^{1} \mathrm{H}$ NMR $\left(400 \mathrm{MHz}, \mathrm{CDCl}_{3}\right) \delta 7.25(\mathrm{~d}, J=8.8 \mathrm{~Hz}, 2 \mathrm{H}), 6.95(\mathrm{br} \mathrm{s}, 1 \mathrm{H}), 6.82(\mathrm{~d}, J=8.8 \mathrm{~Hz}$, 2H), 6.36 (br s, 1H), 5.63 (s, 1H), 5.26 (s, 1H), $4.56(\mathrm{~s}, 2 \mathrm{H}), 4.39$ (s, 2H), 3.59-3.36 (m, 12H), 2.88 (br s 1H), 1.88 (s, 3H); ${ }^{13} \mathrm{C}$ NMR $\left(100 \mathrm{MHz}, \mathrm{CDCl}_{3}\right) \delta 168.7$, 168.6, $156.8,140.1,135.4,128.8,119.8,114.8,70.4,70.3,69.9,69.8,67.7,64.6,39.5,38.9$, 18.8; HRMS (ESI) calcd for $\mathrm{C}_{19} \mathrm{H}_{28} \mathrm{~N}_{2} \mathrm{NaO}_{6}[\mathrm{M}+\mathrm{Na}]^{+} \mathrm{m} / z$ 403.1845, found 403.1844.

\section{Compound 6.1}

To a round-bottomed flask under a nitrogen atmosphere was added $6.4(1.1 \mathrm{~g}$, 2.89 mmol, 1.0 equiv.), freshly distilled $\mathrm{CH}_{2} \mathrm{Cl}_{2}(100 \mathrm{~mL})$, freshly distilled pyridine (1.16 mL, $14.45 \mathrm{mmol}, 5.0$ equiv.), and 4-nitrophenyl chloroformate (728.2 mg, 3.61 mmol, 1.25 equiv.). The reaction mixture was stirred at $\mathrm{rt}$ for $1 \mathrm{~h}$. The contents were transferred into a separatory funnel and partitioned between sat. $\mathrm{NH}_{4} \mathrm{Cl}$ and $\mathrm{CH}_{2} \mathrm{Cl}_{2}$ (50 mL). The aqueous phase was further extracted with $\mathrm{CH}_{2} \mathrm{Cl}_{2}(50 \mathrm{~mL} \times 2)$. The combined organic phase was dried over anhydrous $\mathrm{Na}_{2} \mathrm{SO}_{4}$ and filtered. Volatiles were removed under reduced pressure. The crude product was purified by flash column chromatography $\left(\mathrm{SiO}_{2}\right.$, EtOAc/acetone, 7:3) giving 6.1 as a light yellow solid $(1.37 \mathrm{~g}, 2.51 \mathrm{mmol} 86 \%): R_{\mathrm{f}}=0.37$ (EtOAc/acetone, 7:3); m.p. $81.2{ }^{\circ} \mathrm{C} ;{ }^{1} \mathrm{H}$ NMR $\left(400 \mathrm{MHz}, \mathrm{CDCl}_{3}\right) \delta 8.24(\mathrm{~d}, J=9.2 \mathrm{~Hz}, 2 \mathrm{H}), 7.42-7.32(\mathrm{~m}, 4 \mathrm{H}), 6.92(\mathrm{~d}, J=8.8 \mathrm{~Hz}$, 2H), 6.25 (br s, 1H), $5.66(\mathrm{~s}, 1 \mathrm{H}), 5.28(\mathrm{~s}, 1 \mathrm{H}), 5.21(\mathrm{~s}, 2 \mathrm{H}), 4.48(\mathrm{~s}, 2 \mathrm{H}), 3.58-3.46$ (m, 12H), $1.91(\mathrm{~s}, 3 \mathrm{H}) ;{ }^{13} \mathrm{C}$ NMR $\left(100 \mathrm{MHz}, \mathrm{CDCl}_{3}\right) \delta$ 168.6, 168.1, 157.9, 155.7, 152.6, 145.6, 140.2, 131.1, 128.1, 125.5, 121.9, 119.8, 115.2, 70.7, 70.5, 70.4, 70.0, 
69.9, 67.6, 39.5, 38.9, 18.8; HRMS (ESI) calcd for $\mathrm{C}_{26} \mathrm{H}_{31} \mathrm{~N}_{3} \mathrm{NaO}_{10}[\mathrm{M}+\mathrm{Na}]^{+} \mathrm{m} / \mathrm{z}$ 568.1907, found 568.1903.

\section{Peptide Synthesis, Acrylation and Cleavage}

\section{Peptide Synthesis:}

The 6-amino acid peptide $6.5\left(\mathrm{H}_{2} \mathrm{~N}-\mathrm{LWTRFA}-\mathrm{CO}_{2} \mathrm{H}\right)$ was synthesized on an automated solid phase synthesizer using the Fmoc protecting strategy. The 2-ChloroTrityl-Ala-H polystyrene resin (with $1 \%$ DVB cross-linking, $0.56 \mathrm{mmol} / \mathrm{g}$ loading, $0.39 \mathrm{mmol}, 0.70 \mathrm{~g})$ was swollen by soaking in $\mathrm{CH}_{2} \mathrm{Cl}_{2}(25 \mathrm{~mL})$ at $\mathrm{rt}$ for $45 \mathrm{~min}$. The solvent was removed. The resin was washed with DMF for 3 times and finally the DMF was removed. Coupling was then carried out. To the amino acid tube on the synthesizer containing Fmoc-Phe- $\mathrm{OH}(2.1 \mathrm{eq}, 0.84 \mathrm{mmol}, 0.32 \mathrm{~g})$ and $\mathrm{HOBt} \cdot \mathrm{H}_{2} \mathrm{O}$ (2.1 eq, $0.84 \mathrm{mmol}, 0.13 \mathrm{~g}$ ) was added DMF $(3.5 \mathrm{~mL})$. The mixture was dissolved by bubbling nitrogen. After $5 \mathrm{~min}$, DIC (2.1 eq, $0.84 \mathrm{mmol})$ in $3.5 \mathrm{~mL}$ DMF was added. Pre-activation of the amino acid was proceeded at $\mathrm{rt}$ for $20 \mathrm{~min}$. The mixture containing the activated ester was added to the reaction flask containing the resin. Coupling was allowed to proceed at $\mathrm{rt}$ for $60 \mathrm{~min}$. The reagents were removed. The resin was washed with DMF $(10 \mathrm{~mL} \times 4)$. Capping failure sequences was then performed. First a solution of DIEA (1.5 M) in DMF, and then the solution of $\mathrm{Ac}_{2} \mathrm{O}$ $(1.0 \mathrm{M})$ also in DMF were delivered to the reaction flask. The ratio of the volumes of the two solutions was approximately $1: 1$, and the total volume of the two solutions was approximately $10 \mathrm{~mL}$, which could cover the entire resin. The mixture was allowed to react at $\mathrm{rt}$ for $30 \mathrm{~min}$. The reagents were removed and the resin was 
washed with $\mathrm{DMF}(10 \mathrm{~mL} \times 2)$. The process was repeated for three more times under the same conditions with the exception of reducing the capping time from $30 \mathrm{~min}$ to $15 \mathrm{~min}$. The resin was washed with DMF $(10 \mathrm{~mL} \times 4)$. Removal of Fmoc group was

then carried out. A solution of piperidine in DMF (20\%, $10 \mathrm{~mL})$ was delivered to the reaction flask, and the mixture was allowed to react at $\mathrm{rt}$ for $3 \mathrm{~min}$. The reagents were removed. The process was repeated for two more times. The resin was washed with DMF $(10 \mathrm{~mL} \times 4$ times). This completed one synthesis cycle, in which one amino acid was added to the elongating peptide. The synthesis cycle was repeated with the Fmoc amino acid monomers $\operatorname{Arg}(\mathrm{Pbf}), \operatorname{Thr}(\mathrm{tBu}), \operatorname{Trp}(\mathrm{Boc})$ (120 min for coupling), and Leu sequentially. At the end of the synthesis, the Fmoc group was removed.

\section{Acrylation of the Full-Length Peptide}

The solutions of compound 6.1 (3.1 eq, $1.23 \mathrm{mmol}, 0.67 \mathrm{~g})$ in DMF (2.0 mL), and DIEA (10\%) in DMF $(2.0 \mathrm{~mL})$ were combined, and then added to the resin manually via a pipette. The reaction was allowed to proceed at $\mathrm{rt}$ overnight. The resin was filtered, and washed with DMF $(2.0 \mathrm{~mL} \times 4)$ and $\mathrm{CH}_{2} \mathrm{Cl}_{2}(2.0 \mathrm{~mL} \times 4)$, and then dried under vacuum. The thoroughly dried resin $(0.95 \mathrm{~g})$ was stored at $-20{ }^{\circ} \mathrm{C}$ under inert atmosphere.

\section{Cleaving the Peptide From the Resin and HPLC Analysis of Crude Peptide}

A portion of the resin $(0.12 \mathrm{~g})$ was swollen in $\mathrm{CH}_{2} \mathrm{Cl}_{2}(3.0 \mathrm{~mL})$ at $\mathrm{rt}$ for 45 min. The solvent was removed with a pipette, and the resin was treated with a solution of $1 \%$ TFA in $\mathrm{CH}_{2} \mathrm{Cl}_{2}(1.0 \mathrm{~mL})$ at $\mathrm{rt}$. After $30 \mathrm{~min}$, the mixture was filtered. 
The resin was washed with $1 \%$ TFA in $\mathrm{CH}_{2} \mathrm{Cl}_{2}(200 \mu \mathrm{L} \times 2)$. To the combined solution was added $\mathrm{Et}_{2} \mathrm{O}(12 \mathrm{~mL})$. The mixture was vortexed and spun briefly to bring down contents to the bottom of the tube, and cooled to $-70{ }^{\circ} \mathrm{C}$. After $1 \mathrm{~h}$, the mixture was centrifuged for $3 \min \left(\right.$ at $\sim 10{ }^{\circ} \mathrm{C}$ ). The supernatant was removed with a pipette. The residue was washed with cold $\mathrm{Et}_{2} \mathrm{O}\left(1 \mathrm{~mL} \times 3,-70{ }^{\circ} \mathrm{C}\right)$. After washing, residue $\mathrm{Et}_{2} \mathrm{O}$ was allowed to evaporate in air. The crude product $(26.7 \mathrm{mg})$, which contained the full-length sequences 6.8, failure sequences 6.9 and other impurities, was obtained as an amorphous solid. A portion (10 mg) was dissolved in $60 \%$ $\mathrm{CH}_{3} \mathrm{CN}(1.0 \mathrm{~mL})$. From the solution, $20 \mu \mathrm{L}$ was injected into RP HPLC to generate trace a (Figure 6.1). From the remaining solution $(980 \mu \mathrm{L})$ was drawn $100 \mu \mathrm{L}$, and evaporated to dryness in a centrifugal vacuum concentrator. The residue was treated with a TFA cocktail $(100 \mu \mathrm{L})$, which contained $81.5 \%$ TFA, $1.0 \%$ triisopropylsilane (TIPS), $5.0 \%$ water, $2.5 \%$ ethane dithiol (EDT), $5.0 \%$ thioanisole, and $5.0 \%$ phenol, at $\mathrm{rt}$ for $100 \mathrm{~min}$. To the solution was added $\mathrm{Et}_{2} \mathrm{O}(900 \mu \mathrm{L})$. The mixture was vortexed and spun briefly, and cooled to $-70{ }^{\circ} \mathrm{C}$. After $1 \mathrm{~h}$, the mixture was centrifuged for $3 \min \left(\right.$ at $\left.\sim 10^{\circ} \mathrm{C}\right)$. The supernatant was removed with a pipette. The residue was washed with cold $\mathrm{Et}_{2} \mathrm{O}\left(1 \mathrm{~mL} \times 3,-70{ }^{\circ} \mathrm{C}\right)$. Residue $^{\mathrm{E}} \mathrm{t}_{2} \mathrm{O}$ was allowed to evaporate in air. The solid was dissolved in $100 \mu \mathrm{L} 50 \% \mathrm{CH}_{3} \mathrm{CN}, 20 \mu \mathrm{L}$ was injected into RP HPLC to generate trace b (Figure 6.1). 


\section{Purification by Catching Full-Length Sequences by Polymerization}

\section{Catching Full-Length Peptide by Polymerization}

Purification was carried out with the side chain protected peptide to avoid side reactions between peptide and acrylamide. An additional benefit is that protected peptide must be soluble at least in the solvent in which it is synthesized. This will ensure that other peptides could be purified using the method. Thus, from the above remaining solution $(\sim 880 \mu \mathrm{L})$ of crude peptide containing 6.8 and 6.9 with side chain protected, $100 \mu \mathrm{L}$ was transferred into a $25 \mathrm{~mL}$ 2-necked round bottomed flask. To the same flask, $150 \mu \mathrm{L} \mathrm{CH}_{3} \mathrm{CN}, 270 \mu \mathrm{L}$ water, and $520 \mu \mathrm{L}$ polymerization solution,

which contained $N, N$-dimethylacrylamide $(1.69 \quad \mathrm{M}) \quad$ and $\quad N, N^{\prime}-$ methylenebis(acrylamide) (0.068 M) in DMF, were added. The mixture was stirred using a magnetic stirring bar while a gentle nitrogen flow passed over the liquid surface. After $5 \mathrm{~min}$, a solution of $\left(\mathrm{NH}_{4}\right)_{2} \mathrm{~S}_{2} \mathrm{O}_{8}(10 \%, 5 \mu \mathrm{L})$ and TMEDA $(3.5 \mu \mathrm{L})$ were added sequentially via a pipette and a syringe, respectively, under positive nitrogen pressure. The solution was stirred gently under nitrogen at $\mathrm{rt}$ for $1 \mathrm{~min}$ to mix the materials, and then stirring was stopped. The mixture was allowed to stand under nitrogen. The gel $\mathbf{6 . 1 0}$ was formed within 30 min. To ensure complete polymerization, the gel was left under nitrogen for $2 \mathrm{~h}$ or longer.

\section{Removing Failure Sequences and Other Impurities by Washing}

To the flask containing the gel was added $3 \mathrm{~mL}$ water via a pipette. The flask was moved onto an orbital shaker and shaken at $\mathrm{rt}$ for $2 \mathrm{~h}$. The supernatant was 
removed with a pipette. The gel was further washed with water $(3 \mathrm{~mL} \times 2,2 \mathrm{~h}$ each time), DMF ( $3 \mathrm{~mL} \times 3,2 \mathrm{~h}$ each time), $50 \% \mathrm{CH}_{3} \mathrm{CN}(3 \mathrm{~mL} \times 3,2 \mathrm{~h}$ each time), and $\mathrm{CH}_{3} \mathrm{CN}$ ( $3 \mathrm{~mL} \times 3,2 \mathrm{~h}$ each time) to ensure complete removal of failure sequences and other impurities. The gel 6.10 was then dried under vacuum.

\section{Cleaving Full-Length Peptide From Gel and Global Peptide Deprotection}

To the flask containing gel $\mathbf{6 . 1 0}$ was added $2.0 \mathrm{~mL}$ TFA cocktail (81.5\% TFA, 1.0\% TIPS, 5.0\% water, 2.5\% EDT, 5.0\% thioanisole, and 5.0\% phenol) via a pipette. The flask was gently shaken at rt for $100 \mathrm{~min}$. The supernatant, which contained the full-length peptide 6.5 and small molecules from side chain deprotection, was transferred to a $45 \mathrm{~mL}$ centrifuge tube using a pipette. The gel was further washed with the TFA cocktail $(2.0 \mathrm{~mL}, 1 \mathrm{~h})$, pure TFA $(2.0 \mathrm{~mL}, 2 \mathrm{~h} ; 1.0 \mathrm{~mL} \times 3,1 \mathrm{~h}$ each time) to ensure complete extraction of full-length sequences. The last extract was concentrated and precipitated with $\mathrm{Et}_{2} \mathrm{O}$ at $-70{ }^{\circ} \mathrm{C}$, and analyzed with HPLC. No peptide was detected, which indicates that the extraction process could be simplified. All other extracts were combined into the same $45 \mathrm{~mL}$ centrifuge tube, and concentrated to about $2.5 \mathrm{~mL}$ with a flow of nitrogen. To the solution was added $\mathrm{Et}_{2} \mathrm{O}$ (22.5 mL). The mixture was vortexed and spun briefly to bring down contents to the bottom of the tube, and cooled to $-70{ }^{\circ} \mathrm{C}$. After $1 \mathrm{~h}$, the mixture was centrifuged for 3 min $\left(\right.$ at $\sim 10^{\circ} \mathrm{C}$ ). The supernatant was removed with a pipette. The residue was washed with cold $\mathrm{Et}_{2} \mathrm{O}\left(1 \mathrm{~mL} \times 3,-70{ }^{\circ} \mathrm{C}\right)$. After washing, residue $\mathrm{Et}_{2} \mathrm{O}$ was allowed to evaporate in air. The solid, which was the pure fully deprotected peptide $\mathbf{6 . 5}$, was dissolved in $100 \mu \mathrm{L} 50 \% \mathrm{CH}_{3} \mathrm{CN}, 20 \mu \mathrm{L}$ was injected into RP HPLC to generate 
trace c (Figure 6.1). Occasionally, small impurities were observed in HPLC profiles. These were caused by small molecules that were not completely removed by the precipitation process. We were able to remove them by re-dissolving the peptide in a minimum amount of $50 \% \mathrm{CH}_{3} \mathrm{CN}$ followed by evaporation in a centrifugal vacuum concentrator. The recovery yield of the catching by polymerization purification process was estimated to be $66 \%$ by comparing the area of the peak at 23 min in trace $c$ with that in trace $b$. The identity of the peptide was established with ESI-MS using a $50 \%$ acetonitrile solution, calcd for $[\mathrm{M}+\mathrm{H}]^{+} \mathrm{m} / z$ 793.9, found 793.5. The image of the spectrum is shown in this Supporting Information. To obtain sufficient peptide for NMR experiment, the purification was repeated with peptides cleaved from $26.5 \mathrm{mg}$ of resin, which theoretically contains $10.9 \mu \mathrm{mol}$ peptide. The product was obtained as a white powder $(5.5 \mathrm{mg}, 6.9 \mu \mathrm{mol}, 63 \%$ combined yield for synthesis and purification). ${ }^{1} \mathrm{H} \mathrm{NMR}\left(400 \mathrm{MHz}, 50 \% \mathrm{CD}_{3} \mathrm{CN}\right.$ in $\left.\mathrm{D}_{2} \mathrm{O}\right) \delta 7.98(\mathrm{~d}, J=7.2 \mathrm{~Hz}, 1 \mathrm{H}$, $\mathrm{H}-1$, see following structure for proton assignment), 7.79 (d, $J=8 \mathrm{~Hz}, 1 \mathrm{H}, \mathrm{H}-2)$, 7.71-7.58 (m, 6H, H-3), 7.55 (t, $J=8 \mathrm{~Hz}, 1 \mathrm{H}, \mathrm{H}-4), 7.46$ (t, $J=8 \mathrm{~Hz}, 1 \mathrm{H}, \mathrm{H}-5), 5.10$ (t, $J=7.6 \mathrm{~Hz}, 1 \mathrm{H}, \mathrm{H}-6), 4.96$ (m, 1H, H-7), 4.80-4.40 (buried in solvent peak, 2H, H8), 4.26 (t, $J=7.6 \mathrm{~Hz}, 1 \mathrm{H}, \mathrm{H}-9), 3.70-3.20$ (m, 7H, H-10), 2.20-1.85 (m, 3H, H-11), 1.78-1.63 (m, 7H, H-12), 1.37 (d, $J=6.4$ Hz, 3H, H-13), 1.30-1.20 (m, 6H, H-14). 


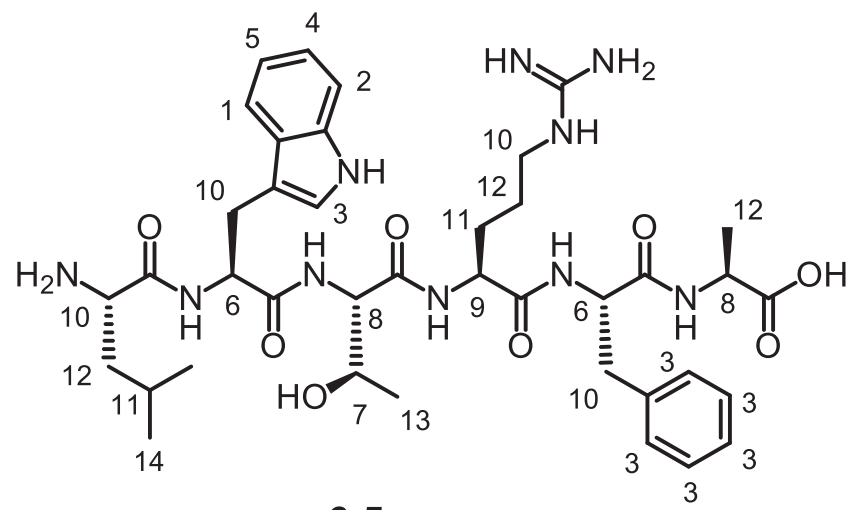

6.5

Figure 6.2. The 6-aa peptide 6.5 and proton numbering for NMR signal assignment

\section{Purification of the 16-Amino Acid Peptide 6.11}

The 16-amino acid peptide 6.11 $\left(\mathrm{H}_{2} \mathrm{~N}-\mathrm{KNRWEDPGKQLYNVEA-CO} \mathrm{C}_{2} \mathrm{H}\right)$ was synthesized using Fmoc amino acid monomers Glu(OtBu), Val, Asn(Trt), $\operatorname{Tyr}(\mathrm{tBu})$, Leu, Gln(Trt), Lys(Boc), Gly, Pro, Asp(OtBu), Trp(Boc) and $\operatorname{Arg}(\mathrm{Pbf})$, under the similar conditions on the same resin with a loading of $0.80 \mathrm{mmol} / \mathrm{g}$, and using 1.5 equivalents amino acid monomers and activating agents in the coupling steps. The catching by polymerization purification process was exactly the same as described for purification of peptide 6.5. The RP HPLC profiles for the crude peptide with the methacrylamide tag and side protecting groups on, the crude peptide with tag and protecting groups removed, and full-length peptide purified with the catching full-length sequences by polymerization approach are shown in this Supporting 
Information. The recovery yield of the purification process was estimated to be $73 \%$ by comparing the area of the peak at $17 \mathrm{~min}$ in the HPLC profiles of purified and crude peptide. The image of ESI MS for the purified peptide, calcd for $[\mathrm{M}+2 \mathrm{H}]^{2+} \mathrm{m} / \mathrm{z}$ 974.5, found 974.3, are shown in this Supporting Information. To obtain sufficient peptide for NMR experiment, the purification was repeated with peptides cleaved from $85.4 \mathrm{mg}$ of resin, which theoretically contains $12.6 \mu \mathrm{mol}$ peptide. The product was obtained as a white powder $(16.9 \mathrm{mg}, 8.7 \mu \mathrm{mol}, 69 \%$ combined yield for synthesis and purification). ${ }^{1} \mathrm{H}$ NMR $\left(400 \mathrm{MHz}, 50 \% \mathrm{CD}_{3} \mathrm{CN}\right.$ in $\left.\mathrm{D}_{2} \mathrm{O}\right) \delta 7.97(\mathrm{~d}, J=8$ $\mathrm{Hz}, 1 \mathrm{H}, \mathrm{H}-1$, see following structure for proton assignment), $7.80(\mathrm{~d}, J=8.4 \mathrm{~Hz}, 1 \mathrm{H}$, H-2), 7.60-7.40 (m, 5H, H-3), 7.13 (d, $J=8.0 \mathrm{~Hz}, 2 \mathrm{H}, \mathrm{H}-4), 5.20$ (t, $J=7.2 \mathrm{~Hz}, 1 \mathrm{H}$, H-5), 5.04 (t, $J=6.8 \mathrm{~Hz}, 2 \mathrm{H}, \mathrm{H}-5), 4.97$ (t, $J=8.4,1 \mathrm{H}, \mathrm{H}-5), 4.88-4.85$ (m, 1H, H-5), 4.75-4.44 (buried in solvent peak, 6H, H-6), 4.34 (t, $J=6.0 \mathrm{~Hz}, 1 \mathrm{H}, \mathrm{H}-6$ ), 4.26 (br s, 2H, H-7), 4.16-4.08 (m, 2H, H-6), 3.70-1.60 (m, 58H, H-8), 1.30 (d, $J=6.0 \mathrm{~Hz}, 3 \mathrm{H}$, H-9), 1.27 (d, $J=6.0 \mathrm{~Hz}, 3 \mathrm{H}, \mathrm{H}-9), 1.23$ (d, $J=6.0 \mathrm{~Hz}, 3 \mathrm{H}, \mathrm{H}-9), 1.17$ (d, $J=6.0 \mathrm{~Hz}$, 3H, H-9).

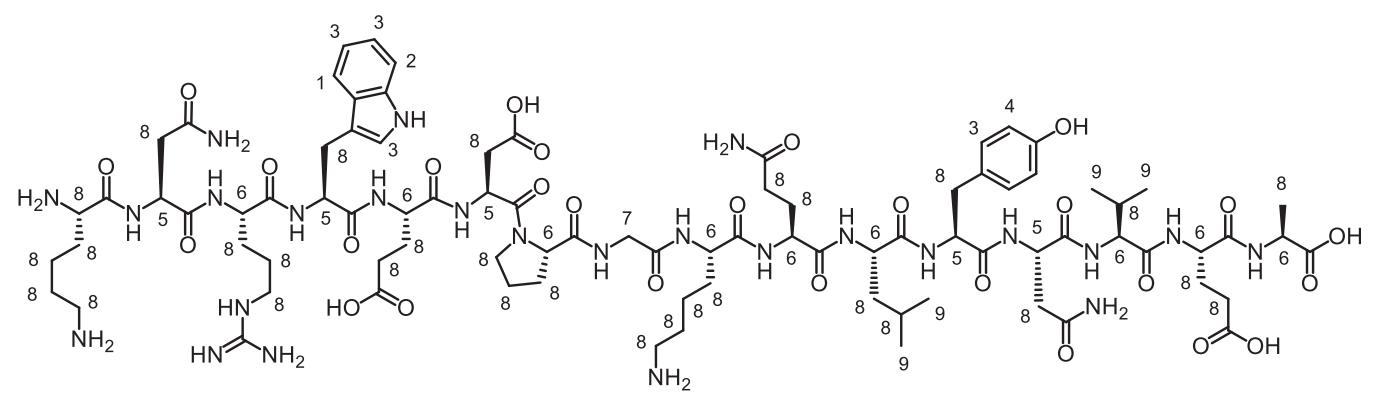

6.11

Figure 6.3. The 16-aa peptide 6.11 and proton numbering for NMR signal assignment 


\subsection{Conclusion}

In conclusion, we have successfully developed a new method for peptide purification. The method used a concept that is completely different from any previously reported peptide purification techniques. It is easy to use and readily scalable, and gives peptides with good purity and recovery yields. Adapting the method for purification of several peptide drugs such as Bivalirudin (20 aa),[27] Ziconotide (25 aa),[28] Thymalfasin (28 aa),[29] Enfuvirtide (36 aa)[30] and Pramlintide (39 aa)[31] is underway.

\section{Acknowledgments}

Financial support from US NSF (CHE-0647129 and CHE-1111192), MTU Biotech Research Center, MUCI and MTU REF Technology Commercialization; the assistance from Mr. Dean W. Seppala (electronics) and Mr. Jerry L. Lutz (NMR); and an NSF equipment grant (CHE-9512445 and CH-1048655) are gratefully acknowledged. 


\section{References and Notes}

1. Bellmann-Sickert, K. and A.G. Beck-Sickinger, Peptide drugs to target $G$ protein-coupled receptors. Trends in Pharmacological Sciences, 2010, 31(9): p. $434-441$.

2. Vlieghe, P., V. Lisowski, J. Martinez, et al., Synthetic therapeutic peptides: science and market. Drug Discovery Today, 2010, 15(1-2): p. 40-56.

3. Bray, B.L., Large-scale manufacture of peptide therapeutics by chemical synthesis. Nature Reviews Drug Discovery, 2003, 2(7): p. 587-593.

4. Akcay, G. and K. Kumar, A new paradigm for protein design and biological self-assembly. Journal of Fluorine Chemistry, 2009, 130(12): p. 1178-1182.

5. Montanari, V. and K. Kumar, Enabling routine fluorous capping in solid phase peptide synthesis. Journal of Fluorine Chemistry, 2006, 127(4-5): p. $565-570$.

6. Filippov, D.V., D.J. van Zoelen, S.P. Oldfield, et al., Use of benzyloxycarbonyl (Z)-based fluorophilic tagging reagents in the purification of synthetic peptides. Tetrahedron Letters, 2002, 43(43): p. 7809-7812.

7. Fustero, S., A.G. Sancho, G. Chiva, et al., Fluorous (trimethylsilyl)ethanol: A new reagent for carboxylic acid tagging and protection in peptide synthesis. Journal of Organic Chemistry, 2006, 71(8): p. 3299-3302.

8. de Visser, P.C., M. van Helden, D.V. Filippov, et al., A novel, base-labile fluorous amine protecting group: synthesis and use as a tag in the purification of synthetic peptides. Tetrahedron Letters, 2003, 44(50): p. 9013-9016. 
9. Montanari, V. and K. Kumar, A fluorous capping strategy for Fmoc-based automated and manual solid-phase peptide synthesis. European Journal of Organic Chemistry, 2006(4): p. 874-877.

10. Montanari, V. and K. Kumar, Just add water: A new fluorous capping reagent for facile purification of peptides synthesized on the solid phase. Journal of the American Chemical Society, 2004, 126(31): p. 9528-9529.

11. Bang, D. and S.B.H. Kent, HiS(6) tag-assisted chemical protein synthesis. Proceedings of the National Academy of Sciences of the United States of America, 2005, 102(14): p. 5014-5019.

12. Shogren-Knaak, M.A. and B. Imperiali, A reversible affinity tag for the purification of N-glycolyl capped peptides. Tetrahedron Letters, 1998, 39(45): p. $8241-8244$.

13. Funakoshi, S., H. Fukuda, and N. Fujii, Chemmoselective one-step purification method for peptide synthesized by the solid-phase technique. Proceedings of the National Academy of Sciences of the United States of America, 1991, 88(16): p. 6981-6985.

14. Vizzavona, J., M. Villain, and K. Rose, Covalent capture purification of polypeptides after SPPS via a linker removable under very mild conditions. Tetrahedron Letters, 2002, 43(48): p. 8693-8696.

15. Canne, L.E., R.L. Winston, and S.B.H. Kent, Synthesis of a versatile purification handle for use with Boc chemistry solid phase peptide synthesis. Tetrahedron Letters, 1997, 38(19): p. 3361-3364. 
16. Ball, H.L. and P. Mascagni, Chemical synthesis and purification of proteins: A methodology. International Journal of Peptide and Protein Research, 1996, 48(1): p. 31-47.

17. Fang, S. and S. Fueangfung, Scalable Synthetic Oligodeoxynucleotide Purification with Use of a Catching by Polymerization, Washing, and Releasing Approach. Organic Letters, 2010, 12(16): p. 3720-3723.

18. Yuan, Y., S. Fueangfung, X. Lin, et al., Synthetic 5 '-phosphorylated oligodeoxynucleotide purification through catching full-length sequences by polymerization. Rsc Advances, 2012, 2(7): p. 2803-2808.

19. Fang, S., S. Fueangfung, X. Lin, et al., Synthetic oligodeoxynucleotide purification by polymerization of failure sequences. Chemical Communications, 2011, 47(4): p. 1345-1347.

20. Athanassopoulos, P., K. Barlos, D. Gatos, et al., Application of 2-chlorotrityl chloride in convergent peptide-synthesis. Tetrahedron Letters, 1995, 36(31): p. $5645-5648$.

21. Barlos, K., D. Gatos, S. Kapolos, et al., Application of 2-chlorotrityl resin in solid-phase synthesis of (Leu(15)-gastrin-I and unsulfated cholecystokinin octapeptide-selective o-deprotection of tyrosine. International Journal of Peptide and Protein Research, 1991, 38(6): p. 555-561.

22. Harre, M., K. Nickisch, and U. Tilstam, An efficient method for activation and recycling of trityl resins. Reactive \& Functional Polymers, 1999, 41(1-3): p. $111-114$. 
23. Barlos, K., D. Gatos, S. Kapolos, et al., Esterification of partially protected peptide-fragments with resins-utilization of 2-chlorotritylchloride for synthesis of leu-15-gastrin-I. Tetrahedron Letters, 1989, 30(30): p. 3947-3950.

24. Naganawa, A., T. Matsui, M. Ima, et al., Further optimization of sulfonamide analogs as EP1 receptor antagonists: Synthesis and evaluation of bioisosteres for the carboxylic acid group. Bioorganic \& Medicinal Chemistry, 2006, 14(21): p. 7121-7137.

25. Tang, W. and S. Fang, Mono-acylation of symmetric diamines in the presence of water. Tetrahedron Letters, 2008, 49(41): p. 6003-6006.

26. Frade, R., J. Hermann, and M. Barel, A 16 amino-acid synthetic peptide derived from human $C 3 D$ triggers proliferation and specific tyrosine phosphorylation of transformed CR-2-positive human-lymphocytes and of normal resting lymphocytes-B. Biochemical and Biophysical Research Communications, 1992, 188(2): p. 833-842.

27. Garner, W.L., J.A. Linden, and G.S. Chrysant, The clinical utility of bivalirudin in patients with coronary artery disease. Cardiovascular \& hematological agents in medicinal chemistry, 2013, 11(1): p. 44-8.

28. Pope, J.E. and T.R. Deer, Ziconotide: a clinical update and pharmacologic review. Expert Opinion on Pharmacotherapy, 2013, 14(7): p. 957-966.

29. Ciancio, A. and M. Rizzetto, Thymalfasin in the treatment of hepatitis $B$ and C, in Thymosins in Health and Disease, E. Garaci and A.L. Goldstein, Editors. 2010. p. 141-146. 
30. Joly, V., K. Jidar, M. Tatay, et al., Enfuvirtide: from basic investigations to current clinical use. Expert Opinion on Pharmacotherapy, 2010, 11(16): p. 2701-2713.

31. Younk, L.M., M. Mikeladze, and S.N. Davis, Pramlintide and the treatment of diabetes: a review of the data since its introduction. Expert Opinion on Pharmacotherapy, 2011, 12(9): p. 1439-1451. 


\section{Chapter 7}

\section{Future Research Plan}

Synthetic ODNs and peptides have wide applications in therapeutics, genomics and forensic science. In the previous chapters, we described the novel technologies for ODN and peptide purification. We used catching by polymerization approach to purify these biooligomers. The result of the technology is satisfactory. In future, our efforts may be directed to extend the catching failure sequences by polymerization approach to peptide purification, using the catching-full length sequence by polymerization approach to purify peptides of much longer sequences and specific drugs at large scales, using the catching by polymerization methods to purify RNA, phosphorothioates, and other modified nucleic acids, and adopt the technologies for high throughput purification of ODNs.

\subsection{For Peptide Purification}

\subsubsection{Catching Failure Sequences by Polymerization}

In chapter 6 , we discussed the purification of peptide by polymerizing fulllength sequences. Despite the satisfactory result, the peptide must be cleaved from the gel during purification. We proposed a purification method by catching failure sequences which does not need a cleavage step. Failure sequences are capped right after each synthetic cycle by suitable reagents containing polymerizable group. We designed two capping reagents; the first one is methacrylic anhydride 7.1. The second reagent is the pentafluro compound 7.2. 


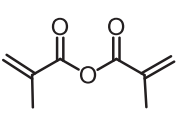

7.1

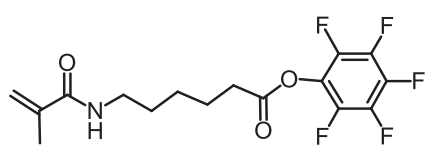

7.2

Figure 7.1. Proposed capping reagents

The failure sequences will be capped with 7.1 or 7.2 during automated synthesis. After cleavage, they will be polymerized leaving full-length peptide in solution, which will be extracted with suitable solvents. Small molecules will be removed by precipitation of the full-length sequences from a TFA solution with cold ether. The peptides, which normally carry positive charges under these conditions, are not soluble in ether, while small molecules, which are neutral, are soluble in ether.

\subsubsection{Purification of Longer Sequences and at Large Scale}

We purified peptides that contain 6 and 16 amino acids by the catching fulllength sequence by polymerization approach. In this method, a polymerizable tag is attached only once at the end of the amino terminus so it is suitable for longer peptide purification. We will use the method to purify peptides that have more than 30 amino acids. We will also use the technique to purify specific peptide durgs such as Bivalirudin,[1] Ziconotide,[2] Thymalfasin,[3] Enfuvirtide,[4] and Pramlintide[5]. Further, we will use the method to purify at scales that can afford gram quantities of pure peptides. 


\subsection{For ODN and RNA Purification}

\subsubsection{RNA Purification}

Purification of oligoribonucleotides is also highly important because of their wide applications in medicine and biology. RNA purification is more challenging due to the ubiquitous enzyme known as RNAse. We propose the purification of oligoribonucleotide using the catching by polymerization ODN purification technologies. Both technologies of ODN purification can be used to purify oligoribonucleotide by slight modification of the current methods.

\subsubsection{Longer Sequence Purification, Large Scale Purification and High Throughput Purification}

In chapter 4, we discussed the purification of a 151 mer ODN by catching

full-length sequence polymerization. We propose to purify even longer ODNs such as those that are as long as 200 mer. This method is suitable to purify longer ODNs because tagging with an expensive polymerizable phosphoramidite is only required at the end of synthesis. Because of the high demand for pure ODN in industrial scales, we propose to test the suitability of the technology to purify ODNs at gram scales. 


\subsubsection{Purification of Phosphorothioates}

Phosphorothioates have increasing applications in therapeutics. In phosphorothioates 7.4, the oxygen atom in the phosphate backbone is replaced with a sulfur atom. This structure change makes the ODN more resistant to enzymatic hydrolysis (Figure 7.2).[6] The delivery of this modified ODN to the interior of cell is more efficient than normal ODN. Because of the applications of phosphorathioates, we propose to purify phosphorothioates by the catching polymerization approach.

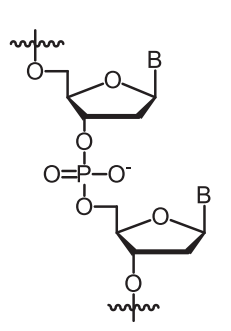

7.3

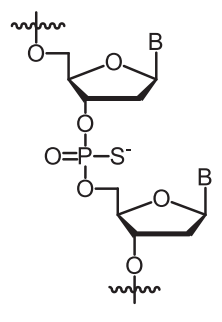

7.4

Figure 7.2. Phosphodiester and phosphorothioate linkages 


\section{References and Notes}

1. Warkentin, T.E., A. Greinacher, and A. Koster, Bivalirudin. Thrombosis and Haemostasis, 2008, 99(5): p. 830-839.

2. Miljanich, G., Ziconotide: neuronal calcium channel blocker for treating severe chronic pain. Current Medicinal Chemistry, 2004, 11(23): p. 30293040 .

3. Ciancio, A. and M. Rizzetto, Thymalfasin in the treatment of hepatitis $B$ and C. Annals of the New York Academy of Sciences, 2010, 1194(1): p. 141-146.

4. Lazzarin, A., B. Clotet, D. Cooper, et al., Efficacy of enfuvirtide in patients infected with drug-resistant HIV-1 in Europe and Australia. New England Journal of Medicine, 2003, 348(22): p. 2186-2195.

5. Younk, L.M., M. Mikeladze, and S.N. Davis, Pramlintide and the treatment of diabetes: a review of the data since its introduction. Expert Opinion on Pharmacotherapy, 2011, 12(9): p. 1439-1451.

6. Skerra, A., Phosphorothioate primers improve the amplification of DNA sequences by DNA polymerases with proofreading activity. Nucleic Acids Research, 1992, 20(14): p. 3551-3554. 


\section{Appendix A}

Supporting Information for Chapter 2

Synthetic Oligodeoxynucleotide Purification by Capping Failure Sequences with a Methacrylamide Phosphoramidite Followed by Polymerization 


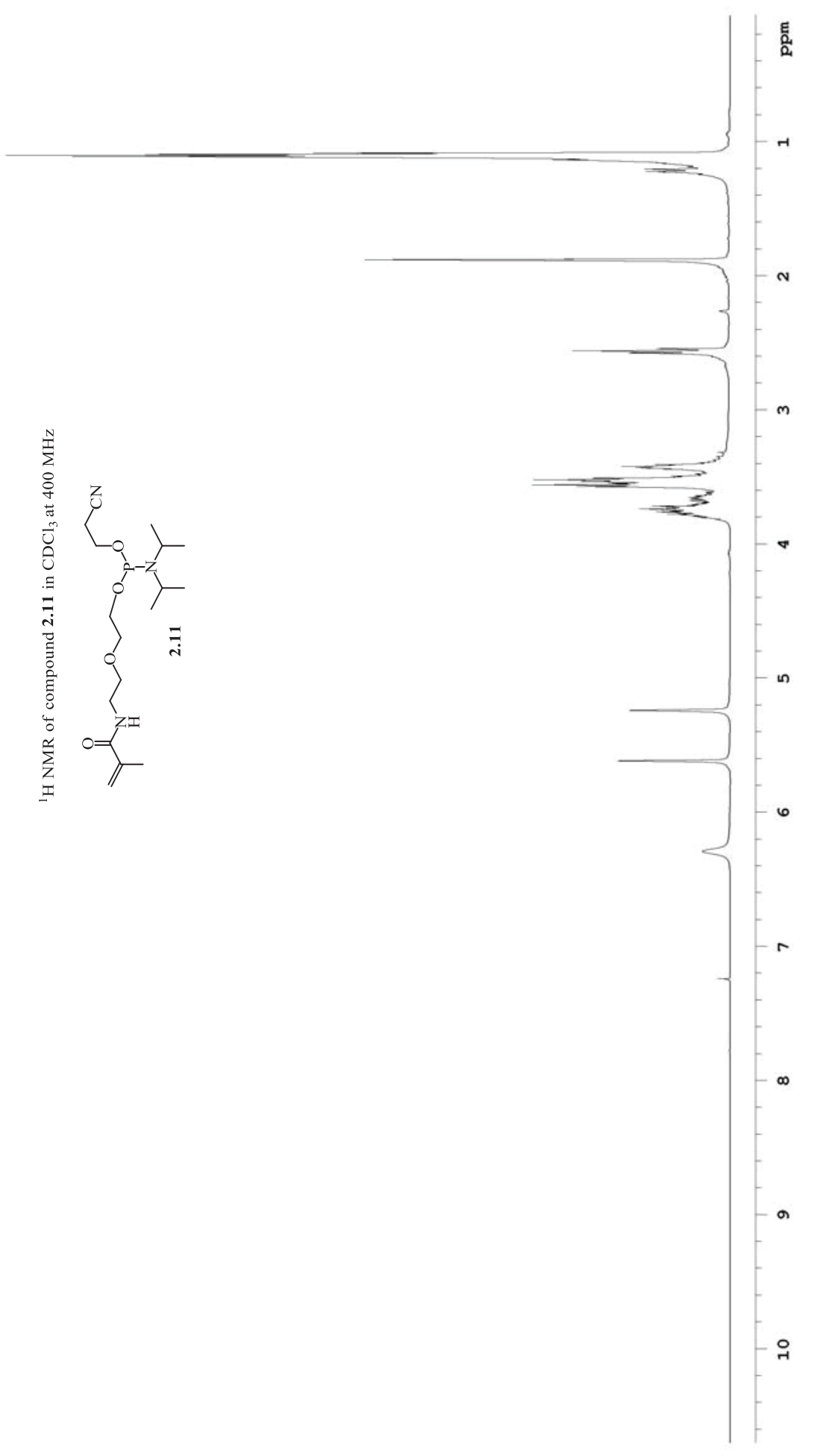

Figure A.1. ${ }^{1} \mathrm{H}$ NMR of compound 2.11 


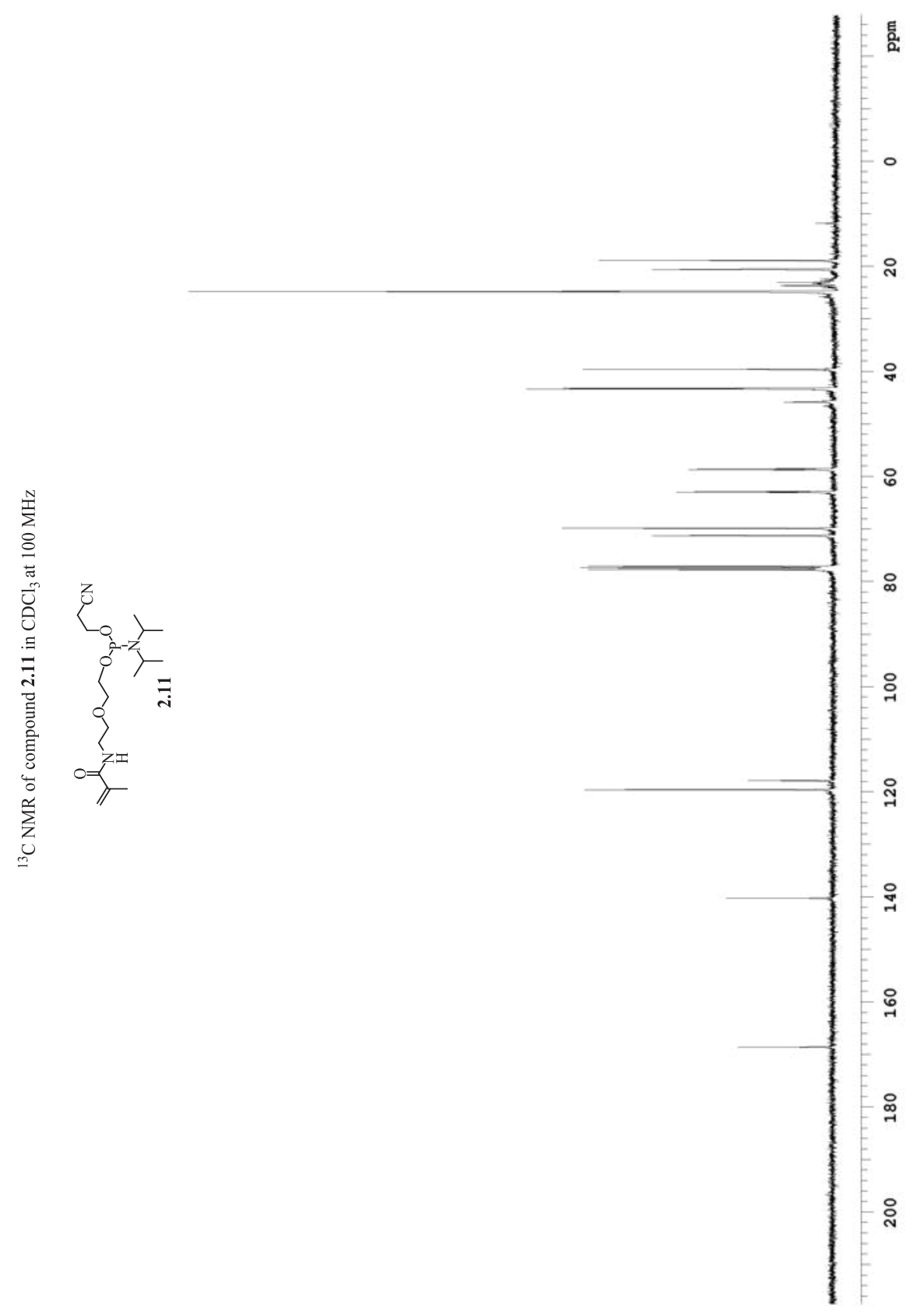

Figure A.2. ${ }^{13} \mathrm{C}$ NMR of compound 2.11 


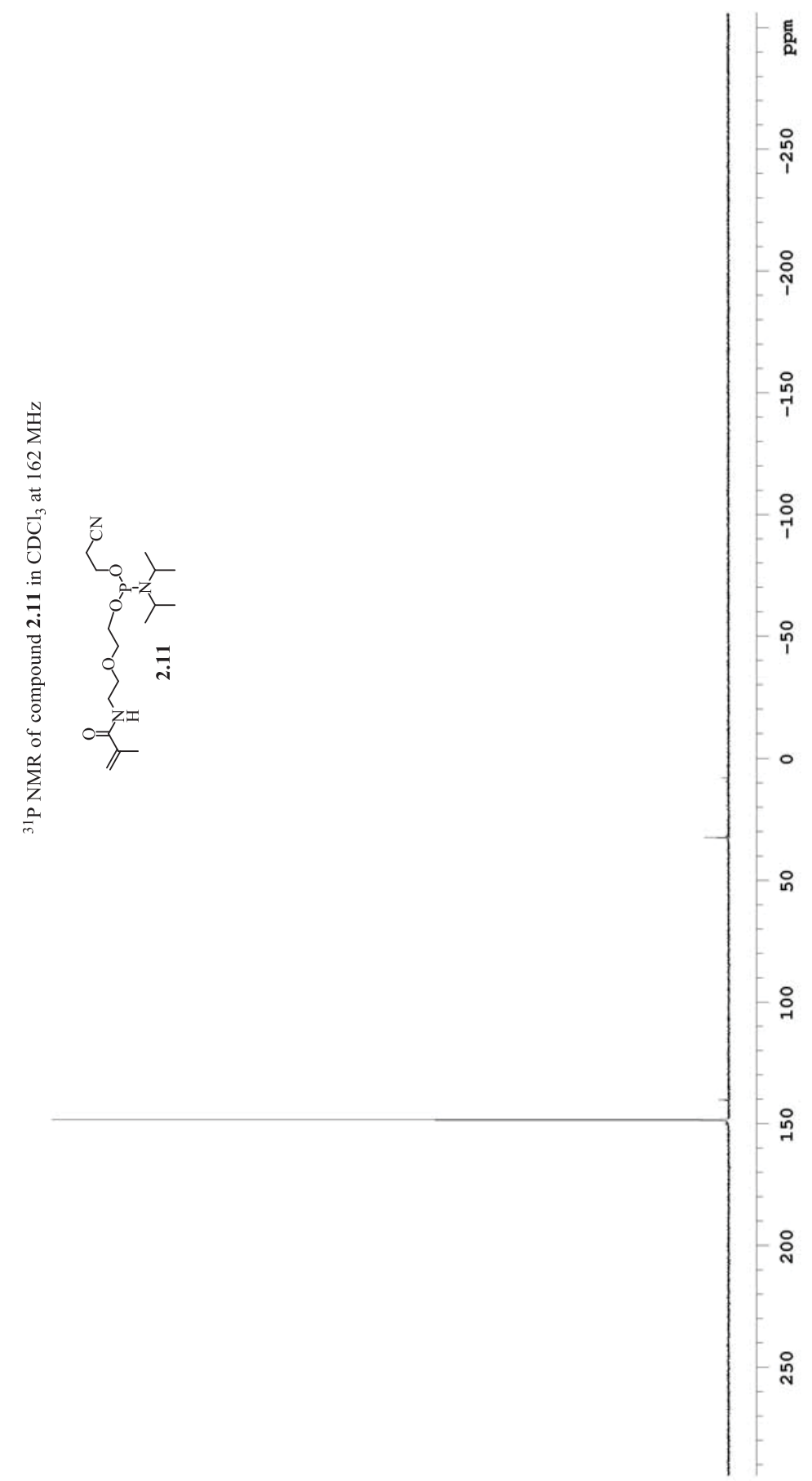

Figure A.3. ${ }^{31} \mathrm{P}$ NMR of compound 2.11 


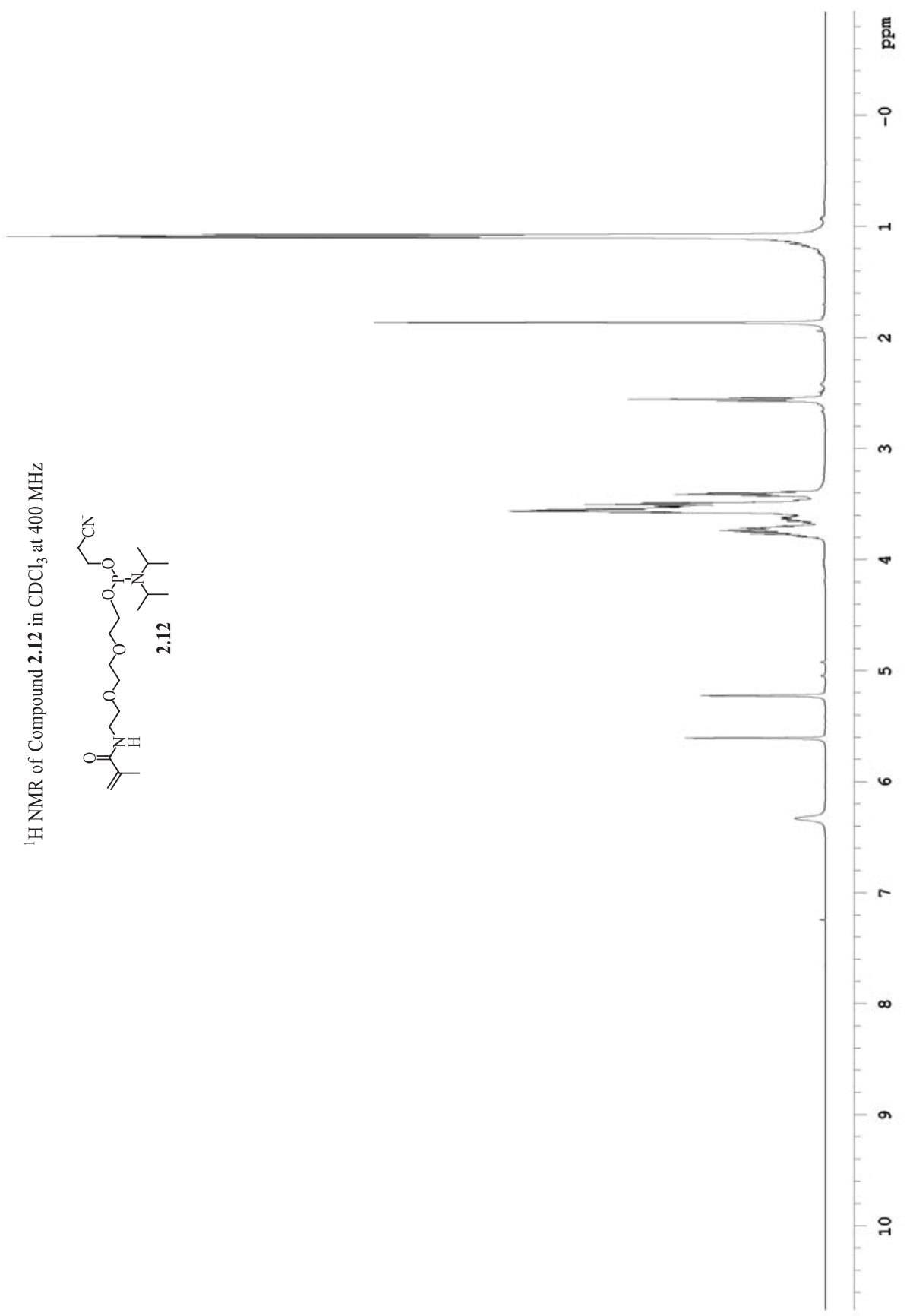

Figure A.4. ${ }^{1} \mathrm{H}$ NMR of compound 2.12 


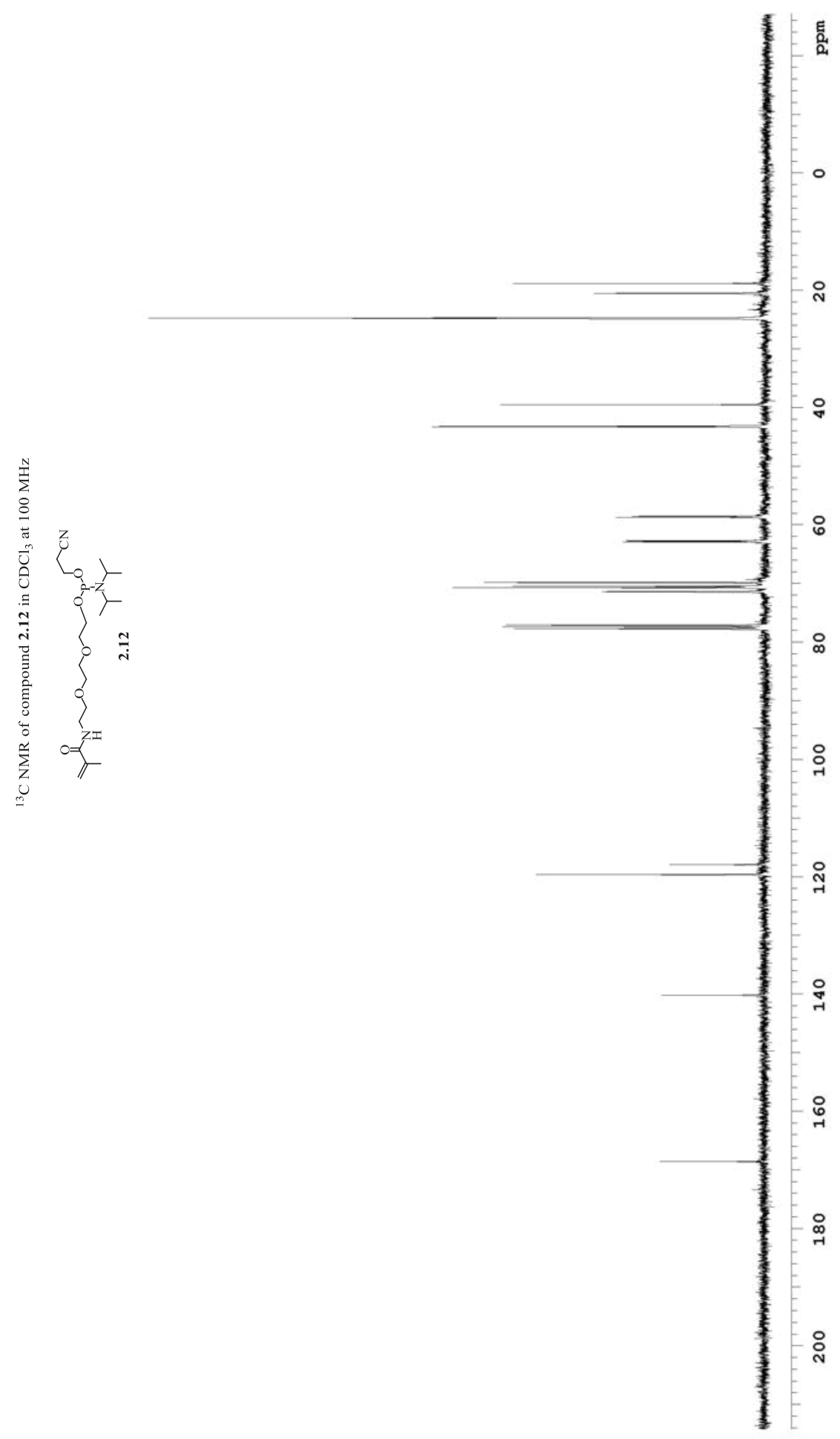

Figure A.5. ${ }^{13} \mathrm{C}$ NMR of compound 2.12 179 


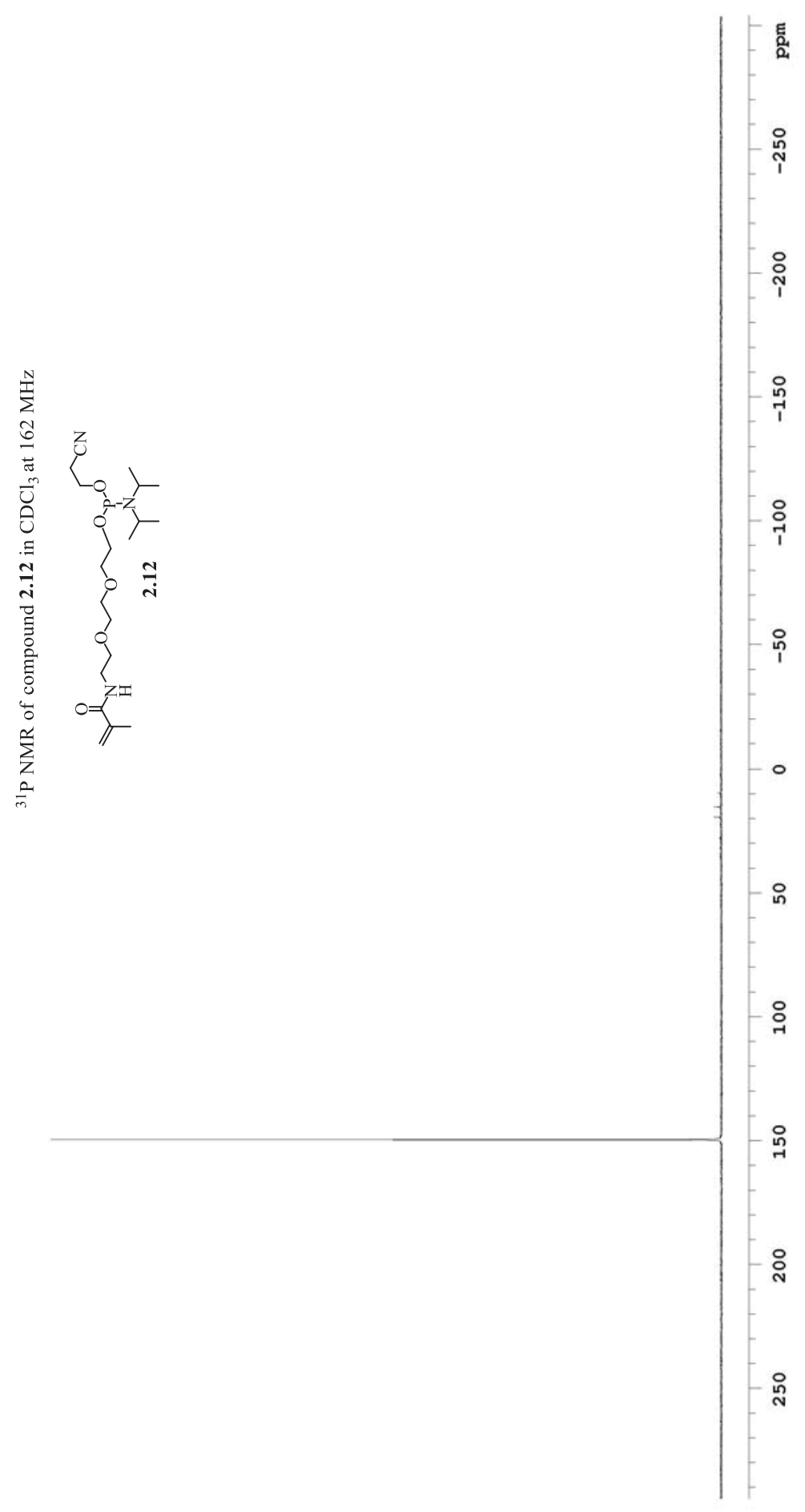

Figure A.6. ${ }^{31} \mathrm{P}$ NMR of compound 2.12 


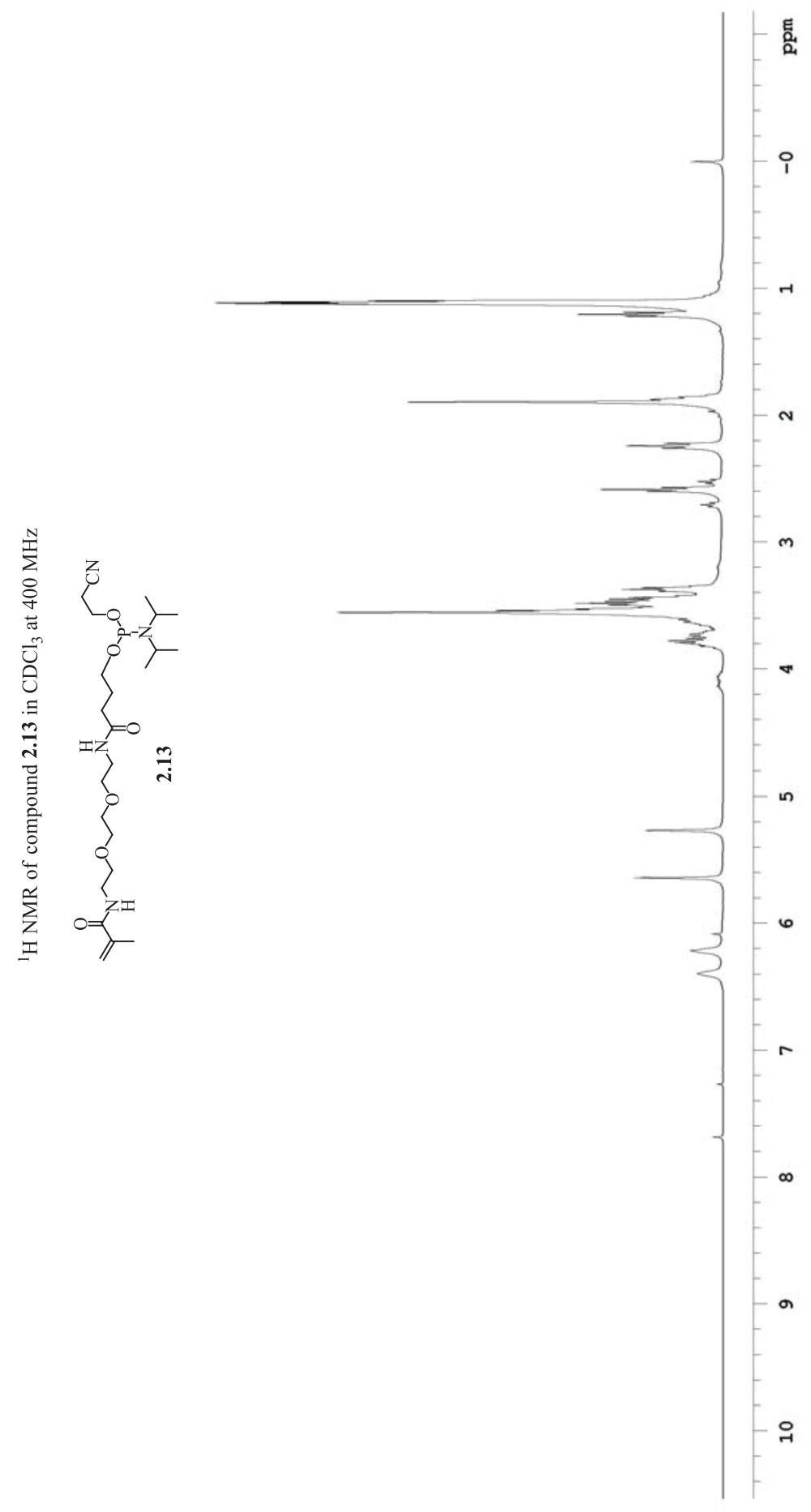

Figure A.7. ${ }^{1} \mathrm{H}$ NMR of compound 2.13 


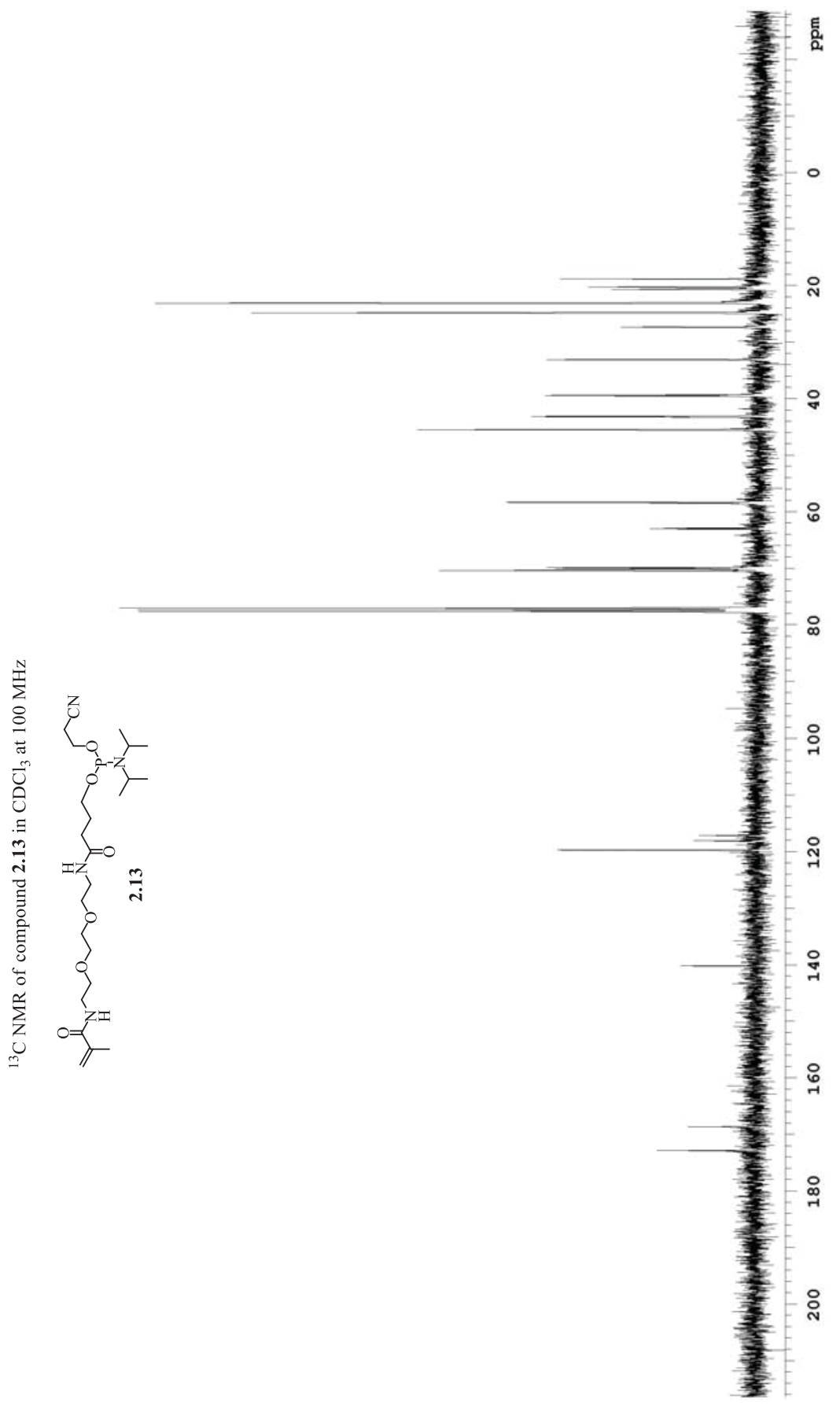

Figure A.8. ${ }^{13} \mathrm{C}$ NMR of compound 2.13 


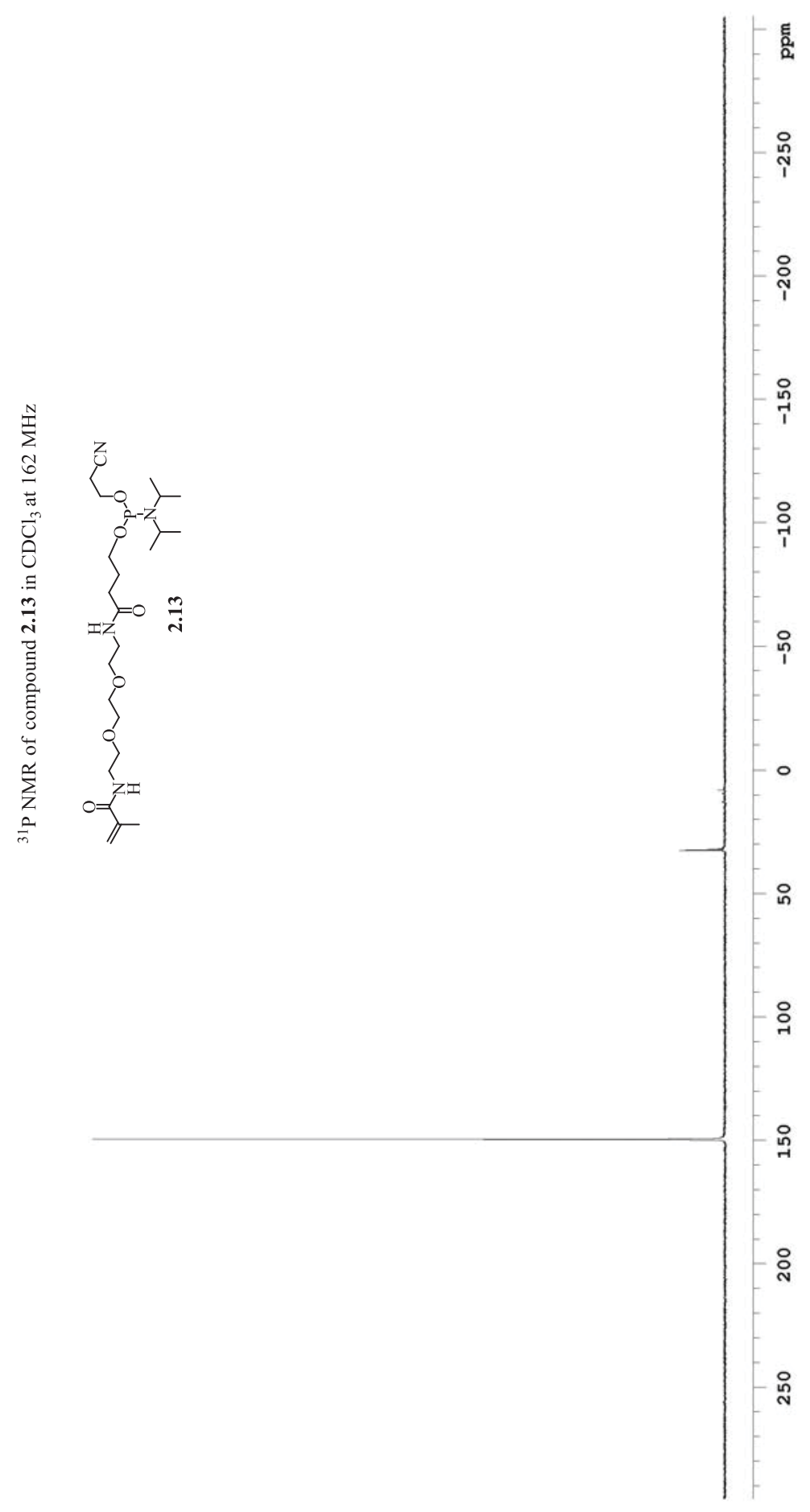

Figure A.9. ${ }^{13} \mathrm{P}$ NMR of compound 2.13 


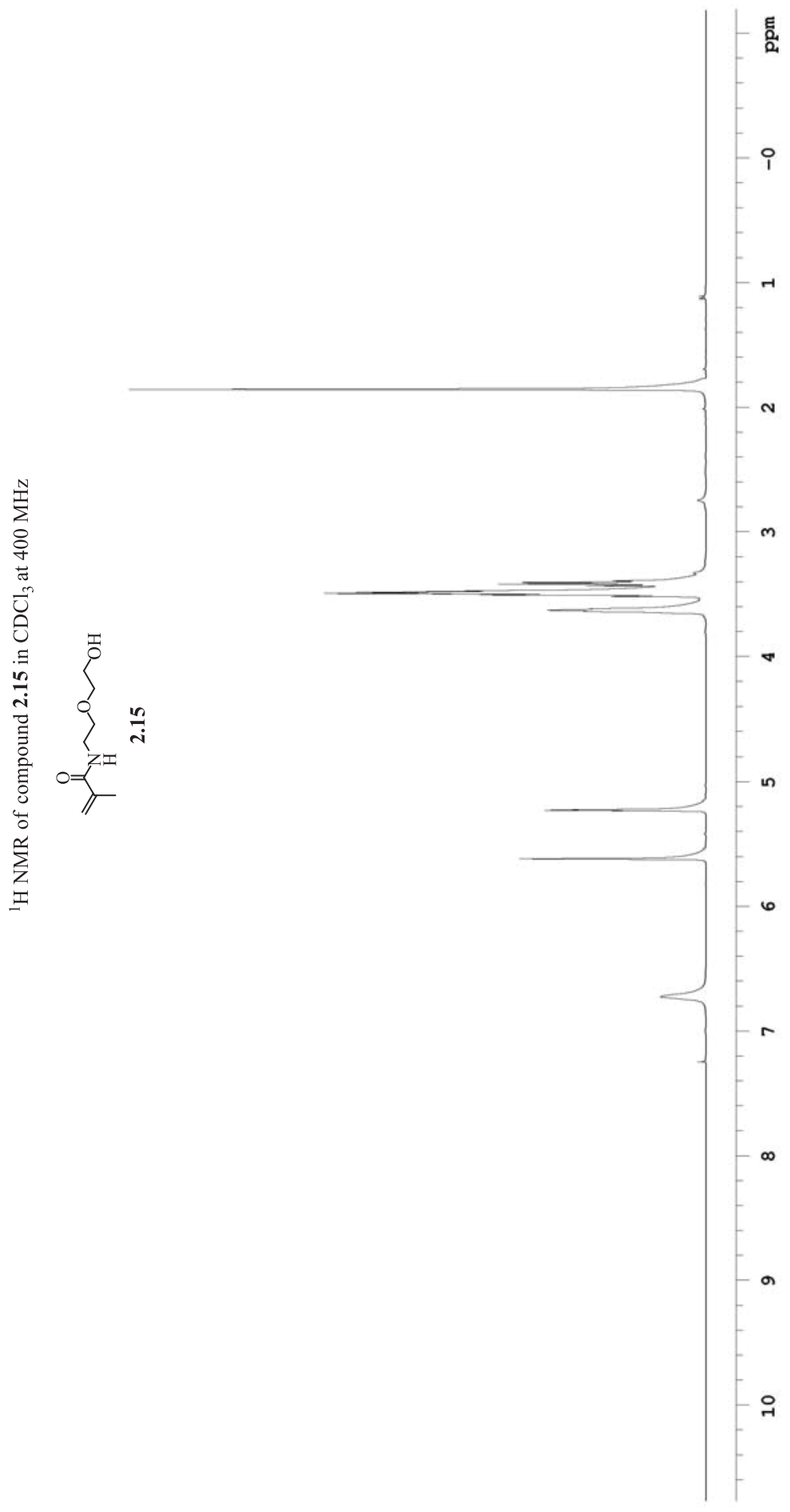

Figure A.10. ${ }^{1} \mathrm{H}$ NMR of compound 2.15 

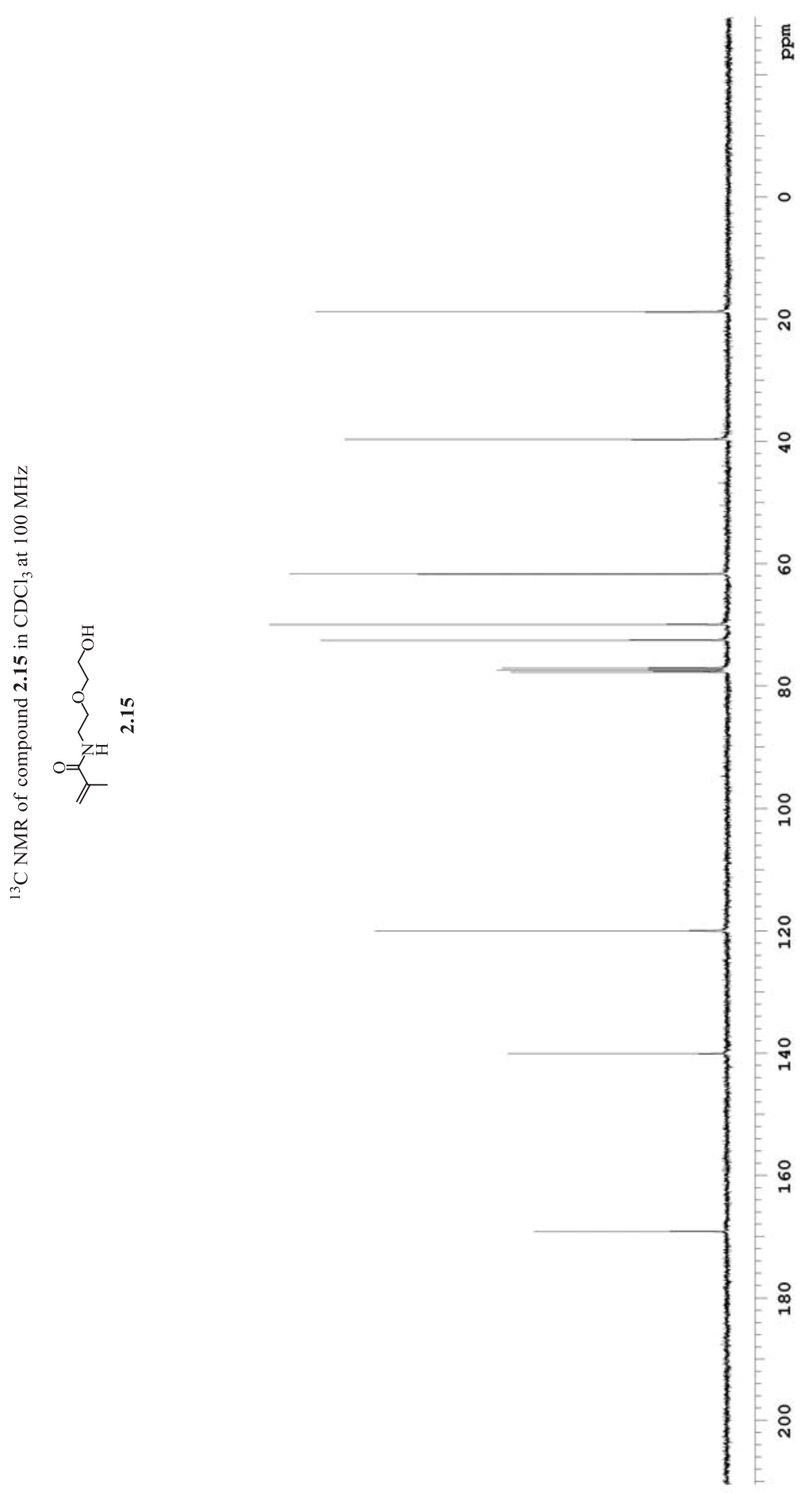

Figure A.11. ${ }^{13} \mathrm{C}$ NMR of compound 2.15 


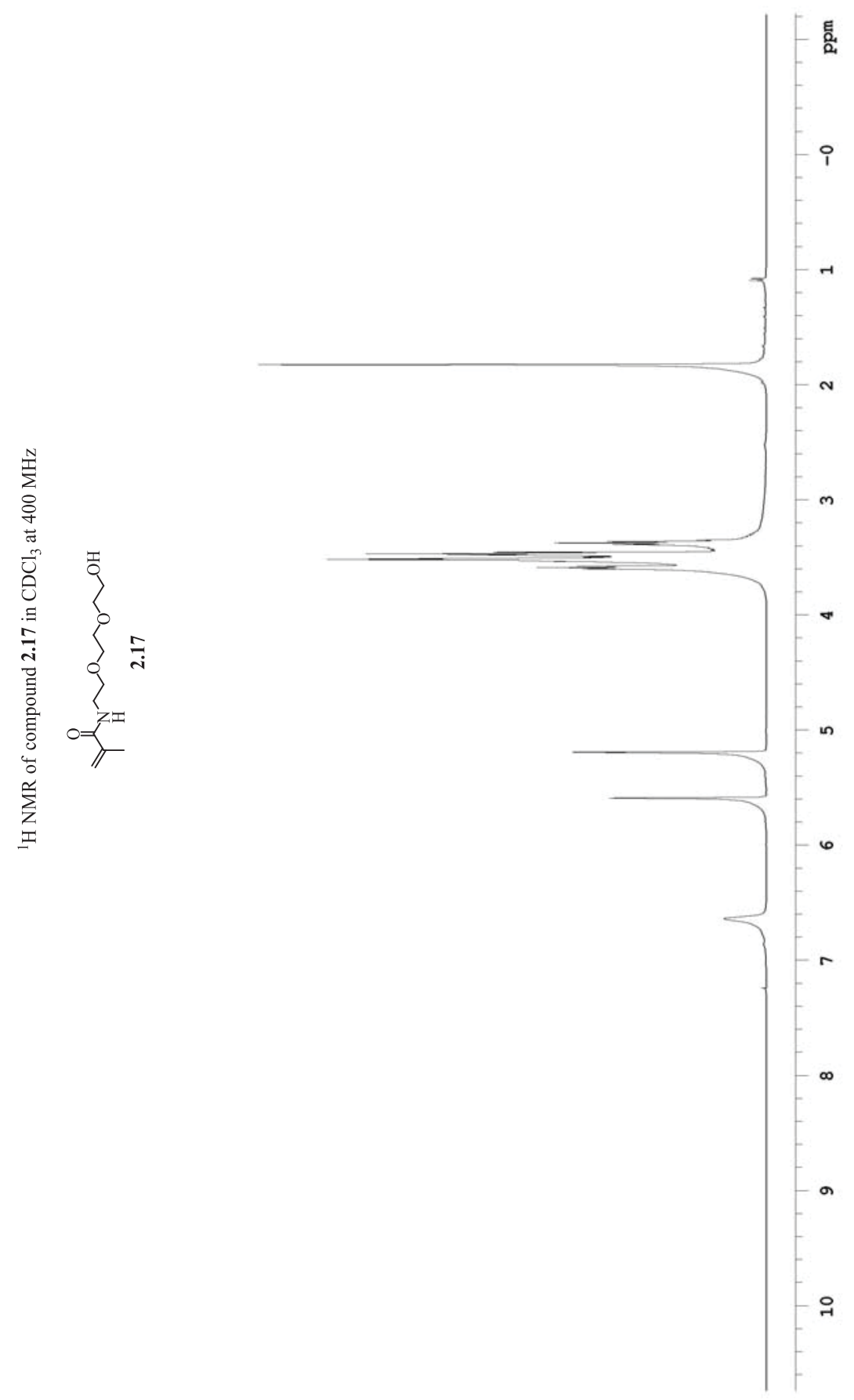

Figure A.12. ${ }^{1} \mathrm{H}$ NMR of compound 2.17 


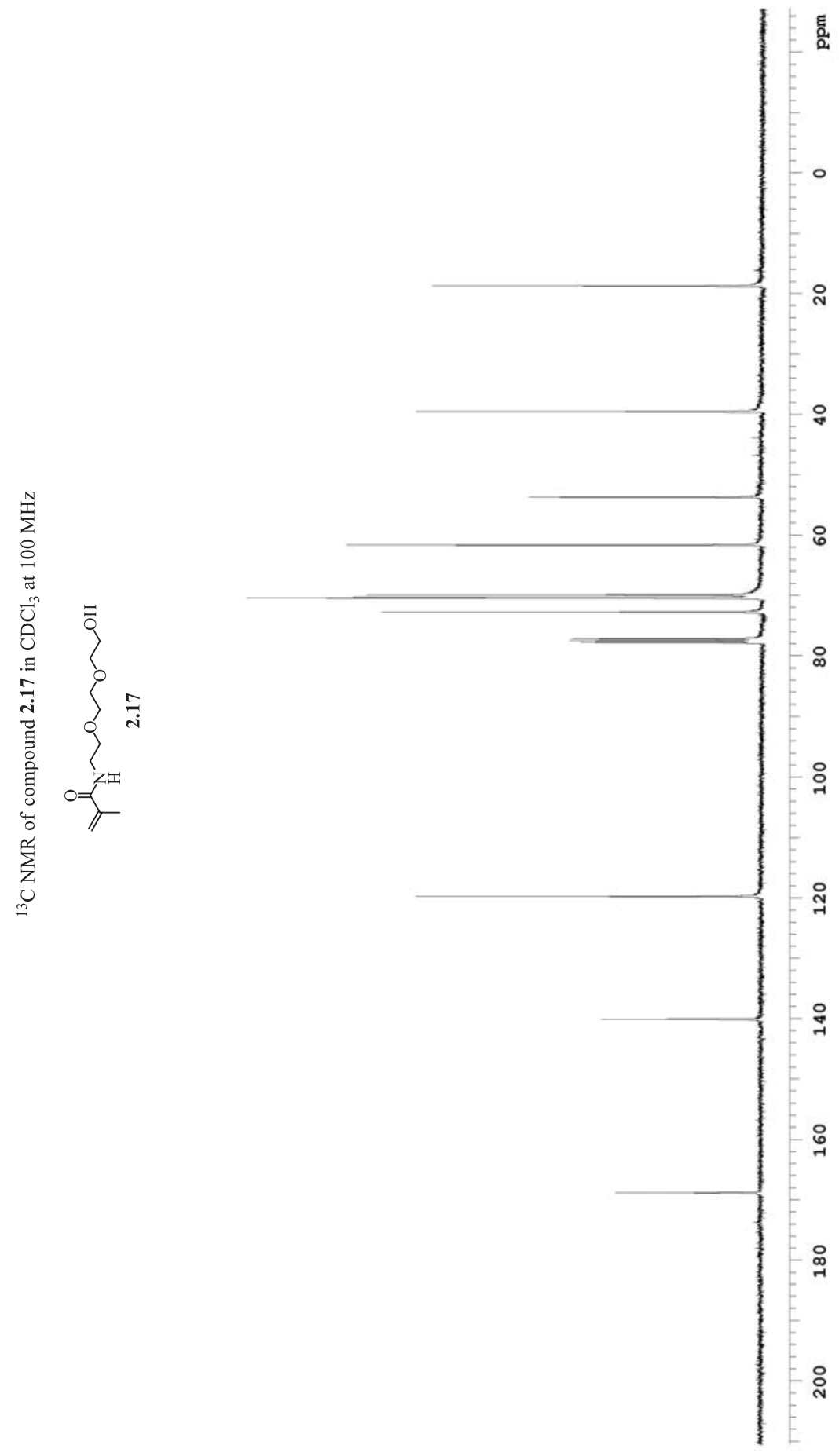

Figure A.13. ${ }^{13} \mathrm{C}$ NMR of compound 2.17 


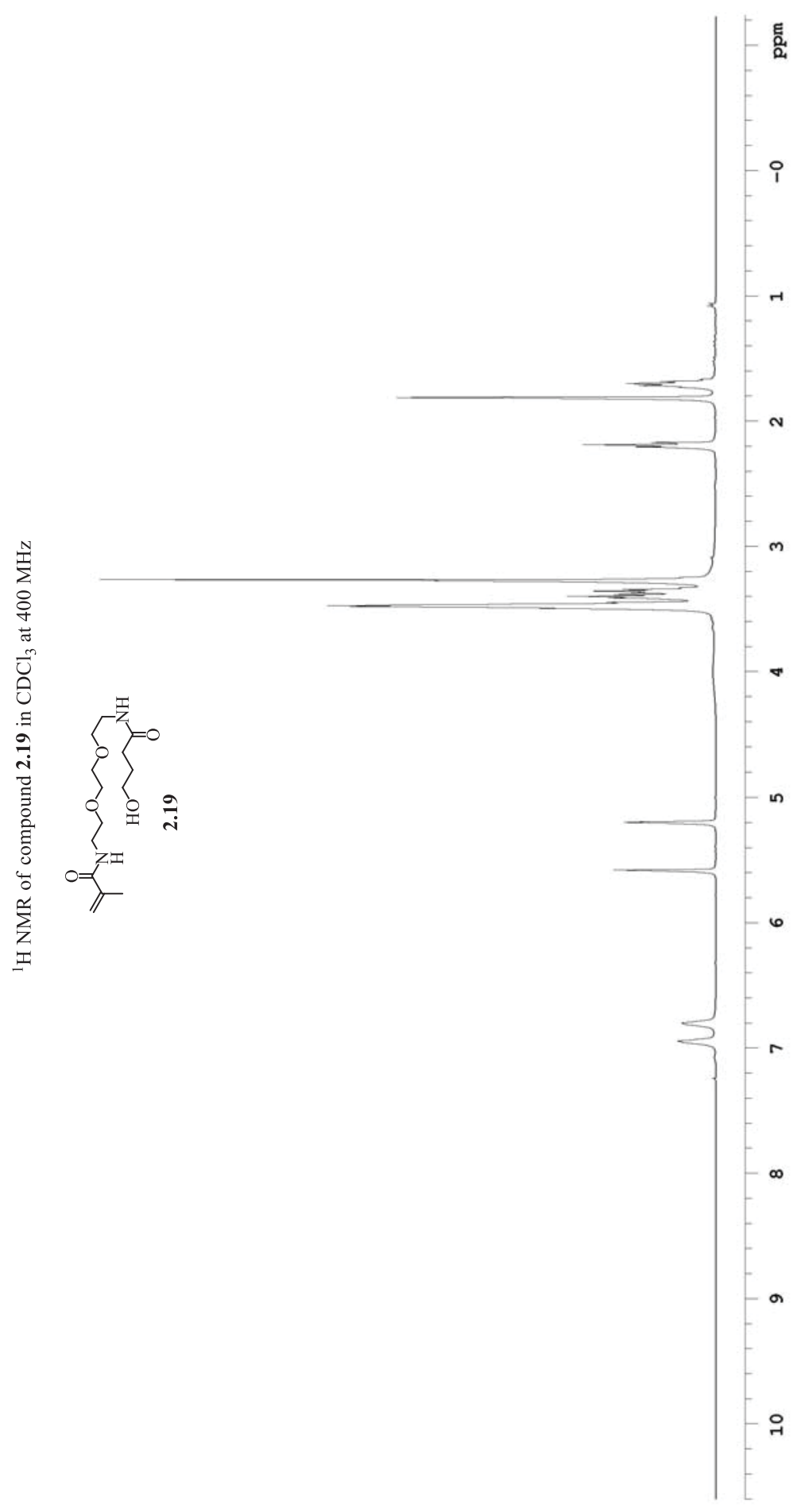

Figure A.14. ${ }^{1}$ H NMR of compound 2.19 


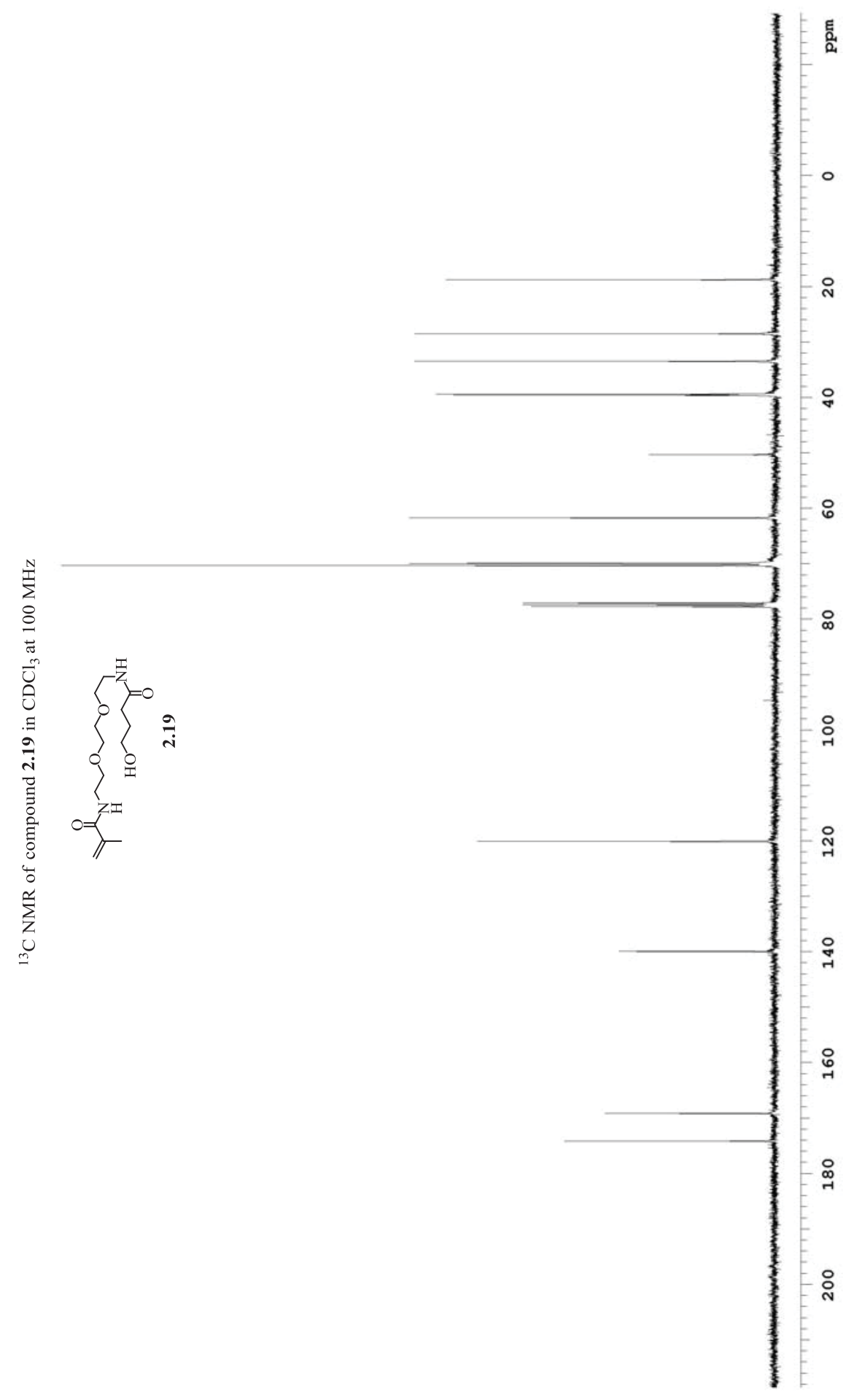

Figure A.15. ${ }^{13} \mathrm{C}$ NMR of compound 2.19 
Durga Pokharel DP-2059 ESI+
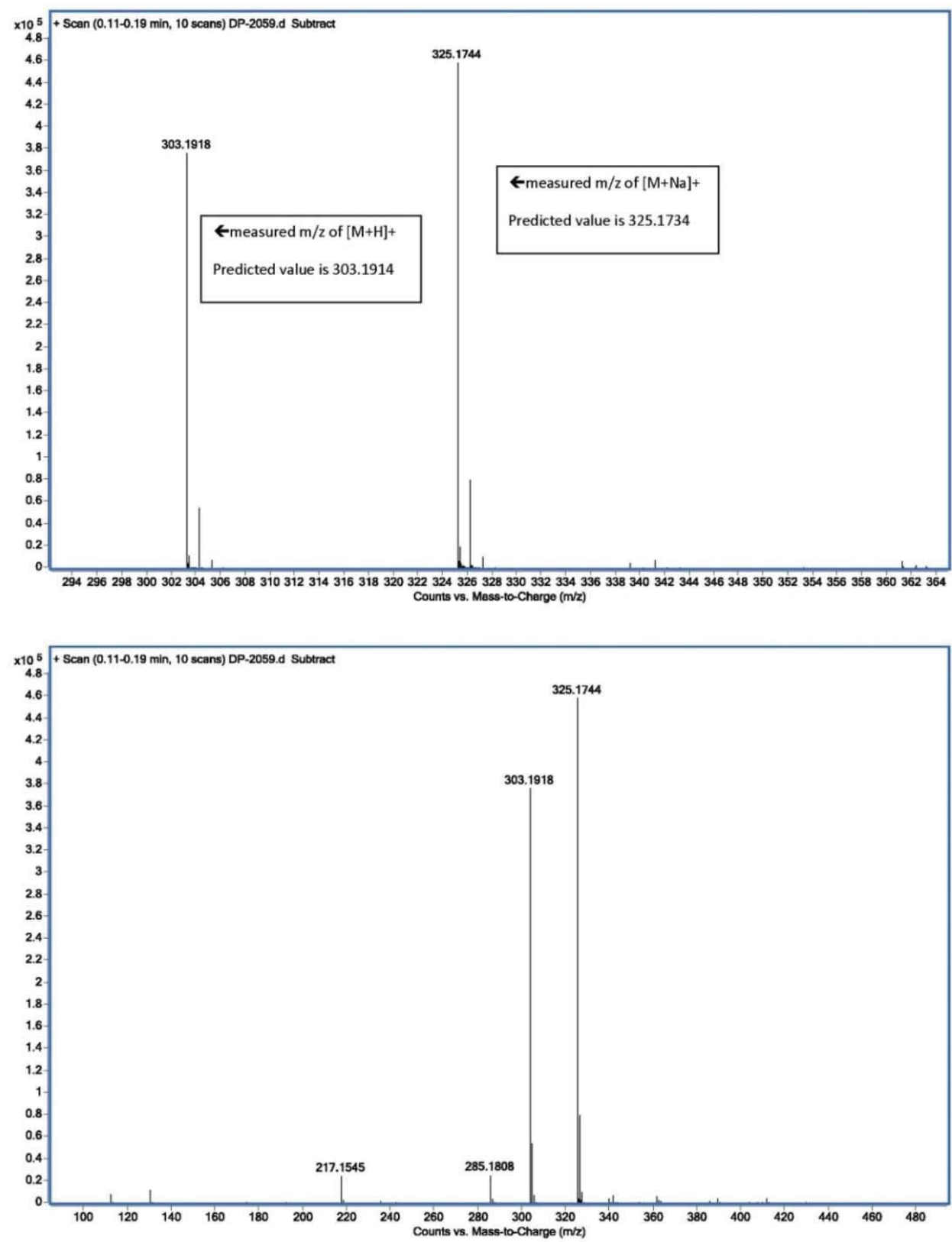

Figure A.16. ESI MS of compound 2.19 
Sample 2086

Negative ion ESI

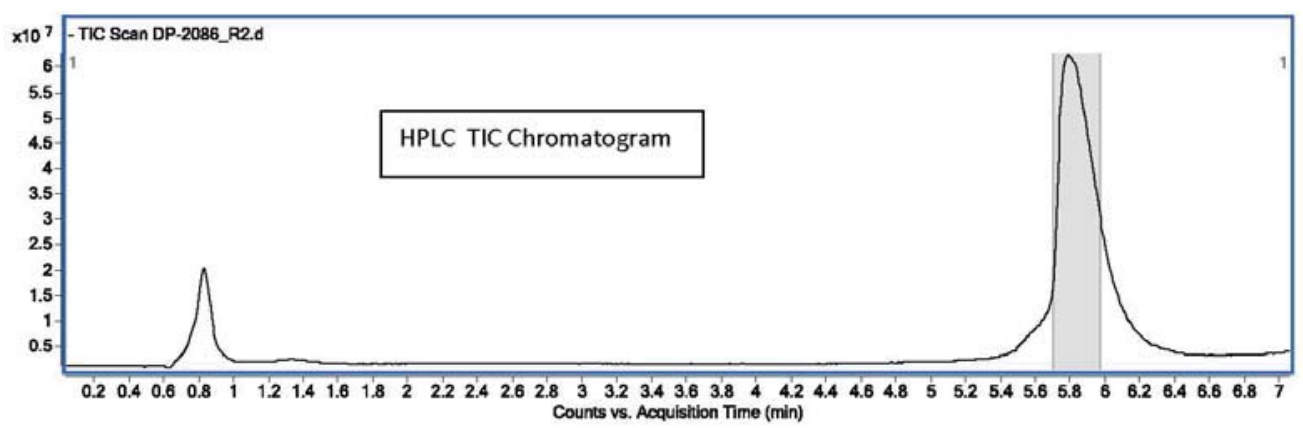

Raw data ESI- spectrum of highlighted area of the chromatogram:

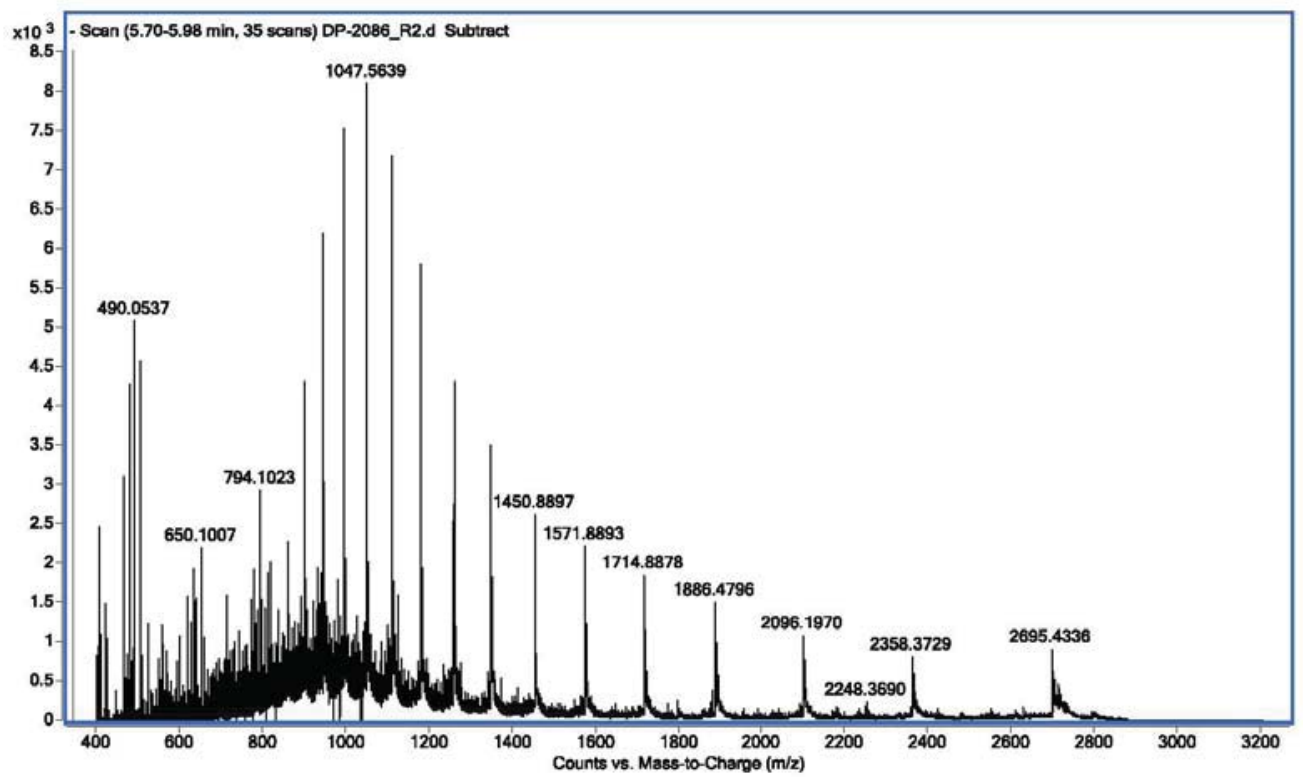

Figure A.17. LC MS of ODN 2.23 (continued next page) 
Maximum Entropy Deconvolution of the above spectrum:
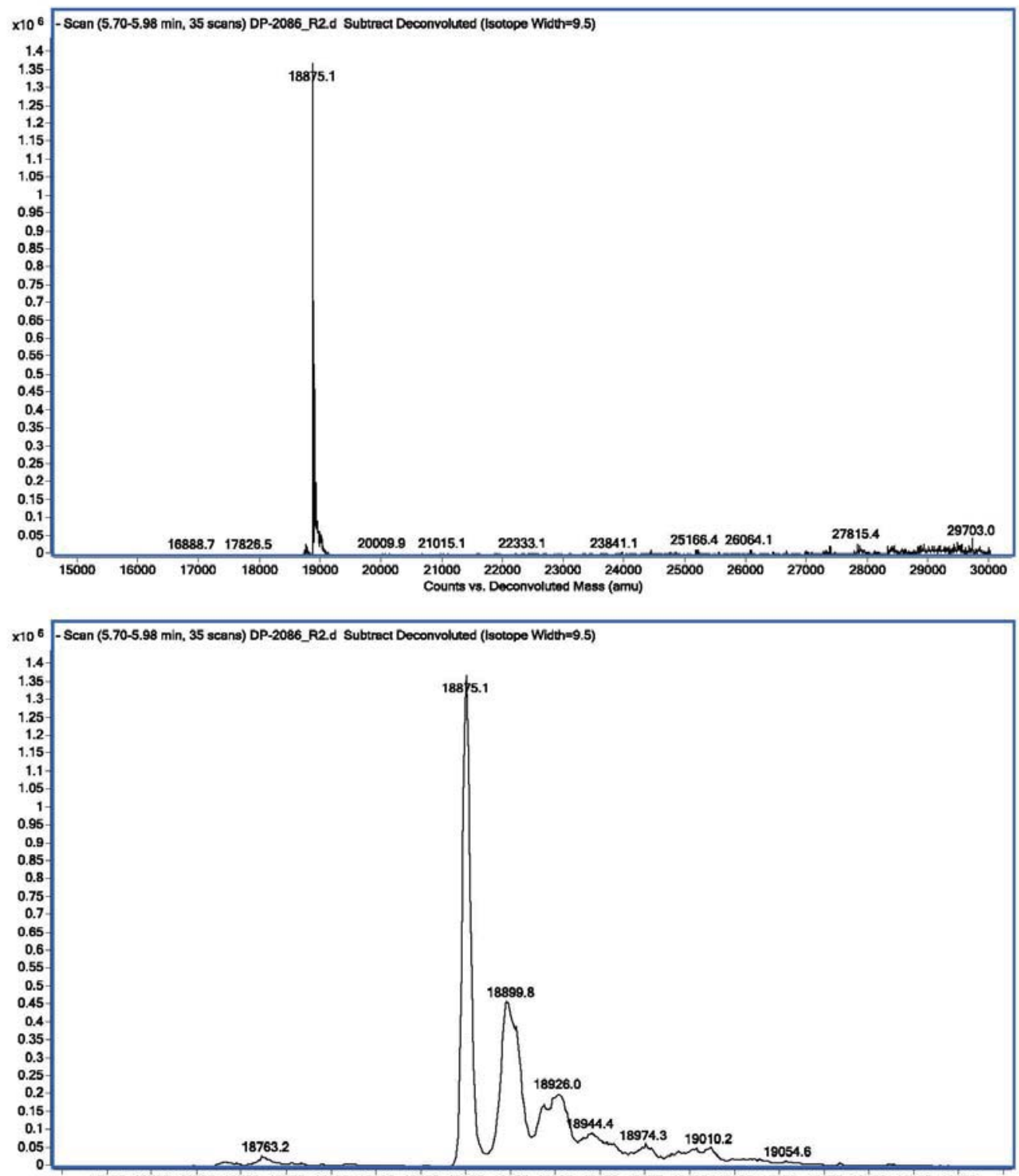

1867518700187251875018775188001882518850188751890018925189501897519000190251905019075191001912519150 Counts vs. Deconvoluted Mass (amu)

Figure A.17. LC MS of ODN 2.23 (continued) 


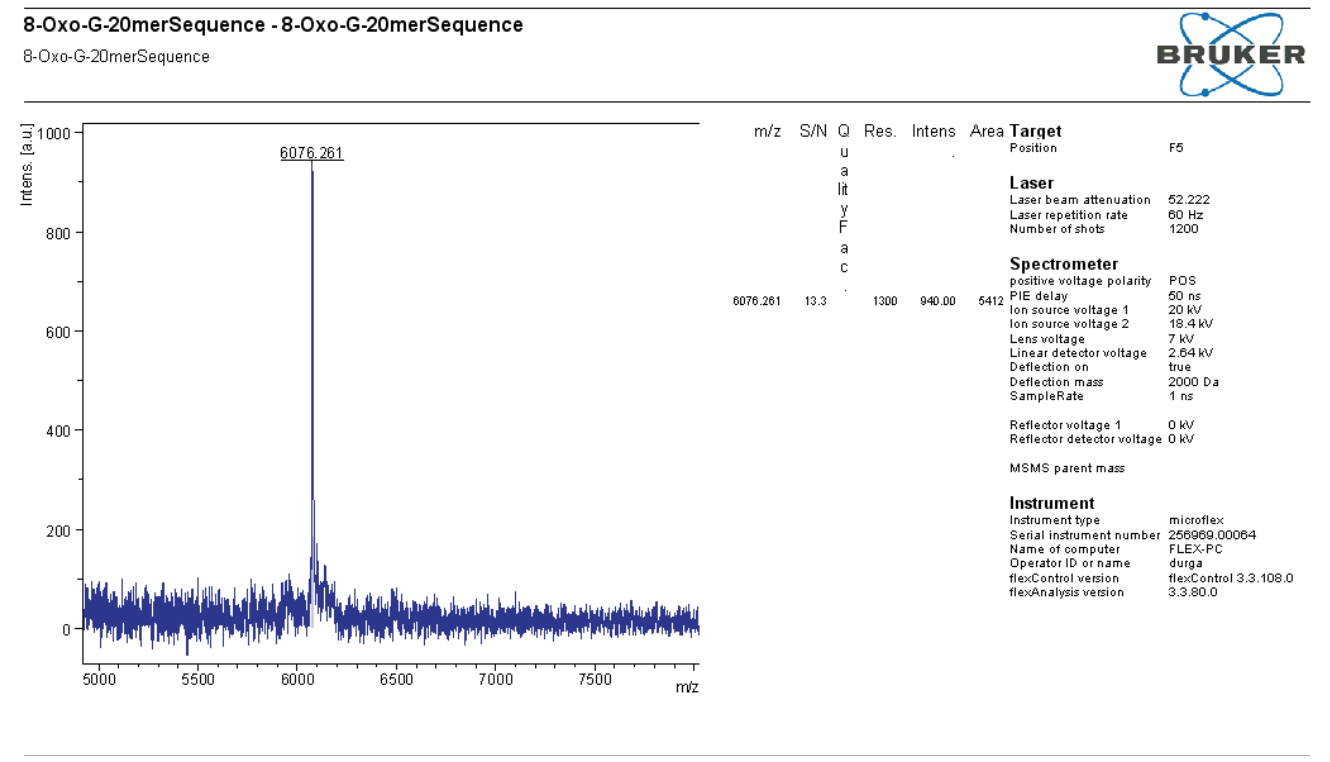

Figure A.18. MALDI-TOF MS of ODN 2.24 


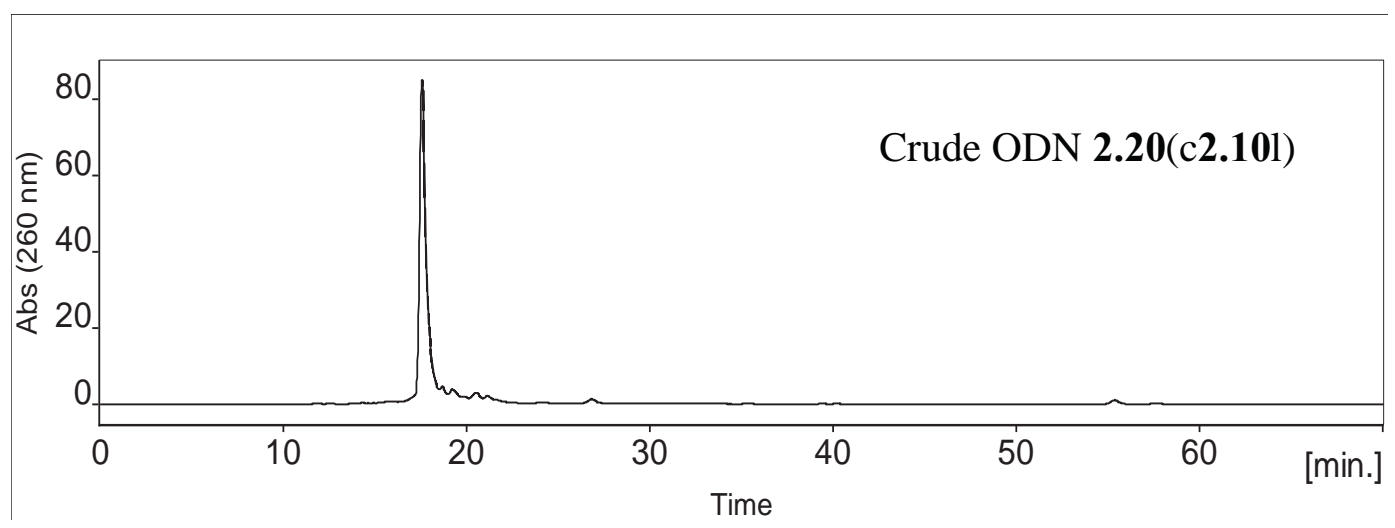

Figure A.19. RP HPLC profile of crude ODN 2.20(c2.101)

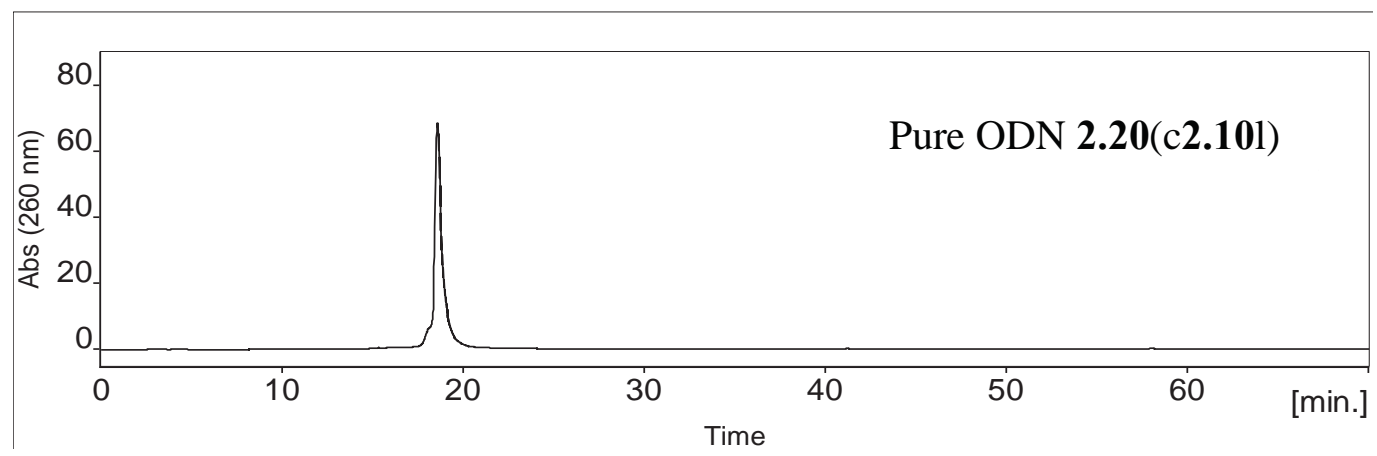

Figure A.20. RP HPLC profile of ODN 2.20(c2.101) purified by catching by polymerization 


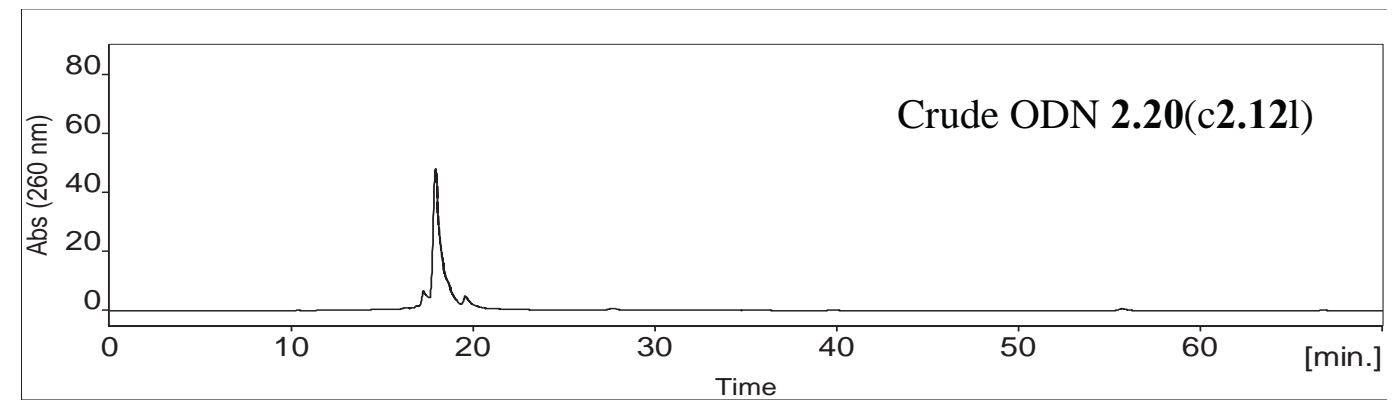

Figure A.21. RP HPLC profile of crude ODN 2.20(c2.121)

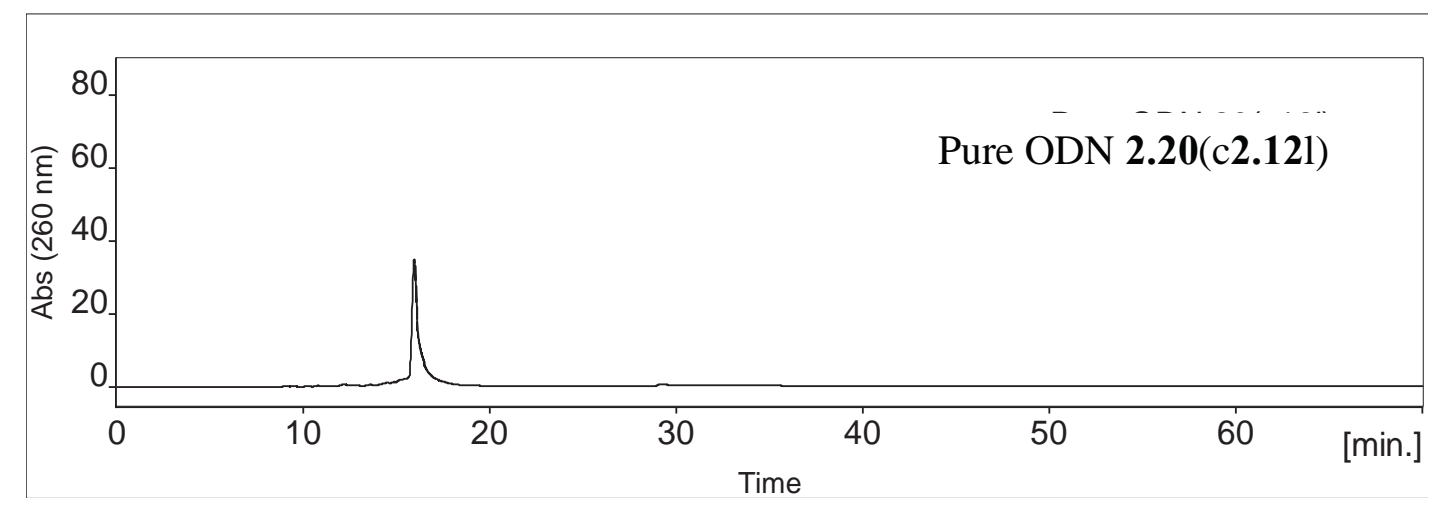

Figure A.22. HPLC profile of ODN 2.20(c2.121) purified by catching by polymerization 


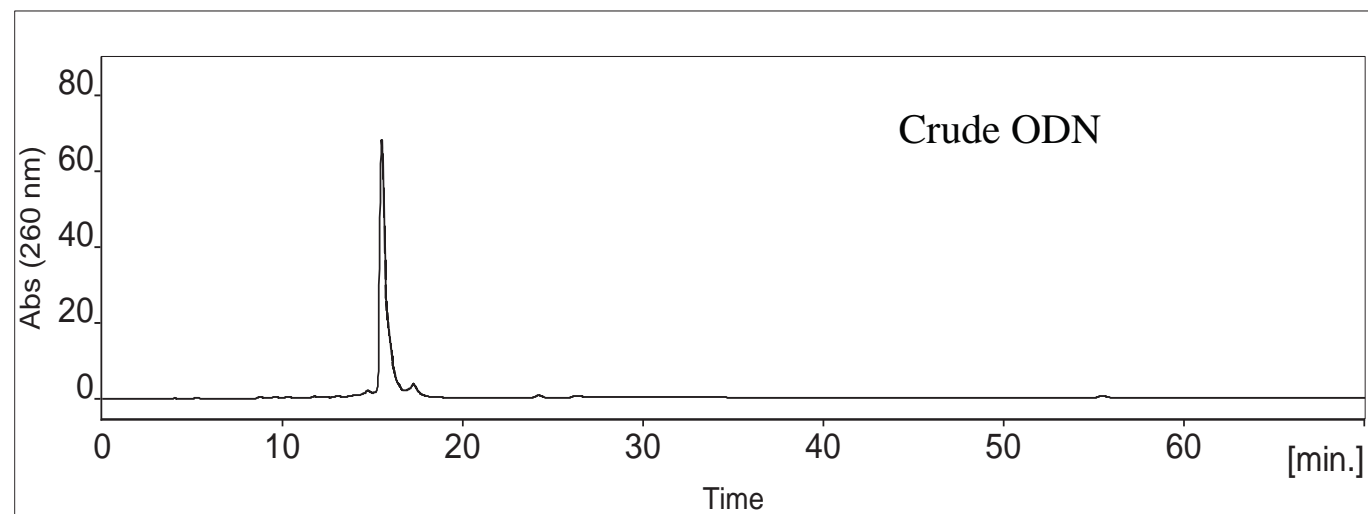

Figure A.23. RP HPLC profile of crude ODN 2.20(c2.131)

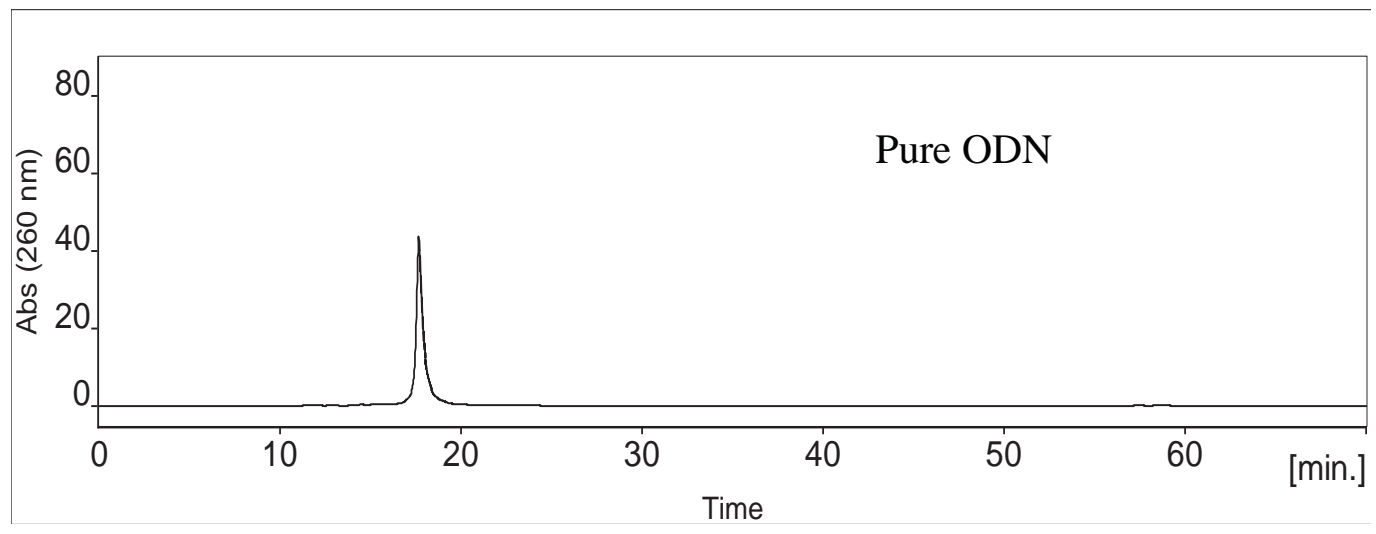

Figure A.24. RP HPLC profile of ODN 2.20(c2.131) purified by catching by polymerization 


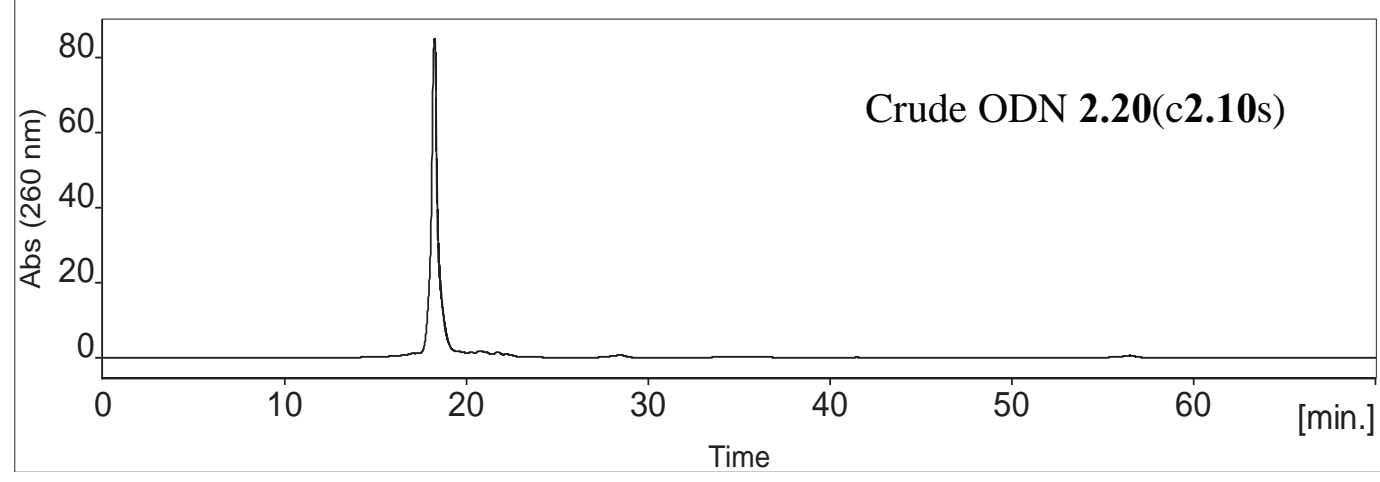

Figure A.25. RP HPLC profile of crude ODN 2.20(c2.10s)

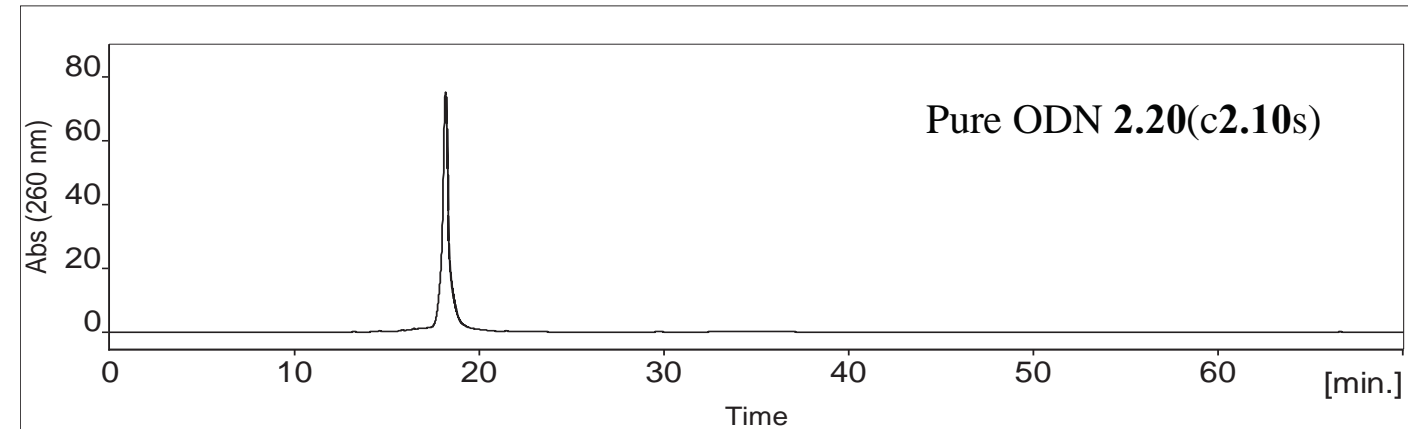

Figure A.26. RP HPLC profile of ODN 2.20(c2.10s) purified by catching by polymerization 


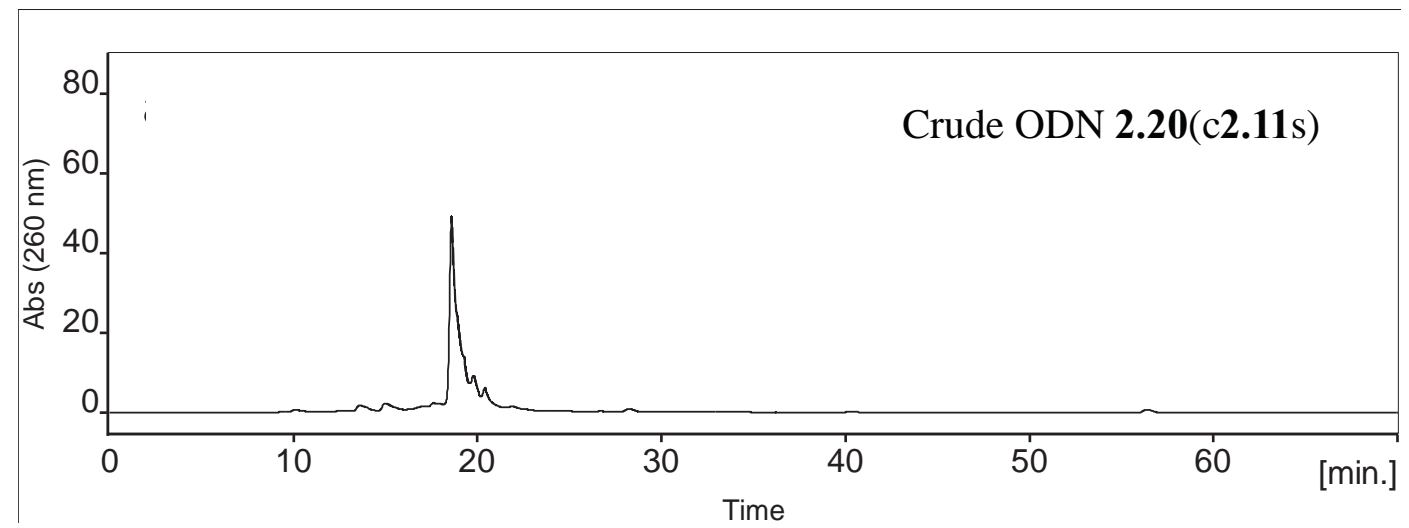

Figure A.27. RP HPLC profile of crude ODN 2.20(c2.11s)

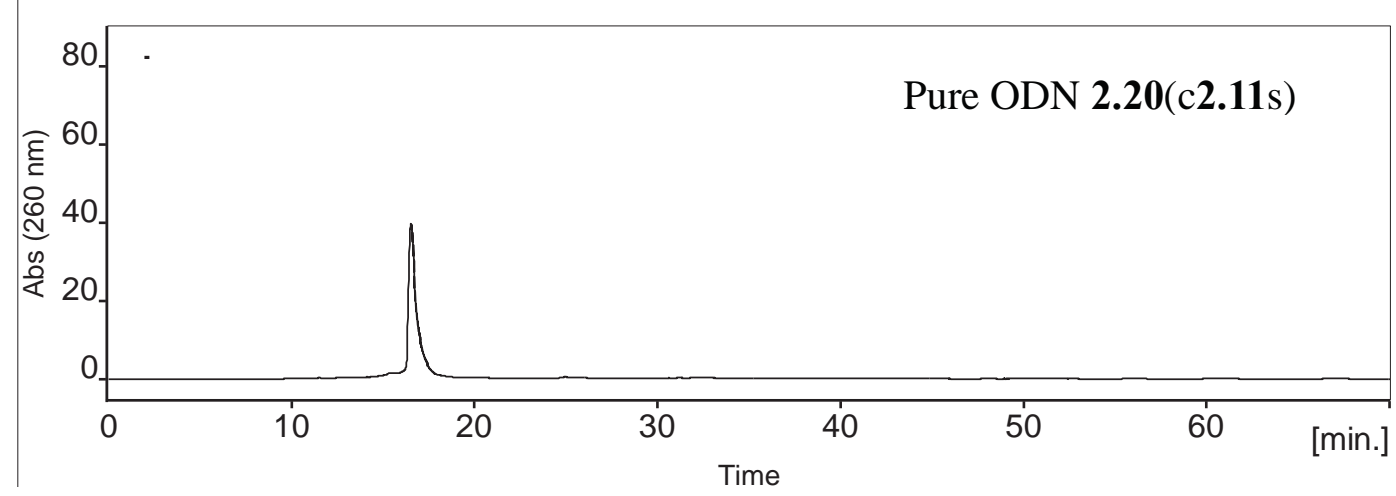

Figure A.28. RP HPLC profile of ODN 2.20(c2.11s) purified by catching by polymerization 


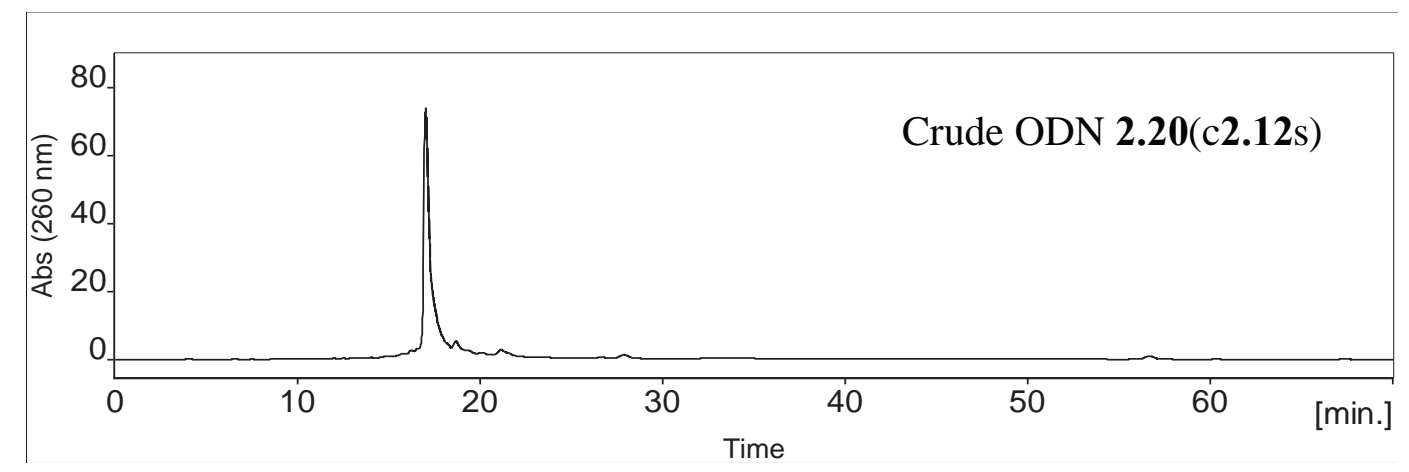

Figure A.29. RP HPLC profile of crude ODN 2.20(c2.12s)

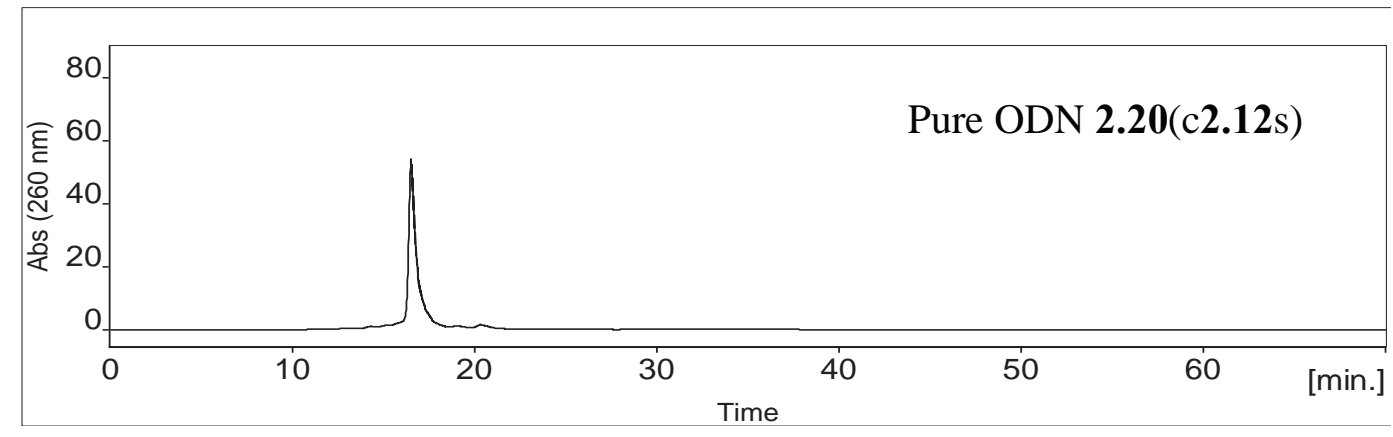

Figure A.30. RP HPLC profile of ODN 2.20(c2.12s) purified by catching by polymerization 


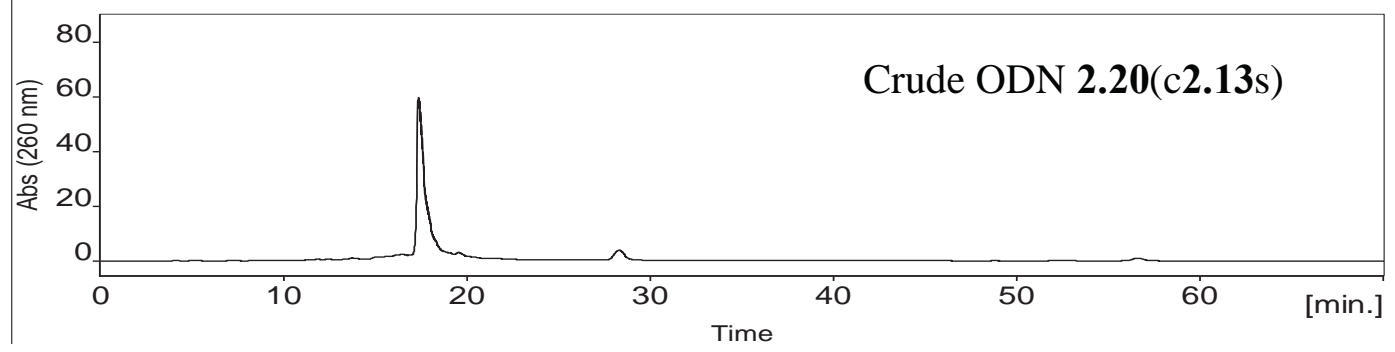

Figure A.31. RP HPLC profile of crude ODN 2.20(c2.13s)

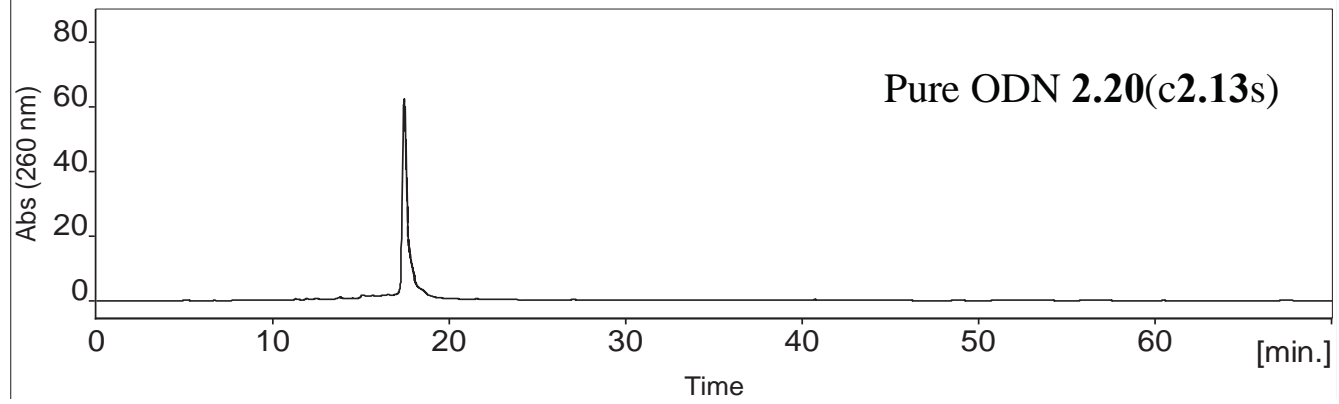

Figure A.32. RP HPLC profile of ODN 2.20(c13s) purified by catching by polymerization 


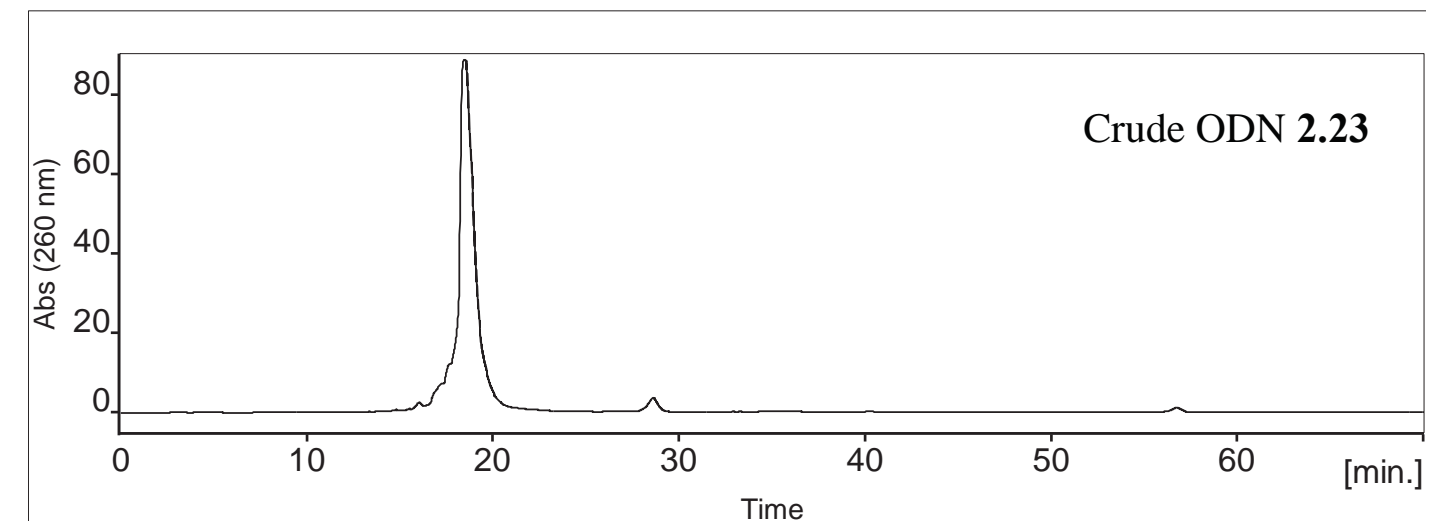

Figure A.33. RP HPLC profile of crude ODN $\mathbf{2 . 2 3}$

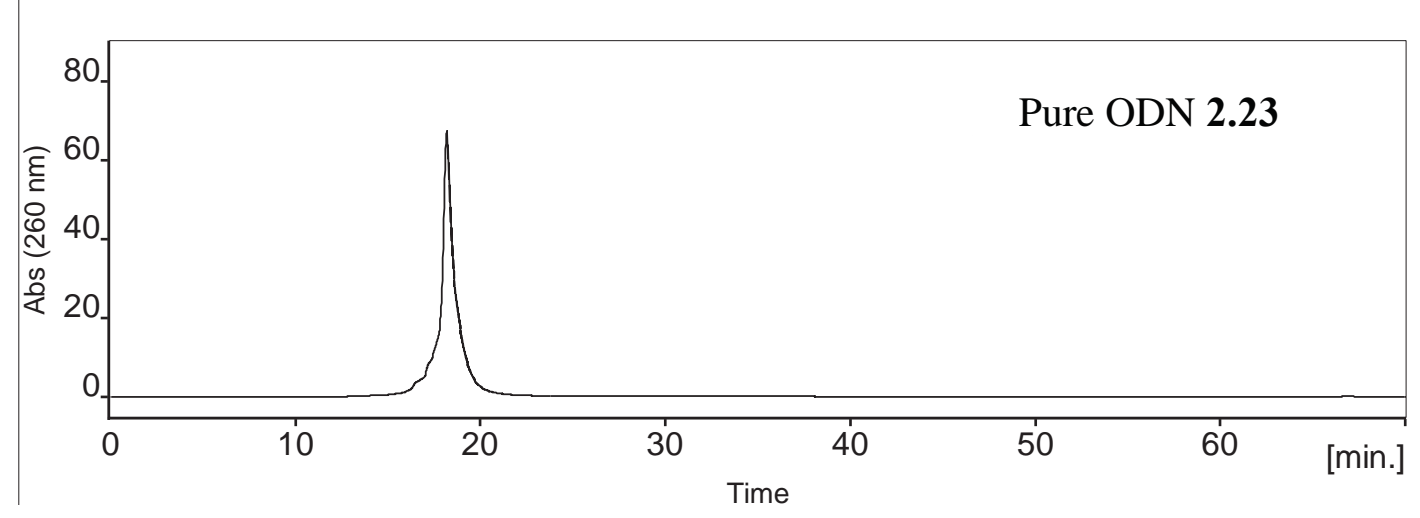

Figure A.34. RP HPLC profile of ODN 2.23 purified by catching by polymerization 


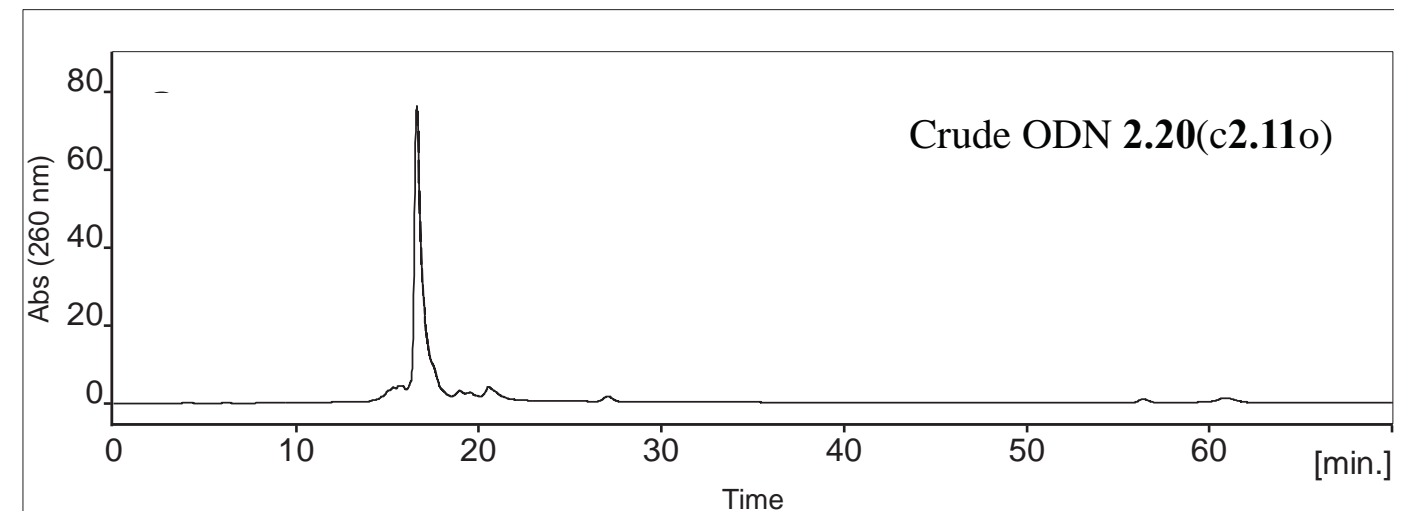

Figure A.35. RP HPLC profile of crude ODN 2.20(c2.11o)

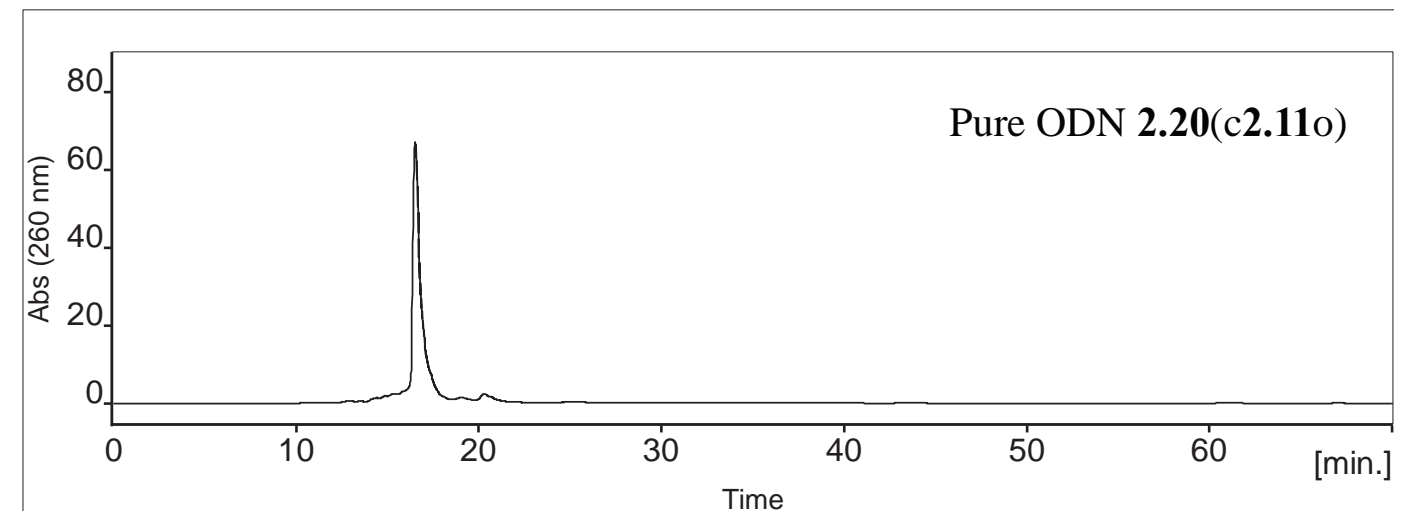

Figure A.36. RP HPLC profile of ODN 2.20(c2.11o) purified by catching by polymerization 


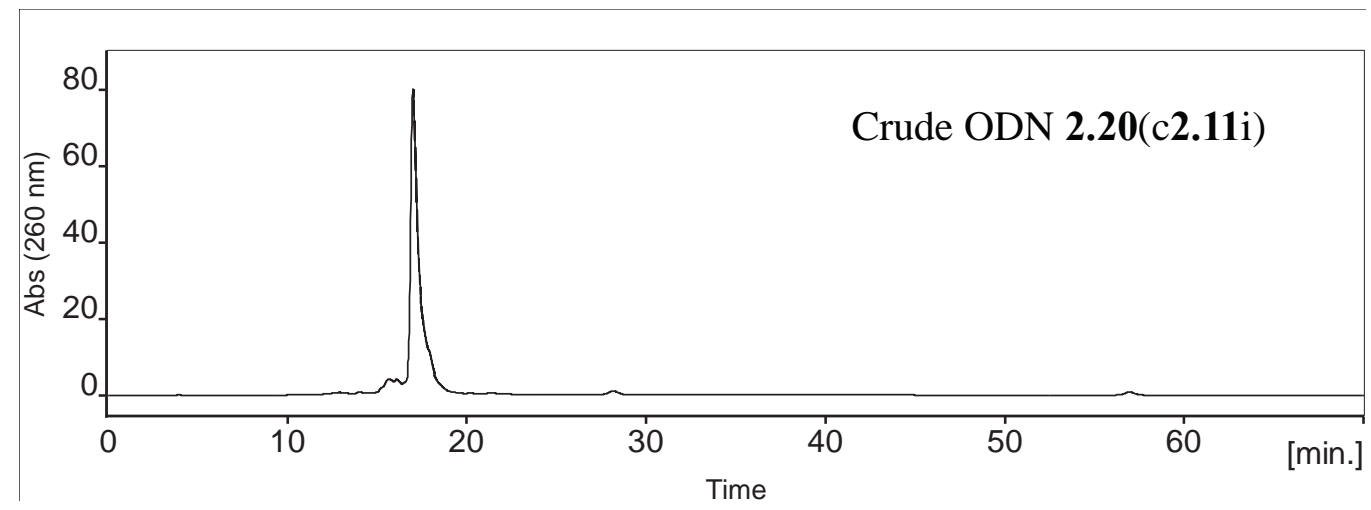

Figure A.37. RP HPLC profile of crude ODN 2.20(c2.21i)

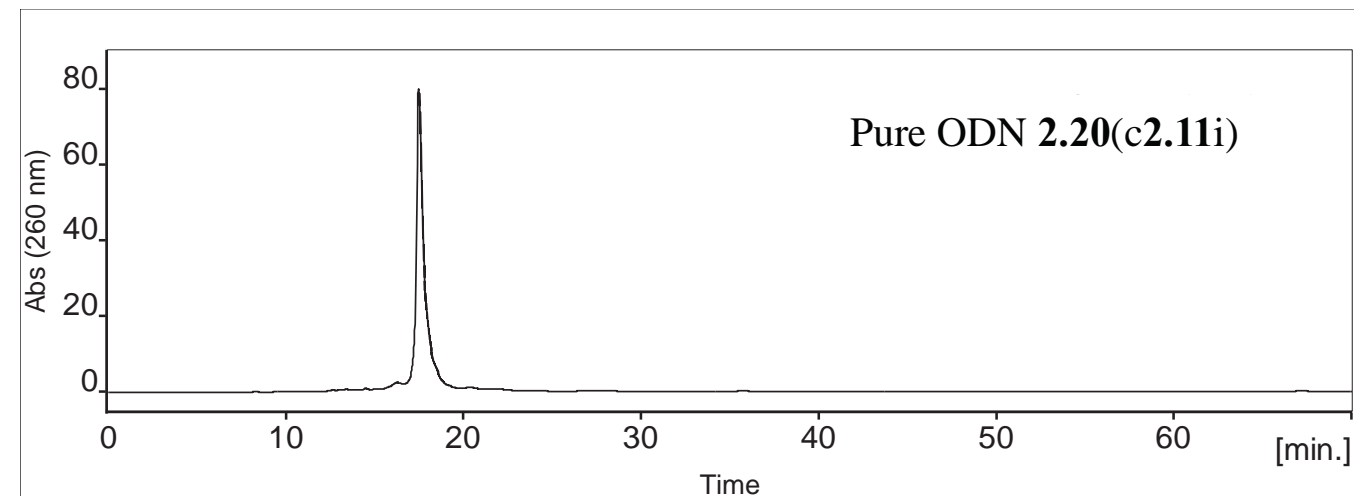

Figure A.38. RP HPLC profile of ODN 2.20(c2.11i) purified by catching by polymerization 


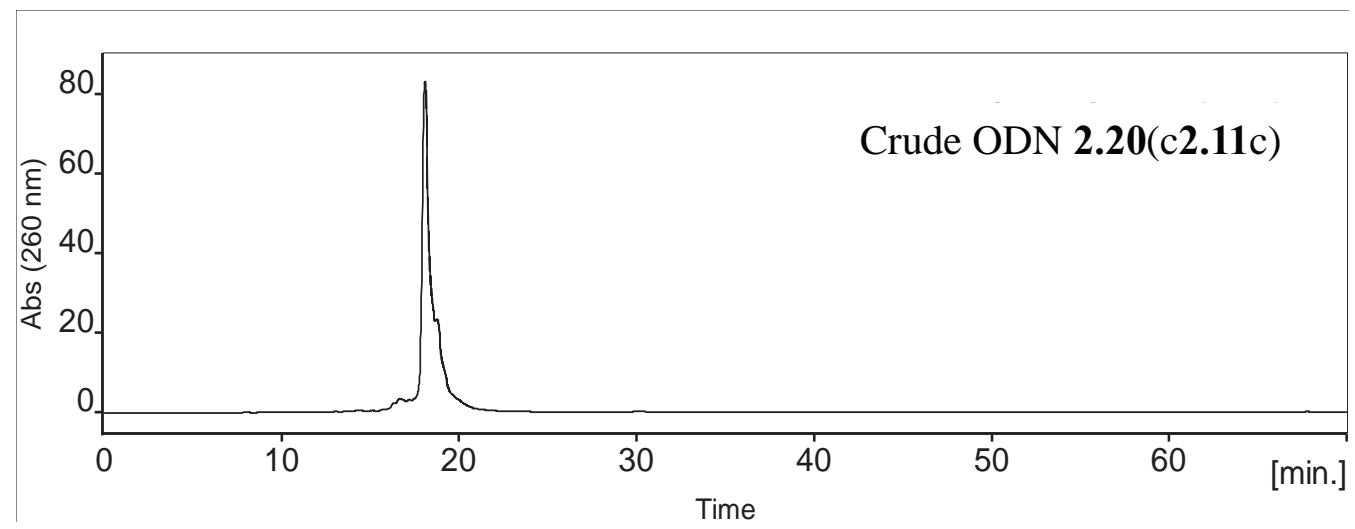

Figure A.39. RP HPLC profile of crude ODN 2.20(c2.11c)

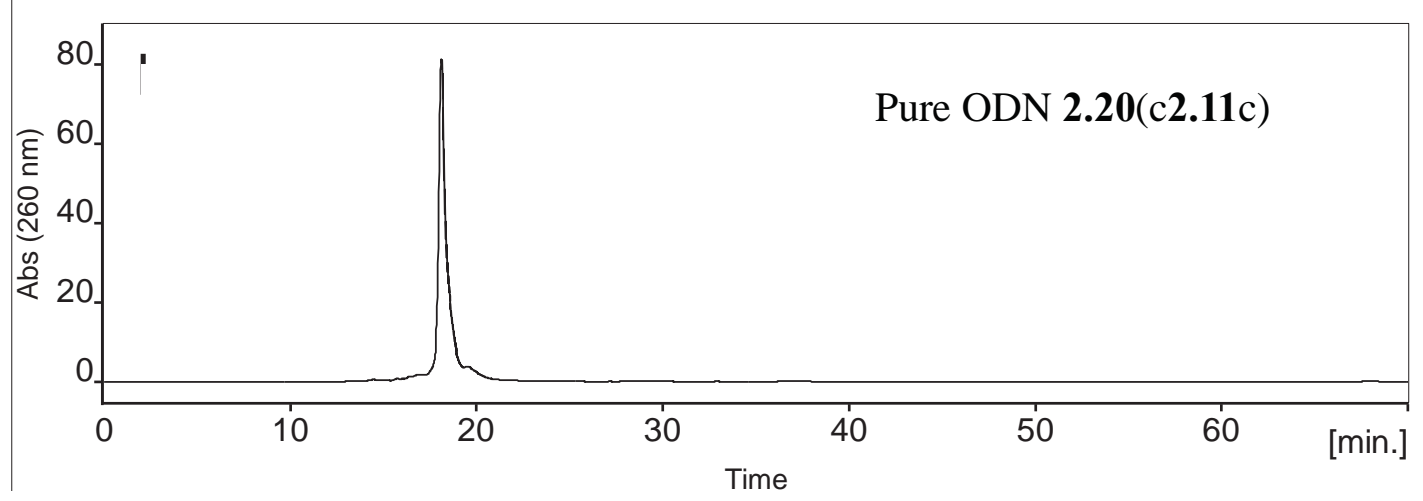

Figure A.40. RP HPLC profile of ODN 2.20(c2.11c) purified by catching by polymerization 


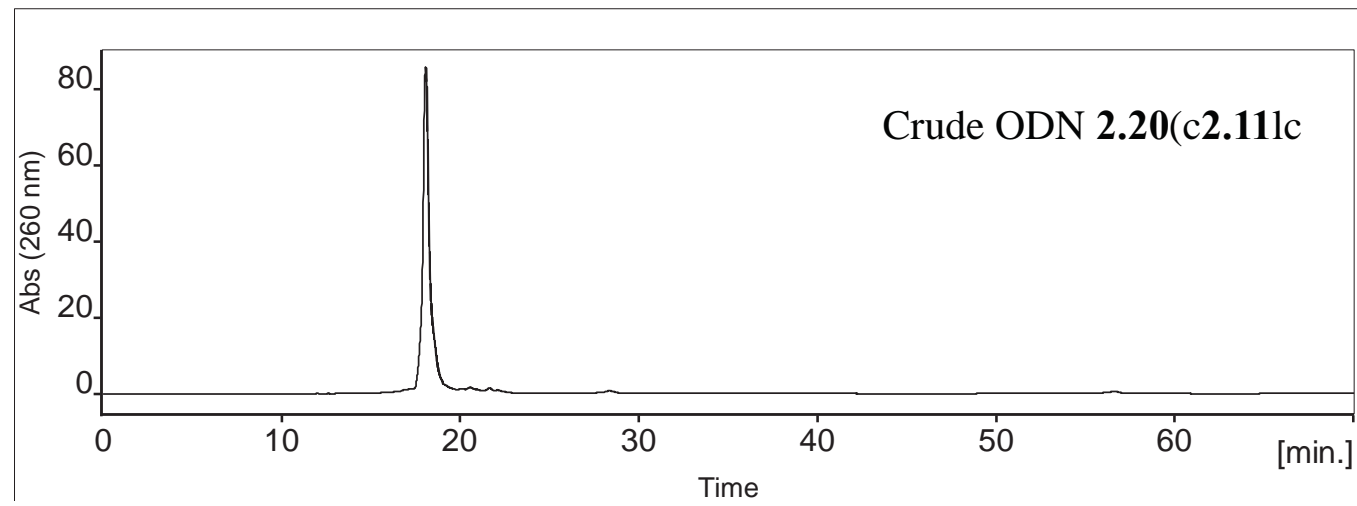

Figure A.41. RP HPLC profile of crude ODN 2.20(c2.11lc)

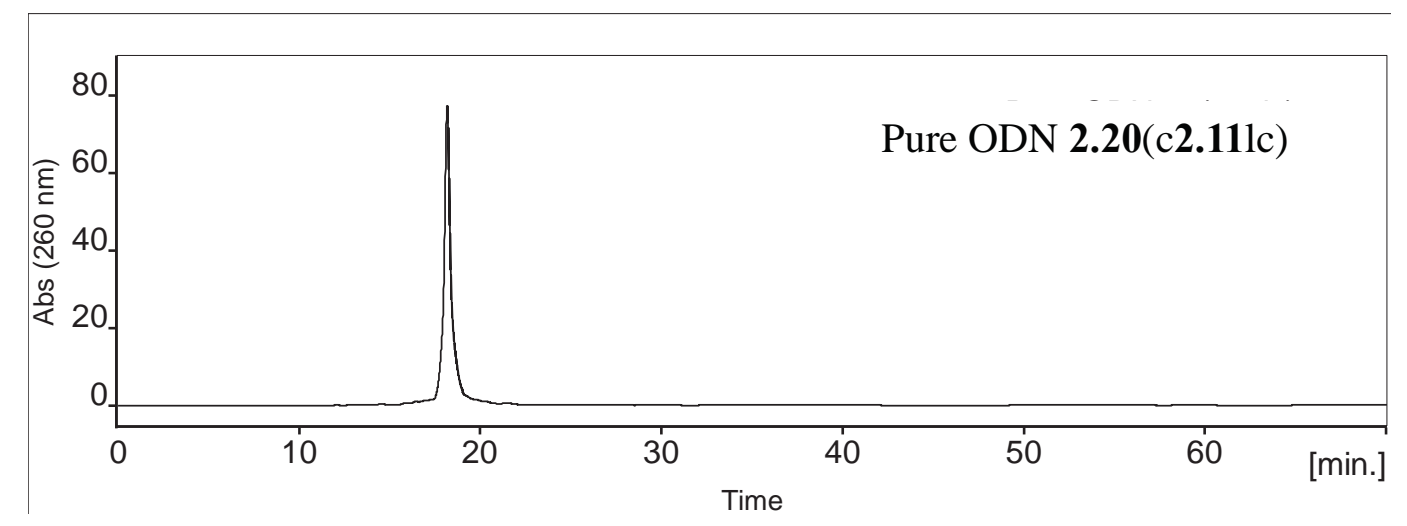

Figure A.42. RP HPLC profile of ODN 2.20(c2.11lc) purified by catching by polymerization 


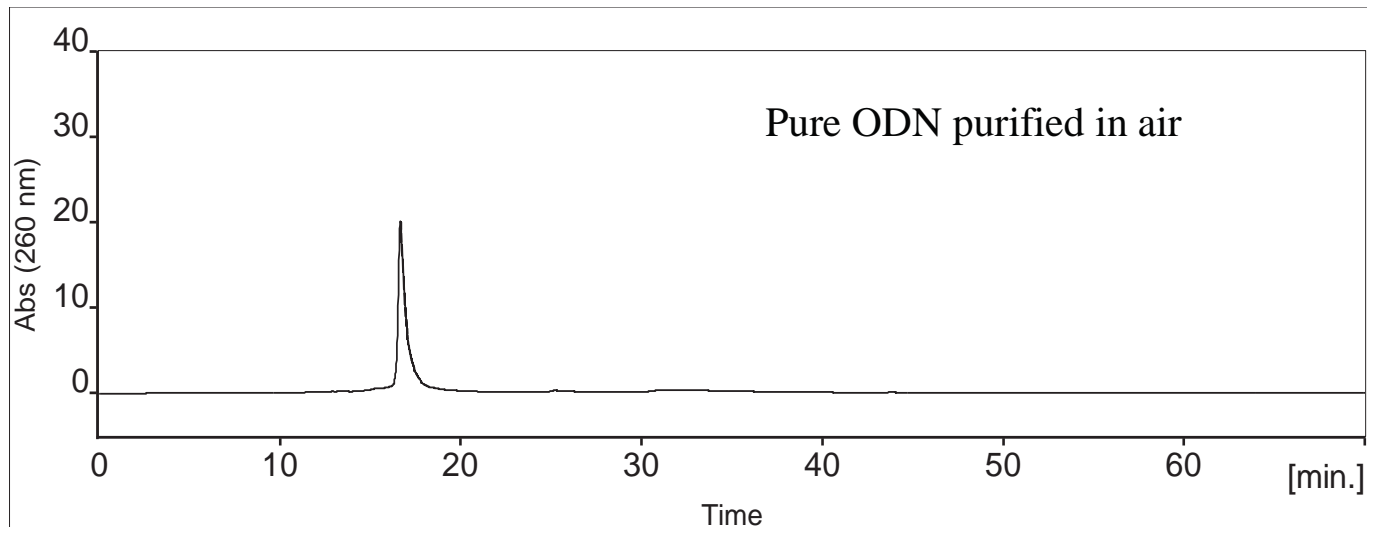

Figure A.43. RP HPLC profile of ODN 2.20 purified by catching by polymerization; the polymerization step was carried in air in a centrifuge tube

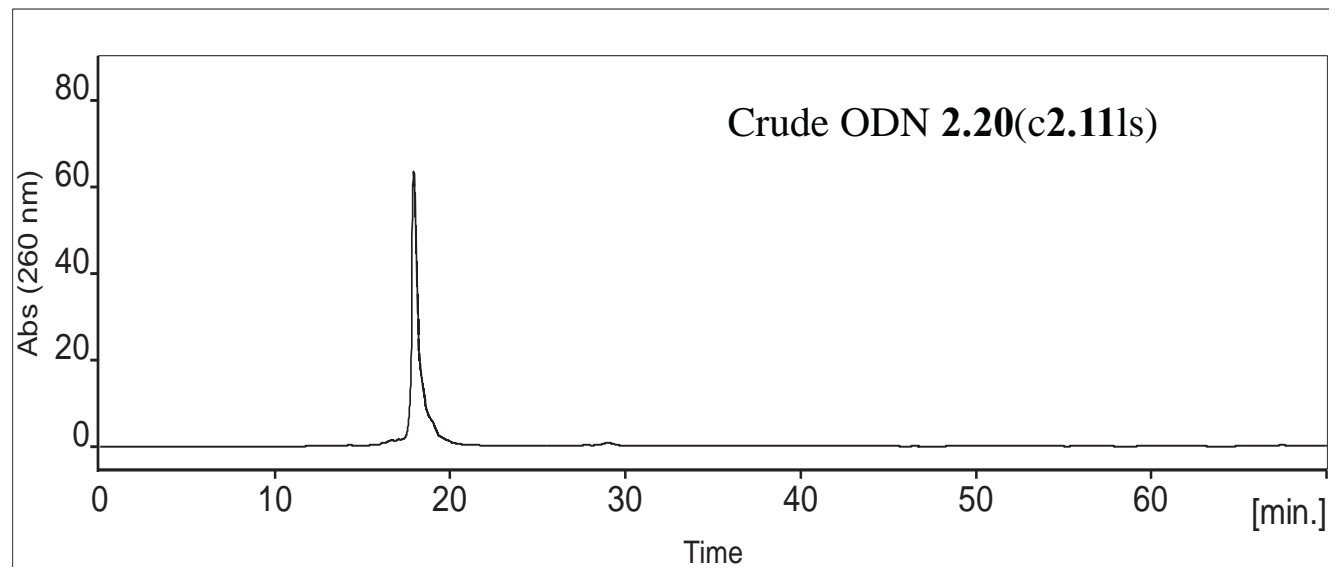

Figure A.44. RP HPLC profile of crude ODN 2.20(c2.11ls) 


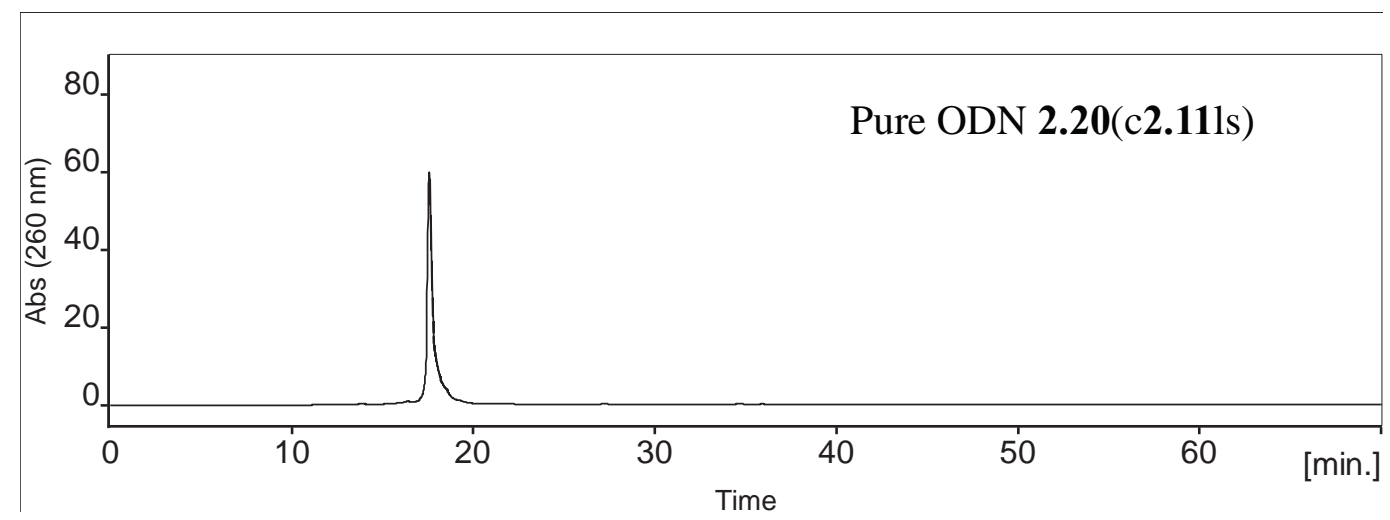

Figure A.45. RP HPLC profile of ODN 2.20(c2.11ls) purified by catching by polymerization

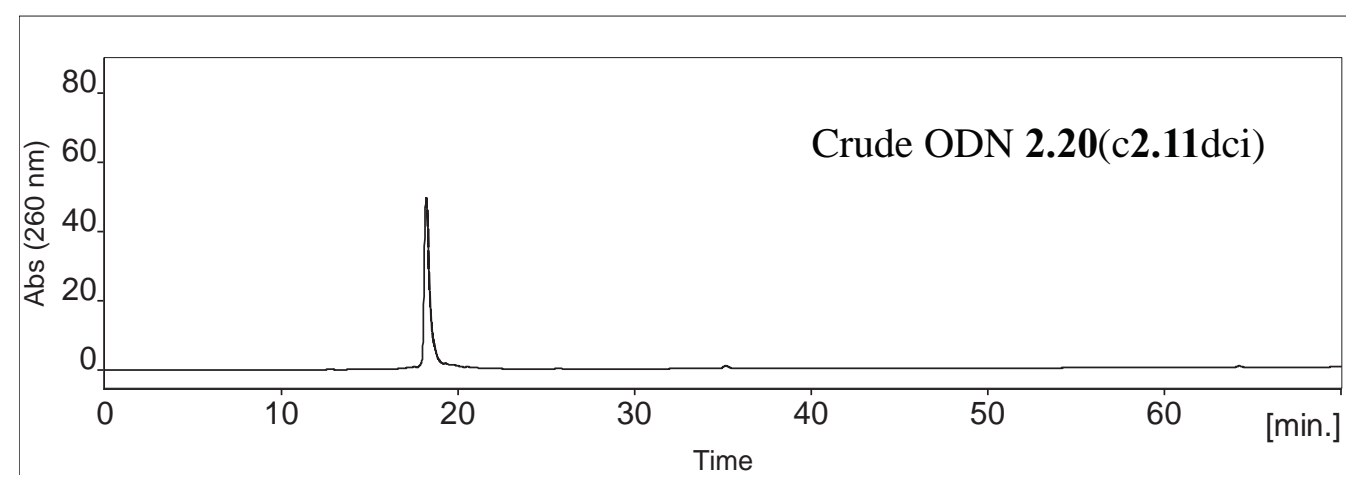

Figure A.46. RP HPLC profile of crude ODN 2.20(c2.11dci) purified at $3 \mu \mathrm{mol}$ scale using the catching failure sequences by polymerization method 


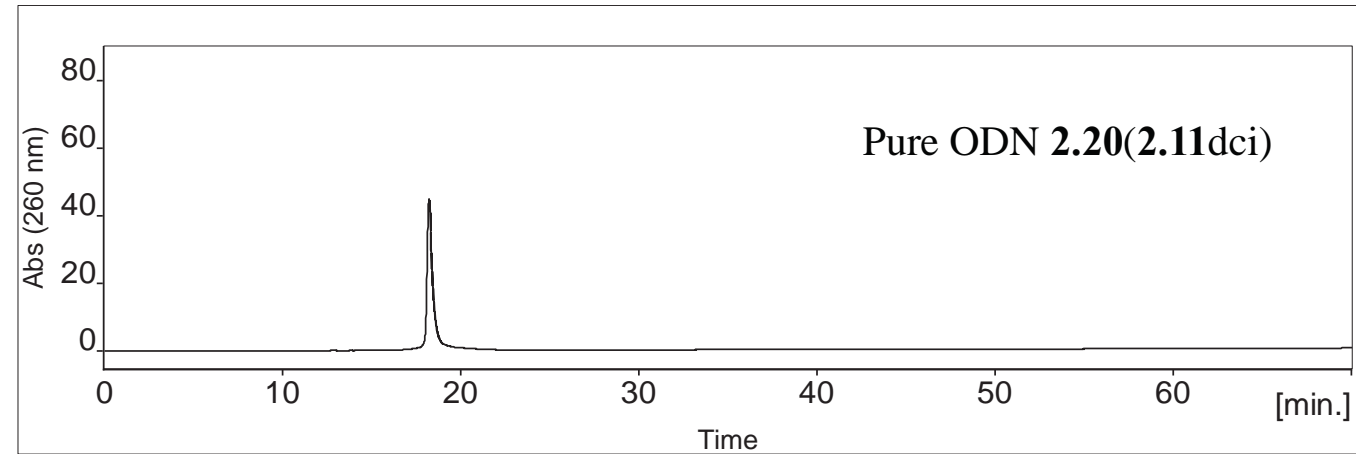

Figure A.47. RP HPLC profile of ODN 2.20 (c2.11dci) purified at $3 \mu \mathrm{mol}$ scale using the catching failure sequences by polymerization method

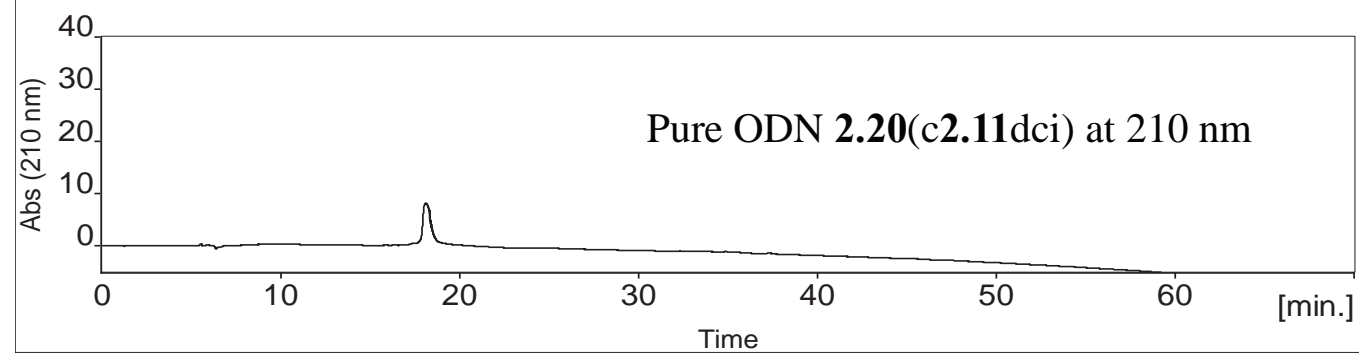

Figure A.48. RP HPLC profile of ODN 2.20(c2.11dci) purified at $3 \mu \mathrm{mol}$ scale using the catching failure sequences by polymerization method; the profile was generated by UV detection at $210 \mathrm{~nm}$ 


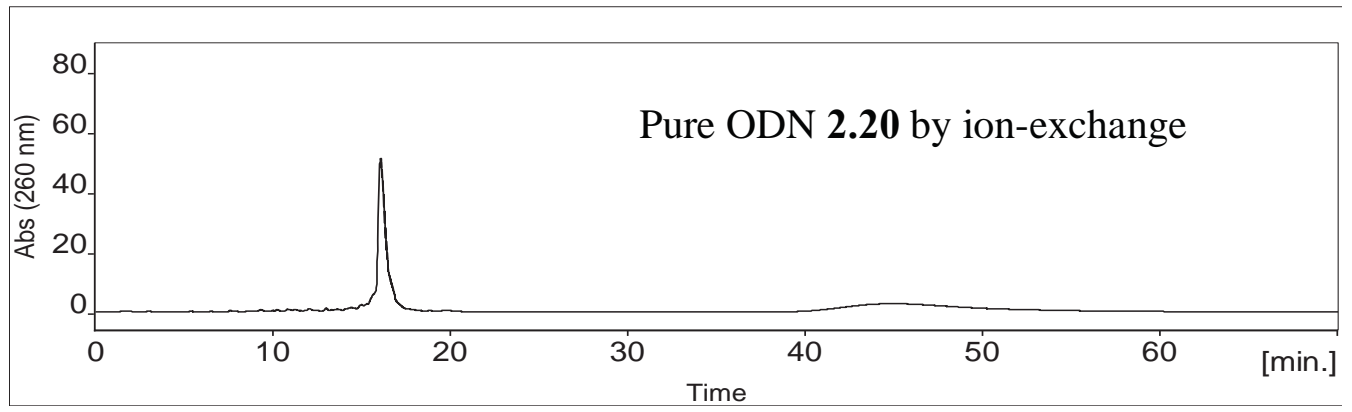

Figure A.49. Ion-exchange HPLC analysis of ODN 2.20. The conditions described in the general methods section were used. For analysis, $20 \mu \mathrm{L}$ solution from the remaining ODN $2.20(\mathrm{c} 2.12 \mathrm{~s})$ solution in the purification by catching failure sequences by polymerization experiment following the general ODN purification procedure was injected into HPLC.

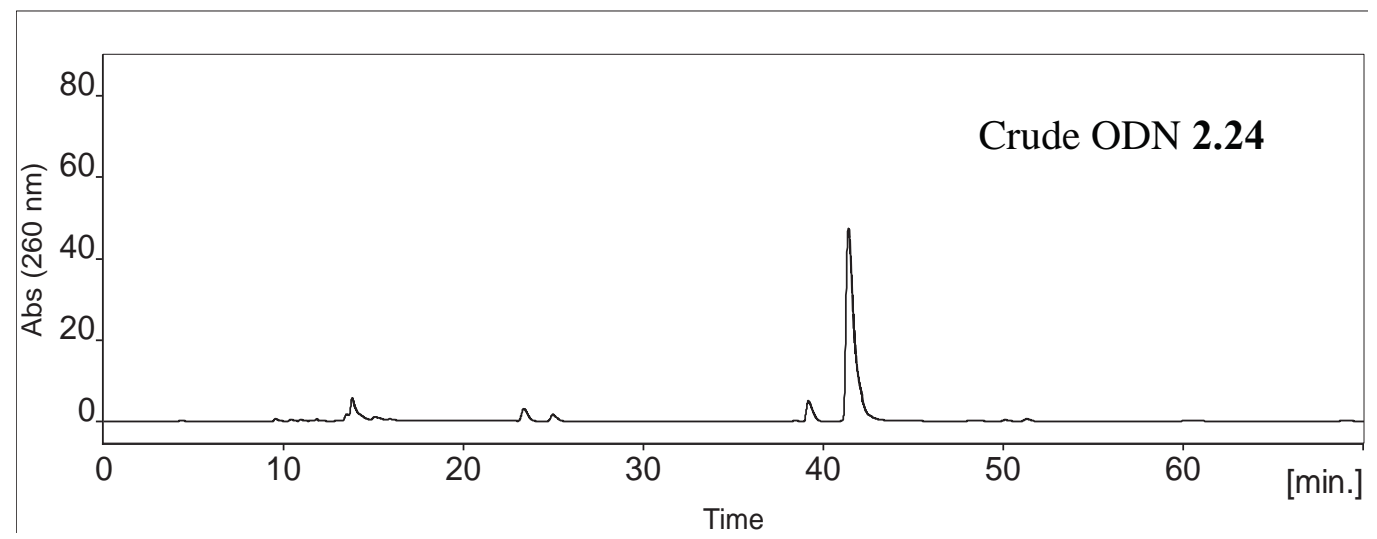

Figure A.50. RP HPLC profile of crude ODN 2.24 


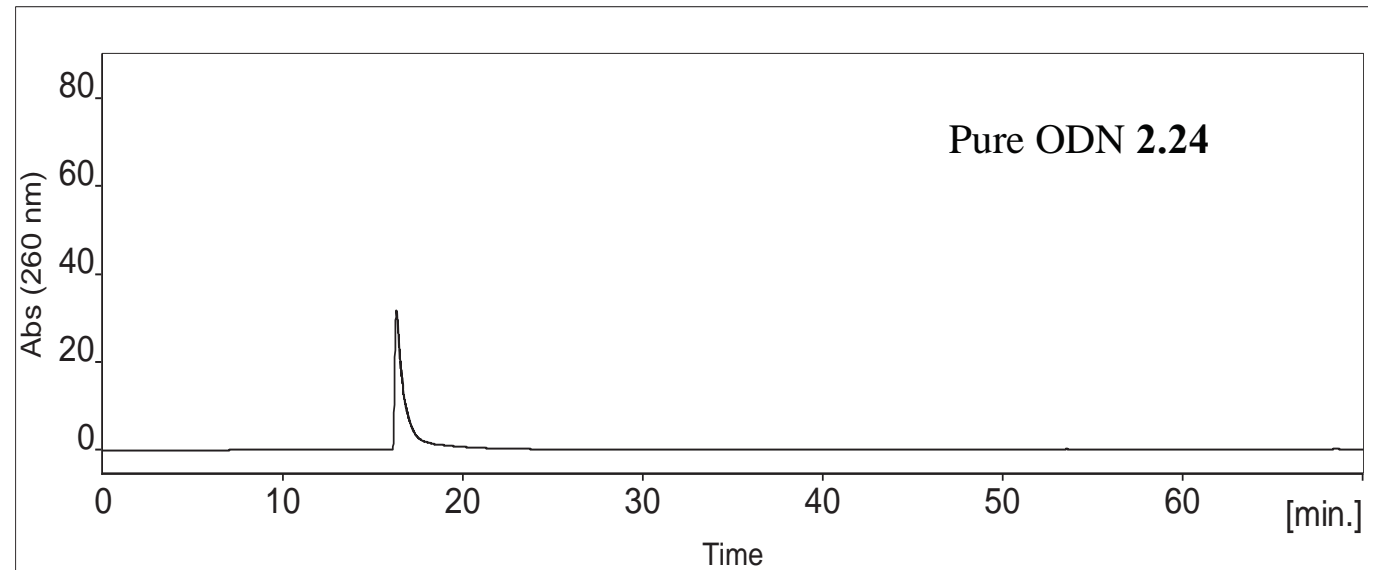

Figure A.51. RP HPLC profile of pure ODN 2.24

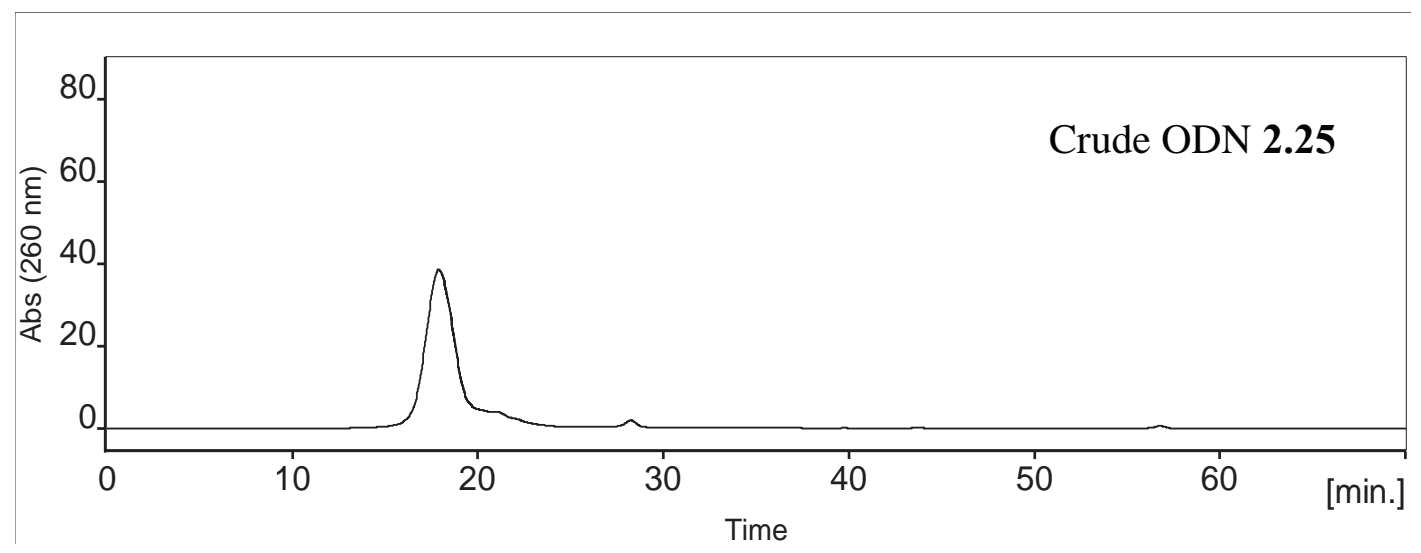

Figure A.52. RP HPLC profile of crude ODN mixture $\mathbf{2 . 2 5}$ 


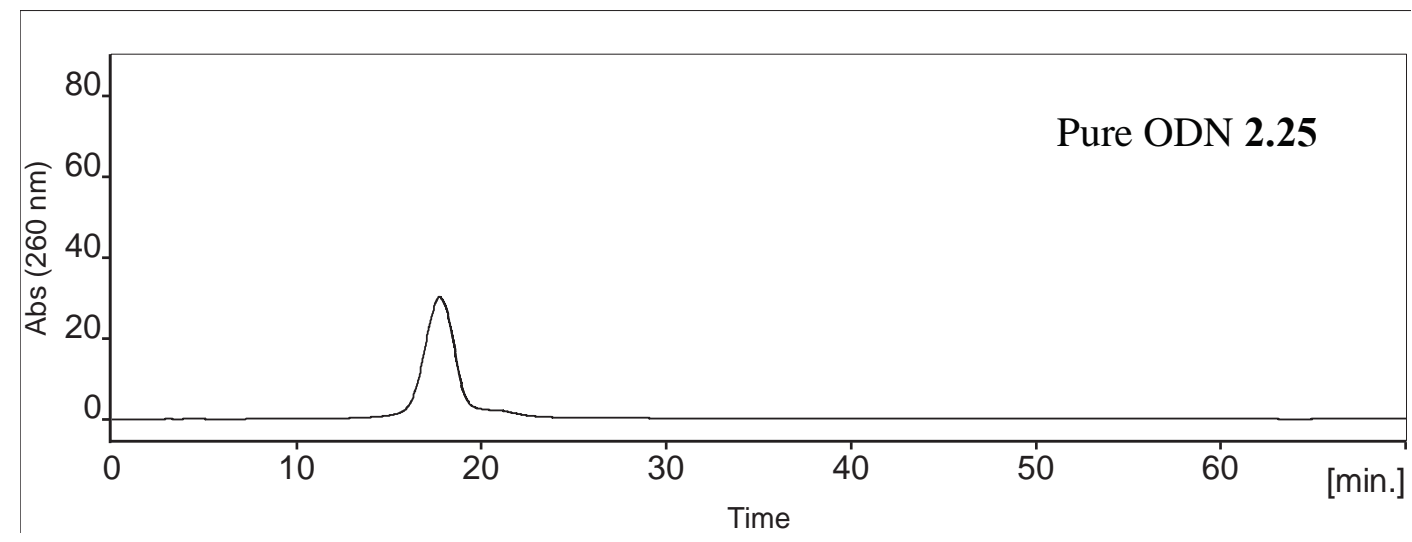

Figure A.53. RP HPLC profile of ODN 2.25 that went through catching by polymerization

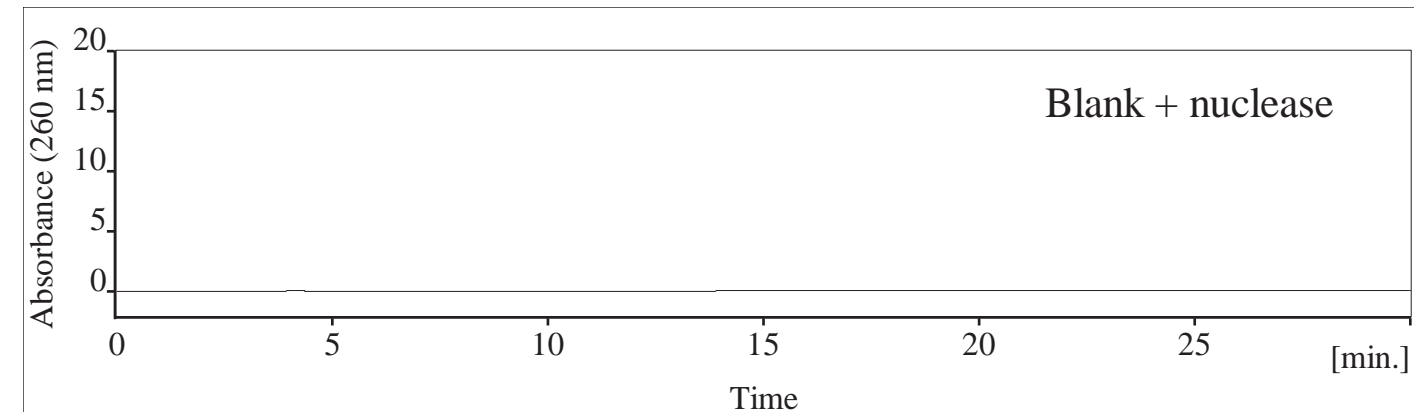

Figure A.54. RP HPLC profile of nucleosides from ODN enzymatic digestion essay for ODNs that do not contain 8-oxo-dG-the blank control 


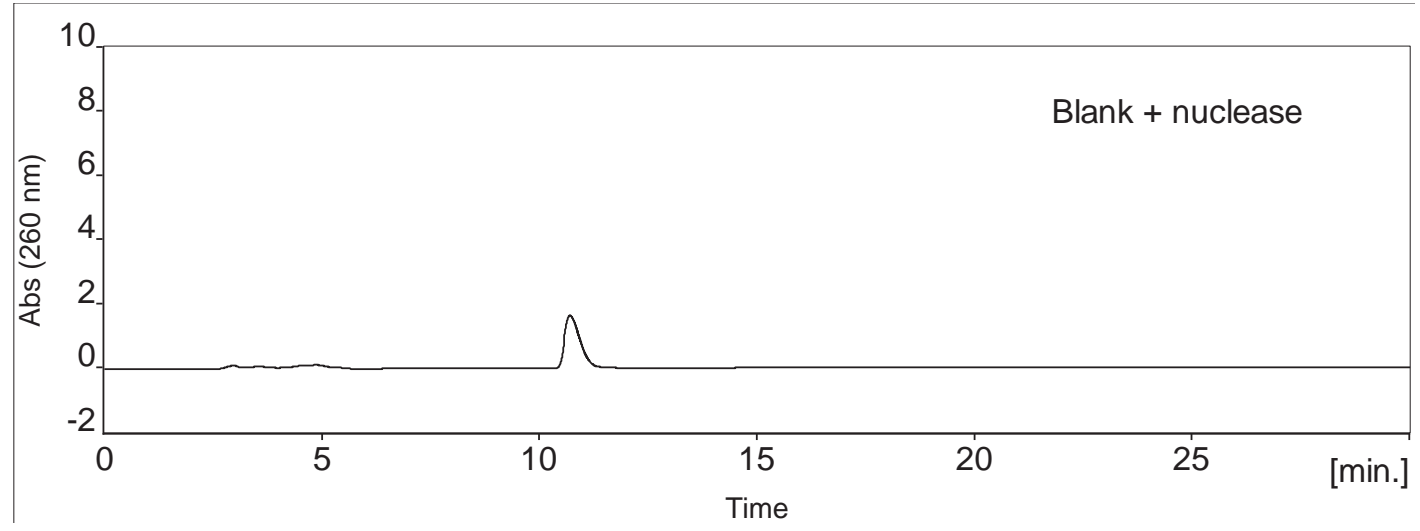

Figure A.55. RP HPLC profile of nucleosides from ODN enzymatic digestion essay for ODN that contain 8-oxo-dG-the blank control; the peak at 11 minutes is resulted from 2-mercaptoethanol added to prevent oxidation of 8-oxo-dG 
Table A.1. Nucleoside peak areas in HPLC profiles of SVP and BAP digested ODNs

\begin{tabular}{l|cccc|cccc|cccccc}
\hline \hline ODN & \multicolumn{4}{|c|}{$\mathbf{2 0}$} & \multicolumn{5}{c|}{ Authentic 20 } & \multicolumn{7}{c}{$\mathbf{2 4}$} \\
\hline Base & $\mathrm{dC}$ & $\mathrm{dG}$ & $\mathrm{dT}$ & $\mathrm{dA}$ & $\mathrm{dC}$ & $\mathrm{dG}$ & $\mathrm{dT}$ & $\mathrm{dA}$ & $\mathrm{dC}$ & $\mathrm{dG}$ & $\mathrm{dT}$ & $\mathrm{dA}$ & $\begin{array}{c}8- \\
\text { Oxo- } \\
\mathrm{dG}\end{array}$ \\
\hline $\begin{array}{l}\text { Number } \\
\text { of base } \\
\begin{array}{l}\text { Calcd } \\
\text { relative } \\
\text { area* }\end{array}\end{array}$ & 6 & 4 & 7 & 3 & 6 & 4 & 7 & 3 & 6 & 3 & 7 & 3 & 1 \\
$\begin{array}{l}\text { Meaured } \\
\text { relative } \\
\text { area }\end{array}$ & 6.0 & 6.4 & 8.5 & 6.3 & 6.0 & 6.4 & 8.5 & 6.3 & 6.0 & 4.8 & 8.5 & 6.3 & - \\
\hline \hline
\end{tabular}

* Calculated based on the relative nucleoside extinction coefficients of $\mathrm{dC} / \mathrm{dG} / \mathrm{dT} / \mathrm{dA}$

1.0:1.6:1.2:2.1 from Current Protocols in Nucleic Acid Chemistry, Unit 10.6, DOI: 10.1002/0471142700.nc1006s01.

Table A.2. Recipes for the preparation of polymerization solutions

\begin{tabular}{c|cc|cc|c}
\hline \multirow{2}{*}{$\begin{array}{c}\text { Cross-linking } \\
\text { ratio }\end{array}$} & \multicolumn{2}{|c|}{$N, N^{\prime}$-Methylenebis(acrylamide) } & \multicolumn{2}{|c|}{$N, N$-Dimethylacrylamide } & \multirow{2}{*}{ Water $(\mathrm{mL})$} \\
\cline { 2 - 5 } & Mass (mmol) & Weight $(\mathrm{mg})$ & Mass $(\mathrm{mmol})$ & Volume $(\mu \mathrm{L})$ & \\
\hline $1: 50$ & 0.169 & 26.05 & 8.44 & 870 & 5 \\
$1: 25$ & 0.337 & 52.1 & 8.44 & 870 & 5 \\
$1: 15$ & 0.563 & 86.8 & 8.44 & 870 & 5 \\
$1: 7$ & 1.207 & 186.1 & 8.44 & 870 & 5 \\
$1: 2$ & 4.225 & 651.3 & 8.44 & 870 & $14^{*}$ \\
\hline
\end{tabular}

* More water had to be added to dissolve the materials. 
Table A.3. ODN synthetic cycles using polymerizable phosphoramidite as capping agent

\section{Cycle $1-0.2 \mu \mathrm{mol}$ synthesis, the general ODN synthesis procedure}

ODN synthetic cycle using polymerizable phosphoramidite as the capping agent

Synthesizer: standard ABI 394 solid phase synthesizer; 4-column 8-base instrument Polymerizable capping agent: $0.15 \mathrm{M}$ solution of capping phosphoramidite in acetonitrile, placed at the bottle 5 position

The bottles for normal $\mathrm{Ac}_{2} \mathrm{O}$ capping agents are empty

Activator for the capping phosphoramidite: from the same bottle for the coupling steps

Synthesis scale: $0.2 \mu \mathrm{mol}$

Column used: column 2; functions for other columns are not shown

\begin{tabular}{llll}
\hline Step number & Function number & Function name & Step time \\
\hline 1. & 106 & Begin & \\
2. & 64 & 18 To waste & 3.0 \\
3. & 42 & 18 To column & 10.0 \\
4. & 2 & Reverse flush & 8.0 \\
5. & 1 & Block flush & 4.0 \\
6. & 101 & Phos Prep & 3.0 \\
7. & 142 & Column 2 on & \\
8. & 111 & Block vent & 2.0 \\
9. & Tet to waste & 1.7 \\
10. & 38 & B+Tet to column & 2.0 \\
11. & 34 & Tet to column & 1.0 \\
12. & 33 & B+Tet to column & 1.5 \\
13. & 43 & Push to column & \\
14. & 143 & Column 2 off & \\
15. & 103 & wait & 25.0 \\
16. & 64 & 18 To waste & 3.0 \\
17. & 42 & 18 To column & 10.0 \\
18. & 2 & Reverse flush & 8.0 \\
& & 214 &
\end{tabular}




\begin{tabular}{|c|c|c|c|}
\hline 19. & 1 & Block flush & 4.0 \\
\hline 20. & 101 & Phos Prep & 3.0 \\
\hline 21. & 142 & Column 2 on & \\
\hline 22. & 111 & Block vent & 2.0 \\
\hline 23. & 58 & Tet to waste & 1.7 \\
\hline 24. & 35 & $5+$ Tet to column & 2.0 \\
\hline 25. & 34 & Tet to column & 1.0 \\
\hline 26. & 103 & Wait & 90.0 \\
\hline 27. & 35 & $5+$ Tet to column & 1.5 \\
\hline 28. & 103 & Wait & 90.0 \\
\hline 29. & 35 & $5+$ Tet to column & 1.5 \\
\hline 30. & 103 & Wait & 90.0 \\
\hline 31. & 35 & $5+$ Tet to column & 1.5 \\
\hline 32. & 103 & Wait & 90.0 \\
\hline 33. & 43 & Push to column & \\
\hline 34. & 103 & Wait & 30.0 \\
\hline 35. & 64 & 18 To waste & 4.0 \\
\hline 36. & 42 & 18 To column & 8.0 \\
\hline 37. & 4 & Flush to waste & 4.0 \\
\hline 38. & 42 & 18 To column & 8.0 \\
\hline 39. & 2 & Reverse flush & 5.0 \\
\hline 40. & 42 & 18 To column & 8.0 \\
\hline 41. & 2 & Reverse flush & 5.0 \\
\hline 42. & 1 & Block flush & 3.0 \\
\hline 43. & 41 & 15 To column & 8.0 \\
\hline 44. & 64 & 18 To waste & 4.0 \\
\hline 45. & 1 & Block flush & 3.0 \\
\hline 46. & 103 & Wait & 10.0 \\
\hline 47. & 41 & 15 To column & 8.0 \\
\hline 48. & 64 & 18 To waste & 4.0 \\
\hline 49. & 1 & Block flush & 3.0 \\
\hline 50. & 103 & Wait & 10.0 \\
\hline 51. & 42 & 18 To column & 15.0 \\
\hline 52. & 4 & Flush to waste & 4.0 \\
\hline
\end{tabular}




\begin{tabular}{|c|c|c|c|}
\hline 53. & 42 & 18 To column & 10.0 \\
\hline 54. & 2 & Reverse flush & 5.0 \\
\hline 55. & 42 & 18 To column & 10.0 \\
\hline 56. & 2 & Reverse flush & 5.0 \\
\hline 57. & 1 & Block flush & 3.0 \\
\hline 58. & 105 & Start detrityl & \\
\hline 59. & 64 & 18 To waste & 4.0 \\
\hline 60. & 42 & 18 To column & 10.0 \\
\hline 61. & 2 & Reverse flush & 5.0 \\
\hline 62. & 1 & Block flush & 3.0 \\
\hline 63. & 167 & If monitoring & \\
\hline 64. & 44 & 19 To column & 25.0 \\
\hline 65. & 40 & 14 To column & 3.0 \\
\hline 66. & 135 & Monitor triyls & \\
\hline 67. & 40 & 14 To column & 25.0 \\
\hline 68. & 136 & Monitor noise & \\
\hline 69. & 40 & 14 To column & 10.0 \\
\hline 70. & 137 & Stop monitor & \\
\hline 71. & 42 & 18 To column & 10.0 \\
\hline 72. & 2 & Reverse flush & 8.0 \\
\hline 73. & 168 & If not monitoring & \\
\hline 74. & 40 & 14 To column & 6.0 \\
\hline 75. & 3 & Trityl flush & 5.0 \\
\hline 76. & 40 & 14 To column & 6.0 \\
\hline 77. & 103 & Wait & 5.0 \\
\hline 78. & 3 & Trityl flush & 5.0 \\
\hline 79. & 40 & 14 To column & 6.0 \\
\hline 80. & 103 & Wait & 5.0 \\
\hline 81. & 3 & Trityl flush & 5.0 \\
\hline 82. & 40 & 14 To column & 6.0 \\
\hline 83. & 103 & Wait & 5.0 \\
\hline 84. & 3 & Trityl flush & 5.0 \\
\hline 85. & 42 & 18 To column & 10.0 \\
\hline 86. & 3 & Trityl flush & 8.0 \\
\hline & & 216 & \\
\hline
\end{tabular}




\begin{tabular}{llll}
87. & 169 & End monitoring & \\
88. & 42 & 18 To column & 8.0 \\
89. & 2 & Reverse flush & 5.0 \\
90. & 1 & Block flush & 4.0 \\
91. & 107 & End & \\
\hline
\end{tabular}

Note: Bottle 5-polymerizable capping phosphoramidite; bottle 14—detritylation solution; bottle 15—oxidation solution; bottle 18-acetonitrile; bottle 19dichloromethane; B—base or phosphoramidite; Tet—tetrazole or activator.

Table A.4. Cycle $2-0.2 \mu \mathrm{mol}$ synthesis, the general ODN synthesis procedure with shortened capping time

ODN synthetic cycle using polymerizable phosphoramidite as the capping agent Synthesizer: standard ABI 394 solid phase synthesizer; 4-column 8-base instrument Polymerizable capping agent: 0.15 or $0.1 \mathrm{M}$ solution of polymerizable phosphoramidite in acetonitrile, placed at the bottle 5 position

The bottles for normal $\mathrm{Ac}_{2} \mathrm{O}$ capping agents are empty

Activator for the capping phosphoramidite: from the same bottle for the coupling step Synthesis scale: $0.2 \mu \mathrm{mol}$

Column used: column 2; functions for other columns are not shown

\begin{tabular}{llll}
\hline Step number & Function number & Function name & Step time \\
\hline 1. & 106 & Begin & \\
2. & 64 & 18 To waste & 3.0 \\
3. & 42 & 18 To column & 10.0 \\
4. & 2 & Reverse flush & 8.0 \\
5. & 1 & Block flush & 4.0 \\
6. & 101 & Phos Prep & 3.0 \\
7. & 142 & Column 2 on &
\end{tabular}




\begin{tabular}{|c|c|c|c|}
\hline 8. & 111 & Block vent & 2.0 \\
\hline 9. & 58 & Tet to waste & 1.7 \\
\hline 10. & 33 & B+Tet to column & 2.0 \\
\hline 11. & 34 & Tet to column & 1.0 \\
\hline 12. & 33 & $\mathrm{~B}+\mathrm{Tet}$ to column & 1.5 \\
\hline 13. & 43 & Push to column & \\
\hline 14. & 143 & Column 2 off & \\
\hline 15. & 103 & wait & 25.0 \\
\hline 16. & 64 & 18 To waste & 3.0 \\
\hline 17. & 42 & 18 To column & 10.0 \\
\hline 18. & 2 & Reverse flush & 8.0 \\
\hline 19. & 1 & Block flush & 4.0 \\
\hline 20. & 101 & Phos Prep & 3.0 \\
\hline 21. & 142 & Column 2 on & \\
\hline 22. & 111 & Block vent & 2.0 \\
\hline 23. & 58 & Tet to waste & 1.7 \\
\hline 24. & 35 & $5+$ Tet to column & 2.0 \\
\hline 25. & 34 & Tet to column & 1.0 \\
\hline 26. & 103 & Wait & 30.0 \\
\hline 27. & 35 & $5+$ Tet to column & 1.5 \\
\hline 28. & 103 & Wait & 30.0 \\
\hline 29. & 35 & $5+$ Tet to column & 1.5 \\
\hline 30. & 103 & Wait & 30.0 \\
\hline 31. & 43 & Push to column & \\
\hline 32. & 103 & Wait & 30.0 \\
\hline 33. & 64 & 18 To waste & 4.0 \\
\hline 34. & 42 & 18 To column & 8.0 \\
\hline 35. & 4 & Flush to waste & 4.0 \\
\hline 36. & 42 & 18 To column & 8.0 \\
\hline 37. & 2 & Reverse flush & 5.0 \\
\hline 38. & 42 & 18 To column & 8.0 \\
\hline 39. & 2 & Reverse flush & 5.0 \\
\hline 40. & 1 & Block flush & 3.0 \\
\hline 41. & 41 & 15 To column & 8.0 \\
\hline
\end{tabular}




\begin{tabular}{|c|c|c|c|}
\hline 42. & 64 & 18 To waste & 4.0 \\
\hline 43. & 1 & Block flush & 3.0 \\
\hline 44. & 103 & Wait & 10.0 \\
\hline 45. & 41 & 15 To column & 8.0 \\
\hline 46. & 64 & 18 To waste & 4.0 \\
\hline 47. & 1 & Block flush & 3.0 \\
\hline 48. & 103 & Wait & 10.0 \\
\hline 49. & 42 & 18 To column & 15.0 \\
\hline 50. & 4 & Flush to waste & 4.0 \\
\hline 51. & 42 & 18 To column & 10.0 \\
\hline 52. & 2 & Reverse flush & 5.0 \\
\hline 53. & 42 & 18 To column & 10.0 \\
\hline 54. & 2 & Reverse flush & 5.0 \\
\hline 55. & 1 & Block flush & 3.0 \\
\hline 56. & 105 & Start detrityl & \\
\hline 57. & 64 & 18 To waste & 4.0 \\
\hline 58. & 42 & 18 To column & 10.0 \\
\hline 59. & 2 & Reverse flush & 5.0 \\
\hline 60. & 1 & Block flush & 3.0 \\
\hline 61. & 167 & If monitoring & \\
\hline 62. & 44 & 19 To column & 25.0 \\
\hline 63. & 40 & 14 To column & 3.0 \\
\hline 64. & 135 & Monitor triyls & \\
\hline 65. & 40 & 14 To column & 25.0 \\
\hline 66. & 136 & Monitor noise & \\
\hline 67. & 40 & 14 To column & 10.0 \\
\hline 68. & 137 & Stop monitor & \\
\hline 69. & 42 & 18 To column & 10.0 \\
\hline 70. & 2 & Reverse flush & 8.0 \\
\hline 71. & 168 & If not monitoring & \\
\hline 72. & 40 & 14 To column & 6.0 \\
\hline 73. & 3 & Trityl flush & 5.0 \\
\hline 74. & 40 & 14 To column & 6.0 \\
\hline 75. & 103 & Wait & 5.0 \\
\hline
\end{tabular}




\begin{tabular}{llll}
76. & 3 & Trityl flush & 5.0 \\
77. & 40 & 14 To column & 6.0 \\
78. & 103 & Wait & 5.0 \\
79. & 3 & Trityl flush & 5.0 \\
80. & 40 & 14 To column & 6.0 \\
81. & 103 & Wait & 5.0 \\
82. & 3 & Trityl flush & 5.0 \\
83. & 42 & 18 To column & 10.0 \\
84. & 3 & Trityl flush & 8.0 \\
85. & 169 & End monitoring & \\
86. & 42 & 18 To column & 8.0 \\
87. & 2 & Reverse flush & 5.0 \\
88. & 1 & Block flush & 4.0 \\
89. & 107 & End & \\
\hline
\end{tabular}

Note: Bottle 5-polymerizable capping phosphoramidite; bottle 14—detritylation solution; bottle 15—oxidation solution; bottle 18-acetonitrile; bottle 19dichloromethane; B—base or phosphoramidite; Tet—tetrazole or activator.

Table A.5. Cycle $3-1.0 \mu \mathrm{mol}$ synthesis

ODN synthetic cycle using polymerizable phosphoramidite as the capping agent Synthesizer: standard ABI 394 solid phase synthesizer; 4-column 8-base instrument Polymerizable capping agent: $0.15 \mathrm{M}$ solution of polymerizable phosphoramidite in acetonitrile unless otherwise noted in the article, placed at the bottle 5 position The bottles for normal $\mathrm{Ac}_{2} \mathrm{O}$ capping agents are empty

Activator for the capping phosphoramidite: from the same bottle for the coupling step Synthesis scale: $1.0 \mu \mathrm{mol}$

Column used: column 2; functions for other columns are not shown

\begin{tabular}{llll}
\hline Step number & Function number & Function name & Step time \\
\hline 1. & 106 & Begin & \\
2. & 64 & 18 To waste & 3.0 \\
3. & 42 & 18 To column & 10.0 \\
4. & 2 & Reverse flush & 10.0 \\
5. & 1 & Block flush & 4.0 \\
6. & 101 & Phos Prep & 3.0 \\
& & 220 &
\end{tabular}




\begin{tabular}{|c|c|c|c|}
\hline 7. & 142 & Column 2 on & \\
\hline 8. & 111 & Block vent & 2.0 \\
\hline 9. & 58 & Tet to waste & 1.7 \\
\hline 10. & 33 & $\mathrm{~B}+\mathrm{Tet}$ to column & 2.5 \\
\hline 11. & 34 & Tet to column & 1.0 \\
\hline 12. & 33 & $\mathrm{~B}+\mathrm{Tet}$ to column & 2.5 \\
\hline 13. & 43 & Push to column & \\
\hline 14. & 143 & Column 2 off & \\
\hline 15. & 103 & wait & 50.0 \\
\hline 16. & 64 & 18 To waste & 3.0 \\
\hline 17. & 42 & 18 To column & 10.0 \\
\hline 18. & 4 & Flush to waste & 5.0 \\
\hline 19. & 42 & 18 To column & 10.0 \\
\hline 20. & 2 & Reverse flush & 10.0 \\
\hline 21. & 1 & Block flush & 4.0 \\
\hline 22. & 101 & Phos Prep & 3.0 \\
\hline 23. & 142 & Column 2 on & \\
\hline 24. & 111 & Block vent & 2.0 \\
\hline 25. & 58 & Tet to waste & 1.7 \\
\hline 26. & 35 & $5+$ Tet to column & 2.5 \\
\hline 27. & 34 & Tet to column & 1.0 \\
\hline 28. & 103 & Wait & 30.0 \\
\hline 29. & 35 & $5+$ Tet to column & 2.5 \\
\hline 30. & 103 & Wait & 30.0 \\
\hline 31. & 35 & $5+$ Tet to column & 2.5 \\
\hline 32. & 103 & Wait & 30.0 \\
\hline 33. & 43 & Push to column & \\
\hline 34. & 103 & Wait & 30.0 \\
\hline 35. & 64 & 18 To waste & 4.0 \\
\hline 36. & 42 & 18 To column & 10.0 \\
\hline 37. & 4 & Flush to waste & 6.0 \\
\hline 38. & 42 & 18 To column & 10.0 \\
\hline 39. & 4 & Flush to waste & 6.0 \\
\hline 40. & 42 & 18 To column & 10.0 \\
\hline
\end{tabular}




\begin{tabular}{|c|c|c|c|}
\hline 41. & 2 & Reverse flush & 7.0 \\
\hline 42. & 1 & Block flush & 3.0 \\
\hline 43. & 41 & 15 To column & 8.0 \\
\hline 44. & 64 & 18 To waste & 4.0 \\
\hline 45. & 1 & Block flush & 3.0 \\
\hline 46. & 103 & Wait & 15.0 \\
\hline 47. & 41 & 15 To column & 8.0 \\
\hline 48. & 64 & 18 To waste & 4.0 \\
\hline 49. & 1 & Block flush & 3.0 \\
\hline 50. & 103 & Wait & 15.0 \\
\hline 51. & 42 & 18 To column & 15.0 \\
\hline 52. & 4 & Flush to waste & 6.0 \\
\hline 53. & 42 & 18 To column & 10.0 \\
\hline 54. & 4 & Flush to waste & 6.0 \\
\hline 55. & 42 & 18 To column & 10.0 \\
\hline 56. & 2 & Reverse flush & 7.0 \\
\hline 57. & 1 & Block flush & 3.0 \\
\hline 58. & 105 & Start detrityl & \\
\hline 59. & 64 & 18 To waste & 4.0 \\
\hline 60. & 42 & 18 To column & 10.0 \\
\hline 61. & 2 & Reverse flush & 5.0 \\
\hline 62. & 1 & Block flush & 3.0 \\
\hline 63. & 167 & If monitoring & \\
\hline 64. & 44 & 19 To column & 25.0 \\
\hline 65. & 40 & 14 To column & 3.0 \\
\hline 66. & 135 & Monitor triyls & \\
\hline 67. & 40 & 14 To column & 25.0 \\
\hline 68. & 136 & Monitor noise & \\
\hline 69. & 40 & 14 To column & 10.0 \\
\hline 70. & 137 & Stop monitor & \\
\hline 71. & 42 & 18 To column & 10.0 \\
\hline 72. & 2 & Reverse flush & 8.0 \\
\hline 73. & 168 & If not monitoring & \\
\hline \multirow[t]{2}{*}{74.} & 40 & 14 To column & 6.0 \\
\hline & & 222 & \\
\hline
\end{tabular}




\begin{tabular}{llll}
75. & 3 & Trityl flush & 5.0 \\
76. & 40 & 14 To column & 6.0 \\
77. & 103 & Wait & 5.0 \\
78. & 3 & Trityl flush & 5.0 \\
79. & 40 & 14 To column & 6.0 \\
80. & 103 & Wait & 5.0 \\
81. & 3 & Trityl flush & 5.0 \\
82. & 40 & 14 To column & 6.0 \\
83. & 103 & Wait & 5.0 \\
84. & 3 & Trityl flush & 5.0 \\
85. & 42 & 18 To column & 10.0 \\
86. & 3 & Trityl flush & 8.0 \\
87. & 169 & End monitoring & \\
88. & 42 & 18 To column & 8.0 \\
89. & 2 & Reverse flush & 5.0 \\
90. & 1 & Block flush & 4.0 \\
91. & 107 & End & \\
\hline
\end{tabular}

Note: Bottle 5-polymerizable capping phosphoramidite; bottle 14-detritylation solution; bottle 15-oxidation solution; bottle 18 - acetonitrile; bottle 19dichloromethane; B—base or phosphoramidite; Tet—-tetrazole or other activator. 


\section{Appendix B}

Supporting Information for Chapter 3

A Highly Convenient Procedure for Oligodeoxynucleotide Purification 


\section{Recipes for Preparation of Polymerization Solutions}

$3.7 \mathrm{M}$ of $N, N$-Dimethylacrylamide and $0.37 \mathrm{M} \quad N, N^{\prime}$-methylenebis(acrylamide)

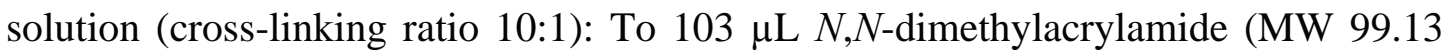
$\mathrm{mg} / \mathrm{mmol}$, density $962 \mu \mathrm{g} / \mu \mathrm{L}, 100 \mathrm{mg}, \quad 1 \mathrm{mmol})$ and $15.5 \mathrm{mg} N, N^{\prime}-$ methylenebis(acrylamide) (MW $154.17 \mathrm{mg} / \mathrm{mmol}, 0.1 \mathrm{mmol}$ ), add $103 \mu \mathrm{L}$ water. Mix to dissolve. The final volume of the solutions is $272 \mu \mathrm{L}$.

$5 \%\left(\mathrm{NH}_{4}\right)_{2} \mathrm{~S}_{2} \mathrm{O}_{8}$ Solution: To $5 \mathrm{mg}\left(\mathrm{NH}_{4}\right)_{2} \mathrm{~S}_{2} \mathrm{O}_{8}(\mathrm{MW} 228.18 \mathrm{mg} / \mathrm{mmol})$, add $95 \mu \mathrm{L}$ water. Mix well $\left(5 \mu \mathrm{L}\right.$ solution contains $\left.1.1 \mu \mathrm{mol}\left(\mathrm{NH}_{4}\right)_{2} \mathrm{~S}_{2} \mathrm{O}_{8}\right)$.

0.66 M TMEDA solution: To $49 \mu \mathrm{L}$ TMEDA (MW $116.24 \mathrm{mg} / \mathrm{mmol}$, density 0.777

$\mathrm{mg} / \mu \mathrm{L}, 38.1 \mathrm{mg}, 0.33 \mathrm{mmol}$ ), add water until the total volume reaches $500 \mu \mathrm{L}$. Mix well (5 $\mu \mathrm{L}$ solution contains $3.3 \mu \mathrm{mol}$ TMEDA $)$.

\section{HPLC conditions}

Column: C-18, $5 \mu \mathrm{m}, 100 \AA$ A $250 \times 3.20 \mathrm{~mm}$; solvent A: $0.1 \mathrm{M}$ triethylammonium acetate, 5\% acetonitrile; solvent B: $90 \%$ acetonitrile; flow rate: $0.5 \mathrm{~mL} / \mathrm{min}$; detection: $260 \mathrm{~nm}$; faster gradient: solvent B (0\%-45\%) in solvent A over $60 \mathrm{~min}$; slower gradient: solvent B (0\%-15\%) in solvent A over 60 min. 


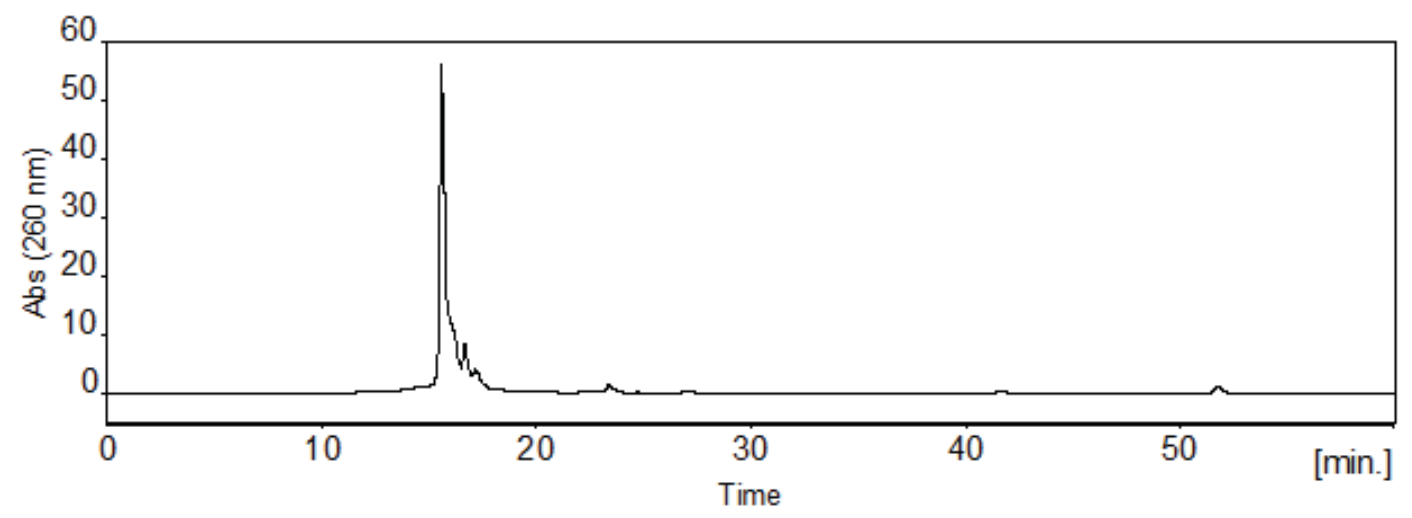

Figure B.1. HPLC of crude ODN 3.2, faster gradient. The enlarged version of the profile shown in trace a in Figure 3.2. Failure sequences were capped with polymerizable phosphoramidite during synthesis, and thus have longer retention times than the full-length sequence. ODN 3.2 is 5'-TCA TTG CTG CTT AGA CCG CT-3'.

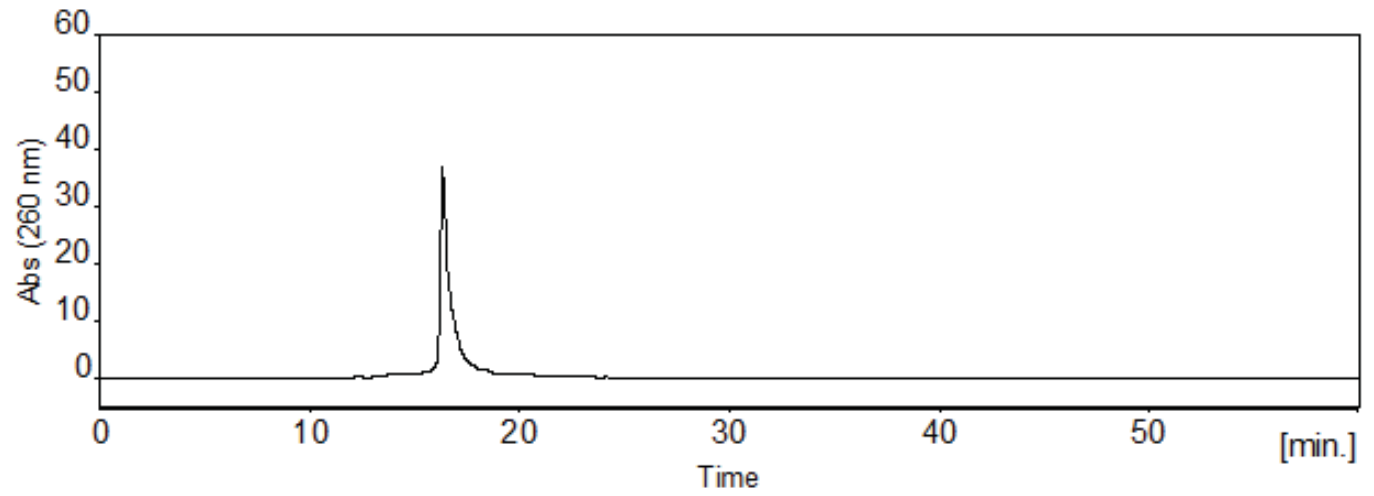

Figure B.2. HPLC of pure ODN 3.2, faster gradient. The enlarged version of the profile shown in trace $\mathrm{b}$ in Figure 3.2. The ODN was purified by catching failure sequences by polymerization. 


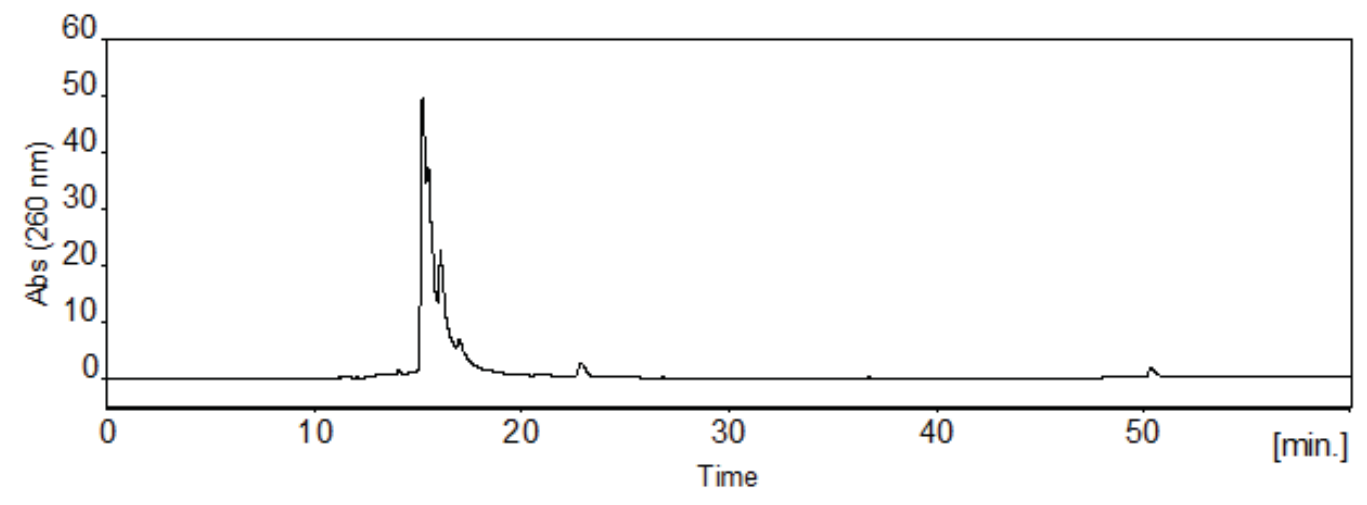

Figure B.3. HPLC of crude 19-mer ODN 3.3, faster gradient. The 19-mer ODN 3 (5'CAT TGC TGC TTA GAC CGC T-3'), which is the same as $\mathbf{3 . 2}$ except that there is one less base at the 5'-end, was synthesized using the same procedure for 3.2.

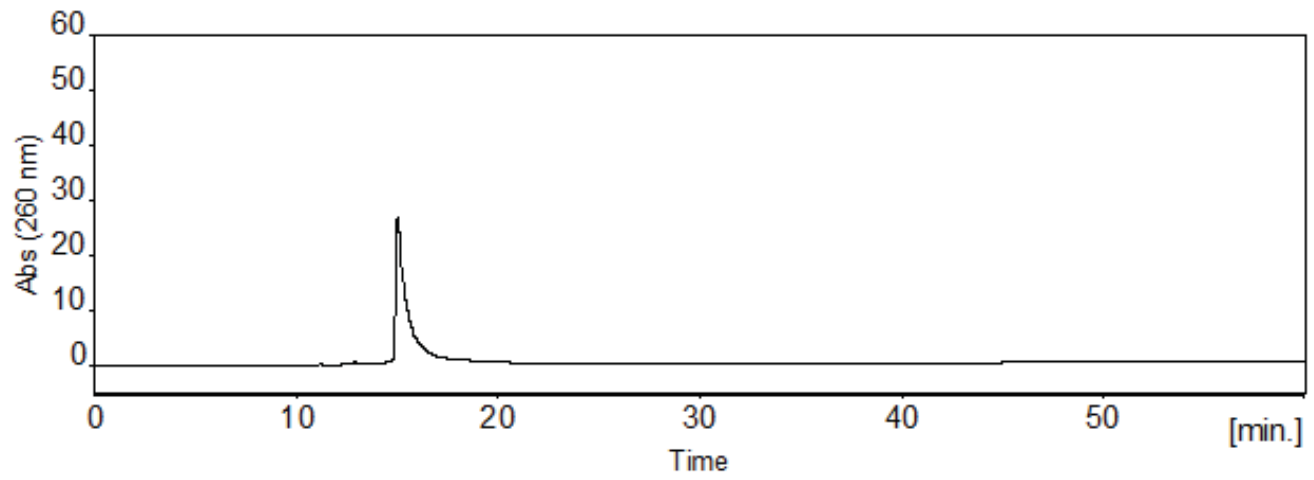

Figure B.4. HPLC of pure 19-mer ODN 3.3, faster gradient. The 19-mer ODN 3.3 (5'-CAT TGC TGC TTA GAC CGC T-3') was purified using the same procedure for 3.2. 


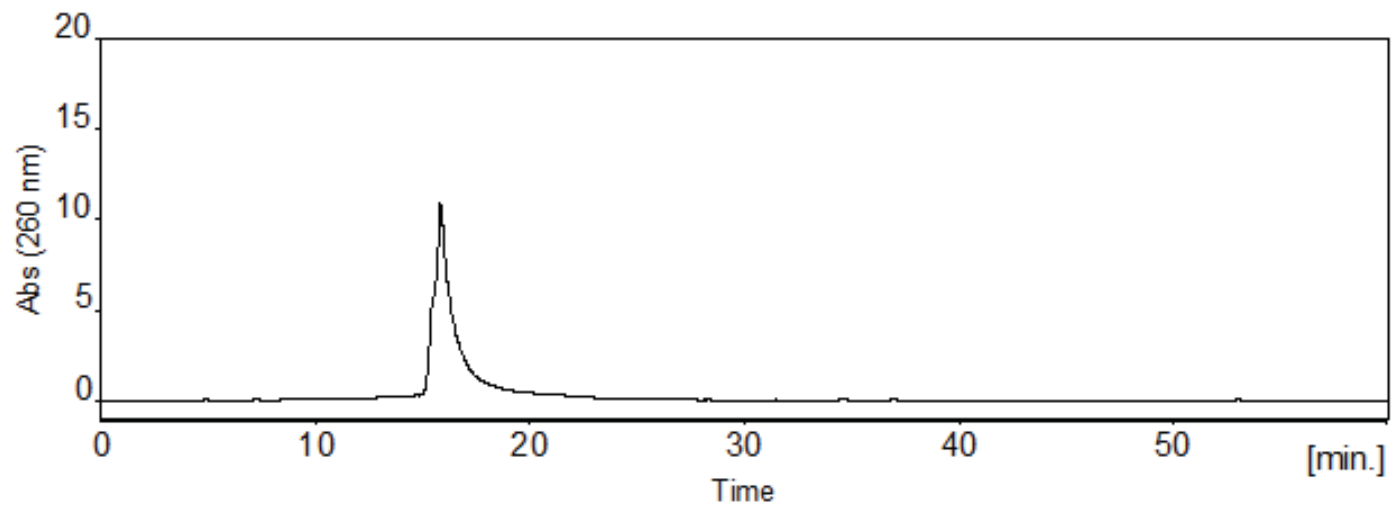

Figure B.5. HPLC of ODNs $\mathbf{3 . 2}$ and 3.3, faster gradient. ODN 3.2 and 3.3 were coinjected into HPLC. A broadened peak was observed indicating that HPLC can resolve the 19-mer failure sequence from 20-mer full-length sequence.

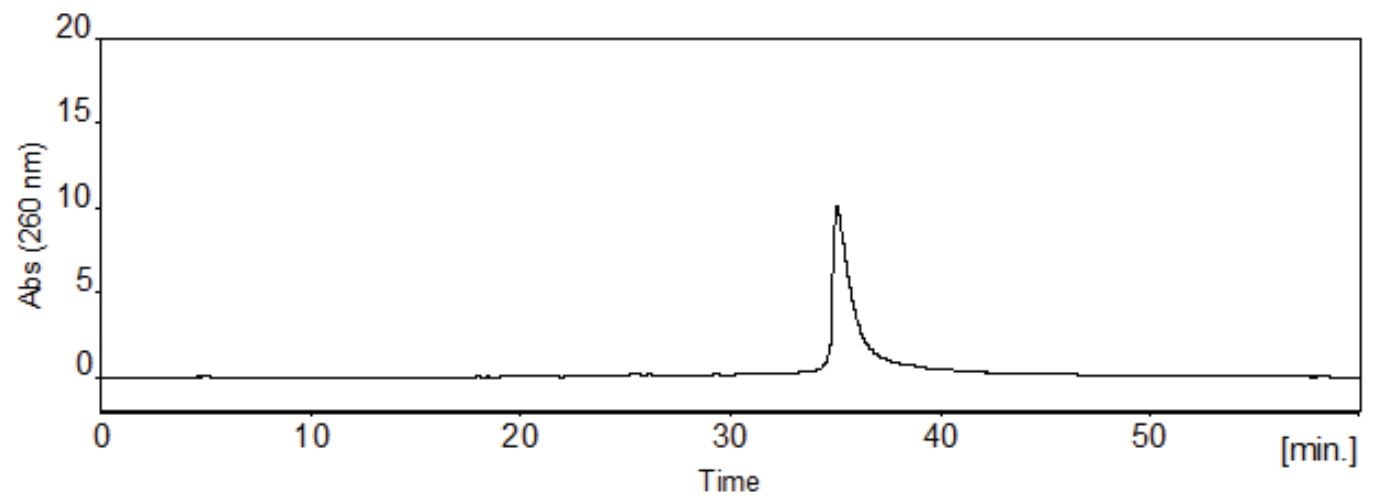

Figure B.6. HPLC of pure ODN 3.2, slower gradient 


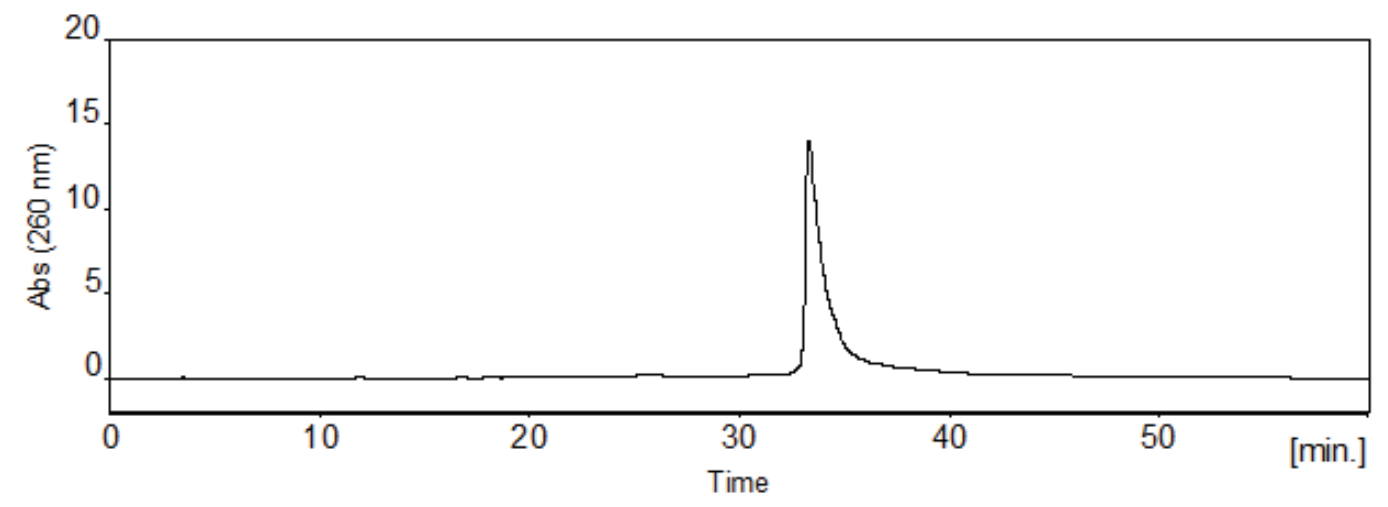

Figure B.7. HPLC of pure 19-mer ODN 3.3, slower gradient

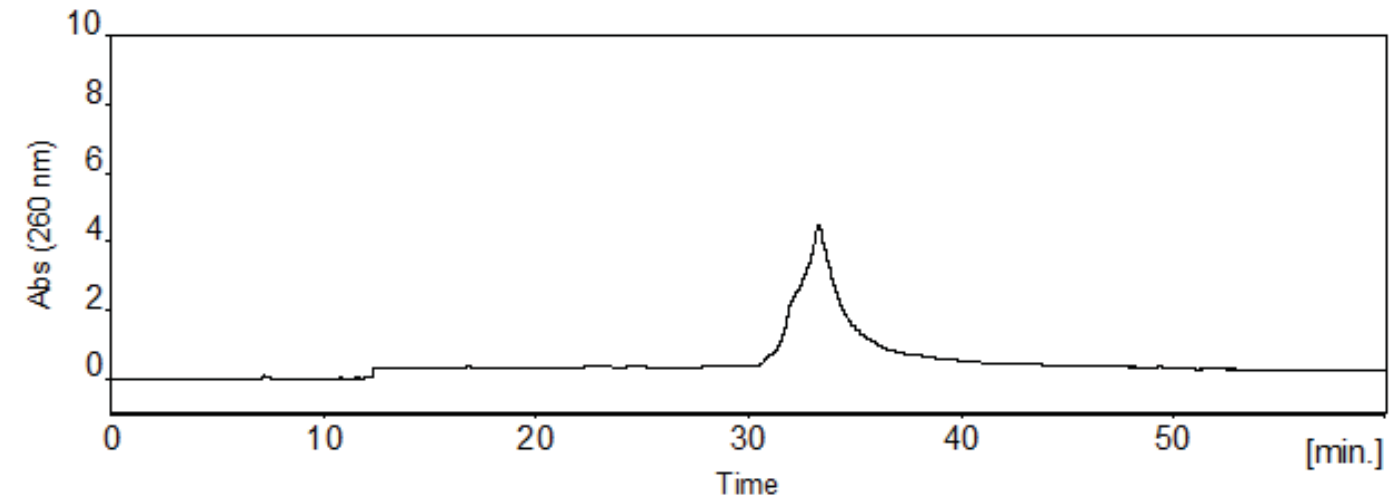

Figure B.8. HPLC of ODNs 3.2 and 3.3, slower gradient ODNs $\mathbf{3 . 2}$ and $\mathbf{3 . 3}(\sim 1: 1$ ratio) were co-injected into HPLC. With slower gradient, the two were better resolved. Under the same conditions, pure ODN 3.2 does not show a shoulder at the left side of the peak. 


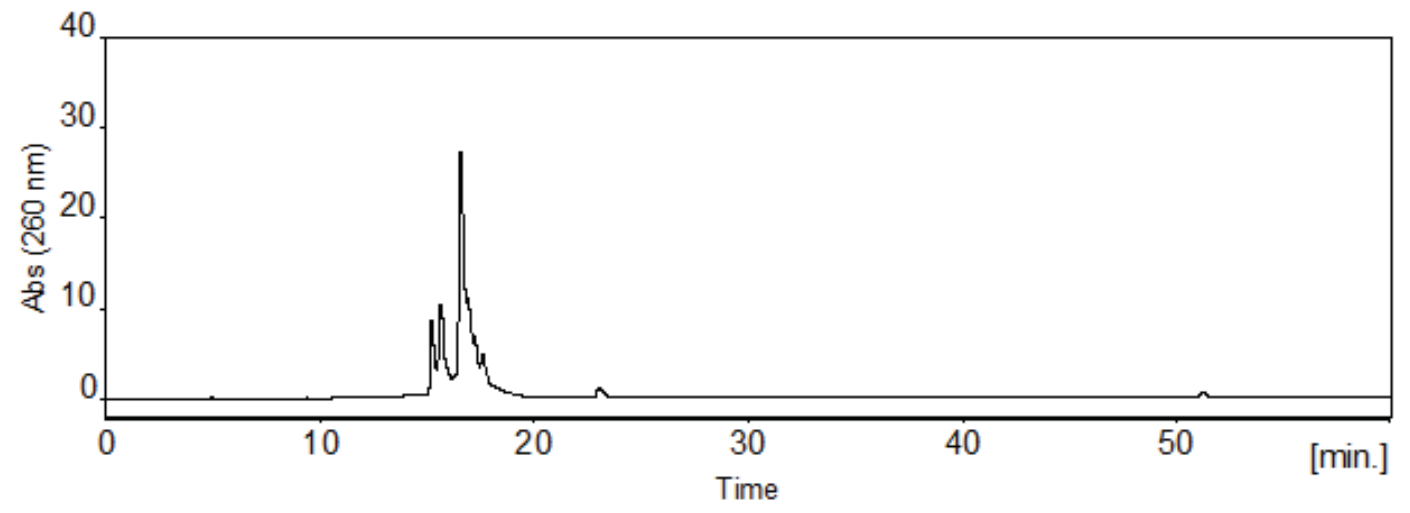

Figure B.9. HPLC of 20-mer ODN 3.4 containing 8-oxo-dG treated with ammonia. The 20-mer ODN (3.4) that contains an 8-oxo-dG [5'-TCA TTG CT(8-oxo-dG) CTT AGA CCG CT-3'] was synthesized and purified with reversed-phase HPLC (RSC $A d v . \mathbf{2 0 1 4}, 4,8746$ ). The product was heated in concentrated $\mathrm{NH}_{4} \mathrm{OH}$ at $80{ }^{\circ} \mathrm{C}$ for 15 min. After cooling to room temperature, $n \mathrm{BuOH}$ was added. The precipitate was analyzed with HPLC. The fast gradient was used. The multiple peaks in the profile indicate that the ODN is not stable under such conditions. Because ODN 3.2 was also processed under the same conditions during purification and only showed a single peak, we infer that 3.2 does not contain damaged dG. This experiment gives additional proof that ODN is stable under radical acrylamide polymerization conditions. 


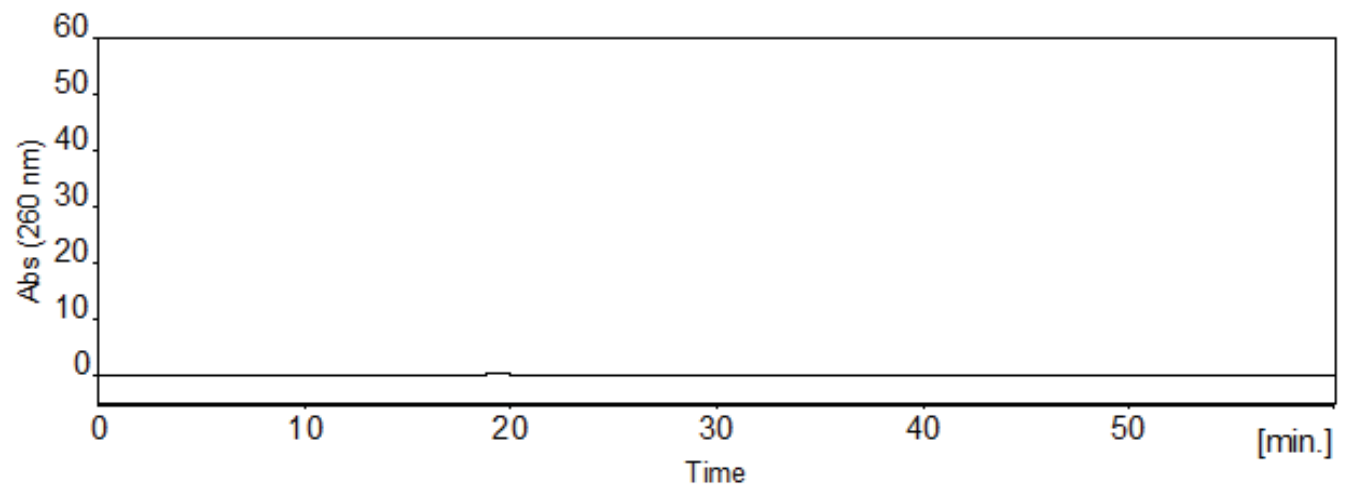

Figure B.10. HPLC of residue from $4^{\text {th }}$ extraction of gel. During purification of ODN 3.2 using the catching by polymerization approach, after extraction of full-length sequence with water for three times, the gel was extracted for an another time. The $4^{\text {th }}$ extract was processed using the same $n \mathrm{BuOH}$ precipitation procedure and analyzed with HPLC. No UV active material is detectable indicating that three extractions are sufficient. 


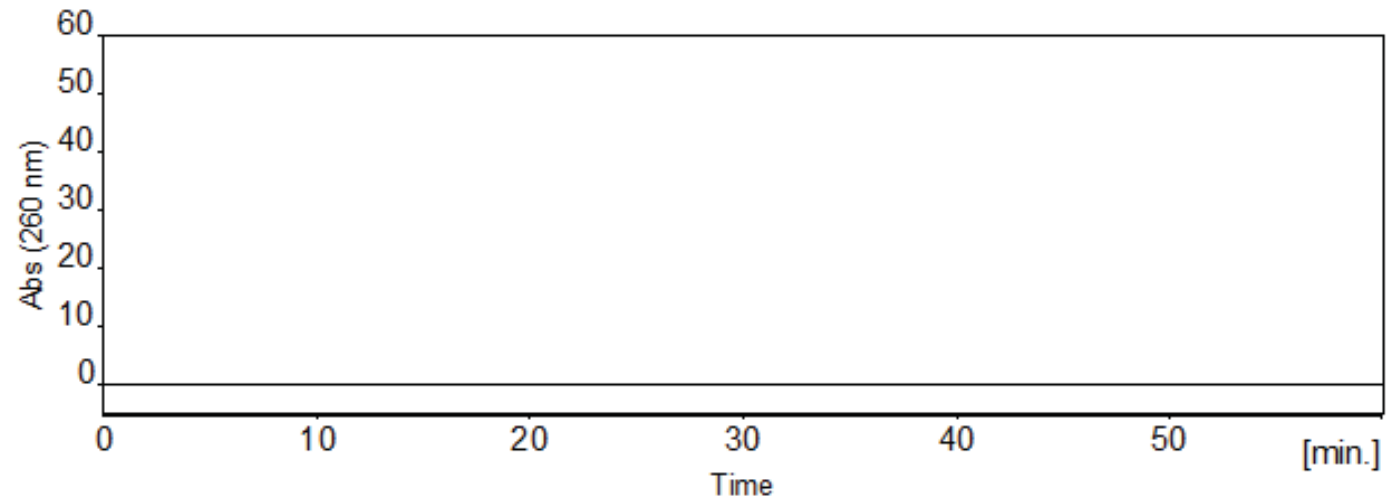

Figure B.11. HPLC of blank control experiment. The catching failure sequences by polymerization ODN purification procedure was followed except that crude ODN was not added. After $n \mathrm{BuOH}$ precipitation and removal of supernatant, no solid residue was observable in the tube. The tube was washed with $30 \mu \mathrm{L}$ water, and 20 $\mu \mathrm{L}$ was injected into HPLC to generate the profile, which showed no UV active material. 


\section{Appendix C}

Supporting Information for Chapter 4

Methacrylation Phosphoramidite with Acid-Cleavable Linker for Eco-

Friendly Synthetic Oligodeoxynucleotide Purification 


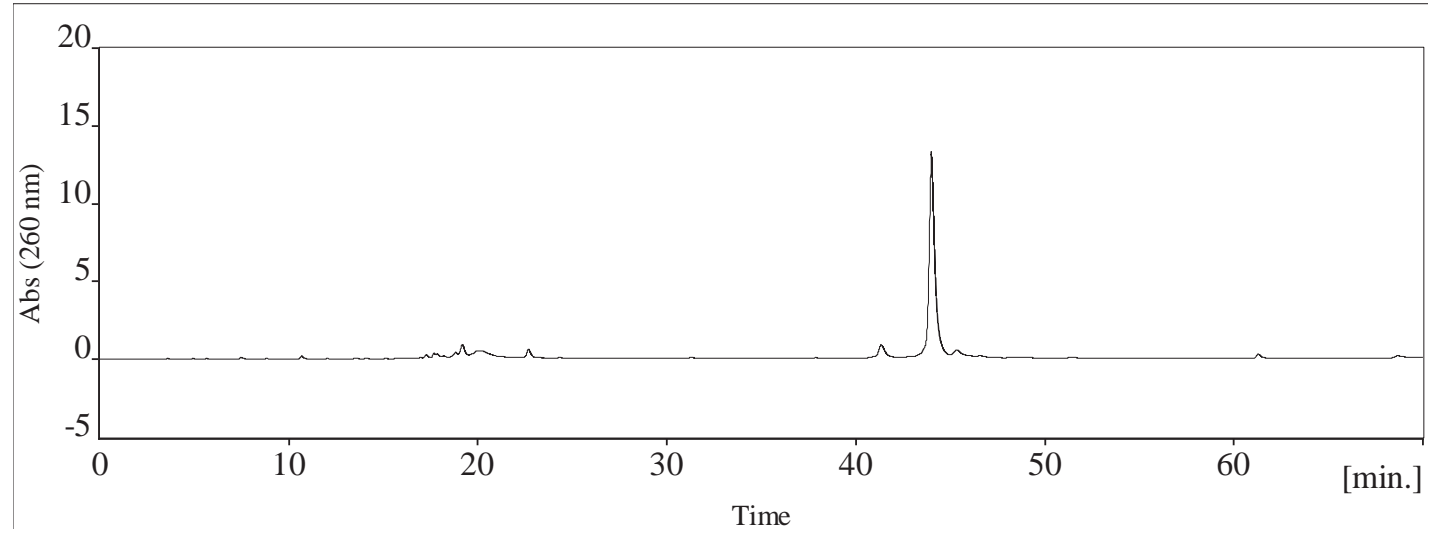

Figure C.1. Crude RP HPLC profile of the 31-mer ODN 4.9

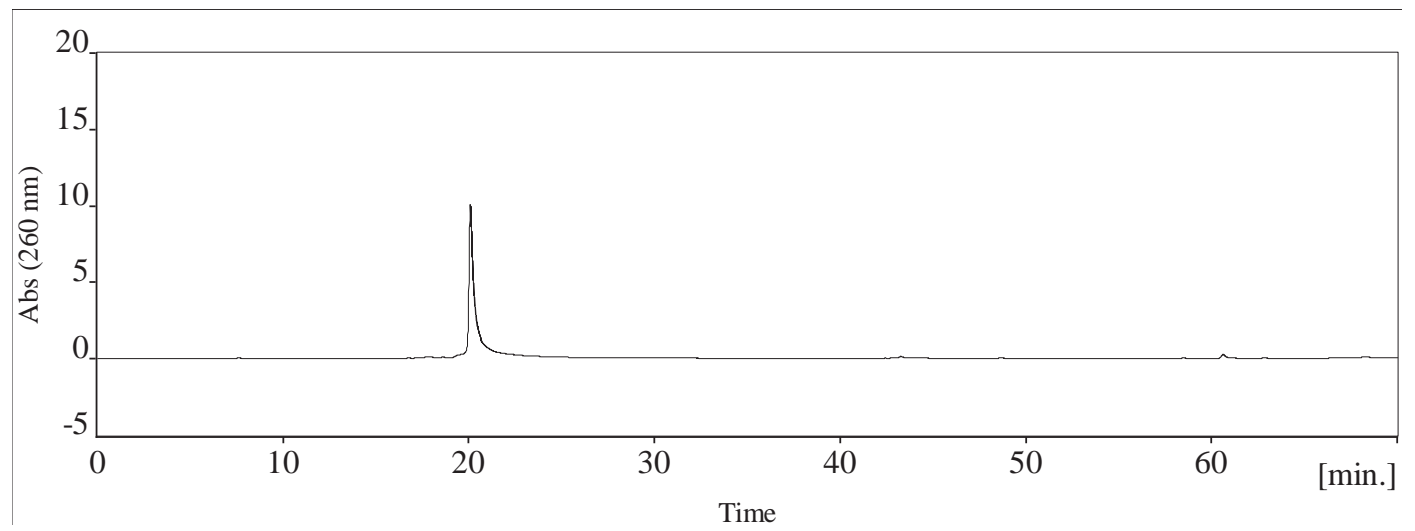

Figure C.2. Pure RP HPLC profile of the 31-mer ODN 4.9 purified on a $0.2 \mu \mathrm{mol}$ scale 


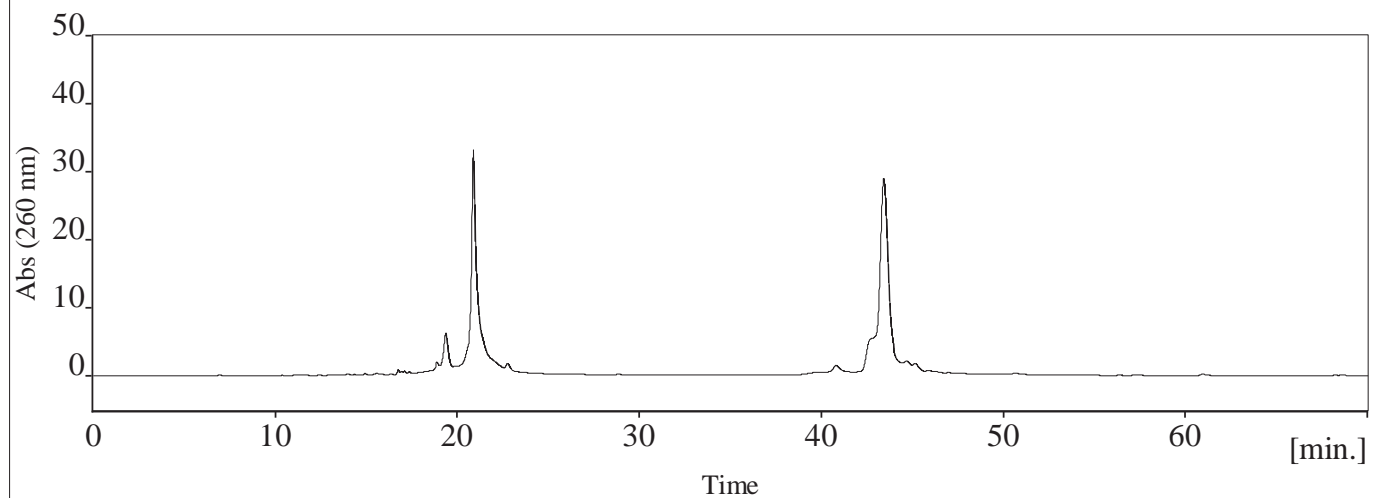

Figure C.3. Crude RP HPLC profile of 37-mer ODN 4.10

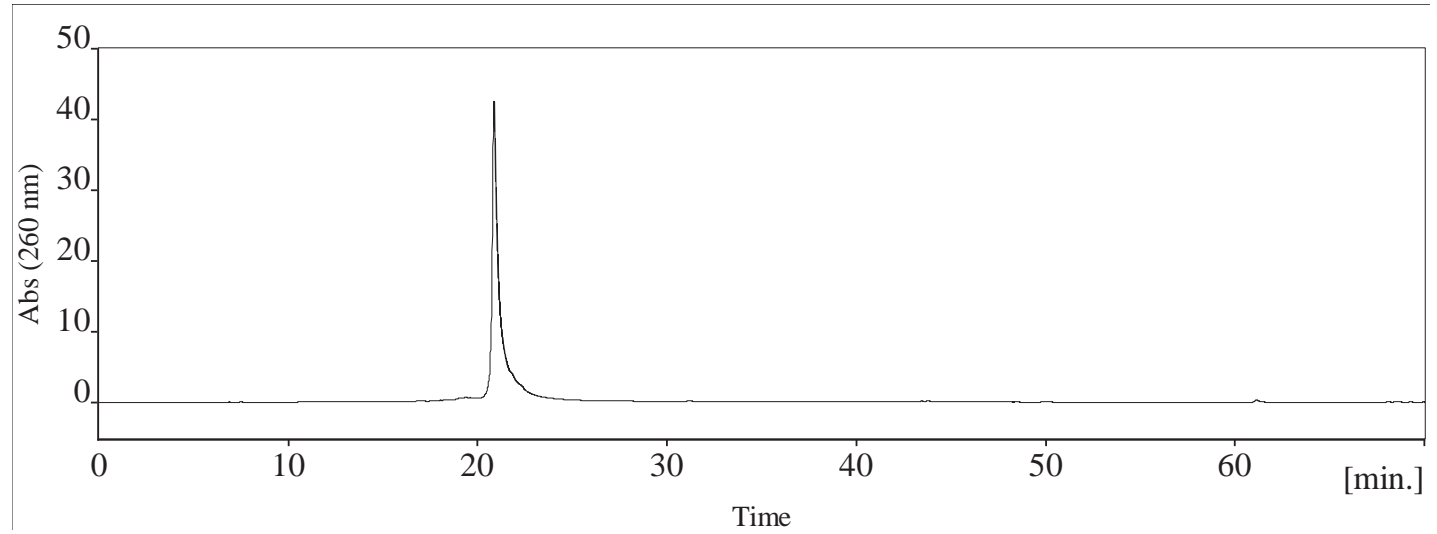

Figure C.4. Pure RP HPLC profile of 37-mer ODN 4.10 purified on a $1 \mu \mathrm{mol}$ scale 


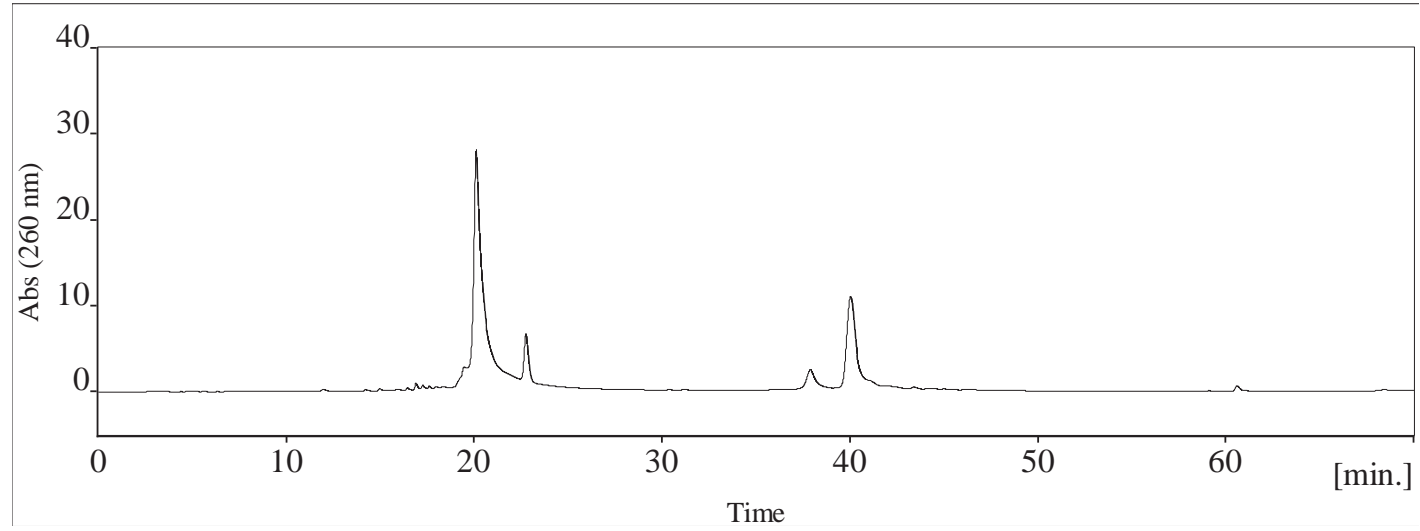

Figure C.5. Crude RP HPLC profile of the 61-mer ODN 4.11

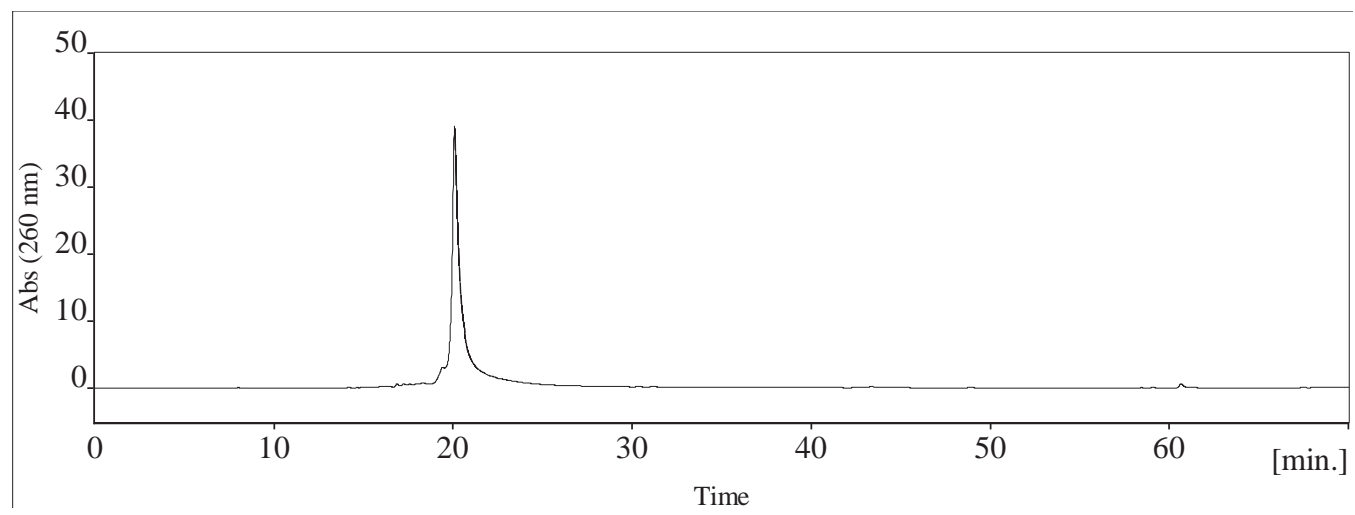

Figure C.6. Pure RP HPLC profile of the 61-mer ODN 4.11 purified on a $0.2 \mu$ mol scale 


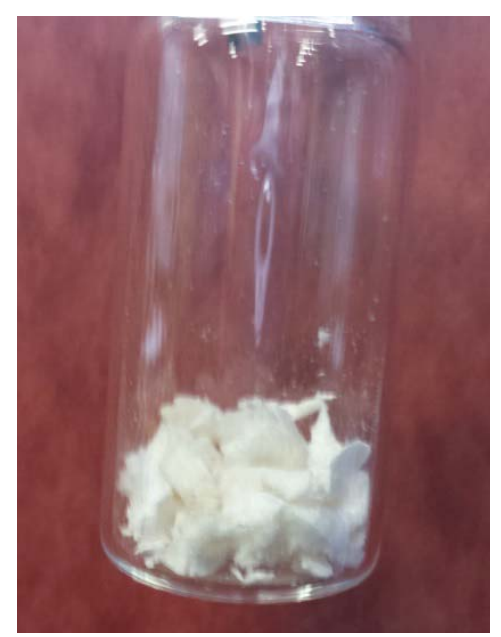

Figure C.7. The 37-mer ODN 4.10 purified with the catching by polymerization technology 


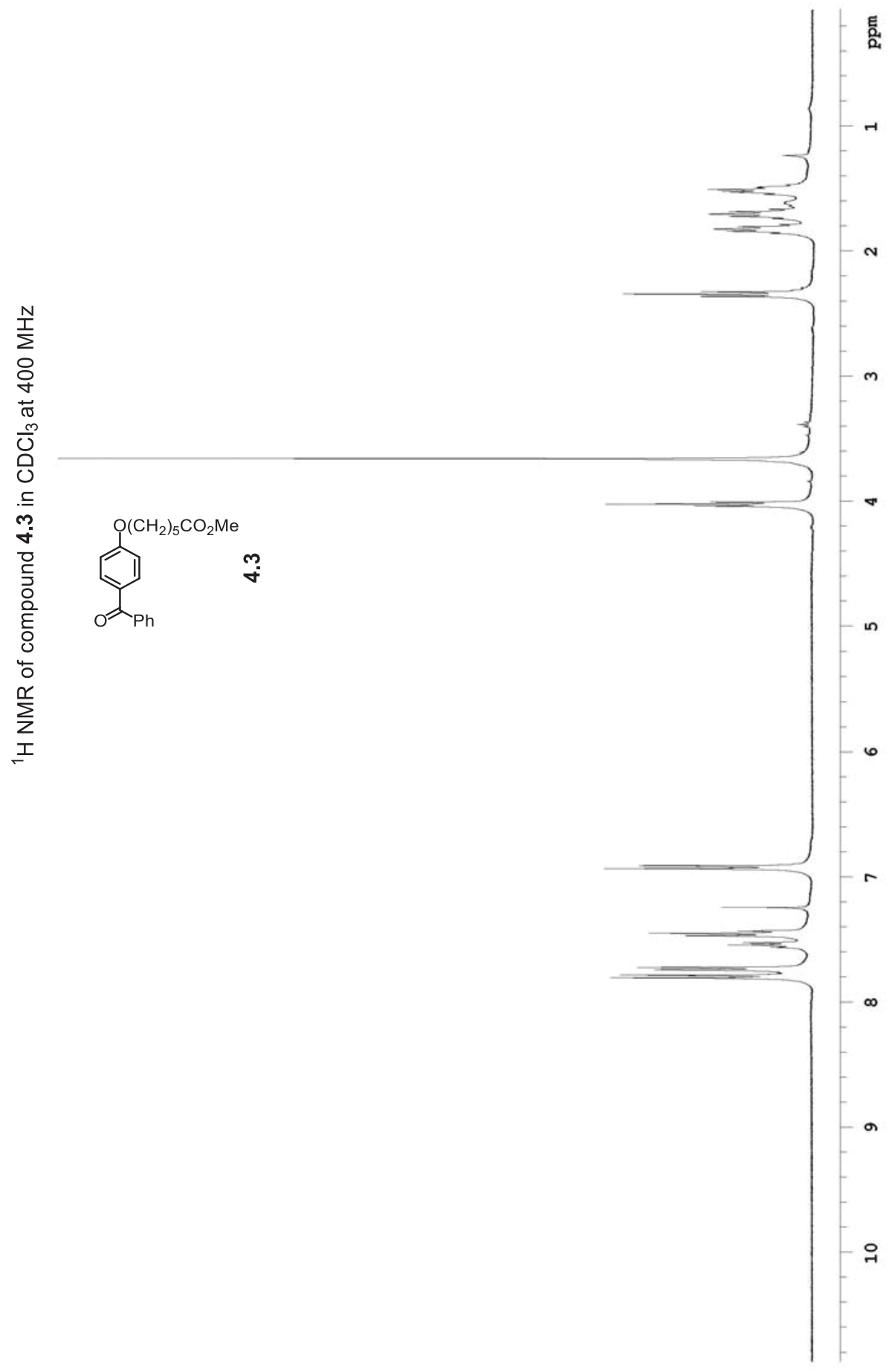

Figure C.8. ${ }^{1} \mathrm{H}$ NMR of compound 4.3 


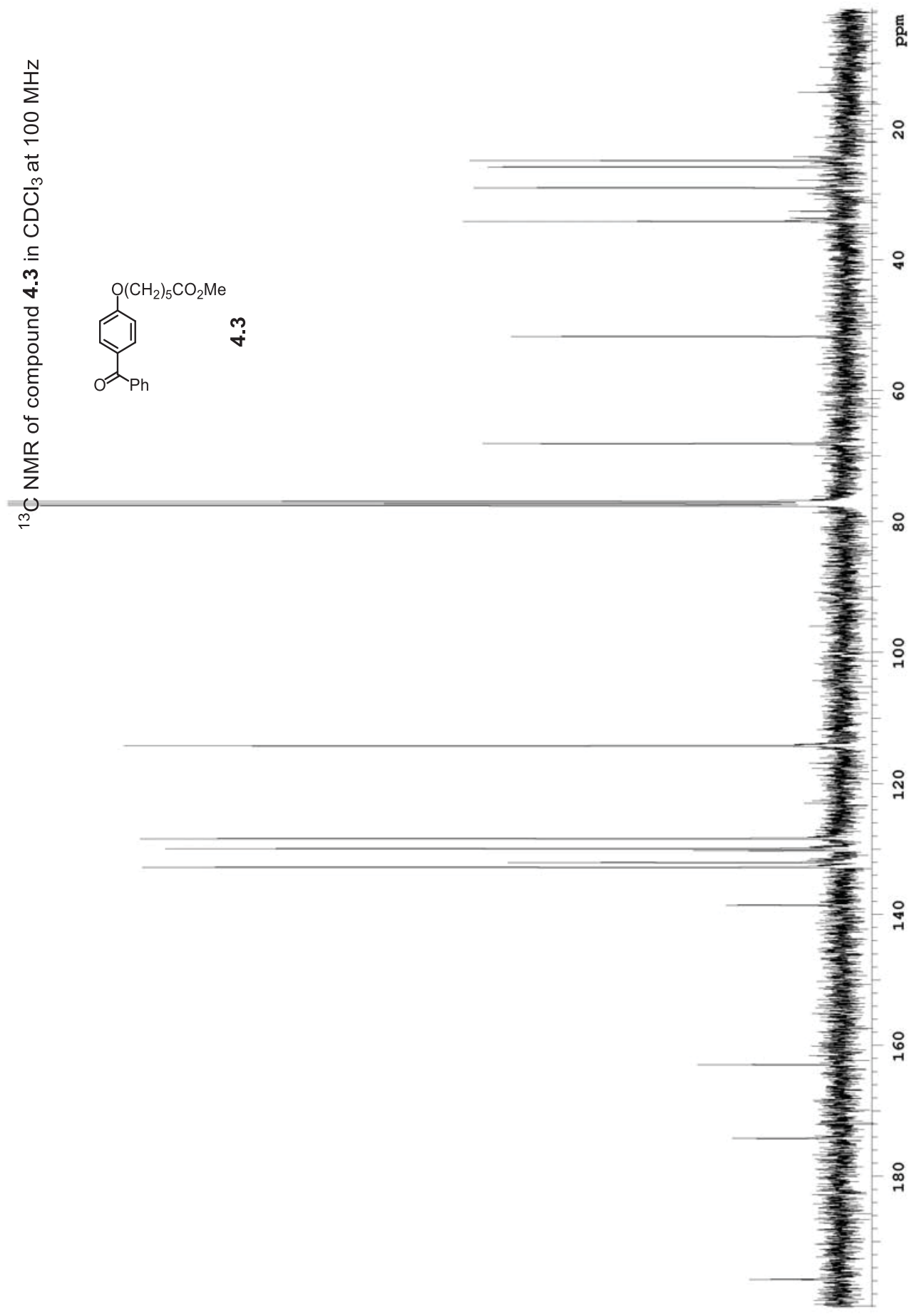

Figure C.9. ${ }^{13} \mathrm{C}$ NMR of compound 4.3 

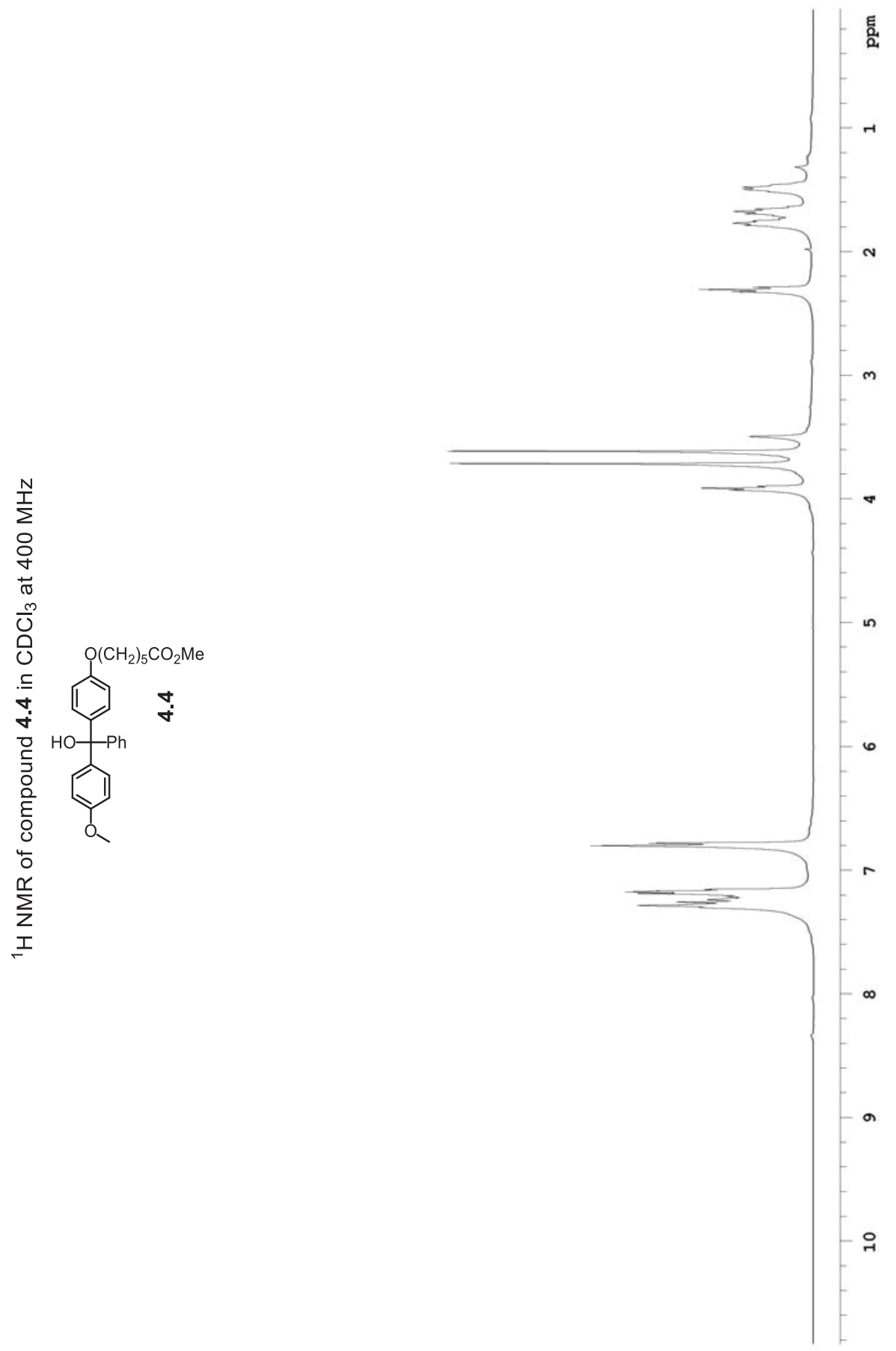

Figure C.10. ${ }^{1} \mathrm{H}$ NMR of compound 4.4 


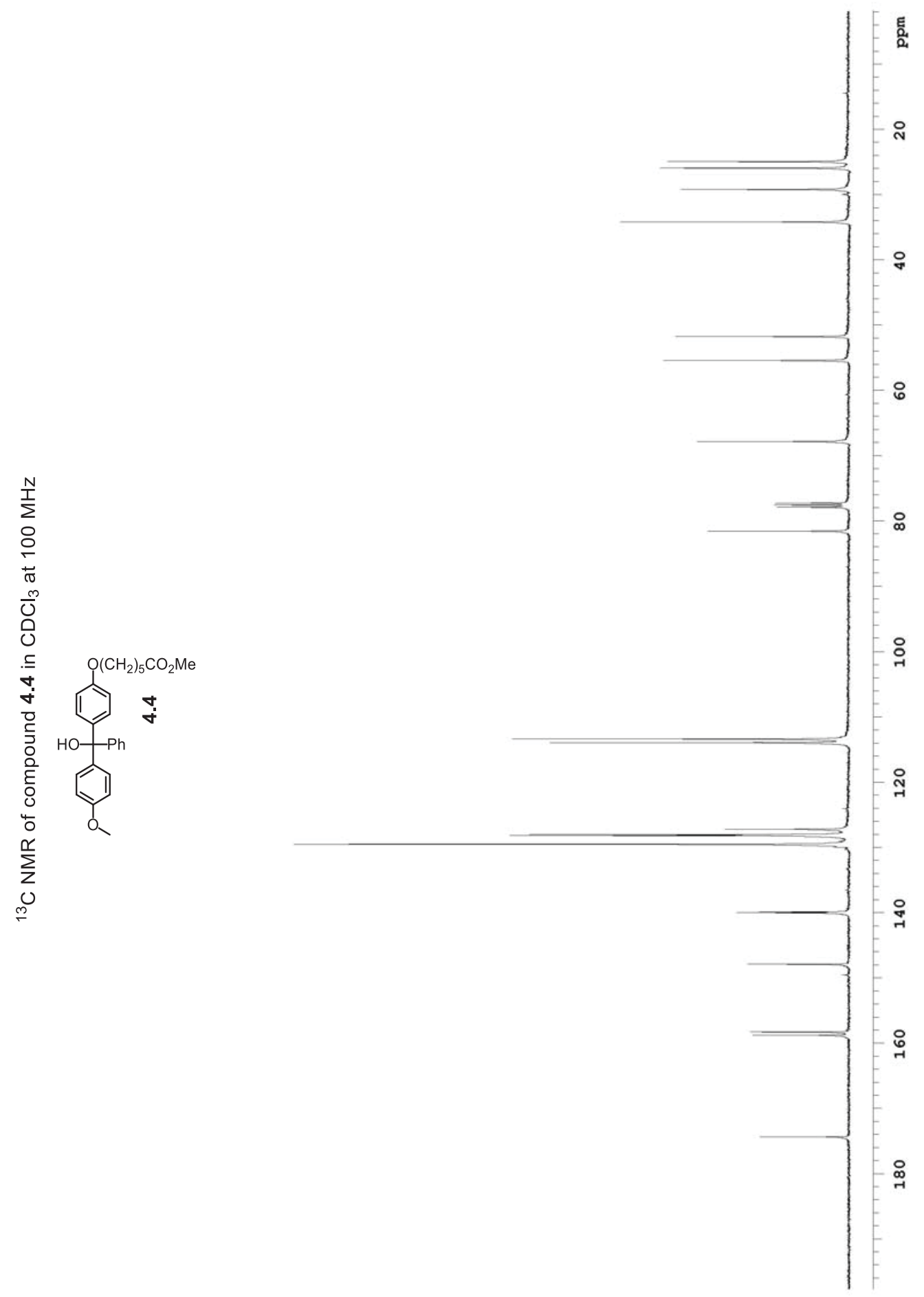

Figure C.11. ${ }^{13} \mathrm{C}$ NMR of compound 4.4 


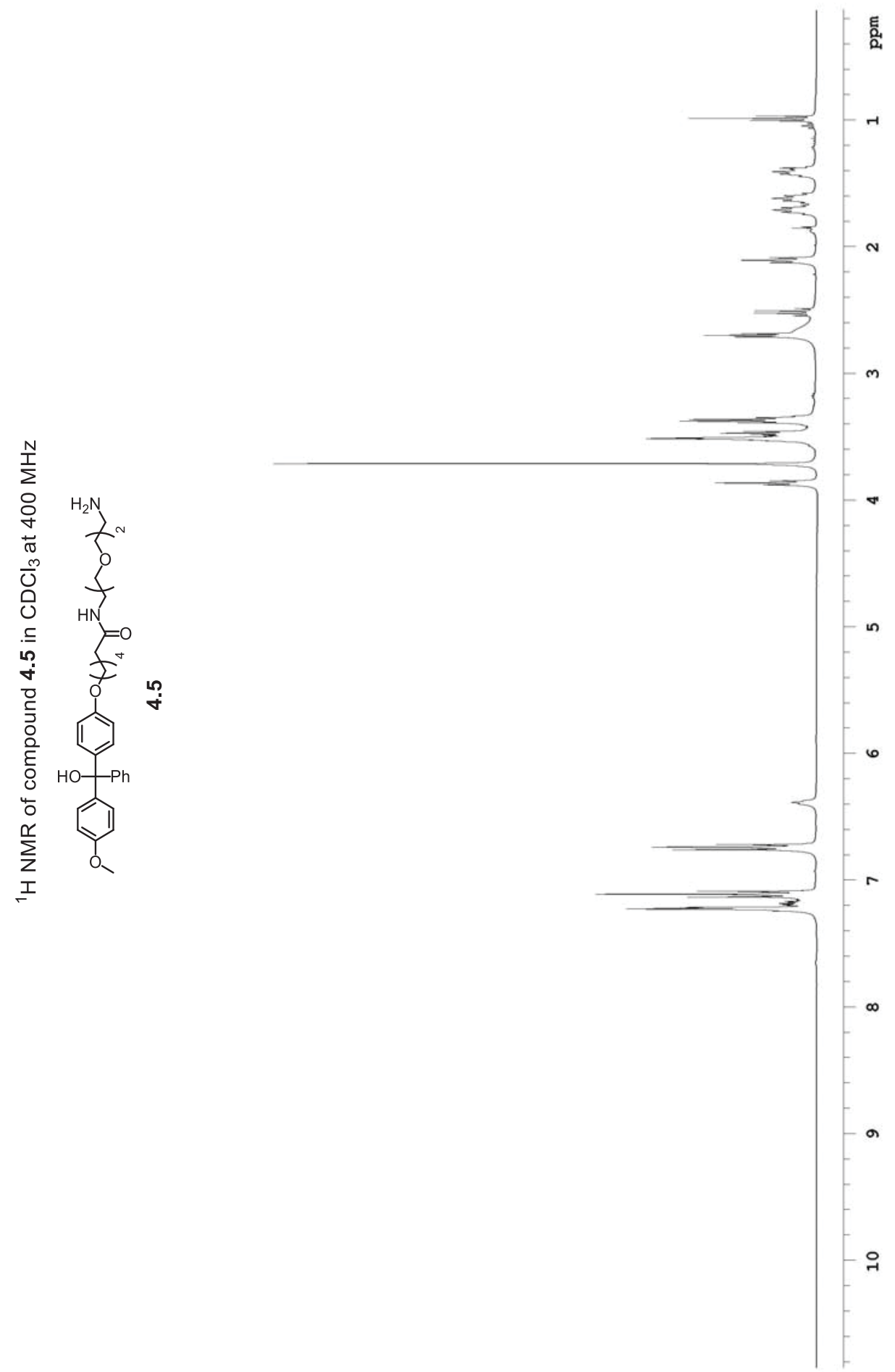

Figure C.12. ${ }^{1} \mathrm{H}$ NMR of compound 4.5 


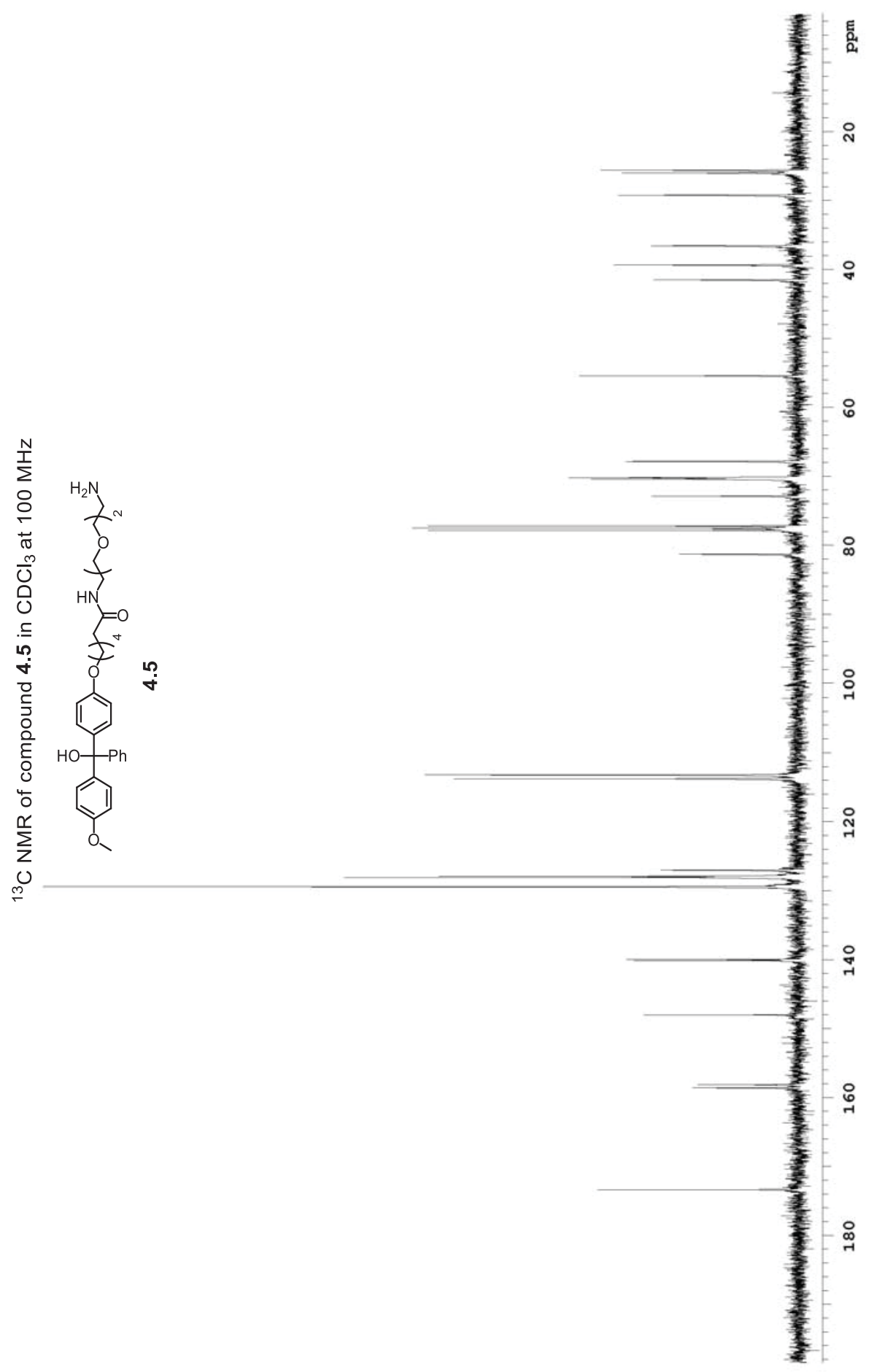

Figure C.13. ${ }^{13} \mathrm{C}$ NMR of compound 4.5 


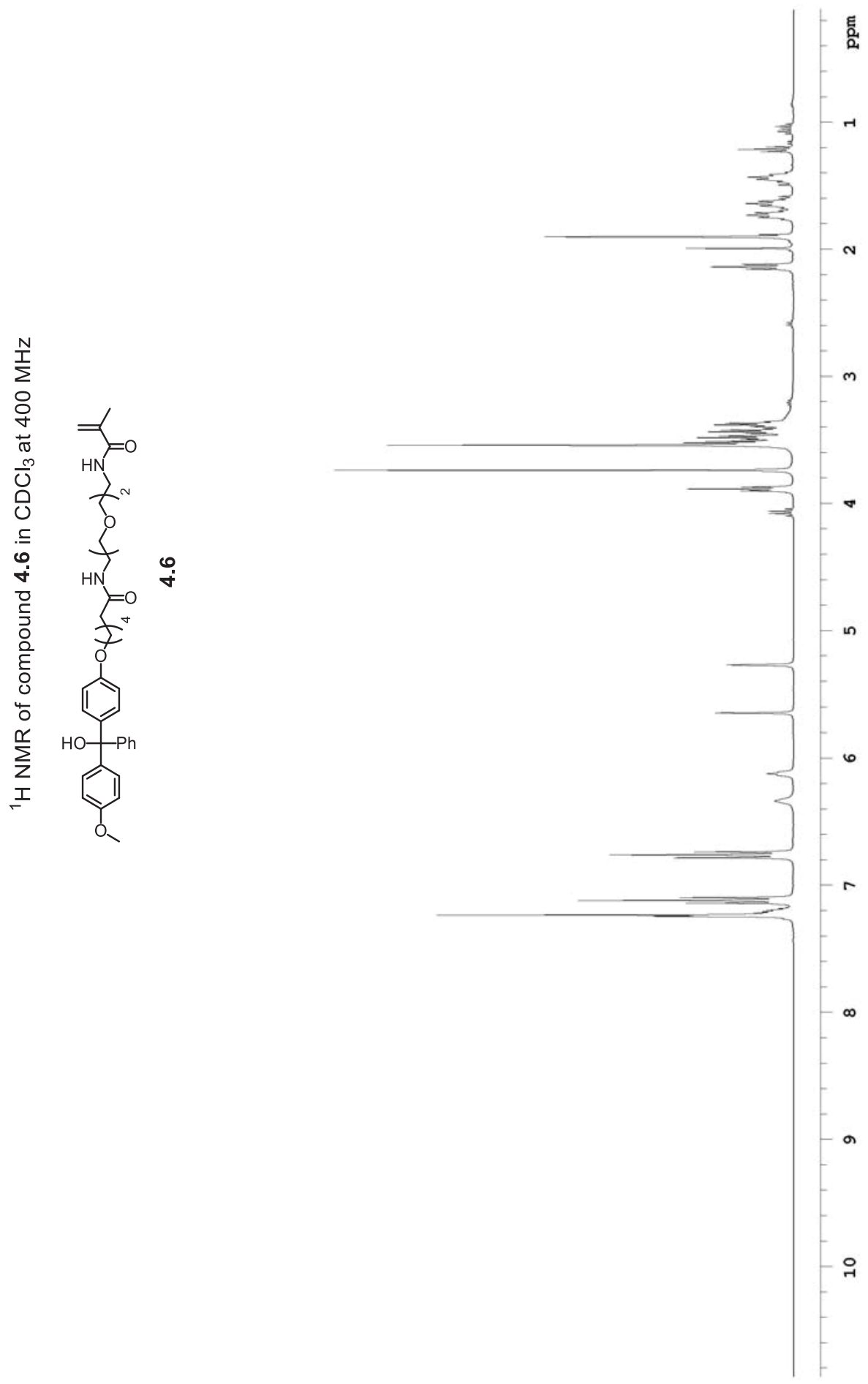

Figure C.14. ${ }^{1} \mathrm{H}$ NMR of compound 4.6 


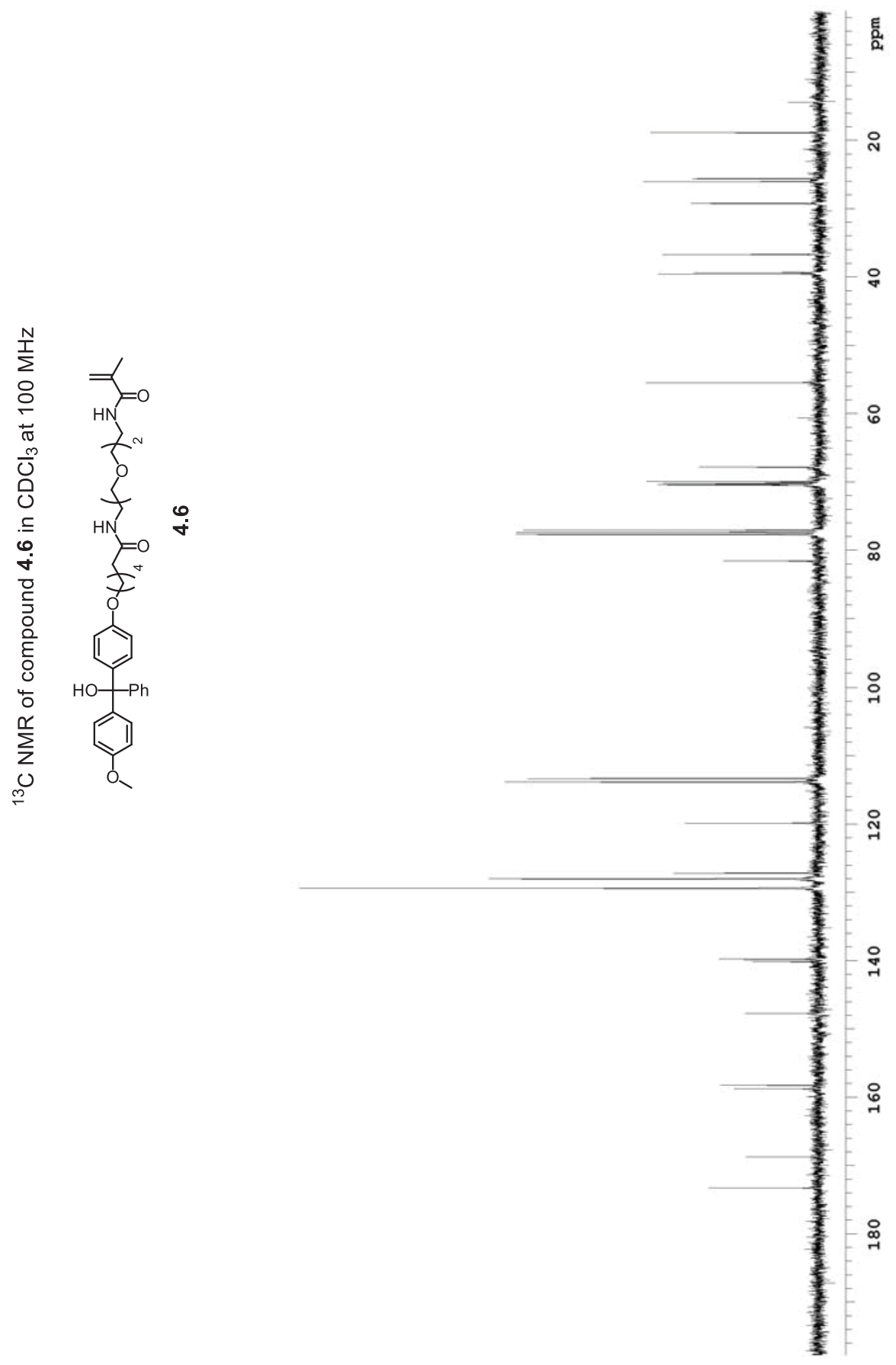

Figure C.15. ${ }^{13} \mathrm{C}$ NMR of compound 4.6 

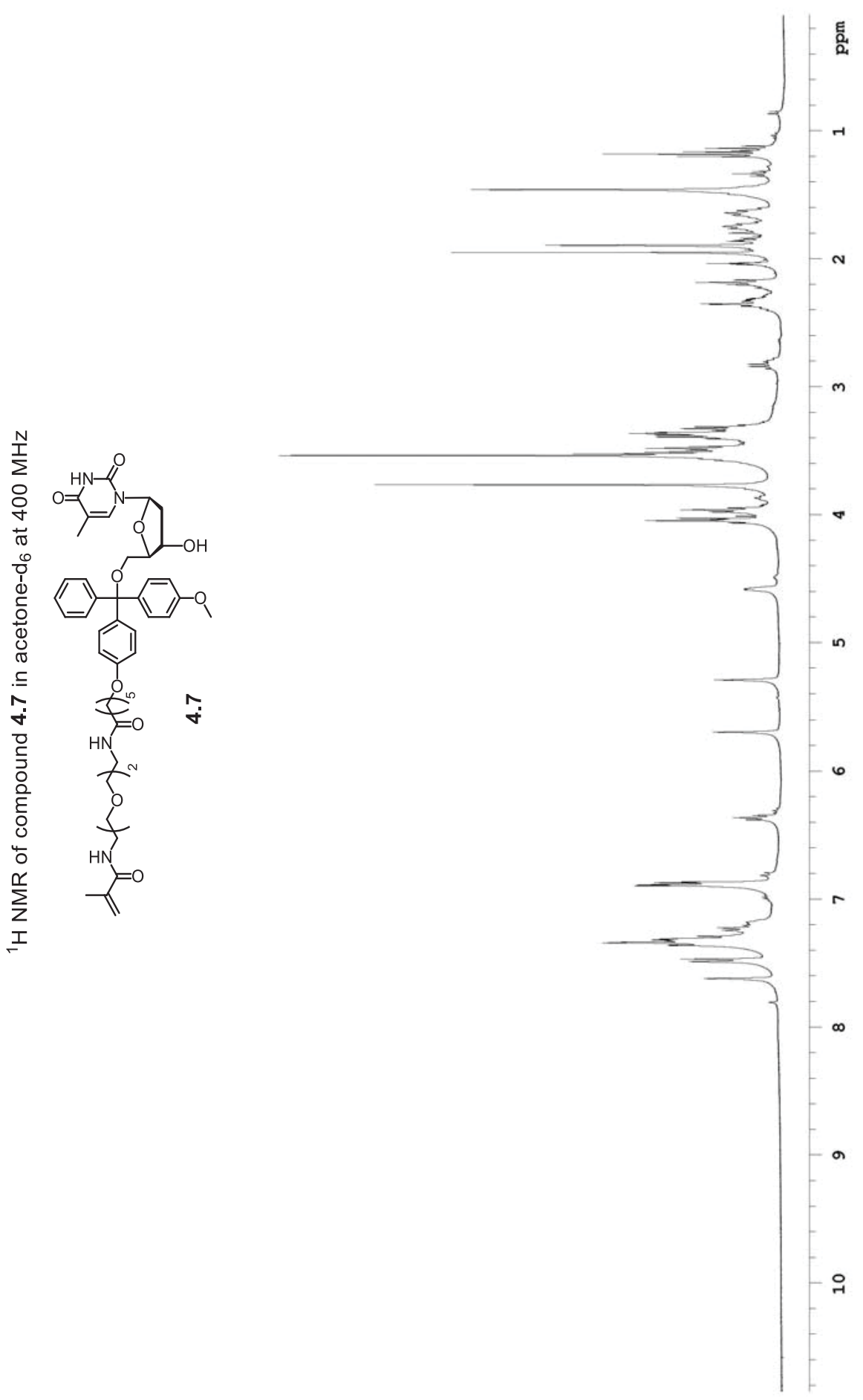

Figure C.16. ${ }^{1} \mathrm{H}$ NMR of compound 4.7 


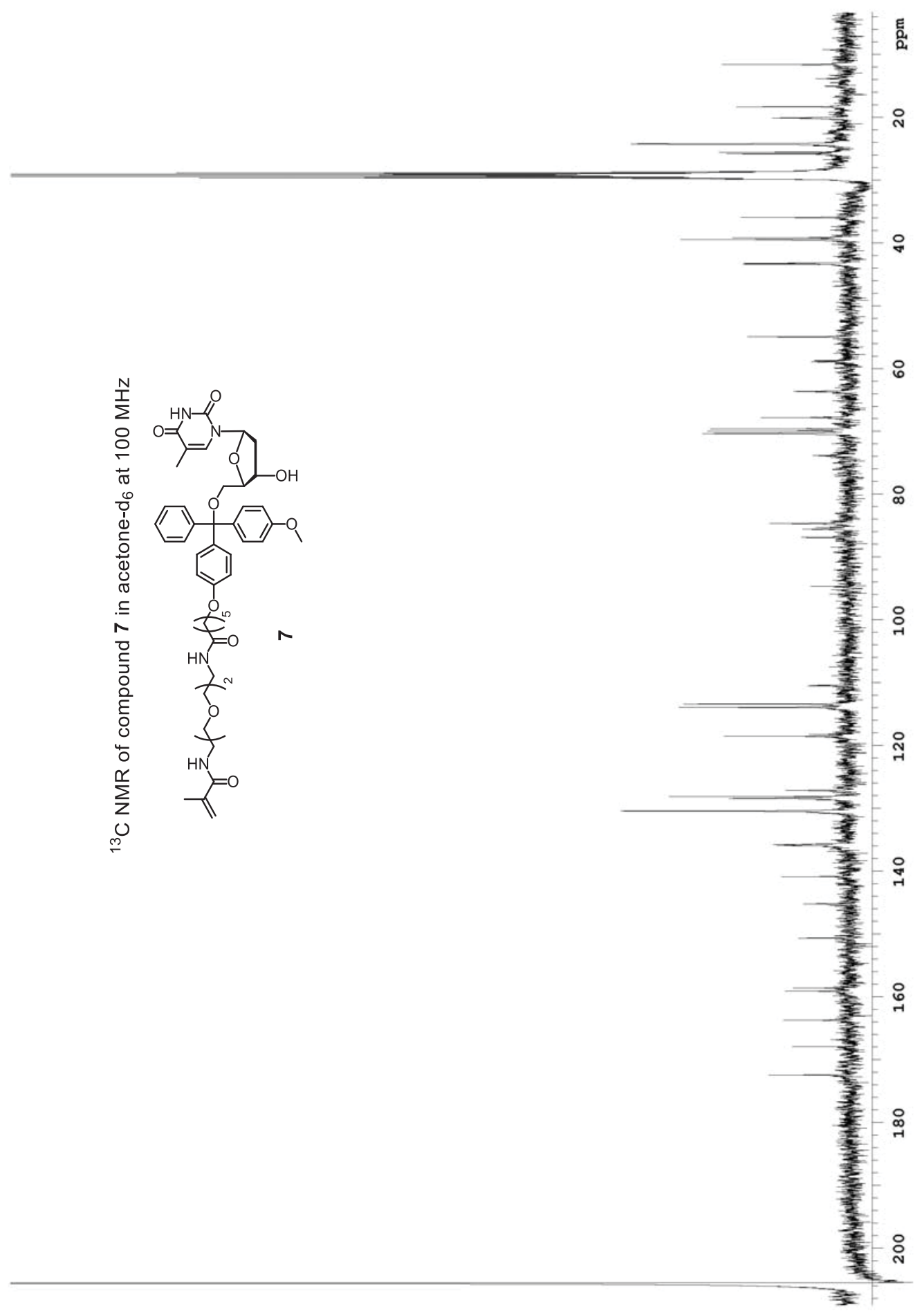

Figure C.17. ${ }^{13} \mathrm{C}$ NMR of compound 4.7 


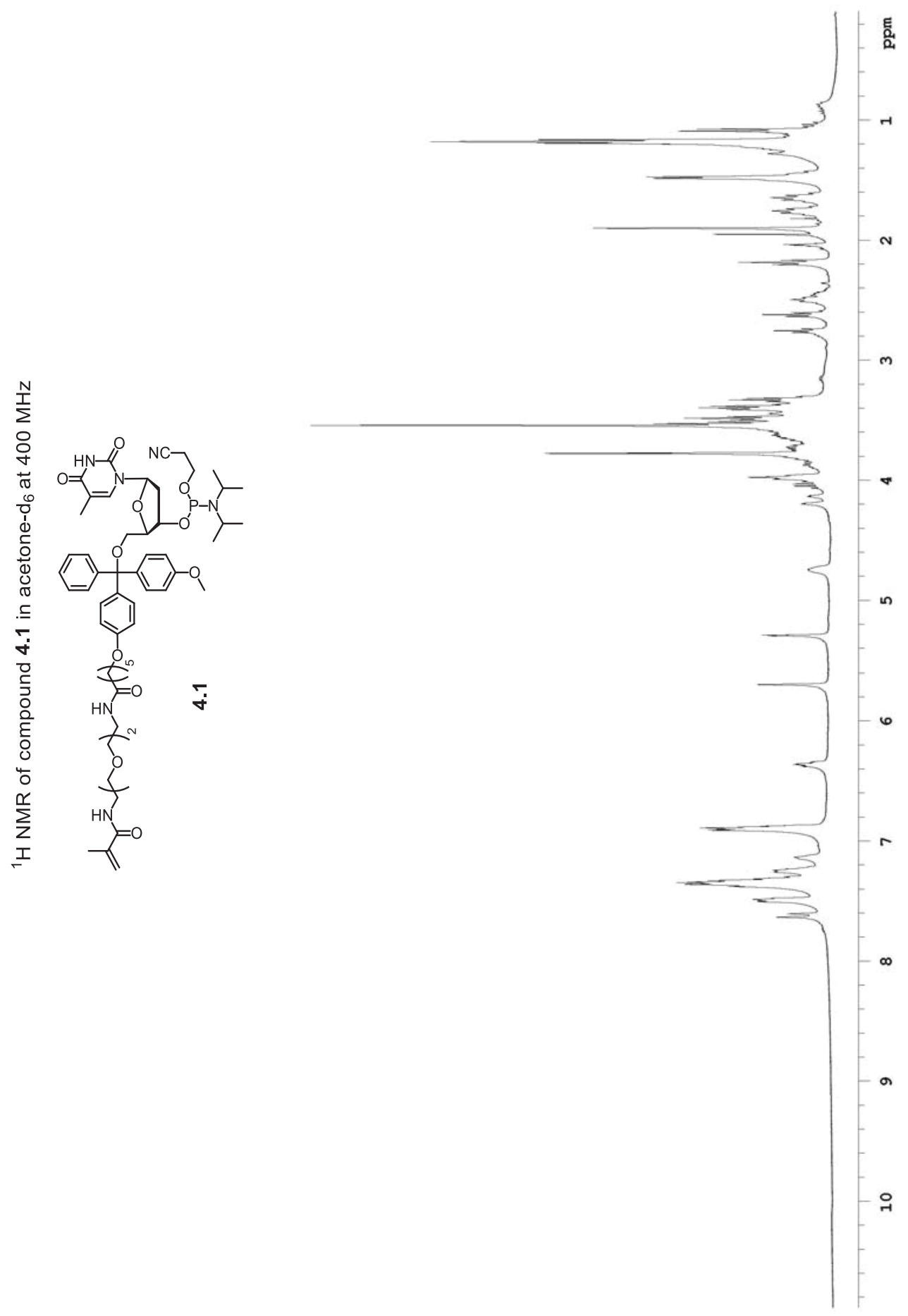

Figure C.18. ${ }^{1} \mathrm{H}$ NMR of compound 4.1 


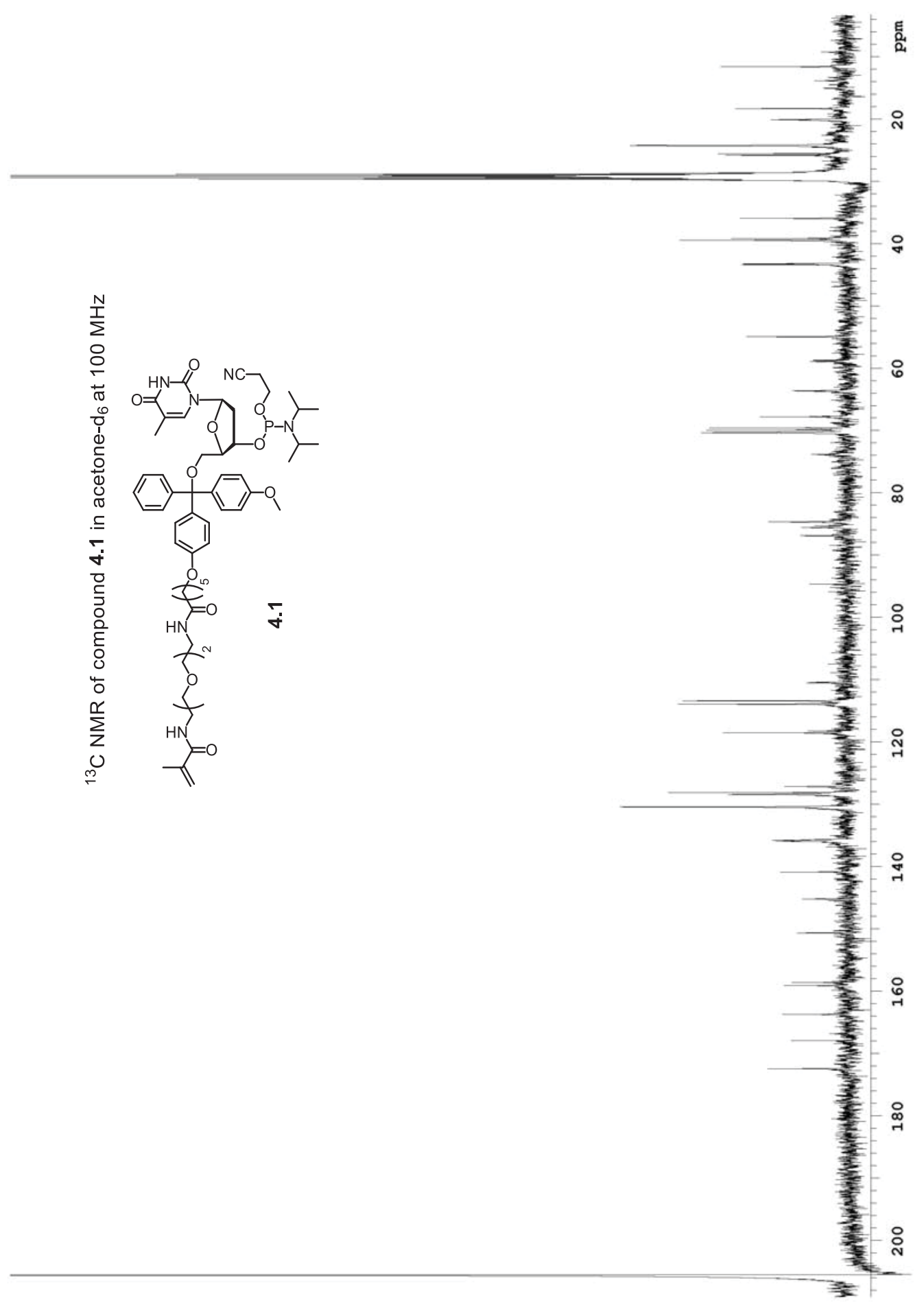

Figure C.19. ${ }^{13} \mathrm{C}$ NMR of compound 4.1 


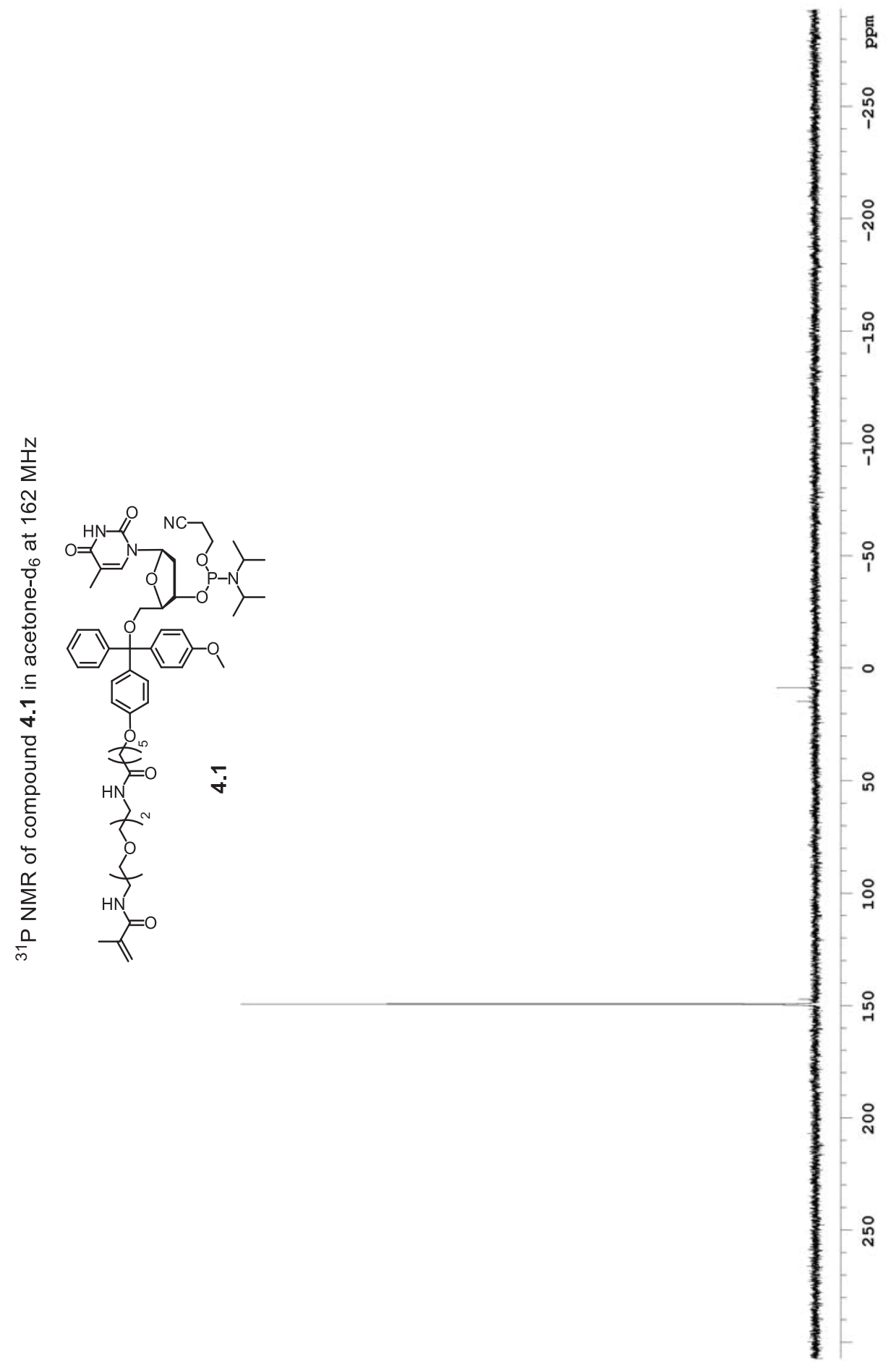

Figure C.20. ${ }^{31} \mathrm{P}$ NMR of compound 4.1 


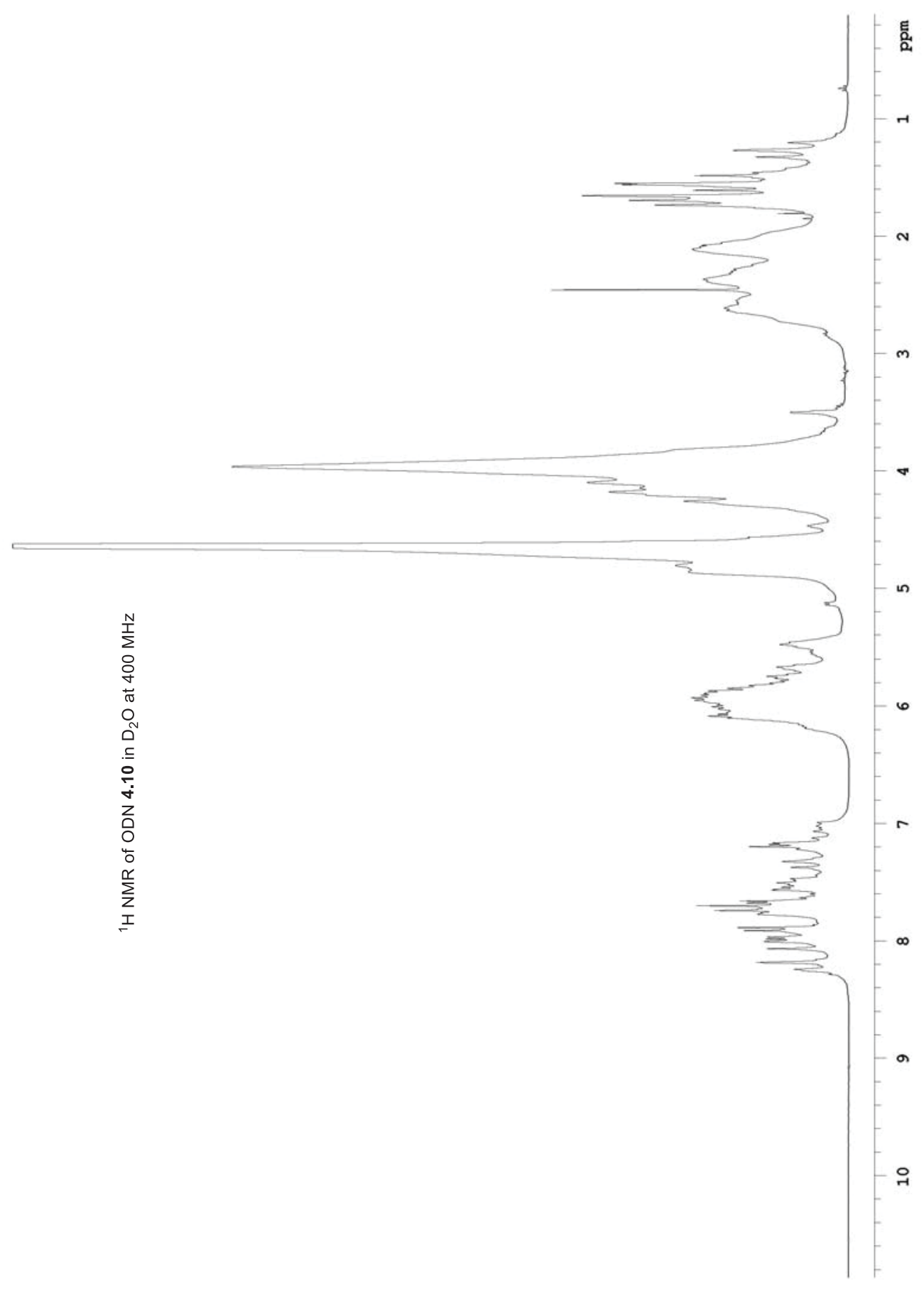

Figure C.21. ${ }^{1} \mathrm{H}$ NMR of ODN 4.10 

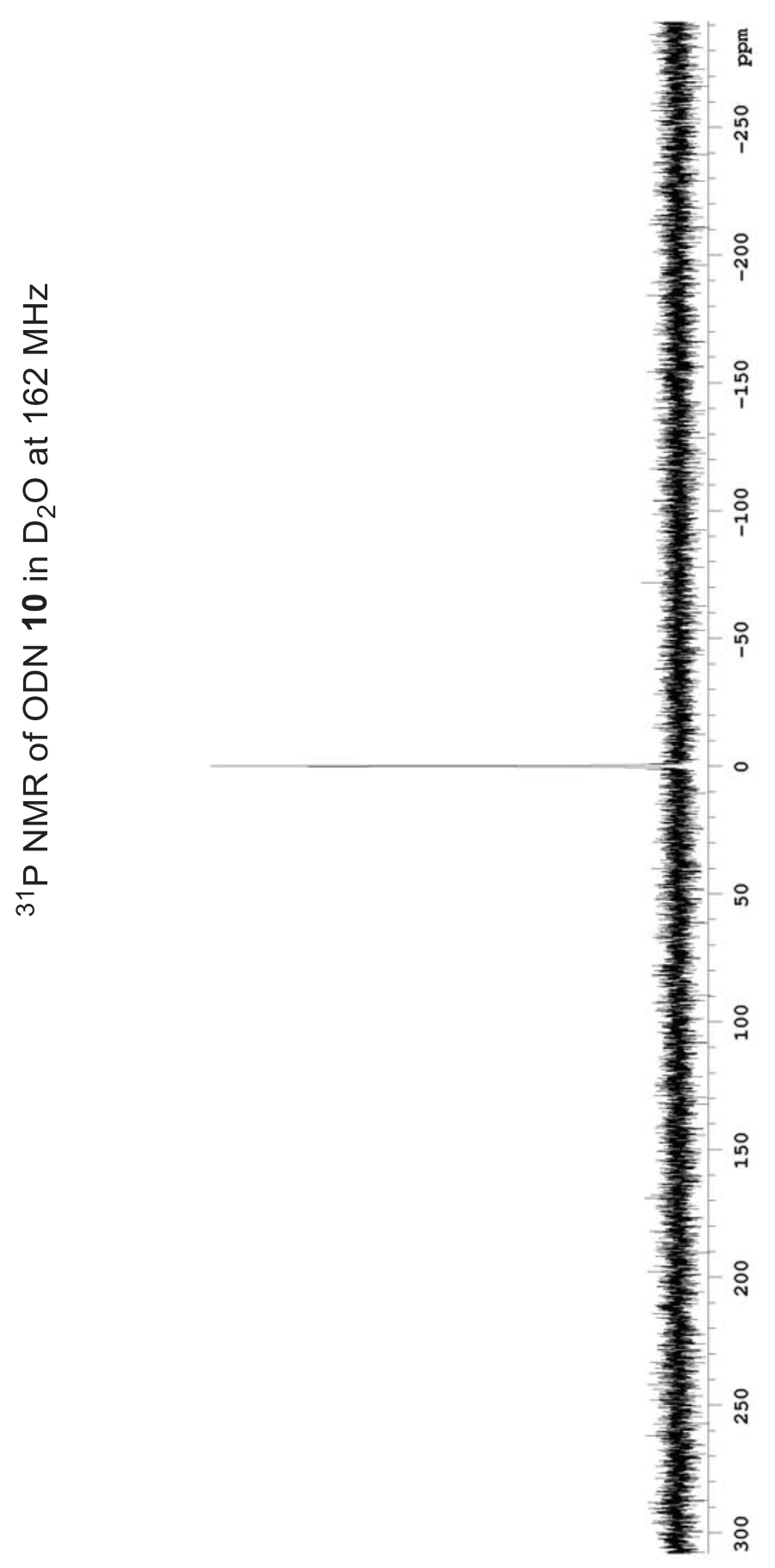

Figure C.22. ${ }^{31} \mathrm{P}$ NMR of ODN 4.10 


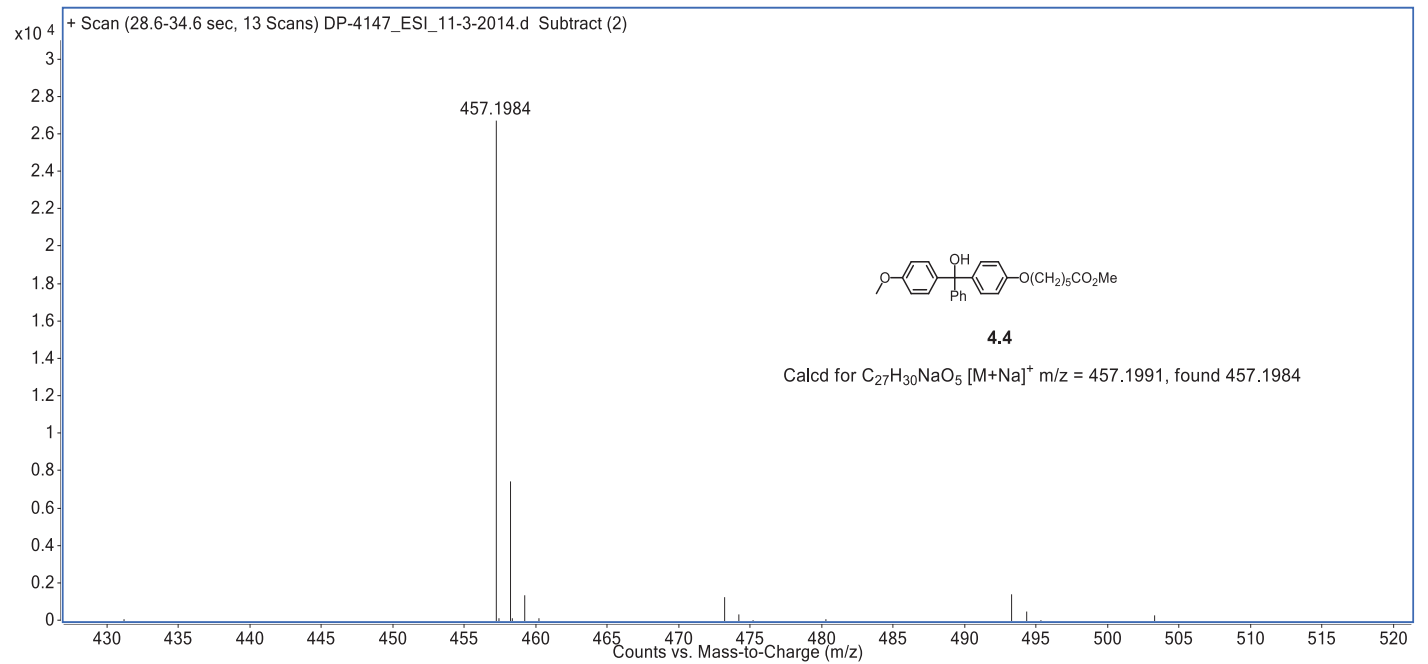

Figure C.23. ESI MS of compound 4.4 


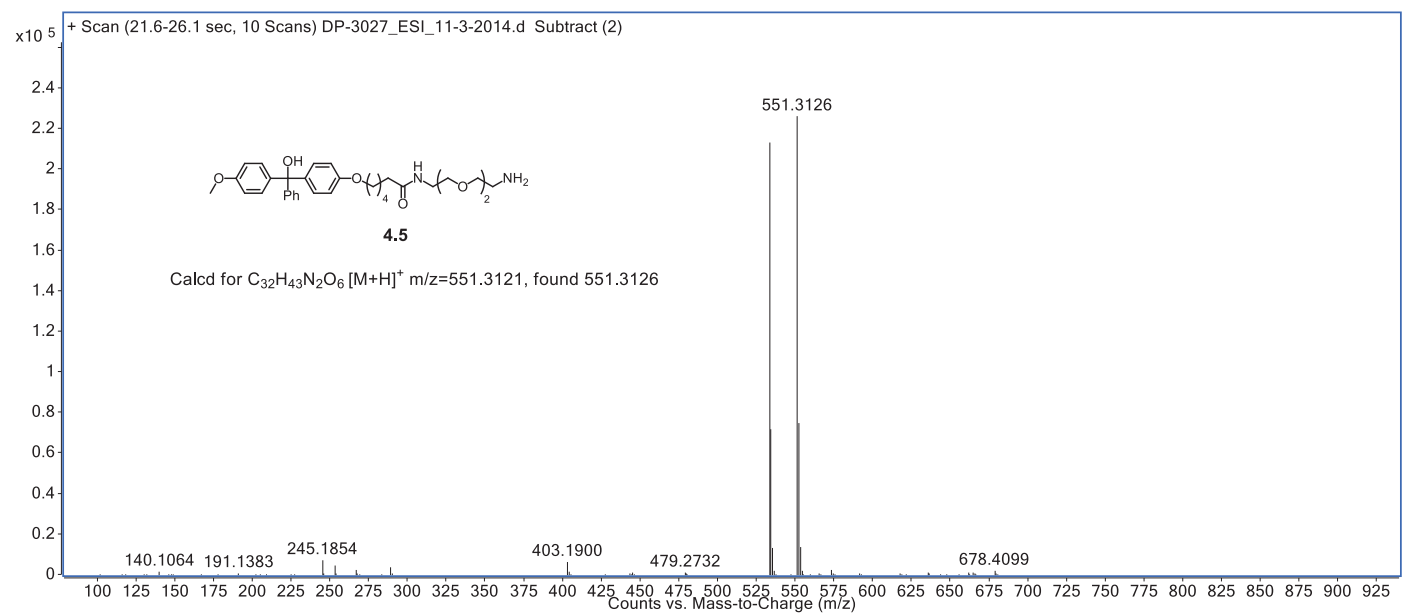

Figure C.24. ESI MS of compound 4.5 


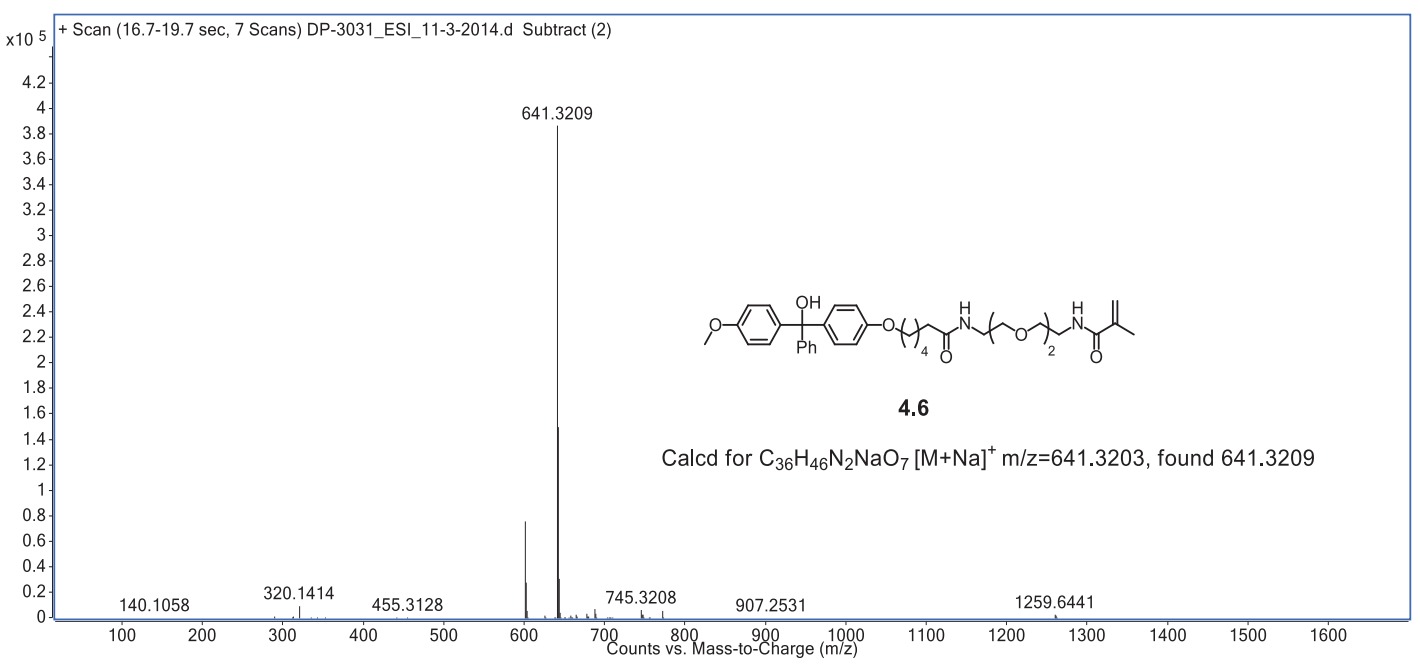

Figure C.25. ESI MS of compound 4.6 


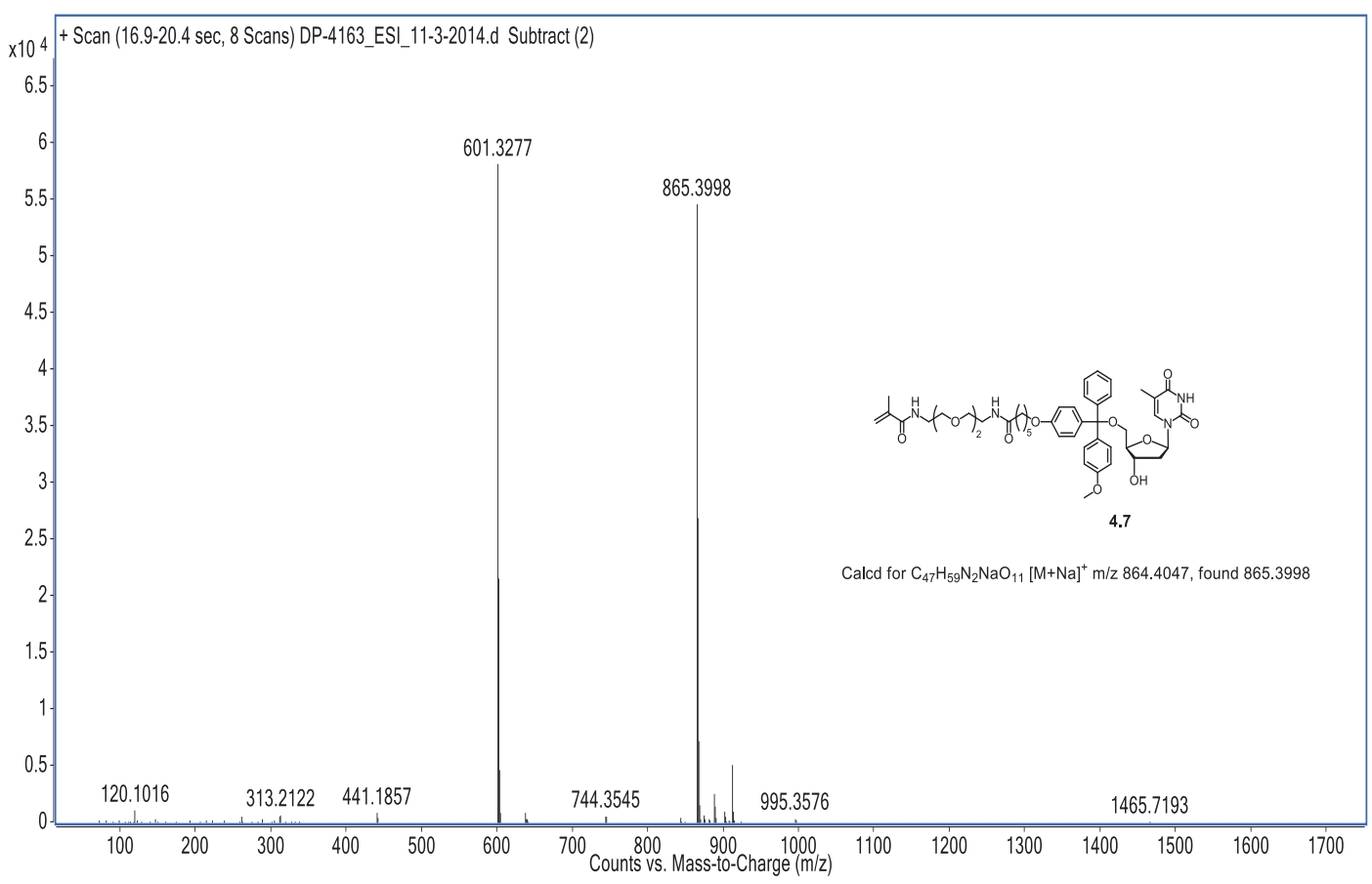

Figure C.26. ESI MS of compound 4.7 


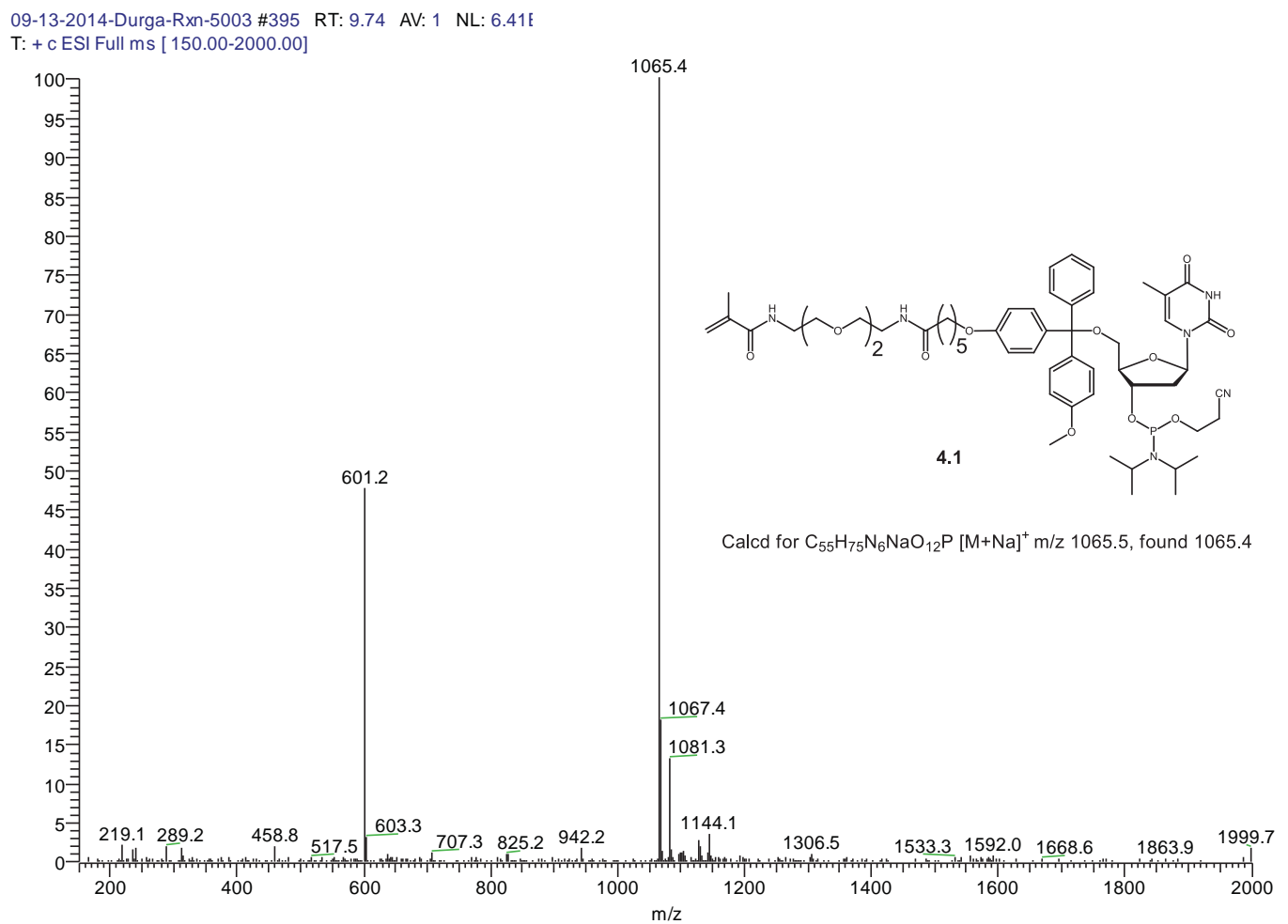

Figure C.27. ESI MS of compound 4.1 


\section{Appendix D}

Supporting Information for Chapter 6

Purification of Synthetic Peptides Using a Catching Full-Length

Sequence by Polymerization Approach 


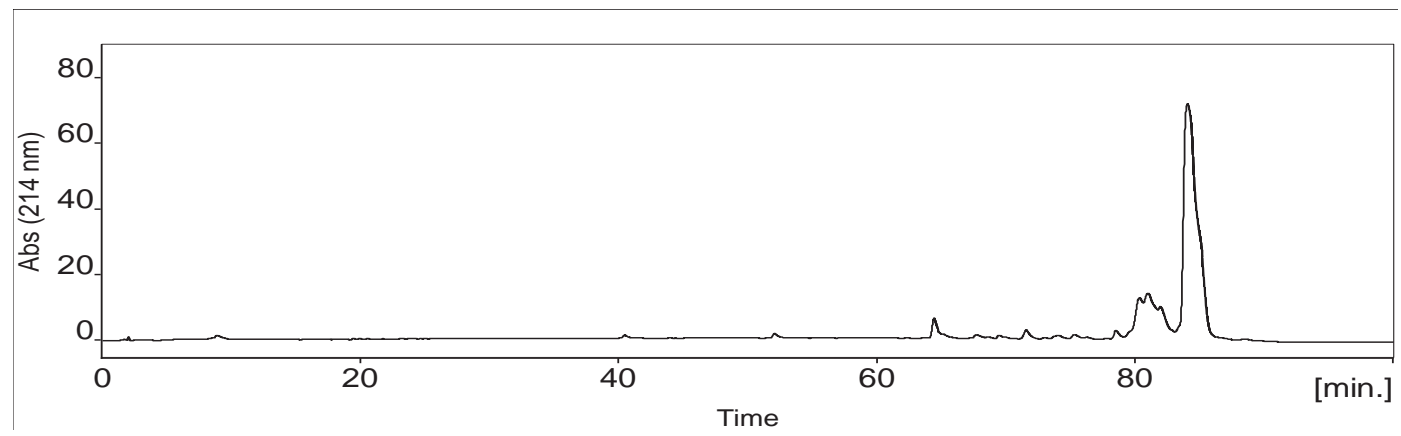

Figure D.1. RP HPLC profile of crude peptide 6.11 (16-amino acid) with side chain protecting groups and methacrylamide tag on

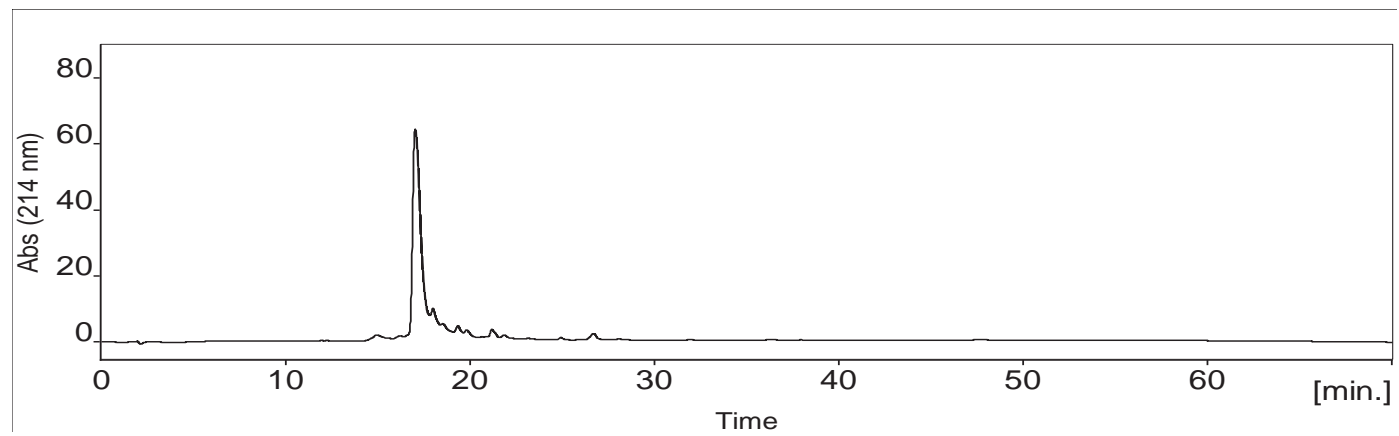

Figure D.2. RP HPLC profile of crude peptide 6.11 (16-amino acid) with side chain protecting groups and methacrylamide tag off 


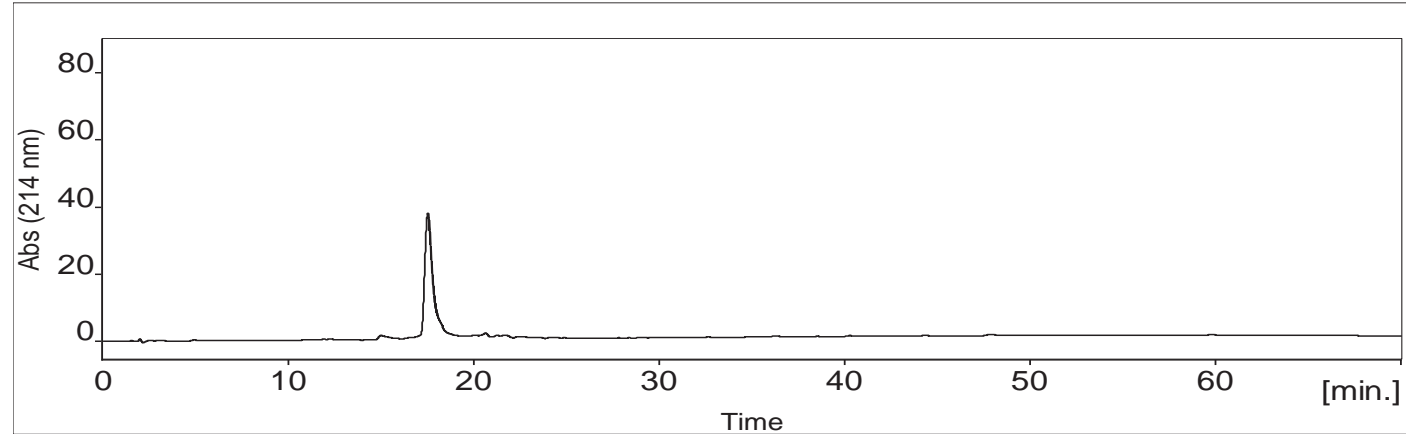

Figure D.3. RP HPLC profile of peptide 6.11 (16-amino acid) purified by catching full-length sequences by polymerization 


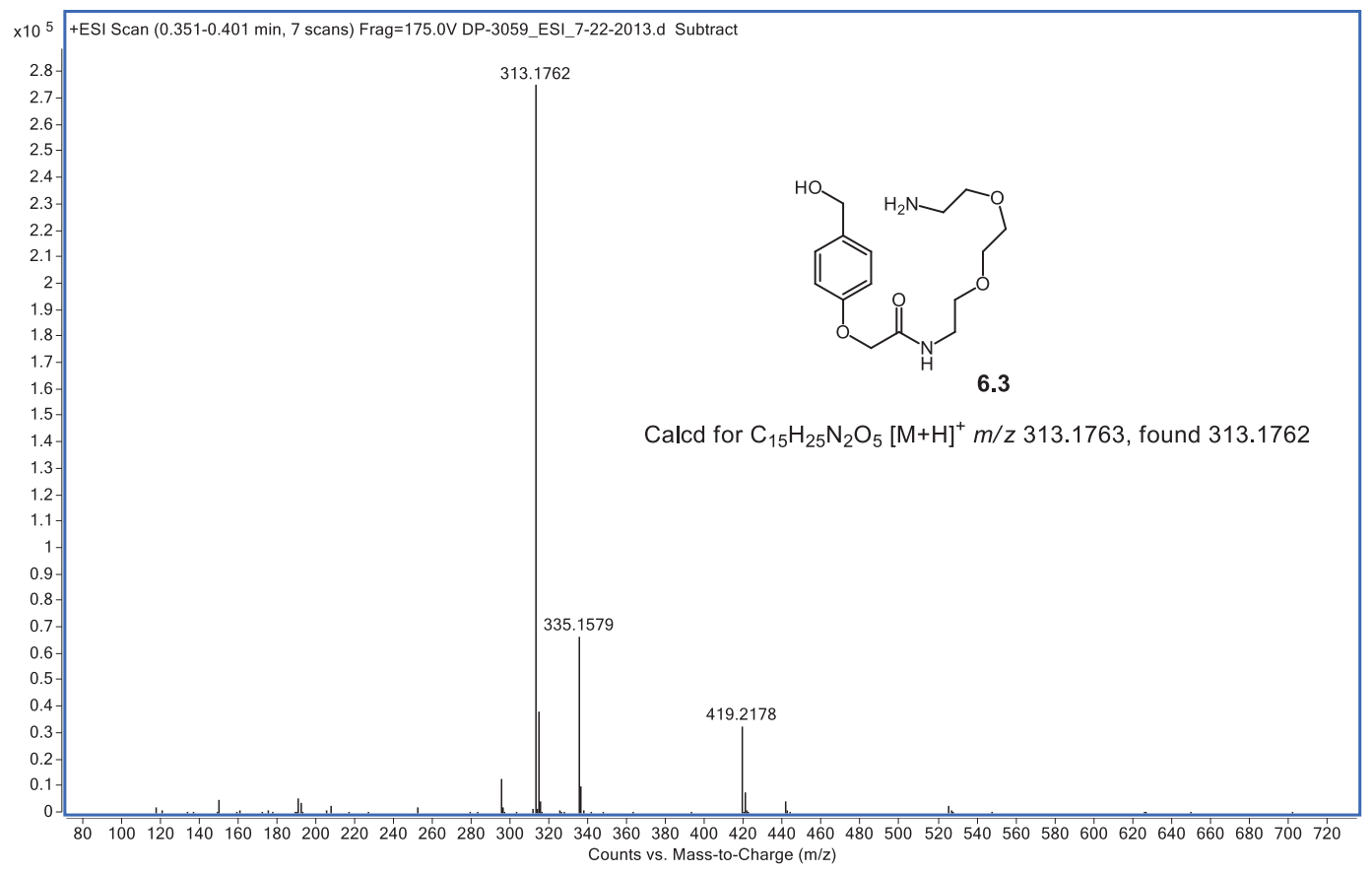

Figure D.4. ESI MS of compound 6.3 


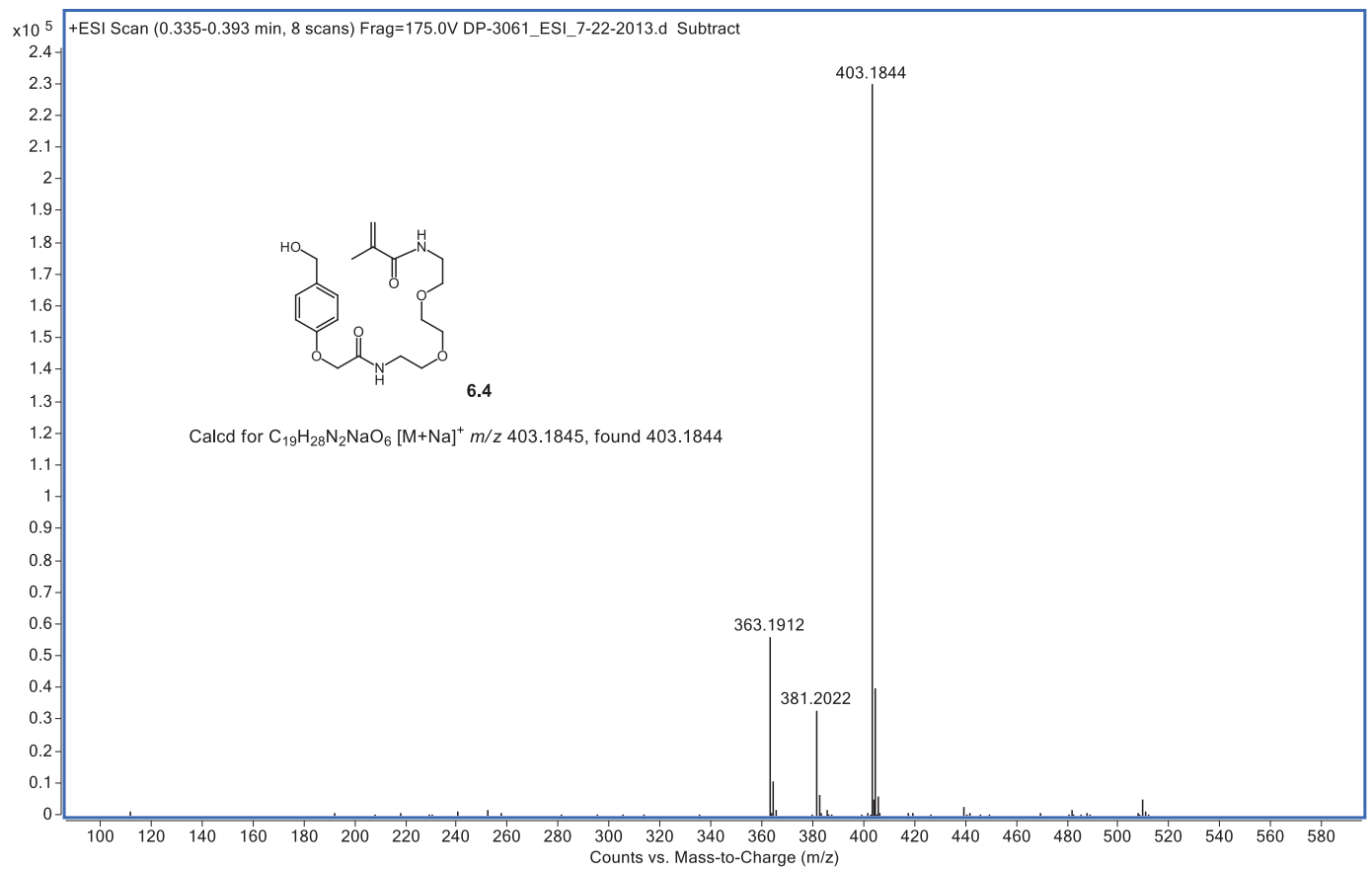

Figure D.5. ESI MS of compound 6.4 

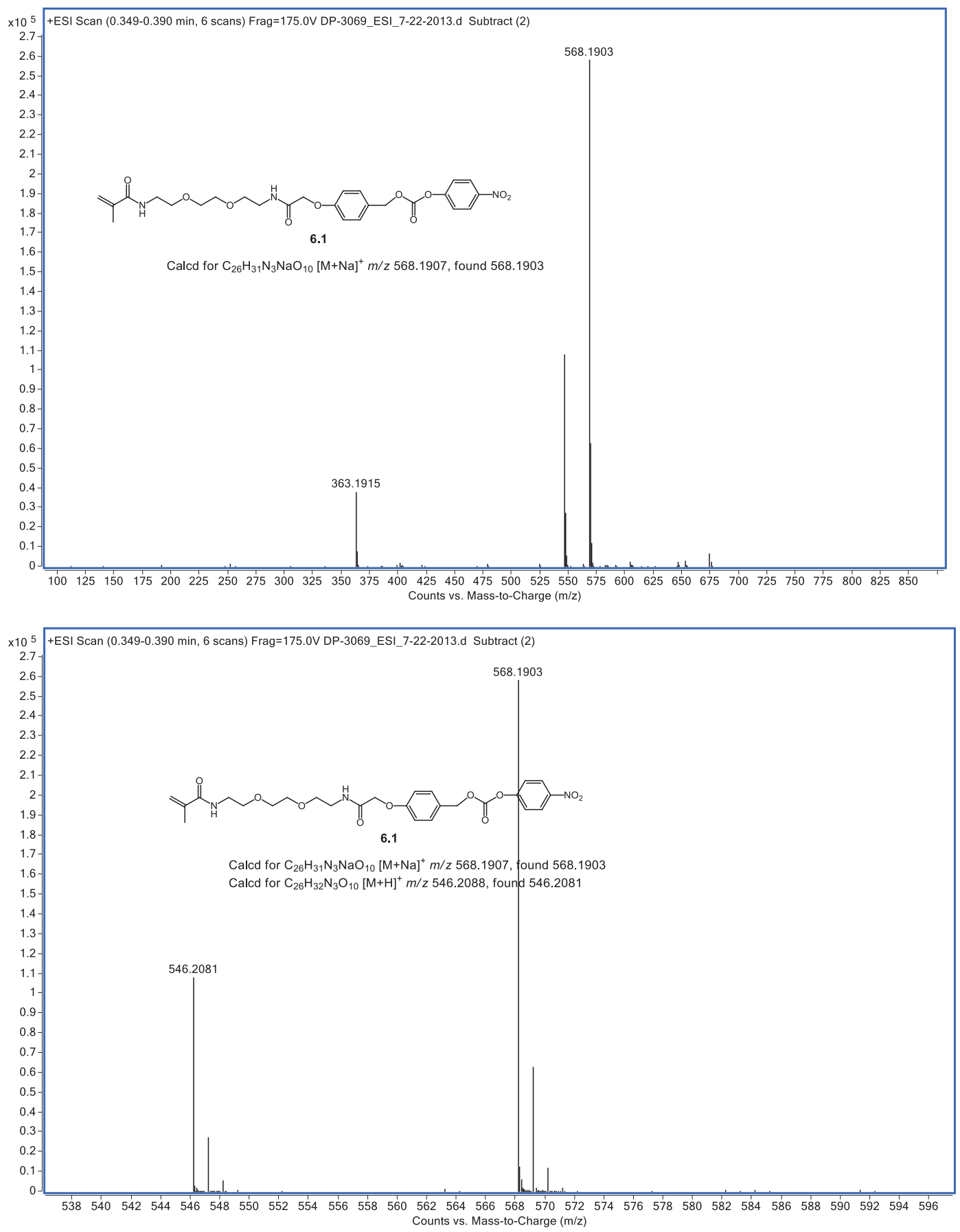

Figure D.6. ESI MS of compound 6.1 


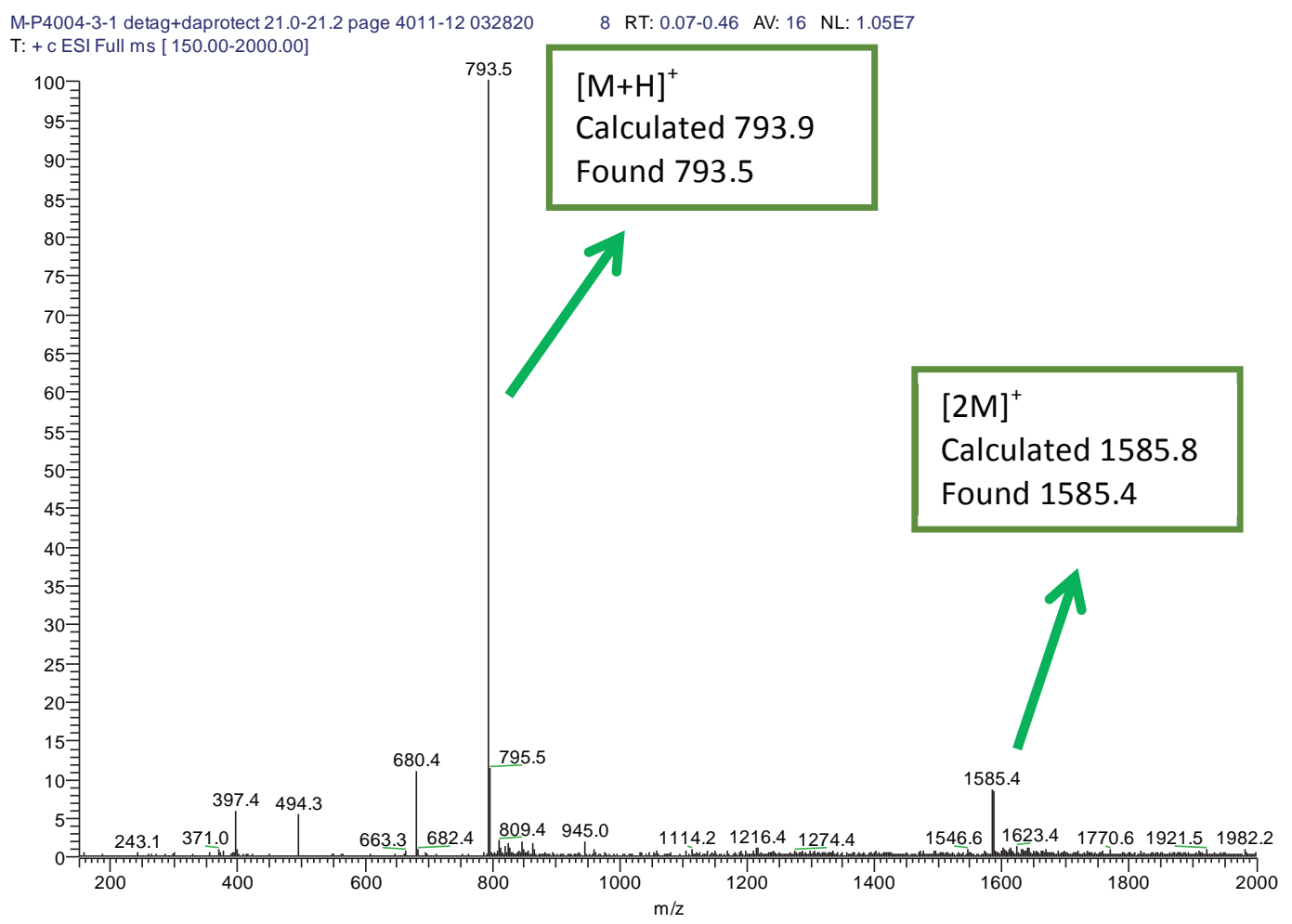

M-P4004-3-1 detag+daprotect 21.0-21.2 page 4011-12 032820 8 RT: 0.07-0.46 AV: 16 NL: 1.05E7

T: + c ESI Full ms [150.00-2000.00]

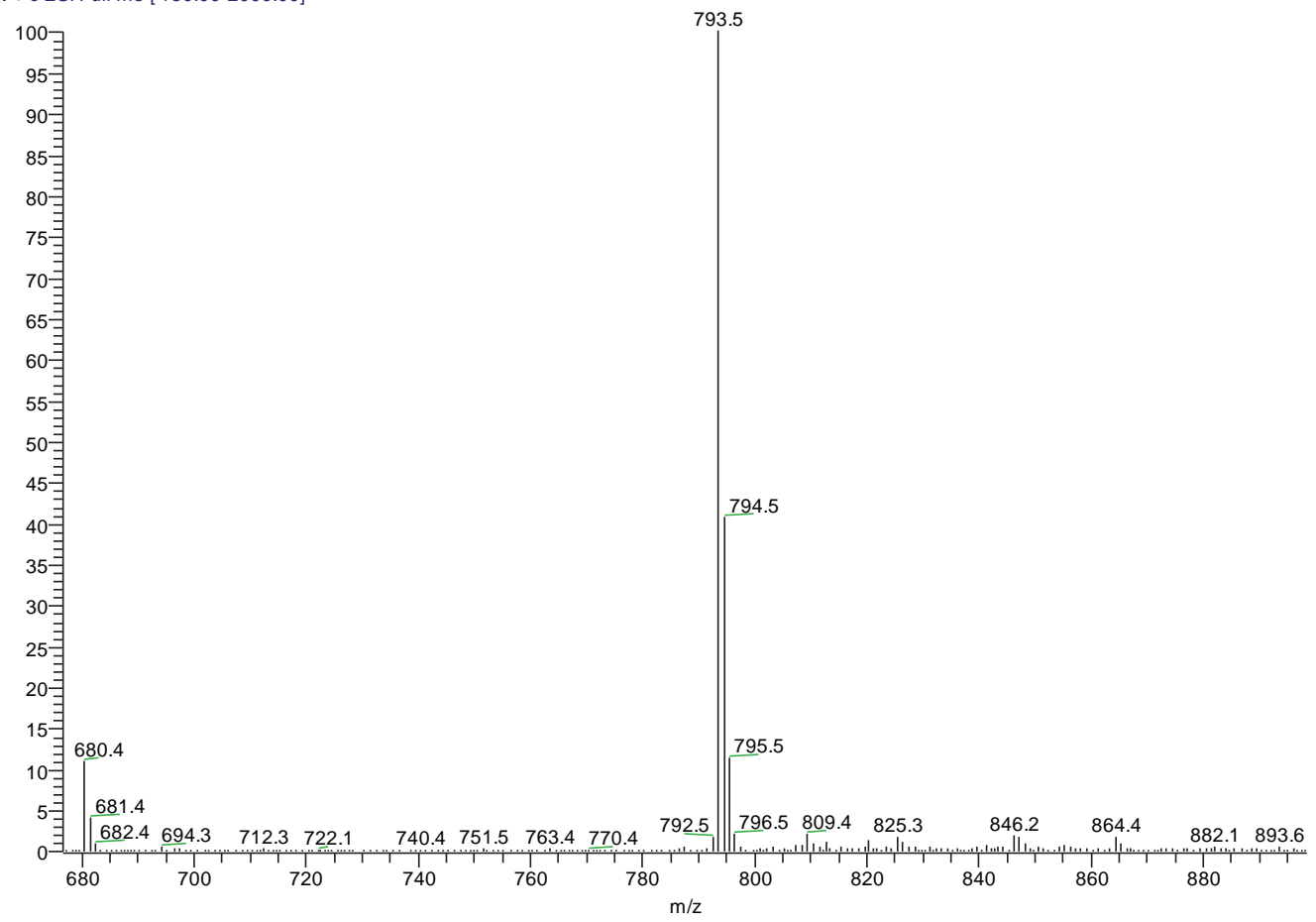

Figure D.7. ESI MS of the 6-amino acid peptide 6.5 


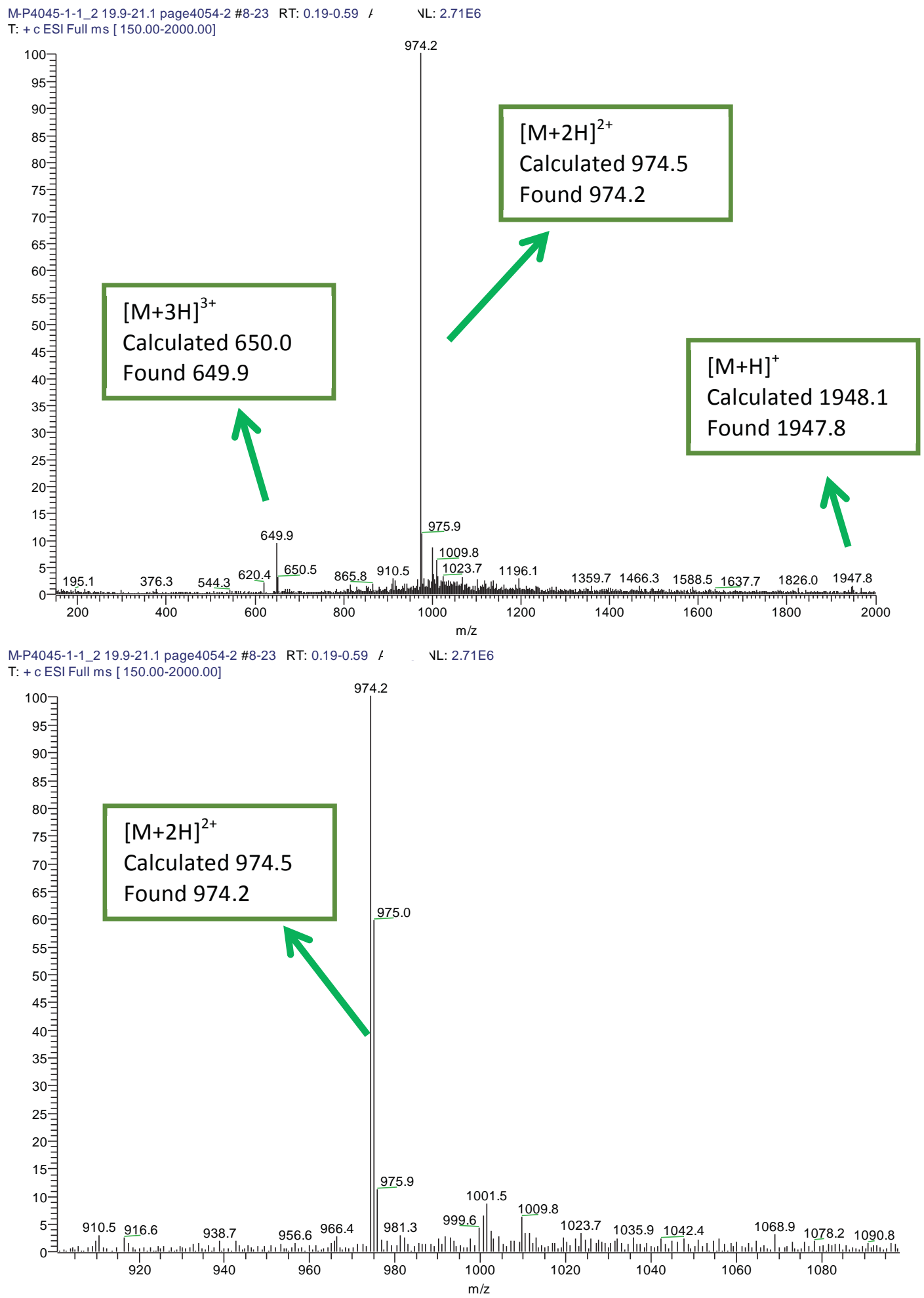

Figure D.8. ESI MS of the 16-amino acid peptide $\mathbf{6 . 1 1}$ 


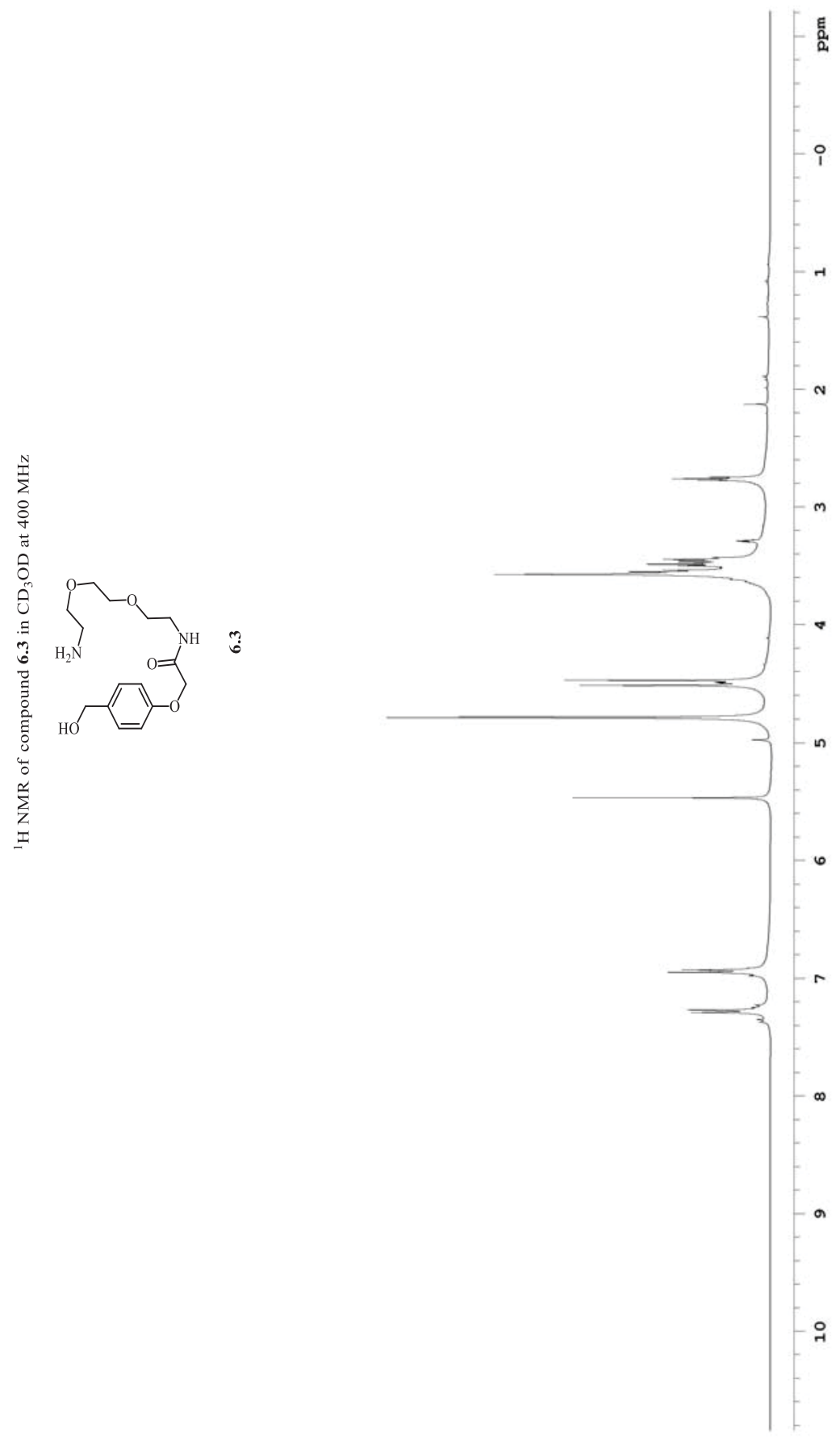

Figure D.9. ${ }^{1} \mathrm{H}$ NMR of compound 6.3 


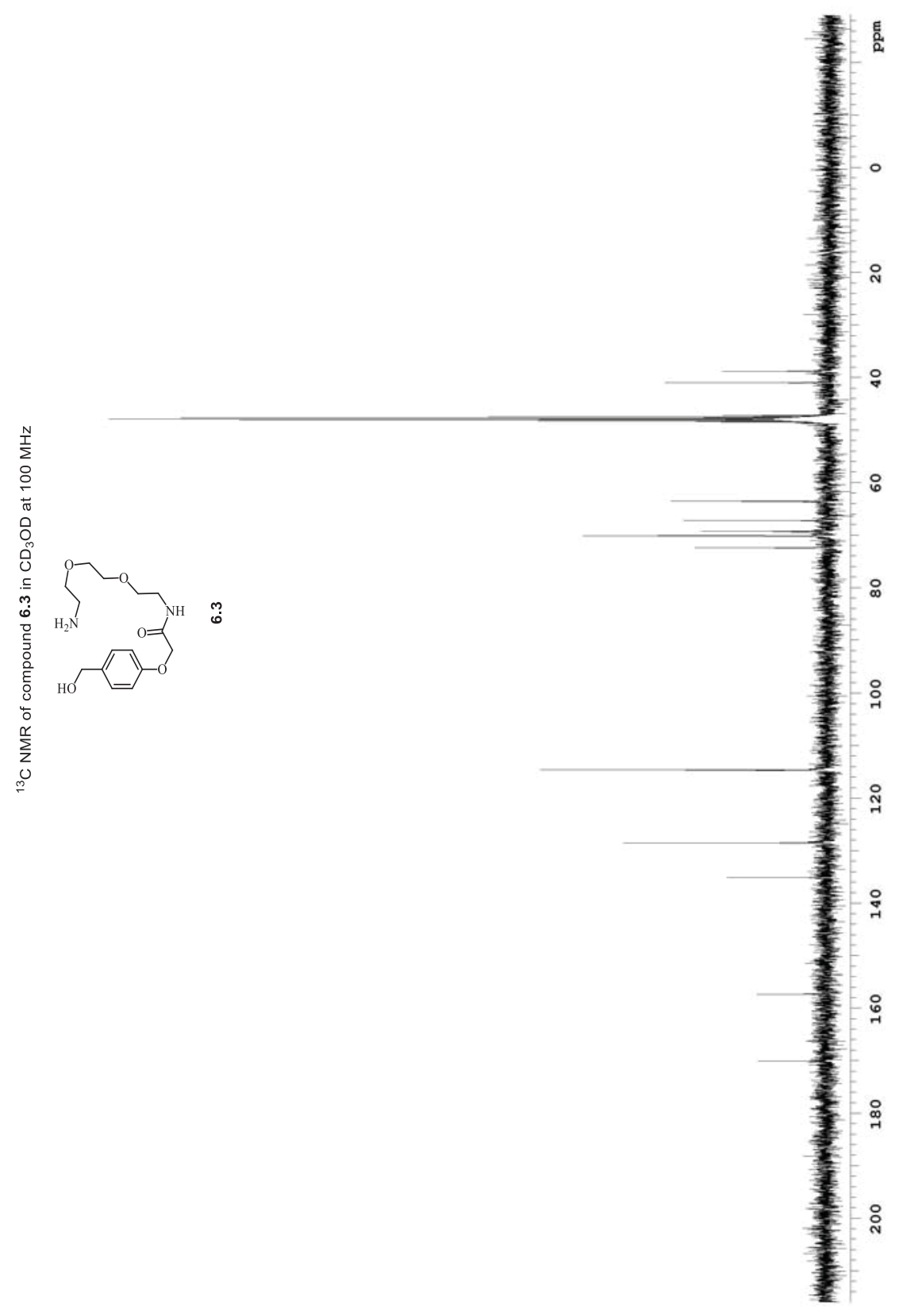

Figure D.10. ${ }^{13} \mathrm{C}$ NMR of compound 6.3 


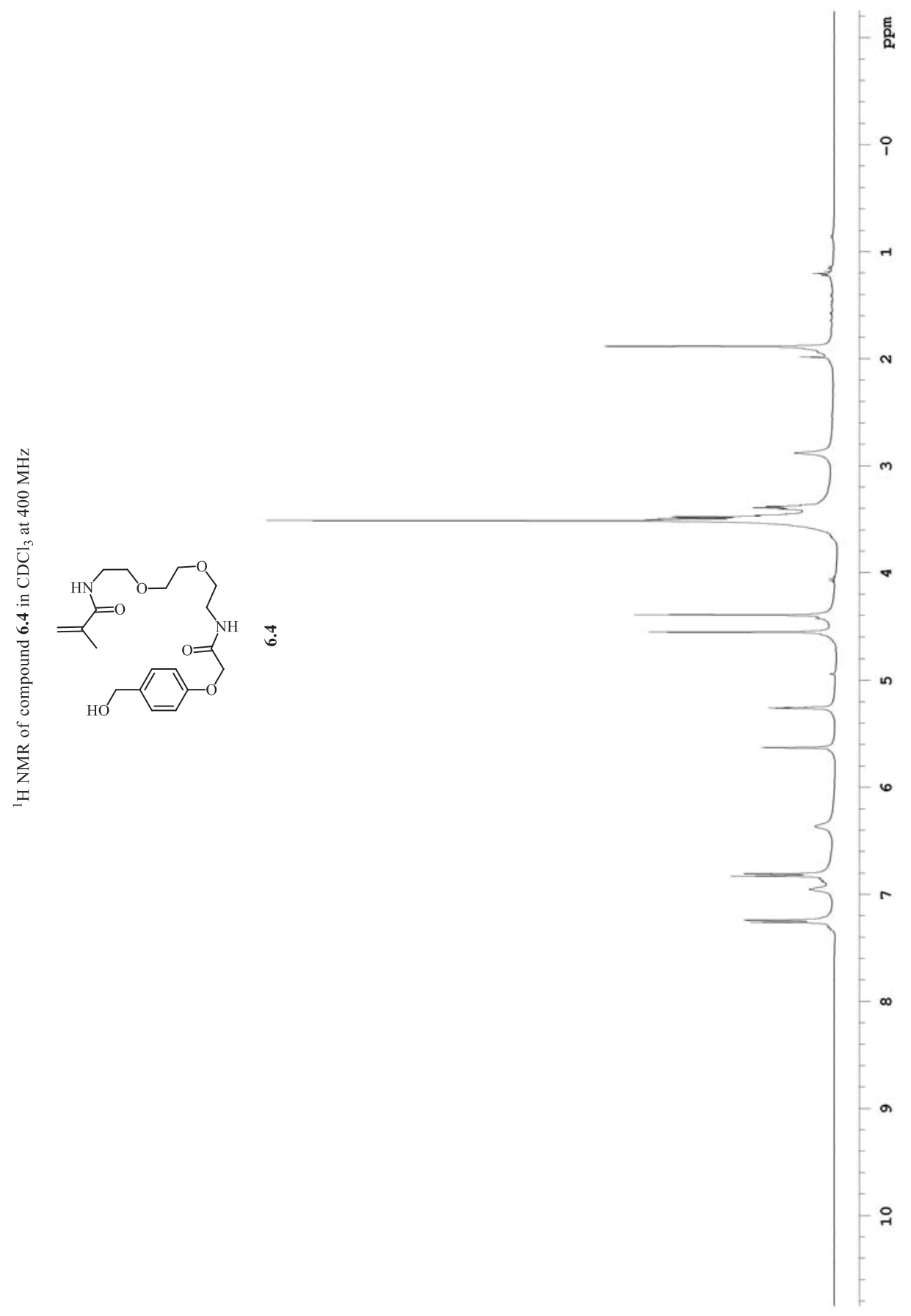

Figure D.11. ${ }^{1} \mathrm{H}$ NMR of compound 6.4 


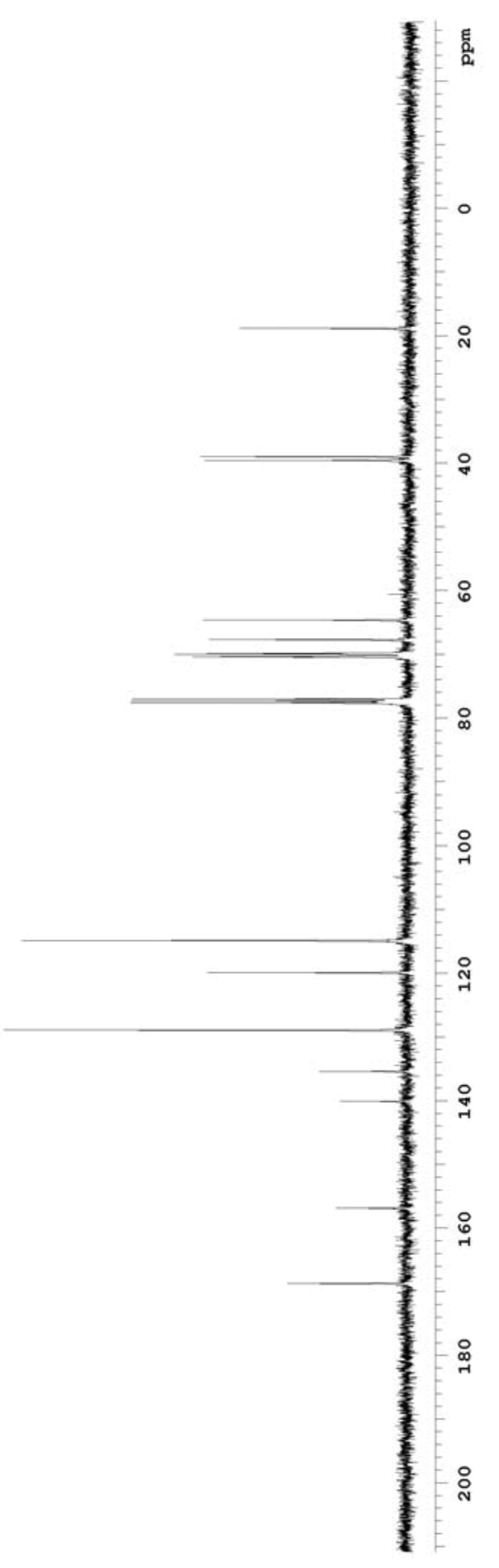

Figure D.12. ${ }^{13} \mathrm{C}$ NMR of compound 6.4 


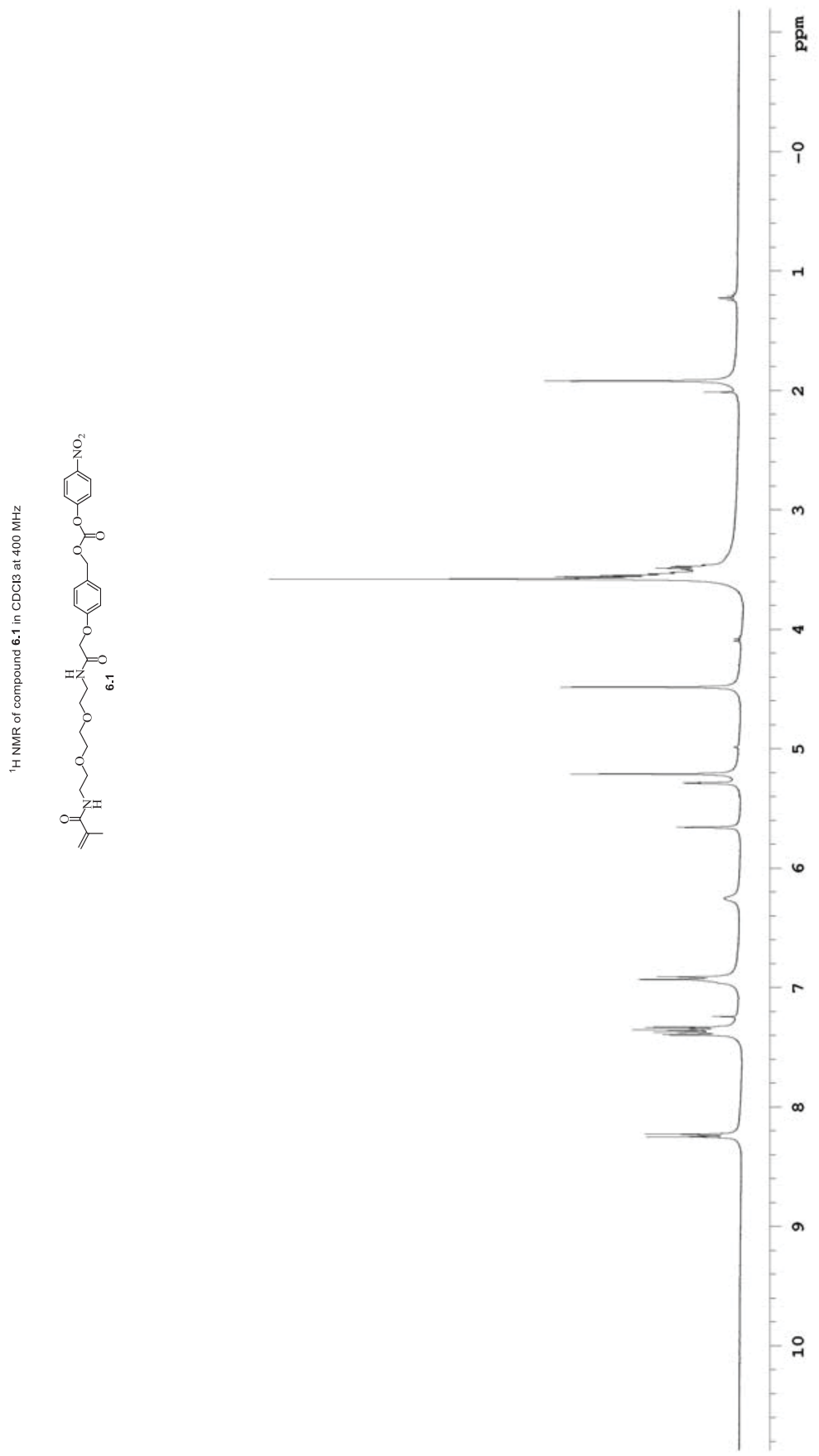

Figure D.13. ${ }^{1} \mathrm{H}$ NMR of compound 6.1 


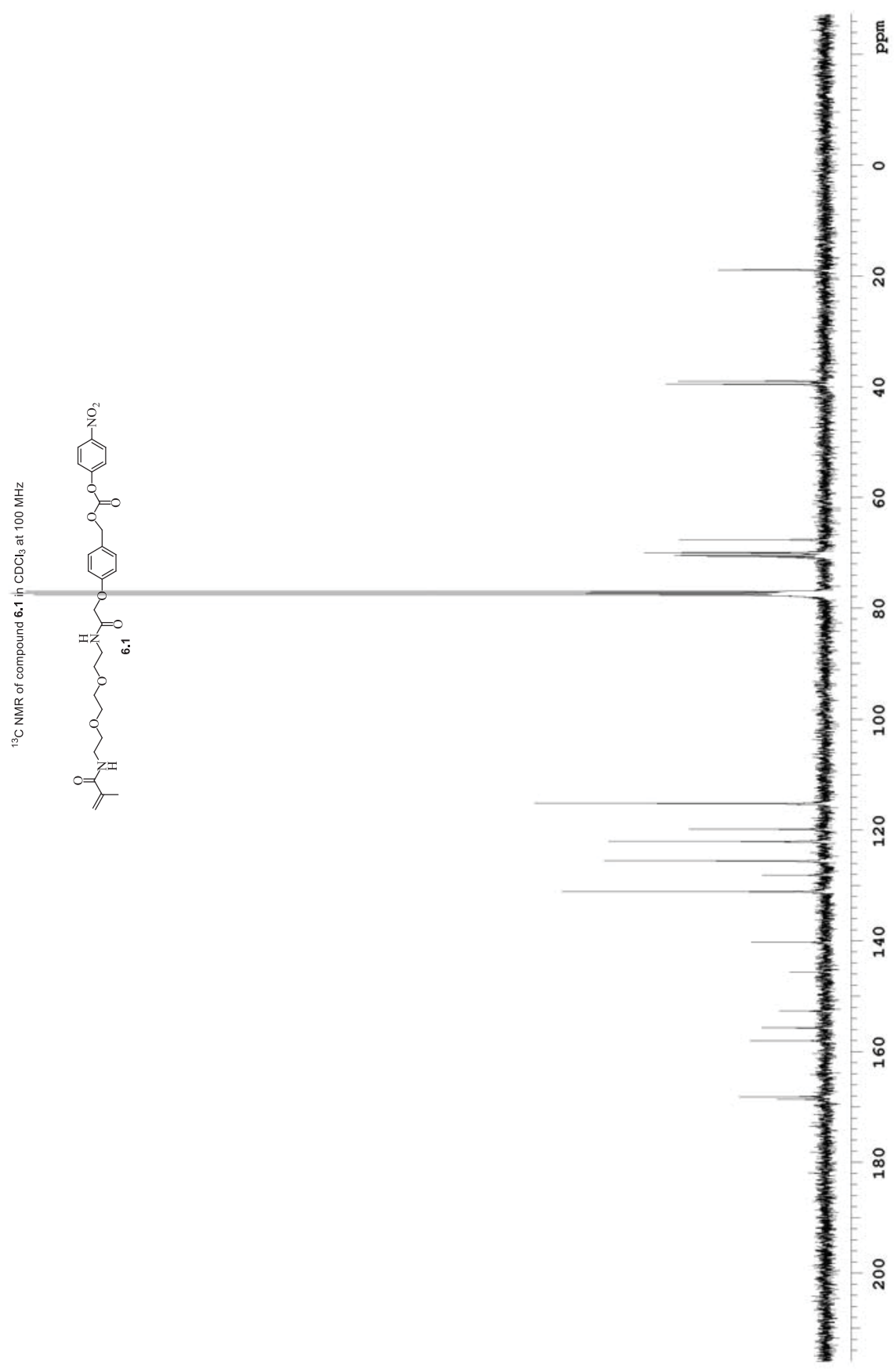

Figure D.14. ${ }^{13} \mathrm{C}$ NMR of compound 6.1 


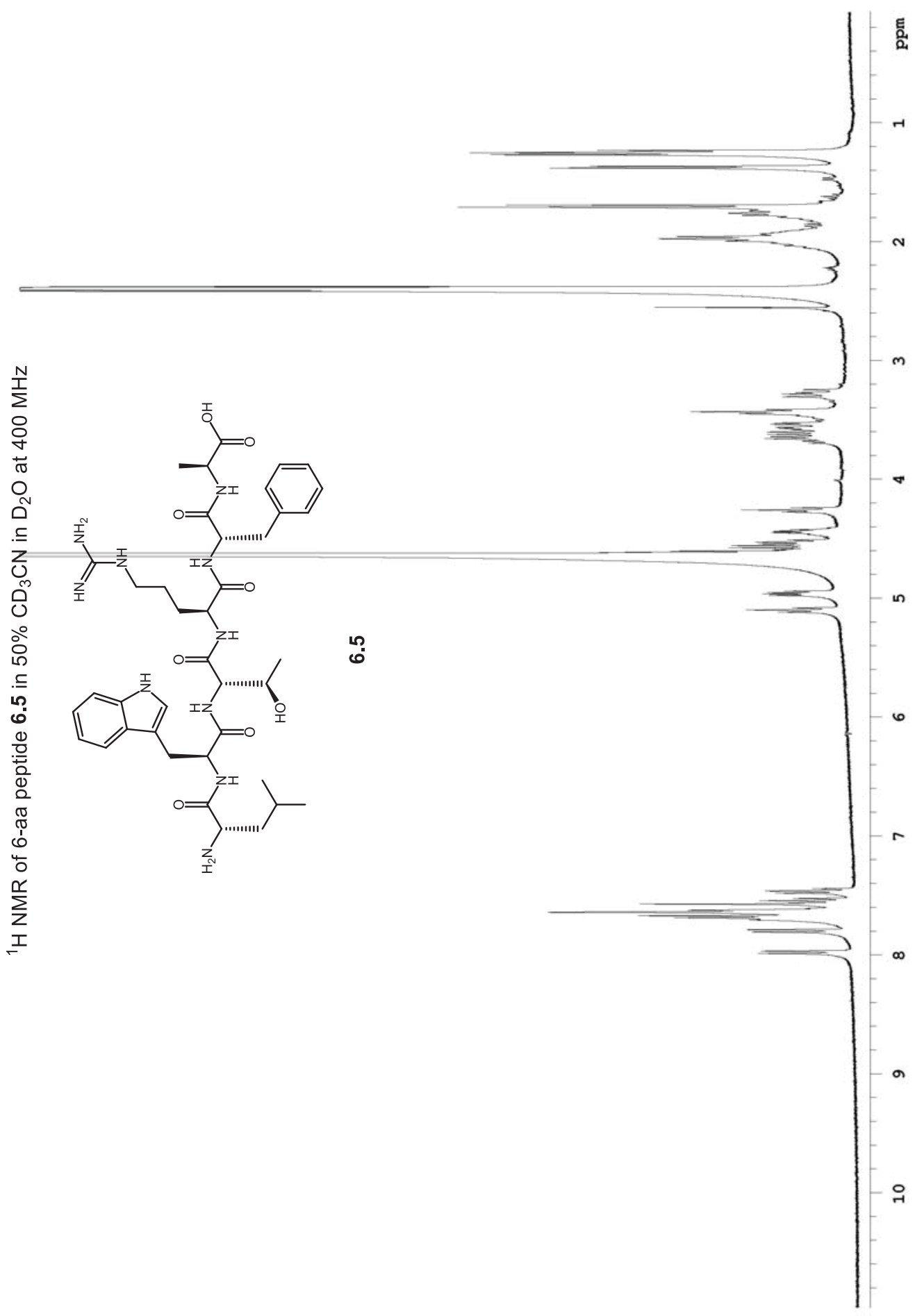

Figure D.15. ${ }^{1}$ H NMR of 6-aa peptide 6.5 


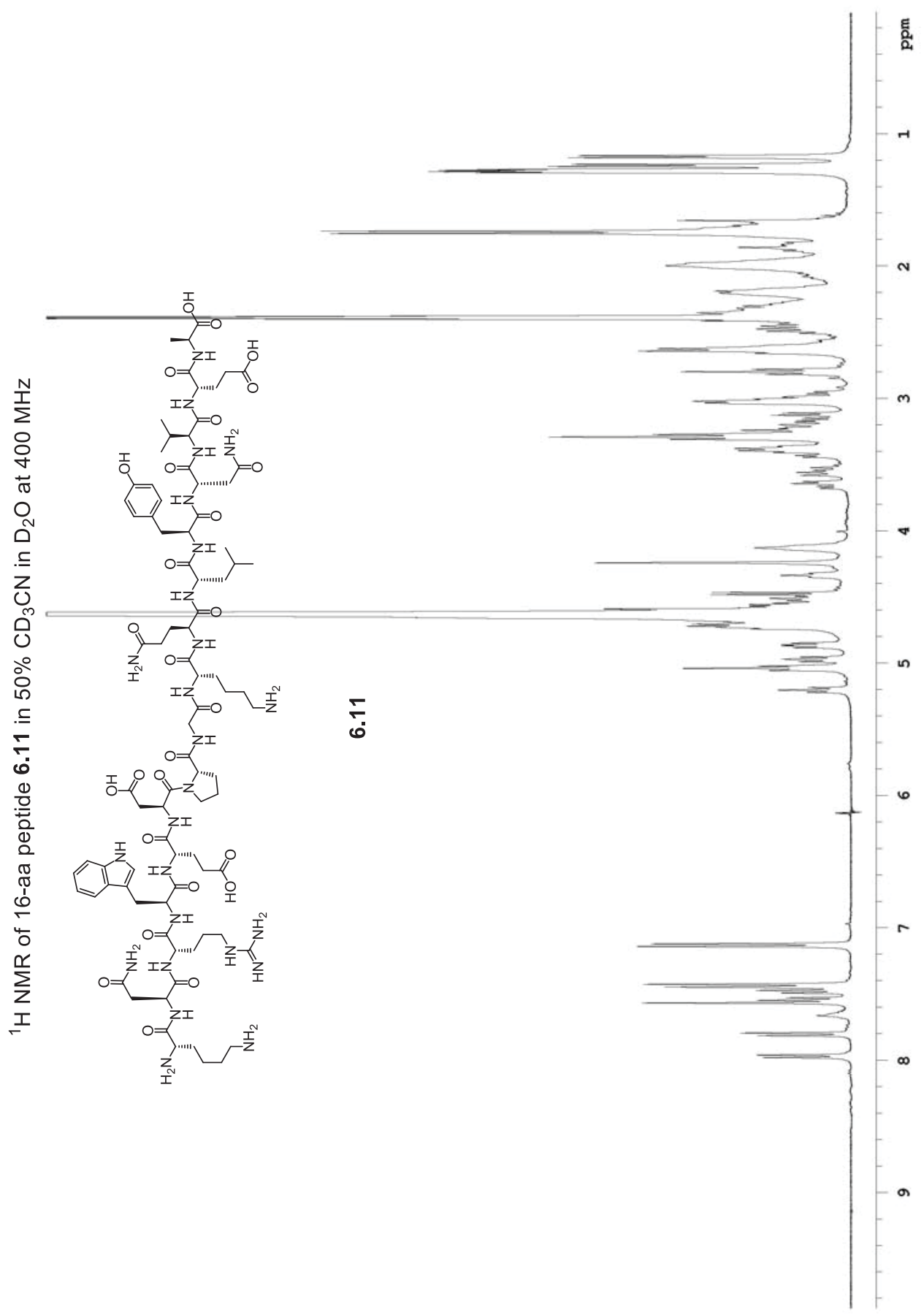

Figure D.16. ${ }^{1} \mathrm{H}$ NMR of 6 -aa peptide $\mathbf{6 . 1 1}$ 


\title{
Appendix E \\ Permission from Publisher for Chapter 2
}

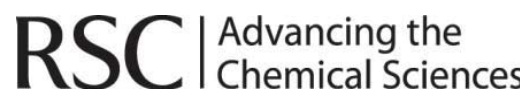

\author{
Royal Society of \\ Chemistry \\ Thomas Graham \\ House \\ Science Park \\ Milton Road \\ Cambridge \\ CB4 OWF
}

Tel: +44 (0)1223420 066

Fax: +44 (0)1223 423623

Email: contracts-

copyright@rsc.org

www.rsc.org

\section{Acknowledgements to be used by RSC authors}

Authors of RSC books and journal articles can reproduce material (for example a figure) from the RSC publication in a non-RSC publication, including theses, without formally requesting permission providing that the correct acknowledgement is given to the RSC publication. This permission extends to reproduction of large portions of text or the whole article or book chapter when being reproduced in a thesis.

The acknowledgement to be used depends on the RSC publication in which the material was published and the form of the acknowledgements is as follows:

- For material being reproduced from an article in New Journal of Chemistry the acknowledgement should be in the form:

o [Original citation] - Reproduced by permission of The Royal Society of Chemistry (RSC) on behalf of the Centre National de la Recherche Scientifique (CNRS) and the RSC

- For material being reproduced from an article Photochemical \& Photobiological Sciences the acknowledgement should be in the form:

o [Original citation] - Reproduced by permission of The Royal Society of Chemistry (RSC) on behalf of the European Society for Photobiology, the European Photochemistry Association, and RSC

- For material being reproduced from an article in Physical Chemistry Chemical Physics the acknowledgement should be in the form: 
o [Original citation] - Reproduced by permission of the PCCP Owner Societies

- For material reproduced from books and any other journal the acknowledgement should be in the form:

o [Original citation] - Reproduced by permission of The Royal Society of Chemistry

The acknowledgement should also include a hyperlink to the article on the RSC website. The form

of the acknowledgement is also specified in the RSC agreement/licence signed by the corresponding author.

Except in cases of republication in a thesis, this express permission does not cover the reproduction of large portions of text from the RSC publication or reproduction of the whole article or book chapter.

A publisher of a non-RSC publication can use this document as proof that permission is granted to use the material in the non-RSC publication.

VAT Registration Number: GB 342176471

Number: 207890

Registered Charity 


\section{Appendix F \\ Permission from Publisher for Chapter 3}

\section{BENTHAM OPEN}

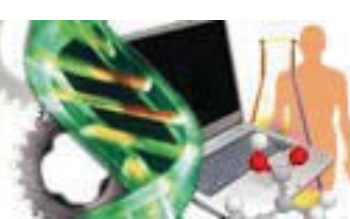

\section{Bentham OPEN - Policies}

1. All articles are freely available and immediately accessible online upon publication.

2. Readers can study, download and/or print $O P E N$ access articles at no cost.

3. Articles are licensed under the terms of the Creative Commons Attribution non- commercial License (http://creativecommons.org/licenses/by-nc/3.0/) which permits unrestricted, non-commercial use, distribution and reproduction in any medium, providing that the work is properly cited.

4. Bentham OPEN follows the single blind peer-review procedure for submissions of all manuscripts to its journals. Single blind is the most common type of peer- reviewing in which the identity of the reviewers is not disclosed to the authors of the submitted manuscript concerned. The anonymity of reviewers allows for objective assessment of the manuscript by reviewers and also free from any influence by the authors on the reviewers comments.

5. All submitted articles are subject to an extensive peer review in consultation with members of the Journal Editorial Board and independent external referees ; (usually three reviewers). All manuscripts are assessed rapidly and 
the decision based on all the peer reviewers comment, taken by the Journal Editor-in-Chief, is then conveyed to author(s).

6. All efforts are made to expedite the peer review process leading towards timely publication.

7. Authors publishing with Bentham OPEN retain the copyright to their work.

8. Authors have the flexibility to publish a wide range of articles in a Bentham OPEN journal e.g. short communications, full-length research and review articles, supplements, conference proceedings, and case studies.

9. Ethical Approval of Studies and Informed Consent: For human or animal experimental investigations, it is a prerequisite to provide a formal review and approval, or review and waiver, by an appropriate institutional review board or ethics committee and should be documented in your paper. For investigations undertaken on human subjects, the manner in which the informed consent was obtained from the study participants (i.e., oral or written) should be stated in the Methods section. Authors are encouraged to obtain patient consent when they use confidential case material. Consent is not necessary in the case of very brief case vignettes which do not contain identifying information or if the case material is disguised sufficiently to prevent identification of the patient. In obtaining consent, the author(s) should discuss the purpose(s) of publication, the possible risks and benefits to the patient and the patient's right to withhold or withdraw consent. In the case of a minor patient, consent should be obtained from the parent(s) or guardian(s) and assent should be obtained from the patient.

10. Standard Protocol on Approvals, Registrations, Patient Consents \& Animal Protection: All clinical investigations must be conducted according to the Declaration of Helsinki principles. Authors must comply with the guidelines of the international Committee of Medicinal Journal Editors (http://www.icmje.org) with regard to the patient's consent for research or participation in a study. Patient's names, initials or hospital numbers must not be mentioned anywhere in the manuscript (including figures). Editors may request that authors provide documentation of the formal review and recommendation from the institutional review board or ethics committee responsible for oversight of the study. 
11. Appeals and Complaints: Authors who wish to make a complaint should refer it to the Editor in Chief of the journal concerned. Complaints to the Publisher may be emailed to oa@ benthamscience.org

12. Plagiarism Prevention: Bentham Open uses the iThenticate software to detect instances of overlapping and similar text in submitted manuscripts. iThenticate software checks content against a database of periodicals, the Internet, and a comprehensive article database. It generates a similarity report, highlighting the percentage overlap between the uploaded article and the published material. Any instance of content overlap is further scrutinized for suspected plagiarism according to the publisher's Editorial Policies. Bentham OPEN allows an overall similarity of $20 \%$ for a manuscript to be considered for publication. The similarity percentage is further checked keeping following important points in view:

\section{Low Text Similarity:}

The text of every submitted manuscript is checked using the Content Tracking mode in iThenticate. The Content Tracking mode ensures that manuscripts with an overall low percentage similarity (but which may have a higher similarity from a single source) are not overlooked. The acceptable limit for similarity of text from a single source is $5 \%$. If the similarity level is above 5\%, the manuscript is returned to the author for paraphrasing the text and citing the original source of the copied material.

It is important to mention that the text taken from different sources with an overall low similarity percentage will be checked as a plagiarized content if the majority of the article is a combination of copied material.

\section{High Text Similarity:}

There may be some manuscripts with an overall low similarity percentage, but a higher percentage from a single source. A manuscript may have less than $20 \%$ overall similarity but there may be $15 \%$ similar text taken from a single article. The similarity index in such cases is higher than the approved limit for a single source. Authors are advised to thoroughly rephrase the similar text and properly cite the original source to avoid plagiarism and copyright violation.

\section{Types of Plagiarism:}

We all know that scholarly manuscripts are written after thorough review of 
previously published

articles. It is therefore not easy to draw a clear boundary between legitimate representation and plagiarism. However, the following important features can assist in identifying different kinds of plagiarized content. These are:

- Reproduction of others words, sentences, ideas or findings as one's own without proper acknowledgement.

- Text recycling, also known as self-plagiarism. It is an author's use of a previous publication in another paper without proper citation and acknowledgement of the original source.

- Paraphrasing poorly: Copying complete paragraphs and modifying a few words without changing the structure of original sentences or changing the sentence structure but not the words.

- Verbatim copying of text without putting quotation marks and not acknowledging the work of the original author.

- Properly citing a work but poorly paraphrasing the original text is considered as unintentional plagiarism. Similarly, manuscripts with language somewhere between paraphrasing and quoting are not acceptable. Authors should either paraphrase properly or quote and in both cases, cite the original source.

- Higher similarity in the abstract, introduction, materials and methods, and discussion and conclusion sections indicates that the manuscript may contain plagiarized text.

Authors can easily explain these parts of the manuscript in many ways. However, technical terms and sometimes standard procedures cannot be rephrased; therefore Editors must review these sections carefully before making a decision.

\section{Plagiarism in Published Manuscripts:}

Published manuscripts which are found to contain plagiarized text are retracted from the journal website after careful investigation and approval by the Editor-in- Chief of the journal. A 'Retraction Note' as well as a link to the original article is published on the electronic version of the plagiarized 
manuscript

13. Copyrights: Authors who publish in Bentham OPEN journals retain copyright to their work. Submission of a manuscript to the respective journals implies that all authors have read and agreed to the content of the Covering Letter or the Terms and Conditions. It is a condition of publication that manuscripts submitted to this journal have not been published and will not be simultaneously submitted or published elsewhere. Bentham OPEN (Licensor) grants the author(s) a worldwide, royalty-free, non- exclusive, and non-commercial perpetual license to exercise the rights in the article published as stated below:

a. All articles are published under the Creative Commons Attribution License

b. (http://creativecommons.org/licenses/by/3.0/) which permits unrestricted, non- commercial use, distribution and reproduction in any medium, providing that the work is properly cited.

b. The authors retain the copyright of their published article. They will also have the right to:

i. Reproduce the article, to incorporate the article into one or more collective

Works, and to reproduce the article as incorporated in the Collective Works;

ii. Create and reproduce Derivative Works for educational

purposes.

iii. Distribute copies

iv. Any commercial application of the work, with prior agreement by the author, is exclusively granted to Bentham OPEN

14. Waiver: Authors grant to the Bentham $O P E N$ (licensor) to retain all revenue from commercial sales of the author's published article in a Bentham $O P E N$ journal.

15. Bentham $O P E N$ offers affordable article processing fees, ranking amongst the lowest as compared to those of other OPEN access journal publishers. An 
article-processing fee payable by the author/ author's institution applies for every accepted article, to cover the costs incurred by OPEN access publication. Members of Bentham OPEN are entitled to discounted article processing fees.

16. Authors can self-archive post prints of their published articles.

17. Authors can reproduce derivative works of the article for educational purposes and distribute its copies. 


\section{Publication Charges Policy:}

Bentham OPEN is committed to disseminating research and scholarly publications as widely as possible. It supports the principle that the results of research that have been publicly funded should be freely accessible in the public domain' and therefore it encourages researchers to make their research available through Open Access (OA). Open access publishing is not without costs. To provide open access, Bentham OPEN journals partly defrays the expenses of peer review, journal production, and online hosting and archiving from authors and their research sponsors by charging a publication fee for each article they publish. The fees vary by journal. The publication fee details for each article published in Group ONE journals are given below:

Letters: The publication fee for each published Letter article submitted is USx $\$ 600$.

Research Articles: The publication fee for each published Research article is US \$800.

Mini-Review Articles: The publication fee for each published Mini Review article is US $\$ 600$.

Review Articles: The publication fee for each published Review article is US \$900.

The following special discounts are offered to authors and editorial board members:

- Authors from developing countries are entitled for $30 \%$ discount of their article publication fee submitted to any of the Group One journals.

- Authors from Bentham OPEN Member institutes are entitled for 30\% discount of their article publication fee submitted to any of the Group One journals.

- Editorial Board Members and Editors of Bentham OPEN journals are allowed ONE free publication annually. Subsequent submissions from Editorial Board Members are published at 50\% discount of the standard publication fee.

- Waiver and additional discount requests are decided on the basis of author's country of origin and quality of the submitted article. Editors and reviewers 
have no access to whether authors are able to pay; decisions to publish are only based on meeting the editorial criteria.

19. Errata and Corrections in Published Articles: Authors and readers are encouraged to notify the Editor-in-Chief if they discover errors in published content, authors' names and affiliations or if they have reasons for concern over the legitimacy of a publication. In such cases the journal will publish an ERRATUM in consultation with Editor-in-Chief and authors of the article, and/or replace or retract the article.

20. Article Withdrawal: Articles in Press (articles that have been accepted for publication or published as E-pub Ahead of Schedule but which have not been formally published with volume/issue/page information) that include errors, or are determined to violate the publishing ethics guidelines such as multiple submission, fake claims of authorship, plagiarism, fraudulent use of data or the like, may be "Withdrawn" from the journal. Withdrawal means that the article files are removed and replaced with a PDF stating that the article has been withdrawn from the journal in accordance with Bentham OPEN Editorial Policies.

21. Article Retraction: Published articles (with volume/issue/page information) which may contain infringements of professional ethical codes, such as multiple submission, bogus claims of authorship, plagiarism, fraudulent use of data or the like are retracted.

- A retraction note titled "Retraction: [article title]" signed by the authors and/or the

Editor-in-Chief is published in the paginated part of a subsequent issue of the journal and listed in the contents list.

- In the electronic version, a link is made to the original article.

- The online article is preceded by a screen containing the retraction note. It is to this screen that the link resolves; the reader can then proceed to the article itself.

- The original article is retained unchanged save for a watermark on the .pdf 
indicating on each page that it is "retracted."

- The HTML version of the document is removed.

22. Redundant (multiple) publication/ Re-publication: Abstracts and posters at conferences, results presented at meetings (for example, to inform investigators or participants about findings), results databases (data without interpretation, discussion, context or conclusions in the form of tables and text to describe data/information where this is not easily presented in tabular form) are not considered to be prior publication.

Authors who want to publish translations of the articles that has been published elsewhere should ensure that they have appropriate permission(s), should indicate clearly that the material has been translated and re-published, and should indicate clearly the original source of the material. The

Editor-in-Chief may request copies of related publications if they are concerned about overlap and possible redundancy opinions expressed therein, rests exclusively with the author(s) of such content. To the maximum extent permitted by applicable law, Bentham Open (on its own behalf, and on behalf of its staff and members of its editorial board) disclaims responsibility for any and all injury and/or damage (whether financial or otherwise) to persons or property, resulting directly or indirectly from any ideas, methods, instructions or products (including errors in the same) referred to in the content of any of Bentham OPEN journals. Any dispute arising, including any claim, shall be governed exclusively by the laws of the United Arab Emirates, as applied in the Emirate of Sharjah. 


\title{
Appendix G \\ Permission from Publisher for Chapter 6
}

\section{American Chemical Society's Policy on Theses and Dissertations}

\author{
If your university requires you to obtain permission, you must use the RightsLink \\ permission system. See RightsLink instructions at \\ http://pubs.acs.org/page/copyright/permissions.html.
}

This is regarding request for permission to include your paper(s) or portions of text from your paper(s) in your thesis. Permission is now automatically granted; please pay special attention to the implications paragraph below. The Copyright Subcommittee of the Joint Board/Council Committees on Publications approved the following:

Copyright permission for published and submitted material from theses and dissertations ACS extends blanket permission to students to include in their theses and dissertations their own articles, or portions thereof, that have been published in ACS journals or submitted to ACS journals for publication, provided that the ACS copyright credit line is noted on the appropriate page(s).

Publishing implications of electronic publication of theses and dissertation material Students and their mentors should be aware that posting of theses and dissertation material on the Web prior to submission of material from that thesis or dissertation to an ACS journal may affect publication in that journal. Whether Web posting is considered prior publication may be evaluated on a case-by-case basis by the journal's editor. If an ACS journal editor considers Web posting to be "prior publication", the paper will not be accepted for publication in that journal. If you intend to submit your unpublished paper to ACS for publication, check with the appropriate editor prior to posting your manuscript electronically.

Reuse/Republication of the Entire Work in Theses or Collections: Authors may reuse all or part of the Submitted, Accepted or Published Work in a thesis or dissertation that the author writes and is required to submit to satisfy the criteria of degree-granting institutions. Such reuse is permitted subject to the ACS' "Ethical Guidelines to Publication of Chemical Research" (http://pubs.acs.org/page/policy/ethics/index.html); the author should secure written confirmation (via letter or email) from the respective ACS journal editor(s) to avoid potential conflicts with journal prior publication*/embargo policies. Appropriate citation of the Published Work must be made. If the thesis or dissertation to be published is in electronic format, a direct link to the Published Work must also be included using the ACS Articles on Request author-directed link - see http://pubs.acs.org/page/policy/articlesonrequest/index.html

* Prior publication policies of ACS journals are posted on the ACS website at http://pubs.acs.org/page/policy/prior/index.html 
If your paper has not yet been published by ACS, please print the following credit line on the first page of your article: "Reproduced (or 'Reproduced in part') with permission from [JOURNAL NAME], in press (or 'submitted for publication'). Unpublished work copyright [CURRENT YEAR] American Chemical Society." Include appropriate information.

If your paper has already been published by ACS and you want to include the text or portions of the text in your thesis/dissertation, please print the ACS copyright credit line on the first page of your article: "Reproduced (or 'Reproduced in part') with permission from [FULL REFERENCE CITATION.] Copyright [YEAR] American Chemical Society." Include appropriate information.

Submission to a Dissertation Distributor: If you plan to submit your thesis to UMI or to another dissertation distributor, you should not include the unpublished ACS paper in your thesis if the thesis will be disseminated electronically, until ACS has published your paper. After publication of the paper by ACS, you may release the entire thesis (not the individual ACS article by itself) for electronic dissemination through the distributor; ACS's copyright credit line should be printed on the first page of the ACS paper.

\section{ACSPublications}

Most Trusted. Most Cited. Most Read.

Title:

Purification of Synthetic Peptides Using a Catching Full-Length Sequence by Polymerization Approach

Author: Mingcui Zhang, Durga Pokharel, Shiyue Fang

Publication: Organic Letters

Publisher: American Chemical Society

Date: $\quad$ Mar 1, 2014

Copyright (C) 2014, American Chemical Society
Logged in as:

Durga Pokharel

Account \#:

3000864555

\section{PERMISSION/LICENSE IS GRANTED FOR YOUR ORDER AT NO CHARGE}

This type of permission/license, instead of the standard Terms \& Conditions, is sent to you because no fee is being charged for your order. Please note the following:

- Permission is granted for your request in both print and electronic formats, and translations.

- If figures and/or tables were requested, they may be adapted or used in part.

- Please print this page for your records and send a copy of it to your publisher/graduate school. 
- Appropriate credit for the requested material should be given as follows: "Reprinted (adapted) with permission from (COMPLETE REFERENCE CITATION). Copyright (YEAR) American Chemical Society." Insert appropriate information in place of the capitalized words.

- One-time permission is granted only for the use specified in your request. No additional uses are granted (such as derivative works or other editions). For any other uses, please submit a new request.

\section{BACK}

CLOSE WINDOW

Copyright $\odot 2014$ Copyright Clearance Center, Inc. All Rights Reserved. Privacy statement. Comments? We would like to hear from you. E-mail us at customercare@copyright.com 